The copyright of this thesis vests in the author. No quotation from it or information derived from it is to be published without full acknowledgement of the source. The thesis is to be used for private study or noncommercial research purposes only.

Published by the University of Cape Town (UCT) in terms of the non-exclusive license granted to UCT by the author. 


\title{
Finite Element Analysis of Flows in Secondary Settling Tanks
}

\author{
by
}

\section{Dorothee Kleine}

Dipl-Ing. Rulu-Universil,ìi. Bochum, Germany (1992)

A lithsis submitted to the Department of Mathematics and Applied Wathernatics,

University of Cipe Town, in fulfilment of the muirements for the degree of

\section{Doctor of Philosophy}

at thes:

\section{UNIVERSITY OF CAPE TOWN}

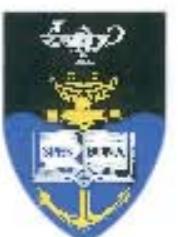

(c) University of Cape Tuwn

Seplember 2002 
To my parents 


\section{Declaration}

I doclare that this thesis is my own, unaided work. It is being submitted for the degree of Doctor of Philosophy in the University of Cape Town. It has not been subrittied before for any degree or examination in any other university.

Siguature of Author \begin{tabular}{c|c|} 
Signed by candidate \\
Signature removed
\end{tabular}

Department of Mathernatics and Applied Mathematics

Cape Town, September 2002 


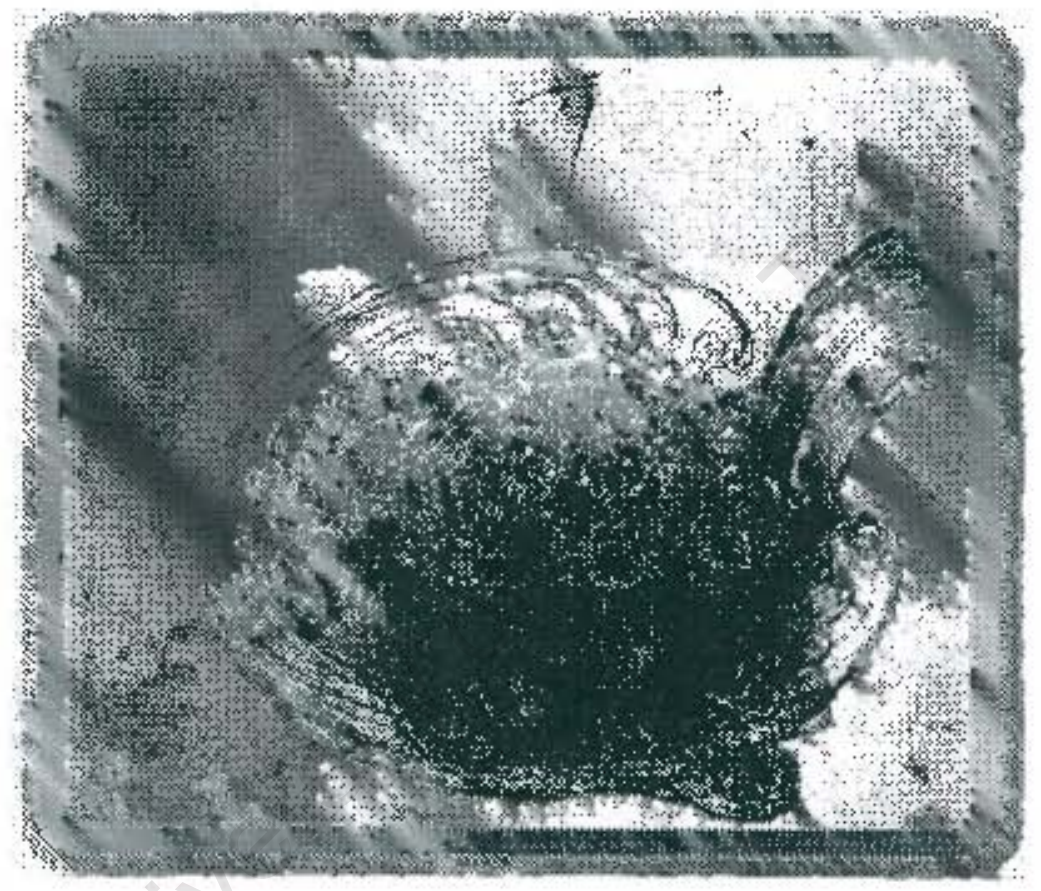




\section{Abstract}

Secondary settling tanks (SSTs) form a crucial part of wastewater treatment plants. Besides having to produce the separation of suspended solids and clarified effluent the secondary settling tank is used to concentrate and recycle the settled sludge to the biological reactor. The efficiency of the biological reactor in the waste water treatment system is determined by the efficiency of this final clarifying process. Hydrodynamic models have been developed for simulating secondary settling tanks in order to gain a better understanding of the complex flow patterns in these tanks, and to make design and optimization of the SST internal features possible. These models use mainly the finite volume method.

This thesis is concerned with the development and implementation of a finite element approach to the simulation of flows in SSTs. Although it is nowadays also possible to realise an unstructured grid within the FVM, the power of the finite element method (FEM) lies in its higher flexibility in fitting irregular domains and in providing local grid refinement. Generally, unstructured mesh procedures with the FVM require essential, additional orthogonality corrections, which affect the accuracy of the solution, and these corrections increase the computational cost due to the additional computations and increased iteration requirements. Structured mesh discretization may offer significantly shorter computation time. The FEM is therefore convenient for handling arbitrarily shaped domains and adaptation of complex internal features of SSTs, such as inlet and outlet arrangements.

The performance of a settling tank is determined by the tank hydraulics and the transport and removal of solids. The hydrodynamic SST model solves the continuity, momentum and the solids transport equations, as well as equations that model turbulence, namely the $k-\varepsilon$ model. A density state equation and a settling velocity equation carry out the coupling of the solids transport and 
In order to take into account these phenomena the two phases, water and suspended solids (SS), are treated as a homogeneous fluid mixture with variable density. The local density of this fluid mixture depends on the local concentration of the particles. A density-state equation then gives the local density of the fluid mixture with respect to the local solids concentration. The coupling between the momentum equation and the solids transport equation/density state equation is achieved through the gravity-density term in the momentum equation in the vertical direction

Generally, the variables are highly coupled, that is, the variable density results in an hydrostatic pressure, which strongly affects the velocities and vice versa; additionally, flow and settling motion react very sensitively to the values of turbulent viscosity and diffusivity.

Use is made of continuous equal-order interpolation of unknown variables on meshes of four-noded quadrilaterals. The solution of the coupled set of continuity and momentum equations is achieved through a time-splitting algorithm. The combination of two projection schemes, namely the projection schemes of Van Kan and of Chorin, is applied in order to unite the better accuracy and stability of Van Kan's second order pressure correction scheme in calculating the long-time behaviour of flows in SSTs, with the ability of Chorin's velocity correction scheme to eliminate the checkerboard pressure field, and in so doing to circumvent the need to satisfy the Babuska-Brezzi condition. The Fractional- $\theta$-scheme (FS) is adopted for time integration. The FS-scheme combines the advantages of both the backward Euler scheme and the Crank-Nicolson scheme. The entire system is decoupled through the use of an iterative scheme and linearization. The resulting symmetric and positive definite problem is solved by the conjugate gradient (CG) method, and the resulting non-symmetric problems of the discrete momentum, concentration and turbulence equations are solved by the generalized minimal residual (GMRES) method.

For the validation of the performance of SSTs the code is applied to full scale SSTs in two dimensions. The finite element analysis is tested against experimental data obtained from tests on real secondary settling tanks, and is compared with the computational results obtained with the 2D hydrodynamic SST simulation package SettlerCAD, which is based on the finite difference method. The finite element code shows reasonable results and is in good agreement with the experimental data, as well as with the computational results of SettlerCAD. 
Good results could also be obtained with the use of a constant turbulent viscosity, with the requirement that the inlet region is small compared to the settling section of the SST.

However, the computations show that (i) the simulation results react very sensitively to turbulent sizes; (ii) the laminar and turbulent diffusivity of the activated sludge mixture are of unknown size, and have to be adjusted in order to achieve the same results as those observed in the experiments; and (iii) mass balance cannot always be achieved due to the necessary and inevitably simplifications of $2 \mathrm{D}$ models. As a result of these uncertainties and the immense effect of turbulence on the settling process, the computational process does not provide a definite answer. To properly validate the model further information on experiments and tests are needed for better calibration. 


\section{Acknowledgements}

I would like most sincerely to thank my supervisors, Professor Daya Reddy and Professor George Ekama, for their support and guidance throughout the project period. Over the years I have benefited greatly from their expertise and from the help and advice they have given me.

For financial assistance I am grateful to the Water Research Commission (WRC), and to my supervisors.

I also wish to thank Dr. Richard Lord for his help and assistance regarding graphical postprocessing and operating systems.

Thanks are also due to Prof. Mark Wentzel and Dr. Howard Pearce for the fruitful discussions we have had on a variety of topics. 


\section{Contents}

Declaration ii

Abstract iii

Acknowledgements vi

Contents vi

List of Figures $\quad$ xi

List of Tables $\quad$ xvi

List of Symbols $\quad$ xvil

List of Abbreviations $\quad \mathrm{xx}$

1 Introduction $\mathbf{1}$

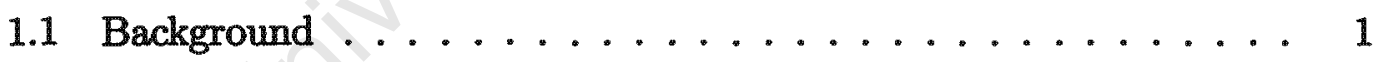

1.2 The use of the finite element method to simulate flows in SSTs . . 3

1.3 Thesis objectives .................... 5

1.4 Organisation of the thesis ................. 6

1.5 Contributions in this work ................. 8

2 Secondary settling tanks 10

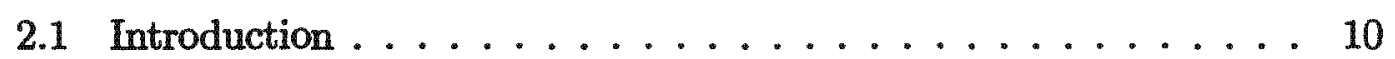

2.2 Functions of SSTs . . . . . . . . . . . . . . 10

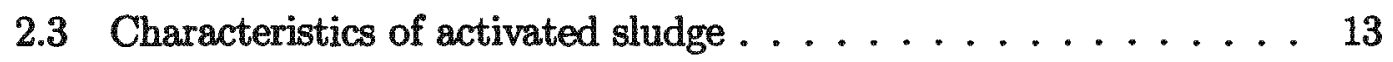

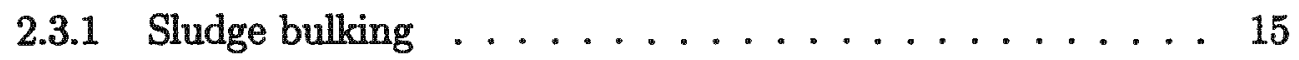

2.3.2 Flocculation and breakup ................ 15

2.3.3 Formation of floating solids ............ 16

2.4 Measurement of sludge settling and flocculation properties . . . 16 
2.4.1 Settling behaviour of biological sludges . . . . . . . 16

2.4 .2 Sludge volume indices ... . . . . . . . . . 19

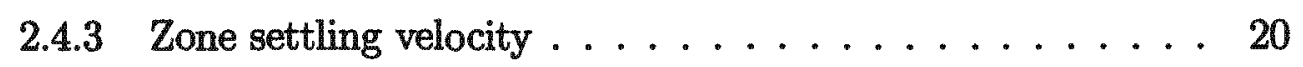

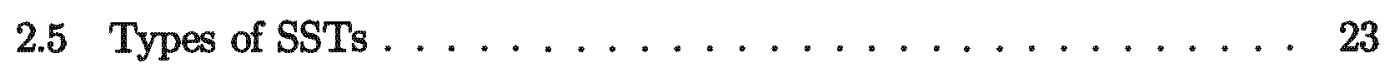

2.5.1 Rectangular secondary settling tanks ......... 24

2.5.2 Circular secondary settling tanks ........... 25

2.5.3 Vertical secondary settling tanks .......... 26

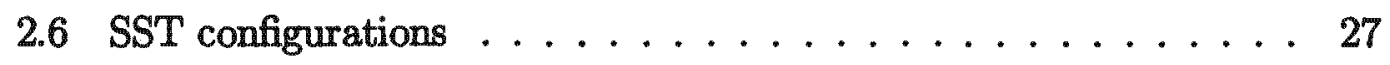

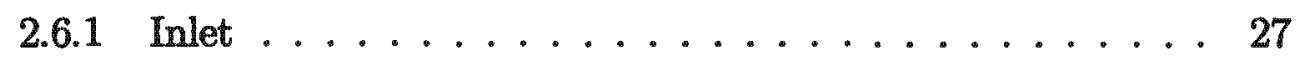

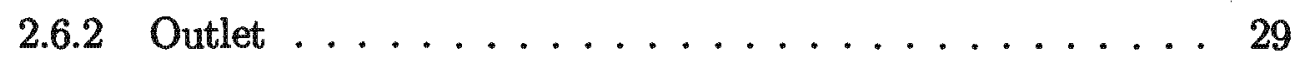

2.6.3 Sludge removal ................... 30

2.7 Closure . . . . . . . . . . . . . . . . . 31

3 Experiments and numerical modelling related to flows of SSTs 32

3.1 Experiments ...................... 32

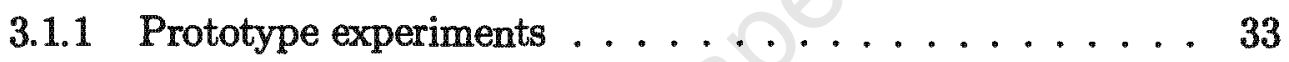

3.1 .2 Pilot scale tests . . . . . . . . . . . . 36

3.1.3 Laboratory scale experiments ............. . 37

3.2 Hydrodynamic modelling . . . . . . . . . . . . . . . 40

3.2.1 One-dimensional (1D) models . . . . . . . . . . . 41

3.2.2 Two-dimensional (2D) models: theoretical and computational research . ................ 42

3.3 Closing comment ...................... 48

4 Fundamental equations $\quad \mathbf{5 1}$

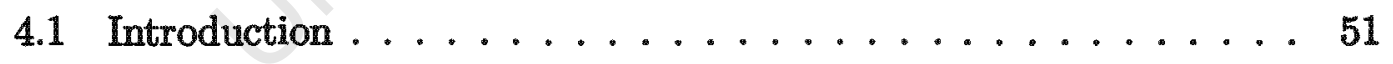

4.1 .1 Notation ................... 54

4.2 Conservation principles .................. 55

4.2 .1 Basic kinematics .................. 55

4.2.2 The Reynolds' transport theorem . . . . . . . . . 57

4.3 Conservation of mass: continuity equation .......... 58

4.4 Conservation of linear momentum: Navier-Stokes equations . . . 59

4.4.1 Cauchy's stress principle .............. 60

4.4.2 Conservation of linear momentum .......... 60

4.4.3 Incompressible Newtonian fluid ........... 61

4.4.4 The Navier-Stokes equations for incompressible flows . . 62

4.4.5 The Navier-Stokes equations for flows in SSTs ...... 62 
4.5 Conservation of particulate mass: the concentration equation . . 6 63

4.5.1 Solid settling equation . . . . . . . . . . . 64

4.5.2 Equation of state for density . . . . . . . . . . 65

4.6 Turbulence. . . . . . . . . . . . . . . . 67

4.6.1 The nature of turbulence . . . . . . . . . . . 67

4.6.2 Time-averaging procedure $\ldots \ldots \ldots \ldots \ldots 68$

4.6.3 The concept of eddy viscosity . . . . . . . . . . 69

4.6.4 The equation for turbulent energy $k \ldots \ldots \ldots \ldots 70$

4.6.5 The $k-\varepsilon$ turbulence model . . . . . . . . . . . 72

4.6.6 The universal $\log -\mathrm{law}$ of the wall $\ldots \ldots \ldots \ldots . . .73$

4.6.7 Wall functions . . . . . . . . . . . . . . 74

4.6.8 The $k-\varepsilon$ equations for SSTs $\ldots \ldots \ldots \ldots \ldots 76$

5 Numerical modelling $\quad 77$

5.1 Introduction . . . . . . . . . . . . 77

5.2 The method of weighted residuals $\ldots \ldots \ldots \ldots \ldots 78$

5.3 Spatial discretization and weak formulation . . . . . . . 80

5.4 The finite element method . . . . . . . . . . . . 85

5.4.1 Restrictions on the choice of velocity-pressure elements . . 89

5.4.2 Difficulties with the classical Galerkin method . . . . . . 91

5.5 Time discretization ................ . . 92

5.5.1 Single-step schemes . . . . . . . . . . . . . . 93 93

5.5 .2 Stability considerations . . . . . . . . . . 9 95

5.5.3 A fractional step scheme . . . . . . . . . . . . . 996

5.6 Derivation of the Navier-Stokes solver . . . . . . . . . . . . 97

5.6.1 Circumventing the Babuska-Brezzi restriction . . . . . . 99

5.7 Solution of systems of linear equations . . . . . . . . . 101

5.7.1 Gram-Schmidt orthogonalization . . . . . . . . . . 102

5.7.2 The Conjugate Gradient (CG) Method . . . . . . . . 103

5.7.3 The generalized minimal residual (GMRES) method. . . . 105

5.8 Solving the full set of equations . . . . . . . . . . 106

5.8 .1 Linearization . . . . . . . . . . . . . . . . . 106

5.8.2 An overall view of the algorithm . . . . . . . . . 107

5.8 .3 The program . . . . . . . . . . . . . 108

5.8 .4 Errors and tolerances . . . . . . . . . . . 111

6 Numerical evaluation $\quad 112$ 
6.1 Benchmark results for the neutral density case . . . . . . 113

6.1 .1 Lid-driven cavity . . . . . . . . . . . . . . . 113

6.1 .2 Backward-facing step . . . . . . . . . . . . . . 114

6.1.3 Flow between two parallel plates . . . . . . . 115

6.1 .4 Results . . . . . . . . . . . . . . . . 116

6.2 Simulation of flows in SSTs . . . . . . . . . . . 120

6.2.1 Geometry and boundary conditions . . . . . . . . . 121

6.2.2 Simulating the Darvill old and new SSTs . . . . . . 127

6.2.3 Simulating the Watts SSTs . . . . . . . . . . 129

6.3 Conclusion . . . . . . . . . . . . . . . . 132

7 Conclusions, and scope of future research 152

7.1 Opening remarks . . . . . . . . . . . . 152

7.2 Motivations and findings . . . . . . . . . . . 152

8 References 158 


\section{List of Figures}

2.1 The activated sludge system incorporating the secondary settling tank for solid/liquid separation. . . . . . . . . . . 11

2.2 Paragenesis diagram for particle settling behaviour for decreasing suspension concentration (vertical axis) and increasing tendency towards a particle flocculation (horizontal axis). . . . . . . . 14

2.3 Typical solids concentration-depth profile in SSTs. . . . . . . 14

2.4 Chronological progress of a stirred batch test showing the different settling regimes in the column at the different stages of settlement. 18

2.5 Plot of solid/liquid interphase depth against time in an unstirred measuring cylinder showing appearance of the different stages of settlement at the solid/liquid interface. . . . . . . . . . 18

2.6 Settling function of Takács et al. (1991) incorporating the settleability of both dispersed and flocculated suspended solids. . . . 22

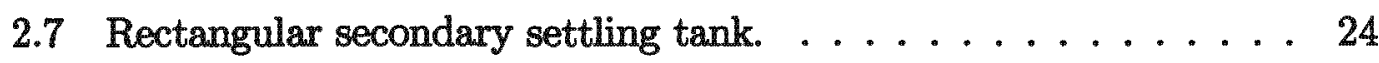

2.8 Centre feed, peripheral take-off, secondary settling tank. . . . . 25

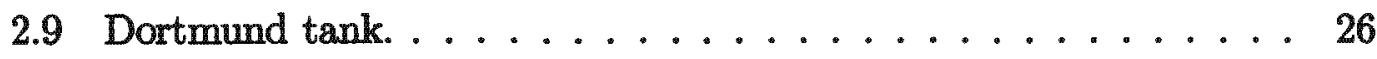

2.10 Typical features of a centre-feed circular SST. . . . . . . . 27

2.11 Arrangement of an inboard launder. . . . . . . . . . 29

2.12 Sludge removal systems for rectangular SSTs: (a) blade scraper system, (b) flight scraper system. . . . . . . . . . . 30 
3.1 Flow pattern and solids distribution in a circular secondary settling tank (Anderson 1945). The tail of each arrow indicates the start of a velocity reading. Velocities are shown in $\mathrm{mm} / \mathrm{s} . \ldots . .33$

3.2 Typical flow-through curves for tanks (Camp 1946). . . . . . . . 35

3.3 The three types of ideal reactors: batch reactor, plug flow reactor and mixed flow reactor. . . . . . . . . . . . 35

3.4 Typical secondary settling tank flow patterns found by Larsen (1997).

3.5 (a) Three layer-structure and (b) three layer-structure with shortcircuiting (Van Marle \& Kranenburg 1994). . . . . . . . . . . . 39

3.6 Numerical grid used for the computations; and comparison of streamline plot under two different model assumptions: (a) no effects due to density differences and (b) effects of density differences included. . . . . . . . . . . . . . . . 45

4.1 Modelling of fluid-solid flow in SSTs. . . . . . . . . . . 54

4.2 Coordinate systems for Cartesian and cylindrical coordinates. . . 55

4.3 Control volume in space and control volume of fixed mass moving with the flow. . . . . . . . . . . . . . . 55

4.4 Motion of an arbitrary volume in a continuum. . . . . . . 56

4.5 Finite control volume fixed in space. . . . . . . . 58

4.6 Arbitrary surface drawn in a fluid. . . . . . . . . . . . 60

4.7 Modified settling function of Takács. . . . . . . . . . . . 66

4.8 The state equation for density, $\ldots \ldots \ldots \ldots \ldots 6$

4.9 The energy cascade process. $\ldots \ldots \ldots \ldots \ldots$

4.10 Laminar and turbulent velocity distribution for the steady and unsteady case. . . . . . . . . . . . . . . 69

5.1 Problem domain $\Omega$ and boundary $\Gamma \ldots \ldots \ldots \ldots \ldots$ 
5.2 Typical velocity-pressure elements for continuous and discontinuous pressure. . . . . . . . . . . . 86

5.3 The reference element for the four-node $C^{0}$-linear isoparametric quadrilateral. . . . . . . . . . . . . . 87

5.4 The reference basis function $\hat{N}_{1}(\xi, \eta)$ for the four-node $C^{0}$-linear isoparametric quadrilateral. . . . . . . . . . . . 87

5.5 The quadratic-linear (a) and biquadratic-linear (b) elements satisfy the $\mathrm{BB}$ condition, while the bilinear velocity-linear pressure element (c) does not. . . . . . . . . . . . . . 90

5.6 The Gram-Schmidt process. . . . . . . . . . . . . 102

5.7 The Gram-Schmidt process for the case of two vectors. . . . . 103

5.8 Flow chart for finite element code NACOFEA. . . . . . 110

6.1 Geometry and boundary conditions for the lid-driven cavity flow problem. . . . . . . . . . . . . . . . . 113

6.2 Geometry and boundary conditions for the backward facing step flow. . . . . . . . . . . . . . . . . . . . . . 114

6.3 Geometry and boundary conditions for the flow between two parallel plates. . . . . . . . . . . . . . 116

6.4 Flow pattern of the lid-driven cavity flow problem for different Reynolds numbers: $R e=100,400$, and $1000 \ldots \ldots \ldots \ldots$

6.5 Computed results at Reynolds numbers $R e=100,400$ and 1000: (1) contours of the horizontal velocity $u / U$; (2) contours of the vertical velocity $v / U$; (3) pressure $p$ contours. . . . . . 117

6.6 Profile of the horizontal velocity $u / U$ along the line $x / H$ for Reynolds numbers $R e=100,400$ and $1000 \ldots \ldots \ldots \ldots \ldots$

6.7 Profile of the horizontal velocity $u / U$ along the vertical line $x / H$ for Reynolds numbers $R e=100,400$ and 1000 computed by Ghia et al. $(1982,1989) . \ldots \ldots \ldots \ldots$. . . . . . . . . . 118

6.8 Flow pattern at Reynolds numbers $R e=400$ and $800 \ldots \ldots \ldots 119$ 
6.9 Reattachment length $x / h$ as a function of Reynolds number. . . . 119

6.10 Flow pattern and pressure contours. . . . . . . . . . 120

6.11 Geometry and boundaries for Darvill old and new tank, as well for the Watts SST. . . . . . . . . . . . . . . . . 122

6.12 Boundary conditions for the simulation of SSTs. . . . . . . 123

6.13 Flux boundary condition for the sludge concentration at the free surface and at the bottom of the tank. . . . . . . . 125

6.14 Coarse mesh of Darvill new tank ( $43 \times 29$ ), and the flow pattern for the neutral density case, as well as for the solids loaded flow after 10 and $40 \mathrm{~min} . \ldots \ldots \ldots . \ldots . \ldots 134$

6.15 Unstructured mesh of Darvill new tank ( $20 \times 58+34 \times 29)$, and the flow pattern for the neutral density case, as well as for the solids loaded flow after 10 and $40 \mathrm{~min} . \ldots \ldots \ldots . \ldots 135$

6.16 Mesh of $85 \times 25$ elements for Darvill old tank (a) and of $85 \times 58$ elements for Darvill new tank (b), as well as the flow patterns for the neutral density case $(\mathrm{c}$ and $\mathrm{d}) . \ldots \ldots \ldots \ldots . \ldots . \ldots 136$

6.17 Darvill old tank: After $100 \mathrm{sec}$ to after $10 \mathrm{~min}$, predicted flow pattern and sludge distribution $c$ in $g / \ell \ldots \ldots \ldots \ldots$

6.18 Darvill old tank: After 15 min to after 6 hours, predicted flow pattern and sludge distribution $c$ in $g / \ell \ldots \ldots \ldots \ldots 138$

6.19 Darvill new tank with a $13.3 \%$ higher influent flow: After $100 \mathrm{sec}$ to after $10 \mathrm{~min}$, predicted flow pattern and sludge distribution $c$

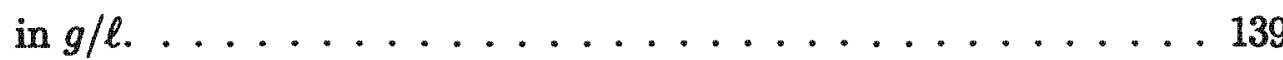

6.20 Darvill new tank with a $13.3 \%$ higher influent flow: After $10 \mathrm{~min}$ to after 6 hours, predicted flow pattern and sludge distribution $c$ in $g / \ell \ldots \ldots \ldots \ldots . \ldots . \ldots . . . \ldots 140$

6.21 Darvill old and new tanks: Steady state case, predicted flow pattern (a), horizontal velocity $u$ in $\mathrm{m} / \mathrm{s}$ (b,c), pressure field in $\mathrm{Pa}$ (d) and sludge distribution $c$ in $g / \ell(e) \ldots \ldots \ldots \ldots 141$ 
6.22 Watts tank: Mesh of $65 \times 30$ elements (a), predicted flow pattern for the neutral density case (b), predicted turbulent viscosity $\nu_{t}$ field for the neutral density case in $m^{2} / s$ (c). . . . . . . . 142

6.23 Watts test 1 with a Schmidt number of 1: predicted flow pattern (a), sludge distribution $c$ in $g / \ell(b)$ and predicted turbulent viscosity $\nu_{t}$ field in $m^{2} / s$ (c) . . . . . . . . . 143

6.24 Watts test 1 with a Schmidt number of 5: predicted flow pattern (a), sludge distribution $c$ in $g / \ell$ (b) and predicted turbulent viscosity $\nu_{t}$ field in $m^{2} / s$ (c) . . . . . . . . . 144

6.25 Watts test 12: After $100 \mathrm{sec}$ to after $10 \mathrm{~min}$, predicted flow pattern and sludge distribution $c$ in $g / \ell \ldots \ldots \ldots \ldots . \ldots 145$

6.26 Watts test 12: After $10 \mathrm{~min}$ to after 6 hours, predicted flow pattern and sludge distribution $c$ in $g / \ell \ldots \ldots \ldots \ldots \ldots$

6.27 Watts tests 4 and 12: Steady state case, predicted flow pattern (a), horizontal velocity $u$ in $\mathrm{m} / \mathrm{s}$ (b,c), pressure field in $\mathrm{Pa}$ (d) and sludge distribution $c$ in $g / \ell(\mathrm{e}) \ldots \ldots \ldots \ldots \ldots . \ldots 147$ 


\section{List of Tables}

2.1 Factors affecting the flocculation, settling,thickening and foaming characteristics of activated sludge (adapted from Ekama et al. 1997).................13

2.2 Factors affecting solid/liquid separation in SSTs, adapted from Ekama et al.

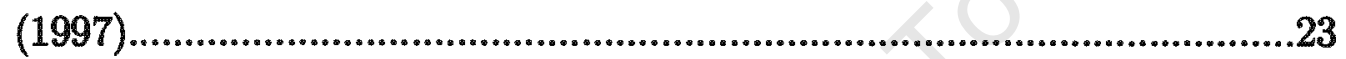

6.1 Summary of the loading conditions and model parameter used for the simulation of the Darvill old and new tanks...................................................148

6.2 Summary and comparison of computational and benchmark results for the simulation of Darvill old and new tanks. .149

6.3 Summary of the loading conditions and model parameter used for the simulation of the Watts tanks. 150

6.4 Summary and comparison of computational and benchmark results for the simulation of the Watts tanks. .151 


\section{List of Symbols}

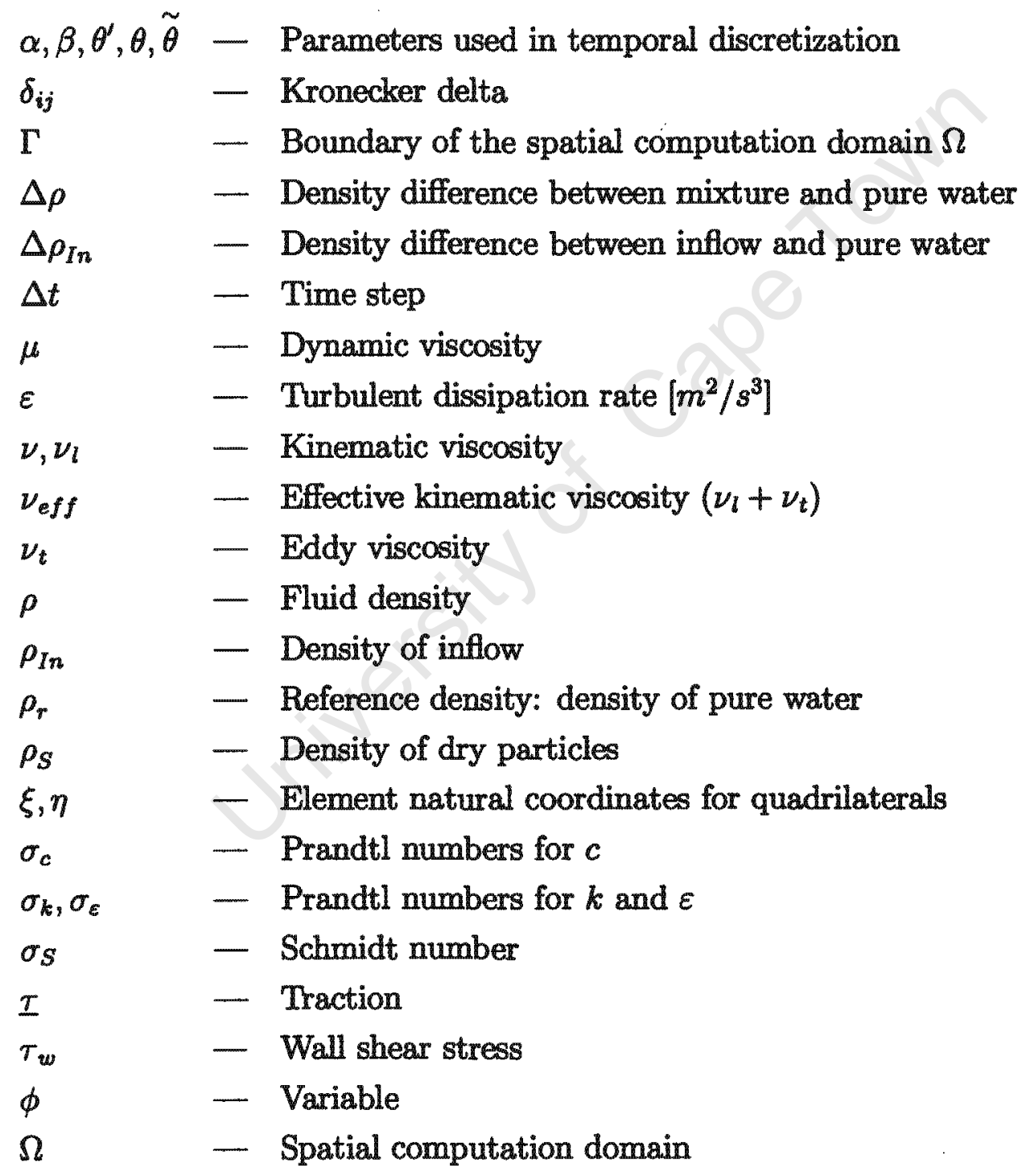




\begin{tabular}{|c|c|c|}
\hline$c$ & - & Suspended solids concentration \\
\hline$c_{\mu}, c_{1}, c_{2}, c_{\mu}^{\prime}, c_{D}$ & - & Constants used in $k-\varepsilon$ model \\
\hline $\mathrm{f}, \mathrm{F}, \underline{E}$ & - & Force vector \\
\hline$F_{D}$ & - & Densimetric Froude number \\
\hline$f_{n s}$ & - & Fraction of non-settleable particles \\
\hline$g$ & - & Acceleration due to gravity $\left(9.81 \mathrm{~m} / \mathrm{s}^{2}\right)$ \\
\hline$H$ & - & Total water depth in SST \\
\hline$H a$ & - & Hazen number \\
\hline$H_{I n}$ & - & Inlet aperture height \\
\hline $\mathbf{J}$ & - & Jacobian matrix \\
\hline$k$ & - & Turbulent kinetic energy $\left[\mathrm{m}^{2} / \mathrm{s}^{2}\right]$ \\
\hline$L$ & - & Length of SST \\
\hline$m$ & - & $\begin{array}{l}\text { Coordinate factor ( }=1 \text { for cylindrical, } \\
=0 \text { with } r=x \text { for Cartesian) }\end{array}$ \\
\hline$n$ & - & Time step number \\
\hline$n$ & - & Flux theory constant in semi-log $V_{S}-X$ relationship \\
\hline$\underline{n}, n_{i}$ & - & Outward unit normal vector \\
\hline$n_{2}, n_{4}$ & - & $\begin{array}{l}\text { Constants in double-exponential settleability } \\
\text { equation of Takács }\end{array}$ \\
\hline $\mathbf{N}$ & & Set of basis functions \\
\hline$N_{i}, M_{i}, L_{i}$ & - & Basis functions \\
\hline$\hat{N}_{i}$ & - & Local basis function of the reference element \\
\hline$N_{i}^{(e)}$ & - & Local basis function of the element \\
\hline$p$ & - & General pressure less the hydrostatic pressure $[\mathrm{Pa}]$ \\
\hline$p_{r}$ & - & Reference pressure \\
\hline $\mathrm{Pe}$ & - & Peclet number \\
\hline$r, y, \theta$ & - & Cylindrical coordinates \\
\hline $\operatorname{Re}$ & - & Reynolds number \\
\hline
\end{tabular}




$\begin{array}{ll}S & - \text { Surface } \\ S_{C} & - \text { Surface of the control volume } \\ t, T & - \text { Time } \\ T_{i j}, \underline{T} & - \text { Cauchy stress tensor, tensor } \\ u & - \text { Mean horizontal velocity in } r \text { - and } x-\text { directions } \\ U & - \text { Characteristic velocity } \\ u_{i} & - \text { Velocity tensor } \\ U_{I n} & - \text { Mean horizontal inlet velocity to the tank } \\ u_{i} & - \text { Intermediate velocity tensor } \\ u_{\tau} & - \text { Friction velocity } \\ u_{i}^{\prime}, p^{\prime}, C^{\prime} & - \text { Turbulent quantities of the instantaneous values } \\ \bar{u}_{i}, \bar{p}, \bar{c} & - \text { Mean quantities of the instantaneous values } \\ v & - \text { Mean vertical velocity in the } y-\text { direction } \\ V & - \text { Volume } \\ V_{c} & - \text { Control volume } \\ V_{m} & - \text { Material volume } \\ V_{S} & - \text { Settling velocity of sludge particles } \\ V_{S 0} & - \text { Flux theory constant in semi-log } V_{S}-X \text { relationship } \\ \mathbf{W} & - \text { Set of weighting functions } \\ W_{i}, M_{i}, L_{i} & - \text { Weighting functions } \\ x, y, z & - \text { Cartesian coordinates } \\ x & - \text { Horizontal coordinate } \\ x_{i}, \underline{x}, X_{i}, \underline{X} & - \text { Coordinate tensor } \\ y & - \text { Vertical coordinate } \\ S u p e r s c r i p t s & \text { and subscripts } \\ 0 & - \text { Initial conditions } \\ \mathrm{i}, j & - \text { Indices used in tensor notation } \\ & \end{array}$




\title{
List of Abbreviations
}

\author{
ATV - Abwassertechnische Vereinigung (Germany) \\ BB - Babuskka-Brezzi Condition \\ CG - Conjugate Gradient Method \\ DSVI - Diluted Sludge Volume Index \\ ESS - Effluent Suspended Solids \\ FDM - Finite Difference Method \\ FEM - Finite Element Method \\ FS - Fractional Step Scheme \\ FTC - Flow-Through Curve \\ FVM - Finite Volume Method \\ GMRS - Generalized Minimal Residual Method \\ HLR - Hydraulic Loading Rate \\ IAWQ - International Association on Water Quality \\ MLSS - Mixed Liquor Suspended Solids \\ ODE - Ordinary Differential Equation \\ PDE - Partial Differential Equation \\ PST - Primary Settling Tank \\ PWWF - Peak Wet Weather Flow \\ RAS - Return Activated Sludge \\ SLR - Solids Loading Rate \\ SS - Suspended Solids \\ SST - Secondary Settling Tank \\ SUPG - Streamline-Upwind Petrov-Galerkin Method \\ SVI - Sludge Volume Index \\ WRC - Water Research Commission (South Africa) \\ WWTP - Wastewater Treatment Plant \\ 1DFT - 1-Dimensional Flux Theory
}




\section{Chapter 1}

\section{Introduction}

\subsection{Background}

In water and wastewater treatment plants as well as in a variety of industrial processes, sedimentation tanks are used to separate suspended solids from water. Sedimentation by gravity is the most common and extensively applied treatment process for the removal of solids from water and wastewater.

The sedimentation basins of wastewater treatment plants are grit chamber, preliminary and secondary settling tanks, and the segregated materials are solids such as sand, grit or flocs generated in biological processes. Coming from the municipal sewer system, wastewater enters the wastewater treatment plant (WWTP) and passes first through screens and sieves in which large solids and debris are retained. Then the wastewater is pumped into primary settling tanks (PST), where a portion of the organic and inorganic solids settles out. The primary effuent is collected and redistributed to aeration tanks, which comprise the activated sludge process. Microorganisms biodegrade the organic material in the wastewater. Following aeration, the activated sludge (referred to as mixed liquor suspended solids, MLSS), is settled in final settling tanks, the so-called secondary settling tanks (SST), by gravity sedimentation. Part of the settled sludge is recycled back to the aeration tanks to maintain a high concentration of microorganisms in the tank. Excess sludge, created by the removal of organic pollutants and growth of microorganisms, is pumped to the sludge thickeners for further treatment. 
Clarifiers have a key role in the overall function of such waste water treatment plants. Besides having to produce the separation of activated sludge and clarified effluent, the secondary settling tank is used to concentrate and recycle the settled sludge to the biological reactor. The biological reactor tank and SST are strongly linked together and form a functional design and operational unit. Compared with industrial plants the SST for domestic sewage can be characterized by a high and unsteady load because of the occasionally added wet-weather flow. Furthermore, during and after transient load increase or reduction phase the deterioration of the effluent quality is mainly caused by sludge-blanket oscillations. The design and optimisation of clarifiers need for this reason to fulfil a complex set of design criteria, and for the simulation of the necessary design situations, including unsteady wet-weather flow, a numerical model is of great importance. The efficiency of the biological reactor in the wastewater treatment system can be determined through the efficiency of this final clarifying process. Furthermore, compliance with the effluent requirements depends strongly on the efficiency of the secondary settling tank.

Problems arise due to the two-phase nature of the flow in these tanks, which is in general unsteady due to variations of the inflow with time. Hydrodynamic and physical effects add to this complexity: examples are gravity sedimentation, density driven flow, flocculation, and thickening influence aspects of the tank performance such as flow patterns and the paths taken by the suspended solids (SS) through the settling tank.

The lack of knowledge regarding this complex flow often leads to false estimations with regard to the design of the inlet and outlet structures, and to oversizing during the renovation and construction of new plants. Since the investment in settling tanks, such as grit chamber, primary and secondary clarifiers usually amounts to approximately one third of the total investment costs, the determination of the efficiency of such tanks is of major importance and has been the subject of numerous experimental and theoretical studies.

One important aspect concerns the characteristics of the suspended solids (SS), that is, the distribution of the particle size and particle density, which along with the suspended solids concentration determine the settling velocity distribution of the SS. In addition, the characteristics of the SS can be affected by the flow field characteristics, resulting in particle agglomeration or break-up. The flow pattern determines the degree of turbulence in the tank, and with that the level 
of particle mixing. Turbulence keeps the particles in suspension and can even cause reentrainment of particles which have already settled. On the other hand, turbulence can also increase particle agglomeration.

Flow pattern, turbulence characteristics and sludge distribution are therefore of great interest to design engineers, and a proper determination of these features is essential for the prediction of tank efficiency.

Hydrodynamic models, that are based on the finite difference method (FDM) and on the finite volume method (FVM), respectively, have been developed for simulating secondary settling tanks in order to gain a better understanding of the complex flow patterns in these tanks, and to make design and optimization of the SST internal features possible.

The finite element method (FEM) belongs to the most important and most frequently used numerical methods. Originally, it was developed to solve strain problems within structural mechanics, but it has been extended very soon to the large application field of continuums mechanics. Structural mechanics works on the mechanical behaviour of discrete systems, which are described in general by ordinary differential equations. In continuum mechanics, such as elasticity and plasticity or fluid dynamics and heat transfer, field quantities/sizes are considered, that is, the displacement, velocities, temperature etc. are of interest in every point of the continuum. Mostly, these problems are nonlinear.

In the context of Hows in SSTs these nonlinearities are mainly to be found in the coupled nature of the problem, that is, water and sludge influence themselves mutually as a result of their different densities. Furthermore, the convective part in the conservation equations of mass and momentum, as well as the turbulent character of a flow are in general of physical nonlinearity. Engineers being confronted with these problems rely on numerical methods.

\subsection{The use of the finite element method to sim- ulate flows in SSTs}

The governing equations of the hydrodynamics of SSTs and the movement of sludge within these, to be described in Chapter 4, may be solved approximately by the finite element method (FEM). 
All numerical simulations are based on discrete approximations to the governing equations, and most of the differences in techniques arise from the choice of discretization of the problem. The most common approaches to the approximate solution of the PDE are the finite difference method (FDM), finite volume method (FVM) and finite element method (FEM).

The finite difference method approximates the derivatives in the differential equations via truncated Taylor series, and solves for discrete forms of the governing equations at specified nodes. The method is simple and efficient to code in one, two and three dimensions on structured grids, and it is easy to generate higherorder schemes. The restriction to simple geometries, static meshes, and problems with smoothly varying properties are significant disadvantages in complex flows.

The finite volume method is based on a discretization of the governing equations expressed in integral form. The solution domain is subdivided into a finite number of control volumes and the governing equations are applied to each control volume. The variable values are to be calculated at the centroid of each control volume and interpolation is used to express variable values at the control volume surface. Additionally, all terms that need to be approximated have physical meaning, which makes the FVM simple to understand and to program. A disadvantage of the FVM is the difficulty in defining the derivatives. Since the grid is not necessarily orthogonal, as in the FDM, the Taylor-expansion cannot be applied to define the derivatives. It is also not possible to convert higher order derivatives into lower ones with the mechanism of a weak formulation, as in the finite element method. The FVM is suitable for complex geometries, however, unstructured mesh procedures require essential, additional orthogonality corrections, which affect the accuracy of the solution and these corrections increase the computational cost due to the additional computations and increased iteration requirements, Structured mesh discretization may here offer significantly shorter computation time.

A weak formulation is the main distinguishing feature of the finite element method. The equations are multiplied by a weighting function before they are integrated over the entire domain. The calculation domain is subdivided into a set of discrete volumes or finite elements that are generally unstructured. Though it is nowadays also possible to realise an unstructured grid with the FVM, the power of the finite element method lies in its higher flexibility in fitting irregular domains and in providing local grid refinement. Furthermore, the FEM is able 
to accommodate problems with strongly varying internal properties, and can be applied to Lagrangian moving-mesh problems. The disadvantage of the FEM is its significantly greater computational complexity.

\subsection{Thesis objectives}

The purpose of this thesis is to develop and implement a finite element analysis code for simulating fluid and solids movements on full scale circular and rectangular SSTs. The flexible geometry of the FEM supports the description and adaptation of complex internal features of SSTs, such as inlet and outlet arrangements, as well as an easy conversion from finer to coarser grid by using different element types.

The hydrodynamic SST model solves the continuity, momentum (Navier-Stokes) and the solids transport equations, as well as the $k-\varepsilon$-turbulence equations, and the governing equations are those given in the work 'Secondary Settling Tanks: Theory, Modelling, Design and Operation', IAWQ STR, Ekama et al. (1997).

The treatment of the convection terms and the gravity-density term in the Navier-Stokes equations is an important issue to be addressed. The flow pattern in SSTs strongly depends on the viscosity and on buoyancy forces. Numerical instability additionally appears due to the increasing influence of the buoyancy source term.

Efficient and robust solution schemes are required for the coupled system of nonlinear equations. The characteristics of the complex and highly unstable flow in SSTs impose great challenges on the numerical and computational approaches. The problems that arise in the numerical treatment of these partial differential equations include the following:

- time-dependent partial differential equations in complex domains

- Problem: extremely high storage and computation expenditures

- strongly nonlinear systems of equations

- Problem: variable density, turbulence, iterative solver 
- incompressibility

- Problem: element choices, solution schemes

- local changes of the problem character in space and time

- Problem: stabilization of the convective terms, locally varying steps in time and space

- temporarily stiff systems of differential equations

- Problem: implicit time stepping schemes

The main goal of this work is to compile a working finite element package for 2D hydrodynamic modelling of SSTs that is capable of simulating the benchmark results available in the literature. The thesis objective should propose developments and advances in the computational problem areas outlined above.

Results are given for examples based on settling tank geometries and loading conditions. These results show graphically the flow patterns and the contours of sludge concentration. The degree of flow and solids maldistribution can be qualified and suggestions can be made concerning improvements.

From the research point of view it is of interest to compare the different computational approaches with regard to their advantages and disadvantages, and their performance. A very important issue is to know how well a FEM program can describe these highly unstable flows in SSTs. The FEM program will be evaluated against experimental data obtained from measurements on real SSTs, and will be compared to the SST specific FDM program SettlerCAD (Zhou et al. 1998).

\subsection{Organisation of the thesis}

Chapter 2 of the thesis gives an introduction to secondary settling tanks of waste water treatment plants based on activated sludge. It points out the difficulties that arise due to the complex characteristics of activated sludge, which combines flocculation properties, settling- and thickening behaviour, as well as the 
tendency to floating and foaming. Many factors affect the characteristics of activated sludge, such as wastewater composition and the biological system itself, and this complexity leads to the problematic nature of the design process. The crucial parameter is the settling behaviour, and two measurements have been developed that enable the quantitative description of this parameter, namely the sludge volume index and the zone settling velocity. They form the basis for the design of SSTs and are explained in more detail. Finally, the different types of SSTs and features such as inlet, outlet, and sludge removal, are briefly described.

After pointing out the difficulties owing to the complex nature of activated sludge and flows in SSTs, a review is given in Chapter 3 of experimental and numerical work on flows of SSTs. Experiments on SSTs, and the difficulties arising due to the complex flows in these tanks, are presented and outlined. The generally expensive and time-consuming experiments provide some of the motivation for the development of different mathematical models for flows in SSTs. Chapter 3 illustrates and outlines the problems and difficulties that arise due to the complex flows in SSTs and the numerical instabilities that occur with it.

Chapter 4 gives an introduction to the physics of liquid-solid flow and the different approaches to modelling such flows. The model used in this work is described, the fundamental equations are presented. These include the equations of conservation of mass, momentum and solid concentration. In Section 4.6 special attention is paid on the phenomenon of turbulence. The nature of turbulence is described with special reference to its importance and its immense influence on fluid flow and sludge concentration, and the concepts used to describe the physical manifestations of turbulence are discussed. Based on the time-averaging procedure and the eddy-viscosity concept the equation of the kinetic turbulent energy $k$ is derived. Due to the shortcomings of the single-equation turbulence models, the dissipation $\varepsilon$ of the kinetic turbulent energy $k$ as second variable and its conservation equation are presented. Finally, attention is paid to turbulent flows near solid walls, which finds its interpretation in the the universal log-law of the wall.

Chapter 5 gives a detailed description of the numerical model, starting with a description of the finite element method. In particular, the method of weighted residuals is presented, and the spatial discretization of the governing equations is described. These fundamental procedures give the background to the finite element method, which is then described in greater detail. The difficulties that 
arise with the use of the classical Galerkin method for incompressible flows are summarized and described. Time discretization is treated next, by firstly describing the most commonly used single-step- $\theta$-schemes, which then leads to the presentation of the fractional-step- $\theta$ scheme that is implemented in the present FEM code. In order to solve the coupled system of equations, a combination of two time-split projection schemes is applied. The reasons for combining two different projection schemes, namely the algorithm of Van Kan and of Chorin, are discussed. For the solution of the linear equation systems of the implemented nonstationary iterative methods, the CG and GMRES, are briefly described. In the last section of Chapter 5 the equation solution technique and the structure of the complete finite element program are explained in detail.

The performance of the algorithms and code are investigated in the first part of Chapter 6. Three representative benchmark tests, which are well documented in the literature, are given for the neutral density case. Then in Section 6.2 the results of simulations of SST flows are presented, and compared with experimental data obtained from measurements on real secondary settling tanks. Additionally, the computational results are compared with the simulation results of the 2D hydrodynamic SST simulation package SettlerCAD, which is based on the finite difference method.

Finally, in Chapter 7 conclusions are drawn on the work and results presented in this thesis, and recommendations for future research are made.

\subsection{Contributions in this work}

The candidate's original contributions in this thesis are summarised as follows:

This thesis is a contribution to the highly nonlinear and unstable solution procedure of density-driven flows, which occur for example in secondary settling tanks. The candidate's own code shows how efficiently these sensitive and unstable flows can be simulated by the finite element method (FEM) that has additionally the very important feature of being very flexible in handling complex geometry. The developed code is based on the fundamental equations described in the literature and mainly used by other researchers to simulate the flow of SSTs. With this code the extremely fragile flow behaviour of density-affected flow can be resolved. The code shows stability also for coarser grids. In order to take into account the 
different nature of water and sludge, such as viscosity, other physical models can easily be implemented into the code; however, they depend in the same way on further information on experiments and tests, and will have the same deficiencies as the model used in this thesis.

The development, coding, simulation and the implementation of the finite element method based on data of full-scale secondary settling tanks are the candidate's own work. This code is not based on any other work, and no package or other code has been used. 


\section{Chapter 2}

\section{Secondary settling tanks}

\subsection{Introduction}

This chapter provides an introduction to secondary settling tanks (SSTs) of the kind used in wastewater treatment plants that are based on activated sludge. The theory of settling and flocculation of activated sludge is described, as is the determination of sludge settling and flocculation properties. In addition, aspects of the design and performance of secondary settling tanks based on flocculation and sludge settleability are described. The overview draws on the work of (Ekama et al. 1997).

\subsection{Functions of SSTs}

In the secondary settling tank the activated sludge is separated from the treated wastewater by gravity sedimentation, and is returned to the process. The function of the SST is twofold: firstly, to separate the treated wastewater from the activated sludge mass, thereby producing a clear final effluent and secondly, to produce a continuous underflow of thickened sludge for return to the biological process. Figure 2.1 illustrates the simplified scheme of the activated sludge system. If the SST fails, suspended solids will escape with the effluent, resulting in an effluent that does not fulfil the effluent quality standards, and the receiving water will be polluted. Furthermore, should the loss of mixed liquor suspended solids (MLSS) be excessive (several $100 \mathrm{mg} / \ell$ ), biodegradation processes 


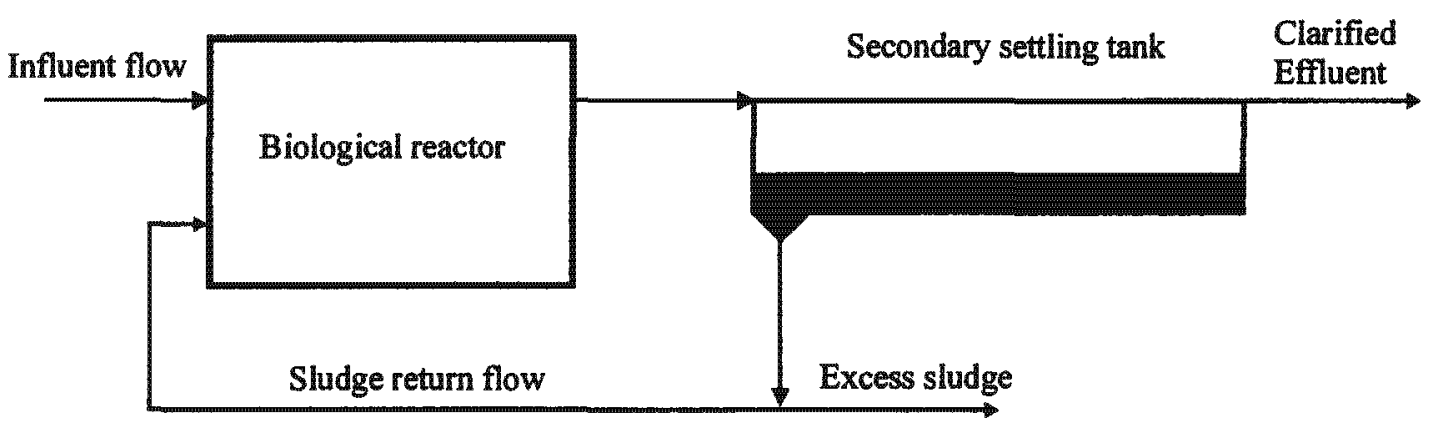

Figure 2.1: The activated sludge system incorporating the secondary settling tank for solid/liquid separation.

with high sludge ages, such as nitrification and denitrification, will cease. Consequently, biological reactor and SST are strongly linked together and form a functional unit in the design and operation of waste water treatment plants.

The SST combines the functions of thickening, clarification, and storage. As a thickener it produces a contimuous sludge underflow of thickened sludge for the return to the biological reactor; as a clarifier it separates suspended solids and water, thereby producing a clarified final effluent; and as a storage tank the SST stores sludge during wet weather peak flows. In the following section the functions of a SST are described in greater detail.

\section{The function of the SST as a thickener}

The thickening function of SSTs is defined by the settleability ${ }^{1}$ of the activated sludge. The settleability determines the reactor concentration and limits the treatment capacity of the activated sludge reactor-SST system. Different measures for sludge settleability have been developed and incorporated into theories and design procedures for SSTs, allowing for a design that optimizes the balance between sludge settleability and reactor concentration. In general, if the sludge settleability is worse than that designed for, the SST will limit the actual treatment capacity to less than the design value.

\section{The function of the SST as a clarifier}

Besides its biological treatment capacity the efficiency of the activated sludge system-SST system depends strongly on effective removal of particulate sus-

\footnotetext{
${ }^{1}$ The settleability of the activated sludge combines flocculation properties, settling- and thickening behaviour, as well as the tendency to floating and foaming.
} 
pended solids. High sludge concentration in the effluent can be a major contributor of organics/pollutants in activated sludge-SST system effluents. Reasons for excessive solids in the SST effluent include the following:

- High velocities, resulting in short-circuit flow or resuspension of solids from the surface of the sludge blanket

- Thickening overloads, resulting in a high sludge blanket, and huge loss of solids when the blanket reaches the effluent weir

- Denitrification, causing solids to float to the surface

- Poor flocculation, resulting in floc breakup or poor floc formation before settlement

- Insufficient transport and removal capacity of the sludge collection system, resulting in raised sludge blankets

\section{The function of the SST as a sludge storage zone}

During peak wet weather flows (PWWFs) solids are transferred from the aeration tank to the SST and retained there as a result of a temporary overload of the thickening function of the SST, as well as the requirement of an increased underflow concentration. The solids inventory transfer has an impact on the clarification function of the SST and due to the relatively low frequency of such peak events, very little is known about them. However, PWWFs are the most critical design condition for a SST. The allocation of extra volume within the SST during design is one way in which to absorb and store the solids inventory shift during wet weather conditions. Besides the increase of side water depth, a low solid loading rate (SLR) may ensure that the sludge blanket will not rise to the effluent outlet at PWWFs, and will preserve the effluent quality even in shallow tanks. On the other hand, this method requires either larger aeration tanks or step feed capability to maintain low mixed liquor feed concentrations to the SST during PWWFs.

In this context it is worth mentioning that with the use of CFD simulations the dynamic behaviour of a SST during peak events can be studied, and statements can be made about its storage function. 


\begin{tabular}{ll}
\hline Wastewater composition & Industrial contribution, soluble organic \\
& content, nutrients, temperature, $\mathrm{pH}$, to- \\
& tal dissolved solids (TDS), septicity, com- \\
& bined or separate sewers, oil and grease \\
& content. \\
\hline Biological system & Configuration, temperature, mixing, pH, \\
& aeration, presence of anoxic and/or anaer- \\
& obic zones, sludge age, reactor mixed \\
& liquor suspended solids (MLSS), dissolved \\
& oxygen (DO) concentrations. \\
\hline
\end{tabular}

Table 2.1: Factors affecting the flocculation, settling, thickening and foaming characteristics of activated sludge, adapted from Ekama et al. (1997).

\subsection{Characteristics of activated sludge}

Activated sludge is characterised through a combination of flocculation properties, settling-and thickening behaviour, as well as the tendency to floating and foaming. In general, the characteristics of activated sludge are affected by the composition of the influent wastewater and the conditions in the biological reactor. Table 2.1, adapted from (Ekama et al. 1997), summarises the relevant factors affecting the behaviour of activated sludge.

Solids settle in four different ways, depending on concentration and their flocculation properties. These settling types can be classified as follows:

- Class I: Discrete settling non-cohesive particles (e.g. sand)

- Class II: Discrete settling with flocculation (e.g. activated sludge at certain concentrations)

- Class III: Hindered settling, in which the particle mass settles as a unit and a sludge blanket develops

- Class IV: Thickening, in which compression takes place.

Figure 2.2 shows the partitioning of these sedimentation processes as a function of particle concentration and flocculation behaviour. Different regions in a SST can be assigned to the different classes: class I in the upper tank region, classes 


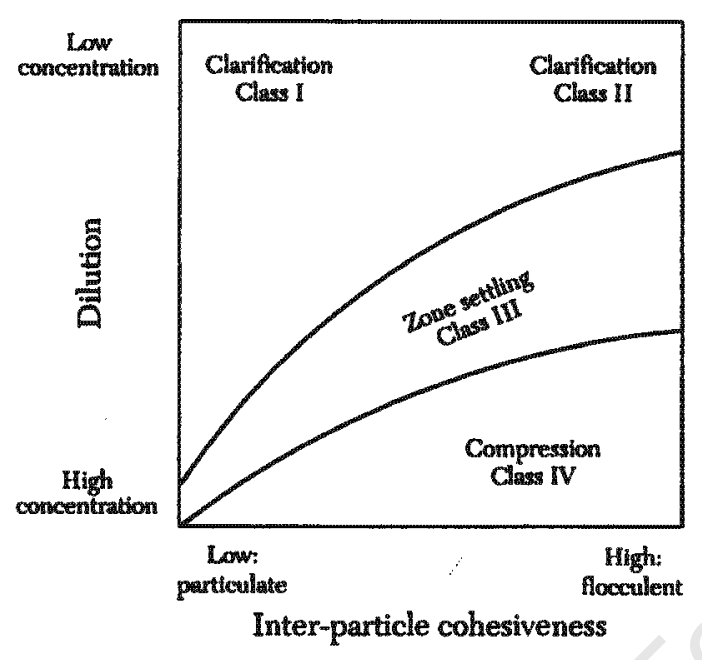

Figure 2.2: Paragenesis diagram for particle settling behaviour for decreasing suspension concentration (vertical axis) and increasing tendency towards a particle flocculation (horizontal axis).

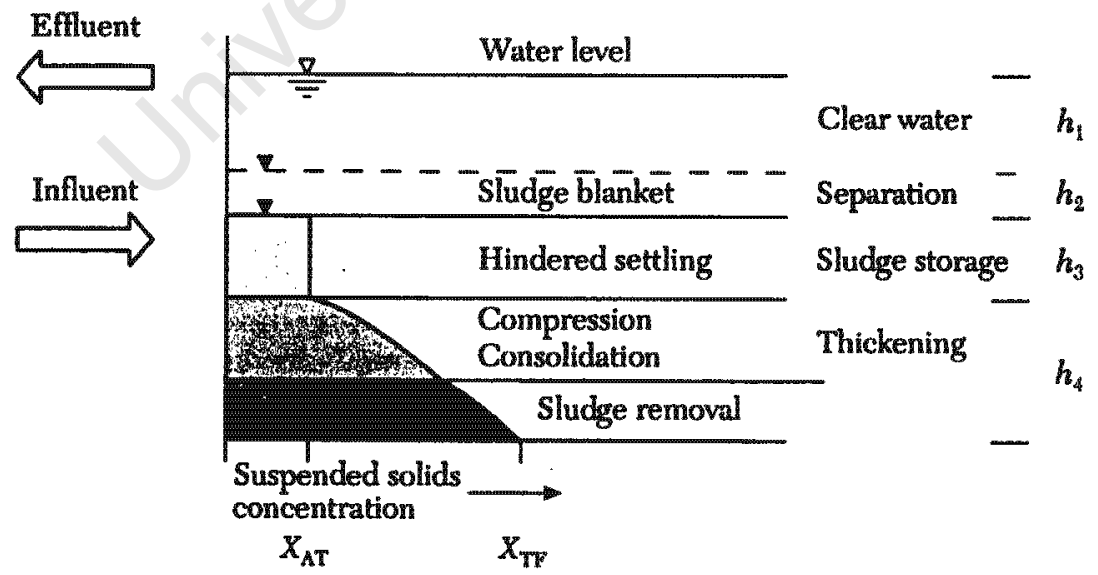

Figure 2.3: Typical solids concentration-depth profile in SSTs. 
II and III in the middle zone, and class IV at the bottom of the tank (see also Figure 2.3).

\subsubsection{Sludge bulking}

Some micro-organisms take the form of long filaments. The filamentous organisms can cause sludge bulking, which has a dramatic effect on the sludge settling characteristics. In nitrogen and nutrient (nitrogen and phosphorus) removal plants, the filamentous organism groups are associated with low food/microorganism ratios, and are the major cause of bulking problems. The sludge age is large in plants with low food/micro-organism ratios, and the wide range of possible operating conditions and configurations makes it difficult to determine the reasons for proliferation of these filaments. Today, after nearly a half century of research, sludge settleability control through control of filamentous organism growth remains probably the greatest unsolved problem of activated sludge treatment.

\subsubsection{Flocculation and breakup}

Good flocculation properties are vital for the performance of the activated sludge and solid contact processes, because they provide for the incorporation of slow, nonsettleable and small particles into larger floc particles with high settling velocity.

Two important conditions must be fulfilled in order to achieve sufficient flocculation: (i) good flocculation properties, which is in general the case for activated sludge, and (ii) relative movement of the sludge particles. Better contact between the solids can be achieved with sufficiently high shear gradients and a turbulent flow field. On the other hand, external influences, such as inflow velocities and jets, result in sharper velocity gradients and the breakup of flocs is likely to take place. For improved sludge flocculation, specific attention should be paid to create better conditions for the support of flocculation through, for example, the provision of better flocculation conditions in the inlet chamber of the SST. 


\subsubsection{Formation of floating solids}

Foam and scum formation in aeration tanks can be of a chemical, physical or biological nature, with the latter generally being dominant and very difficult to control. Long sludge ages and warm temperatures can stimulate relatively slow growing foaming organisms, such as Nocardia and Microthrix parvicella, to proliferate in the process.

Denitrification in SSTs is another factor that causes foam formation. Bubbles of nitrogen generated by denitrification in the SST cause solids to rise to the water surface. Additionally, stagnation regions with long duration of stay (dead zones) in the hopper region, for example, can induce denitrification. The minimization of denitrification requires a certain minimum capacity for sludge transport and collection in the final clarifier.

\subsection{Measurement of sludge settling and floccu- lation properties}

Two approaches have been developed to measure parameters that enable the quantitative description of settling characteristics. These are based on either the volume of sludge obtained after a fixed time of settlement, or the subsidence velocity of the solid/liquid interface. Both approaches use the batch settling column method, which forms the basis for different kinds of tests, as described below.

\subsubsection{Settling behaviour of biological sludges}

Due to its generally good flocculation properties even at low concentrations $(\approx 1000 \mathrm{gSS} / \ell)$, activated sludge shows zone settling behaviour (class III) in batch settling tests; that is, the particles of different sizes settle at the same rate throughout the zone depth. Four different stages of settling can be observed in a batch cylinder settling test: the lag (1), zone settling (2), transition (3), and compression (4) stages (see Figures 2.4 and 2.5).

- Lag Stage (1): A distinct interface between mixed liquor and supernatant 
(A) develops during this stage, which is of one to two minutes in duration from the start of the test. At the same time the mixed liquor (B) begins to move to the bottom, and transition and compression zones form at the bottom.

- Zone Settling Stage (2): In the zone settling stage (2) the mixed liquor (B) settles at a uniform velocity and constant concentration. In this region the gravity force and the friction force acting on the particles are in equilibrium. During the zone settling stage the transition zone (C) stays constant and transfers sludge particles from the decreasing zone settling layer (B) to the increasing compression layer (D) until the transition zone appears at the liquid/solid interface.

- Transition Stage (3): During this stage sludge particles are continuously transported to the compression layer, while the concentration in the transition layer increases with increasing depth. The transition layer contracts and ceases when the last layer reaches the compression stage.

- Compression Stage (4): When the compression stage is reached, the compression layer decreases in depth. The subsidence of the interface is now governed by the compression behaviour of the sludge. The compression stage is difficult to identify due to the variation in depth during the test. In addition, very little is known about the compression behaviour, and only empirical approaches can provide information about the compression rate (Merkel 1971, Pflanz 1969).

In general all four classes of settling take place in SSTs (Figure 2.3). Classes I and II characterise the effluent suspended solids (ESS), class III the solids transport to the bottom of the SST, and class IV the compaction of sludge on the SST floor. In the design of SSTs, the class III zone settling stage is assumed to dominate sludge settling behaviour and to govern the required surface area. While this is not an unreasonable assumption, it is not clear whether it is a convenient one, because zone settling is the only class for which the solids zone settling velocity versus concentration relationship can be measured. Classes I and II settling, which dominate water treatment plant sedimentation tanks, are not modelled on a solid settling velocity versus concentration basis, but on $\%$ solids removal versus overflow rate, and on an overflow rate and depth basis, 


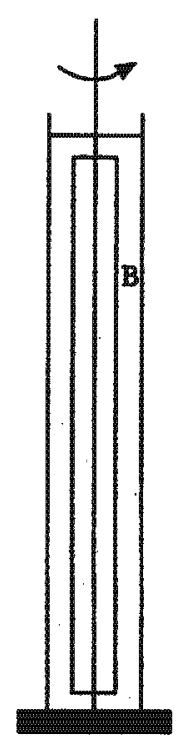

(a)

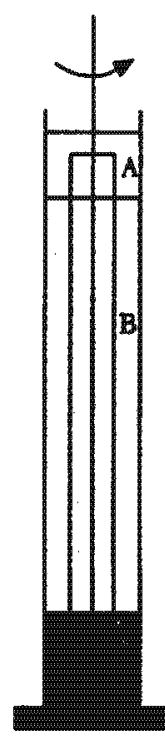

(b)

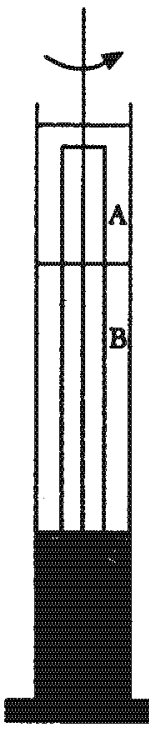

(c)

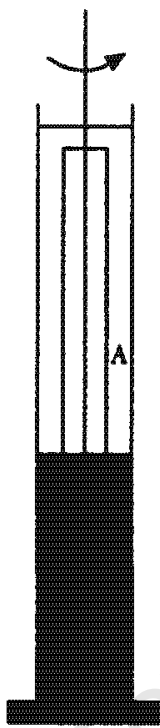

(d)

Figure 2.4: Chronological progress of a stirred batch test showing the different settling regimes in the column at the different stages of settlement.

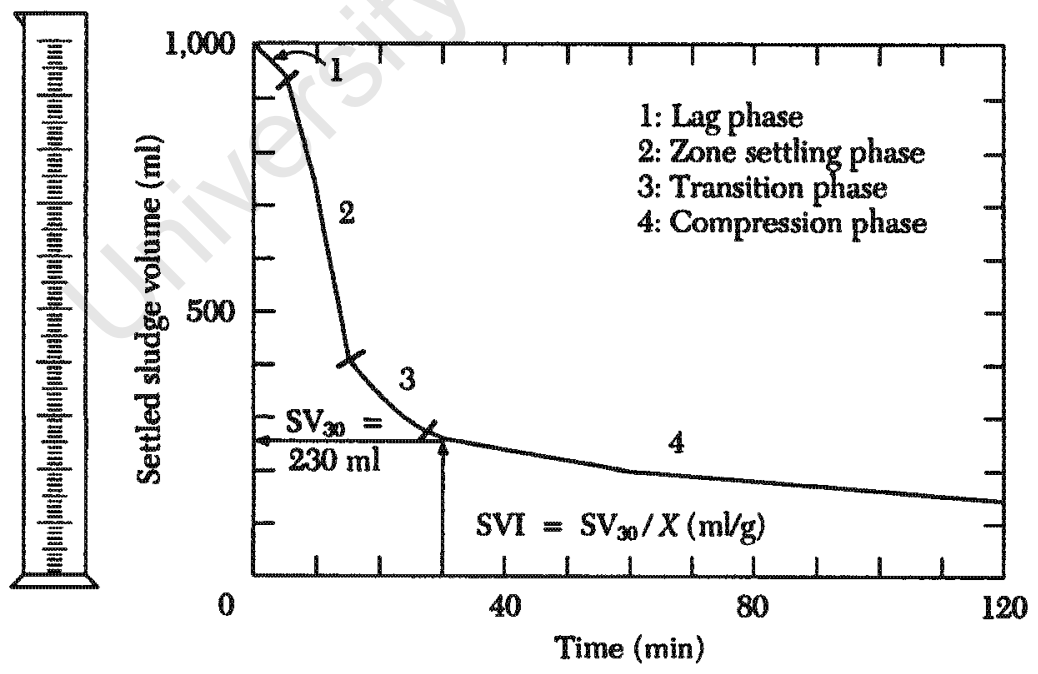

Figure 2.5: Plot of solid/liquid interphase depth against time in an unstirred measuring cylinder showing appearance of the different stages of settlement at the solid/liquid interface. 
respectively. While important for the ESS concentration, these two classes of settling are therefore largely ignored in the design of activated sludge SSTs.

The compression stage has not gained as much attention as the zone settling stage, although poor compression behaviour is often the reason for the failure of shallow SSTs with low underflow rates. The ATV (Abwassertechnische Vereinigung e.V., Germany 1991) includes this mode of failure in their design guidelines for SSTs (see Figure 2.4), while it is ignored in the flux-based procedures in the English-speaking countries. With the lack of knowledge and measurement methods for the compression behaviour of activated sludge, computational models cannot represent the sludge bed in an adequate way.

\subsubsection{Sludge volume indices}

The measurement of sludge volume indices is based on the volume approach and is not related to the solids settling velocity. The volume of sludge occupied after a fixed period of settlement, divided by the initial concentration, gives indirect but relevant information about the settleability of the activated sludge (Figure 2.5).

The sludge volume indices are given in $m \ell / \ell$ sludge volume per $g / \ell$ sludge concentration. Three main methods have been developed:

The Sludge Volume Index (SVI): The Sludge Volume Index is defined by

$$
S V I=\frac{S V_{30}}{X} \quad[m \ell / g]
$$

where $S V_{30}$ is the volume of sludge in a one-litre unstirred measuring cylinder after 30 minutes of settlement $[\mathrm{ml} / \ell]$, and $X$ is the sludge concentration [gMLSS/l] (Mohlman 1934).

The $S V I$ is the most commonly used test to obtain information about the settleability of activated sludge (Standard Methods 1985). Nevertheless, one of its crucial deficiencies is its dependence on the sludge concentration: above a certain concentration the $S V I$ increases with further increase in concentration, and thereafter decreases again.

The Diluted Sludge Volume Index (DSVI): Due to the deficiency of the SVI the Diluted Sludge Volume Index $(D S V I)$ has been developed. It is defined as the ratio of the volume of sludge in a $1-\ell$ unstirred measuring cylinder after $30 \mathrm{~min}$ 
settlement, to the sludge concentration, with the proviso that the settled volume after 30 minutes, possibly after dilution, is between $150 \mathrm{~m}$ and $250 \mathrm{~m} \ell$ (Ekama \& Marais 1984). The insensitivity of the DSVI to sludge concentration allows the comparison of sludge settleability in different activated sludge plants, and with it the integration of the DSVI into design procedures for SSTs such as those proposed by the ATV (1993) in Germany, Stofkoper \& Trentelman (1982) in Holland, and Koopman \& Cadee (1983) in the USA, who linked the DSVI to the flux theory predicted maximum solids loading rate on the settling tank.

The Stirred Specific Volume Index (SSVI): In order to reduce wall effects, shortcircuiting, and bridge formation effects in the settling column, and to create conditions more similar to those in the sludge blanket of SSTs, White (1975) proposed that the sludge be stirred during settling, and introduced the Stirred Specific Volume Index at $3.5 g M L S S / \ell\left(S S V I_{3.5}\right)$. This measure also reduces the effect of concentration. He found that for very poor settling sludge the SSVI is dependent on the initial sludge concentration, and suggested a standard concentration of $3.5 \mathrm{~g} / \ell$ for reporting $S S V I$ data. A number of tests have to be carried out over a range of sludge concentrations from 2 to $6 \mathrm{~g} / \ell$ in order to interpolate the value at $3.5 \mathrm{~g} / \ell$ from a $S S V I$ versus concentration graph; and a nearly linear relation between sludge concentration and settled sludge after $30 \mathrm{~min}$ in a stirred SVI cylinder occurs and justifies interpolation of the standard $S S V I_{3.5}$ at $3.5 \mathrm{~g} / \ell$

\subsubsection{Zone settling velocity}

The zone settling velocity can be determined experimentally in a batch cylinder with concentrations greater than $1 \mathrm{~g} / \ell$, as shown in Figure 2.5. This batch cylinder is usually $0.6 \mathrm{~m}$ in height and $0.1 \mathrm{~m}$ in diameter White (1976). Pitman (1984) used a larger column (1.5 $\mathrm{m}$ high and more than $0.2 \mathrm{~m}$ in diameter), and obtained similar results to White (1976) (Ekama \& Marais 1986). The subsidence velocity of the liquid/solid interface is a function of the concentration with which the column was filled. By means of several batch tests with different concentrations a settling function can be constructed that defines the relationship between the settling velocity and concentration. This relationship can be approximated by the exponential function (Vesilind 1968)

$$
V_{S}=V_{S o} \cdot e^{-n X}
$$


According to Parker, Kaufman \& Jenkins (1971) the particle size distribution of activated sludge is bimodal; that is, it comprises a group of small particles of particle size $0.5 \mu \mathrm{m}$ to $5 \mu \mathrm{m}$, and a group of large particles in the range 25 to $2000 \mu \mathrm{m}$. Relatively few particles are found in the range 5 to $25 \mu \mathrm{m}$. The large particles aid a high settling velocity and good thickening function, and the smaller particles are responsible for the effluent suspended solids (ESS) concentration. Therefore the more small particles that can be enmeshed and flocculated with the large particles and settled out, the lower the ESS concentration will be.

The exponential settling function (2.2) covers the concentration range in which hindered or zone settling takes place $(1<X<12$ to $15 \mathrm{gSS} / \mathrm{\ell})$, and is not valid for the dilute settling classes I and II, and the compaction class IV. While equation (2.2) provides an approximate approach to simulating compaction because the 'settling velocity' decreases with increasing concentration, (2.2) deviates completely for classes I and II settling, because the solids settling velocity continues to increase exponentially as the concentration decreases, and reaches a maximum at zero concentration. The lower concentrations comprise the smaller particles with slow settling velocity characterized by classes I and II settling.

Various approaches have been made in order to extend the settling function to lower concentrations. For example, Dupont \& Henze (1992) considered the sludge as a mixture of macroflocs with a settling function according to (2.2), together with microflocs that are responsible for the clouding of the effluent due to their low settling velocity. Takács et al. (1991) used an empirical double-exponential function

$$
V_{S}=V_{S o} \cdot e^{-n_{A}\left(X-f_{n o} X_{A T}\right)}-V_{S 0} \cdot e^{-n_{2}\left(X-f_{n o} X_{A T}\right)}
$$

to describe the settling velocity over the whole concentration range. Here $X_{A T}$ is the reactor suspended solids concentration, $f_{n s}$ is the non-settleable particle fraction, $n_{4}$ is an empirical coefficient for zone settling flocs, and $n_{2}$ an empirical coefficient for the dilute settling particles. The coefficient $n_{4}$, which is identical to $n$ in equation (2.2), is generally an order of magnitude larger than $n_{2}$, and the indices 2 and 4 refer to regions 2 and 4 in Figure 2.5, which illustrates generally the basis of Takacs' settling approach. The regions are defined as follows:

Region 1: Non-settleable particles in the concentration range 0 to $f_{n s} \cdot X_{A T}$.

Region 2: In this range the activated sludge is dilute and flocculent and the settleability improves with increasing concentration. The second term mainly 


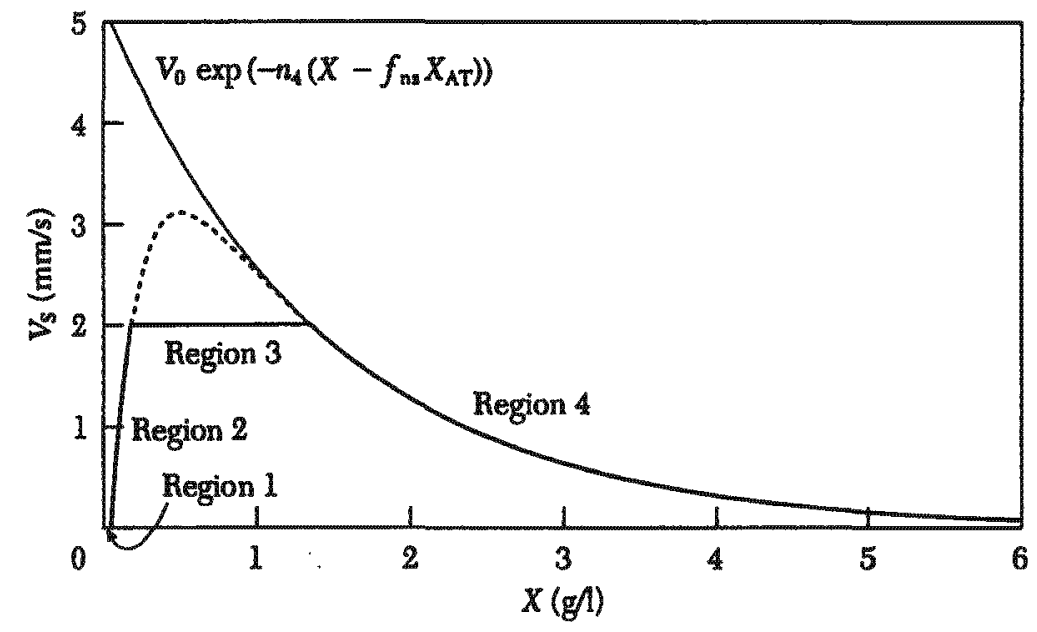

Figure 2.6: Settling function of Takács et al. (1991) incorporating the settleability of both dispersed and flocculated suspended solids.

describes this region.

Region 3: In this region it is assumed that the settling behaviour is nearly independent of the concentration.

Region 4: This region corresponds to the classical zone settling function (2.2) and is covered by the first term.

While equation (2.3) is a significant improvement over (2.2), the coefficient $n_{2}$ and the maximum settling velocity $V_{S 3}$ of Region 3 cannot be measured ( $f_{n s}$ possibly can be measured from the residual solids concentration in the supernatant after two to three hours settling). Here the classical classes I and II approaches as applied to water treatment sedimentation tanks are also not helpful. Therefore, while equation (2.3) extends the settling velocity versus concentration equation to low concentrations, it cannot be validated for the ESS that it is designed to predict. So by necessity, the $n_{2}, V_{S 3}$ and $f_{n s}$ values have been selected to match measured ESS concentrations on SST stress test trials. Clearly, methods to measure the dilute settling velocity behaviour need to be developed.

In order to avoid the time-consuming and tedious multiple batch zone settling tests to obtain the $V_{S o}$ and $n_{4}$ values, and yet still to be able to use the class III settling based on one-dimensional idealized flux theory for SSTs, several researchers developed empirical relationships linking the $V_{S}$ or the $V_{S o}$ and $n_{4}$ 


\begin{tabular}{|l|l|}
\hline $\begin{array}{l}\text { Hydraulic load features and ex- } \\
\text { ternal tank dimensions }\end{array}$ & Internal physical features \\
\hline \hline Wastewater flow & Presence of a flocculation zone \\
\hline Surface area and overflow rate & Sludge collection arrangement \\
\hline Depth and retention time & Inlet arrangement \\
\hline Underflow recycle ratio & Weir type, length and position \\
\hline & Tank configuration and baffling \\
\cline { 2 - 3 } & $\begin{array}{l}\text { Hydraulic flow patterns and turbu- } \\
\text { lence }\end{array}$ \\
\cline { 2 - 3 } & Density and convection currents \\
\hline \hline Sludge characteristics & Site conditions \\
\hline \hline Biological reactor concentration & Wind and wave action \\
\hline $\begin{array}{l}\text { Flocculation, settling and thickening } \\
\text { characteristics }\end{array}$ & Water and air temperature variation \\
\hline $\begin{array}{l}\text { Biological processes, e.g. denitrifica- } \\
\text { tion }\end{array}$ & \\
\hline
\end{tabular}

Table 2.2: Factors affecting solid/liquid separation in SSTs, adapted from Ekama et al. (1997).

values to simple sludge settleability parameters (for example, Ekama \& Marais (1986), Wahlberg \& Keinath (1988), Härtel \& Poppel (1992), and many others). A comprehensive list and a critical review of these relationships is given by Ekama et al. (1997). These researchers point out that great caution should be exercised in using any of the relationships, and that they should be restricted as far as possible to the type of activated sludge plants on which the data set was measured.

\subsection{Types of SSTs}

Clarification, thickening and storage functions of SSTs are mainly influenced by the wastewater flow and the sludge characteristics. Other factors affecting these aspects are physical features, such as inlet and sludge collection arrangements, as well as site conditions, such as wind and temperature. All these factors, which are important for efficient settling tank performance and low effluent suspended 
solids concentration (ESS), are listed in Table 2.2, adapted from Fiknna et al. (1997).

Generally, secondary settling tanks are classified according to the shape of the basirn, the main flow direction, and the scraper system. The basic configurations are rectangular and circular as well as vertical, and are described in the following sections.

\subsubsection{Rectangular secondary settling tanks}

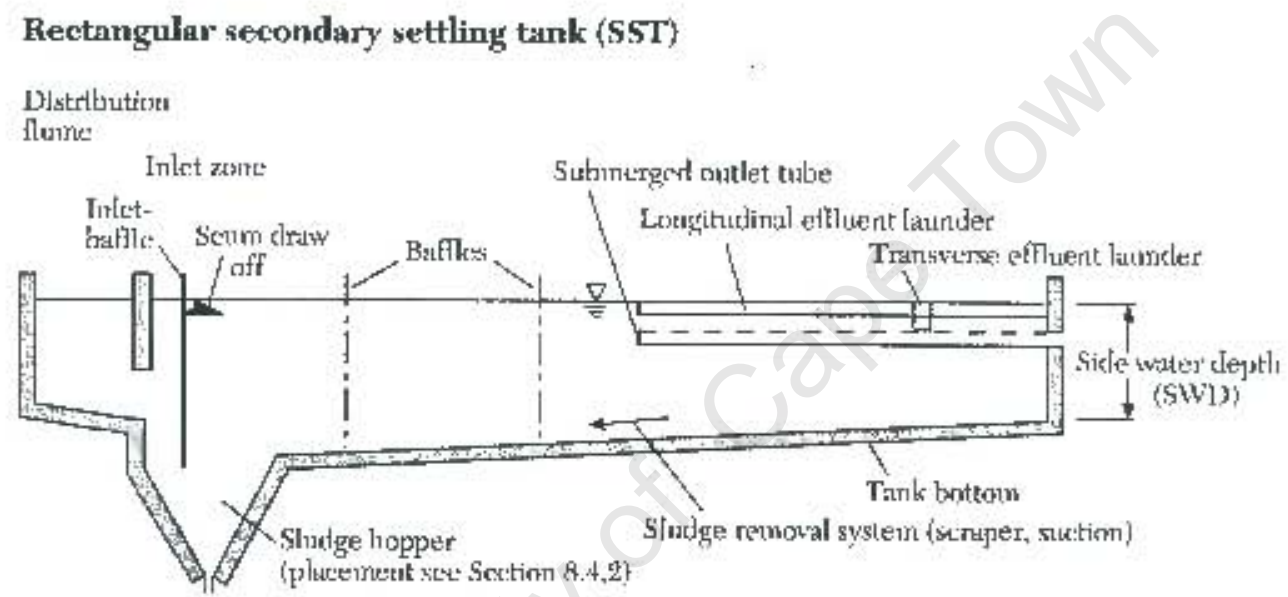

Figure: 2.7: Rectangular secondary settling tank.

Due to their apparent space-saving arrangements rectangular SSTs arc sometimes preferred when shortage of land area is an isst1e to be addressed. $\Lambda$ typical rectangular SST is shown in Figure 2.7. Depending on their main flow direction rectangular SSTs can be divided into longitudinal and transverse flow tanks. Longitudinal tanks have a relatively high ratio of loud to width of the tank. Guidelines such as those in the ATV (1991), suggest that the ratio of length to width should be at least 5 , with lengths of 1 sually 30 to $60 \mathrm{~m}$. The sludge removal systrems for this configuration type are generally blade or flight scraper systems, which transport the sludge to a hopper for further thickening or suction collectors equipped with a purnp. The removal takes place either with (concurrent) or against (countercurrent) the main flow. The ATV recommends that longitudina, SSTs should not exceed $60 \mathrm{~m}$ in length in order to avoid too low sludge remotal frequencies. 


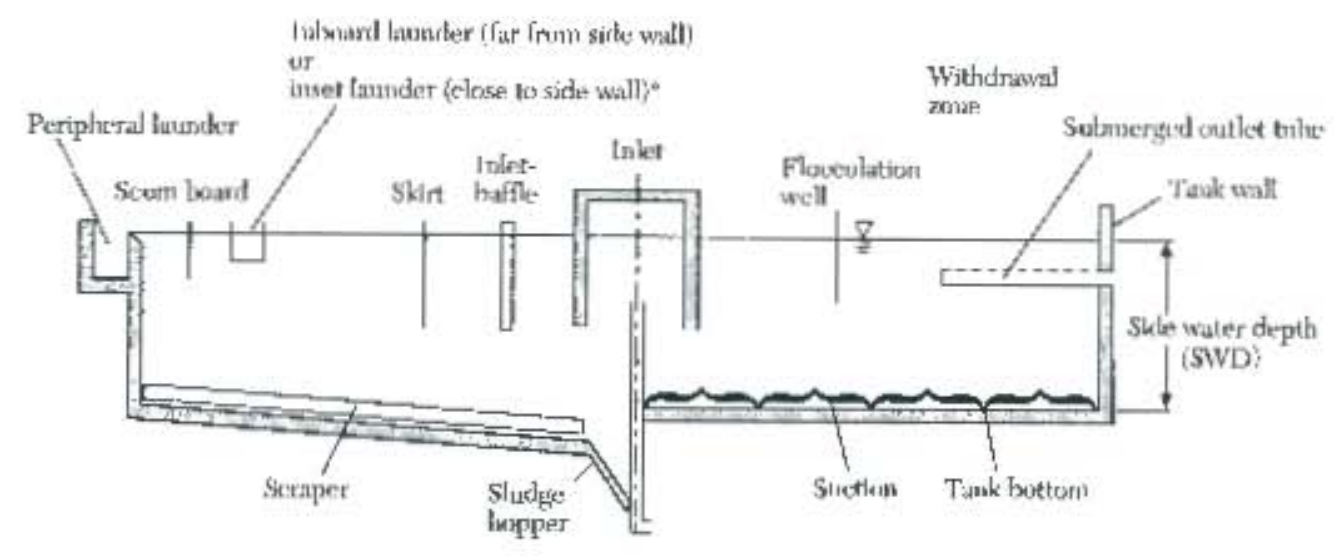

Figure 2.8: Centre fced, peripheral take-off, secondary settling tank.

In trunsverse flow tanks the inflow takes place over the longitudinal side of the tank. Lengths of up to $100 \mathrm{~m}$ make it difficult to achieve a uniform distribution over the whole side, which is significant and essential in the case of short horizontal flows: the typical width of such tanks is around $10 \mathrm{~m}$. Vertical flow tanks ure a special category, in which the ratio of width to water depth is of the order of two, and the inlet is placed at the bottom. Transverse tanks are combined with suction systems and, in contrast, to longitudinal tanks, the sludge removal is perpendicular to the muin flow.

\subsubsection{Circular secondary settling tanks}

The worldwide popularity of circular secondary settling tanks with centre feed (sce Figure 2.8) can be ascribed to the simple idea that the velocities continuously decelerate from the central inlet to the peripheral effluent outlet due to the opening flow profile, thereby improving the conditions for sedimentation. But, due to the fact that the specific loading per $m$ inlet is higher, circular and rectangular tanks are also affected by short circuit flow (Krels 1991b). Rcctangular and circular tanks have much in common, and the design procerlures and operation strategies are basically the same. The sludge removal is carried out by blade scraper systems on a sloped bottom and a central hopper, or with suction collectors. 
The dovelopment of peripheral feed SS'Ts was motivated by the fact that converging flow is more stable than the diverging flow of centre feed circular clarifiers. In addition, the inlet area is much larger, which reduces influences on the flow from density currents. Although the hydraulic efficiency may be better, the peripheral SST has not gained much attention in current practice. Reasons include the difficulty to ensure equal flow distribution with varying flows, and the problems with foam that are experienced particularly in peripheral feed tanks. The author is aware of two wastewater treatment plants that have changed their peripheral-fecd SST's to centre-feed SST's to improve their capacity.

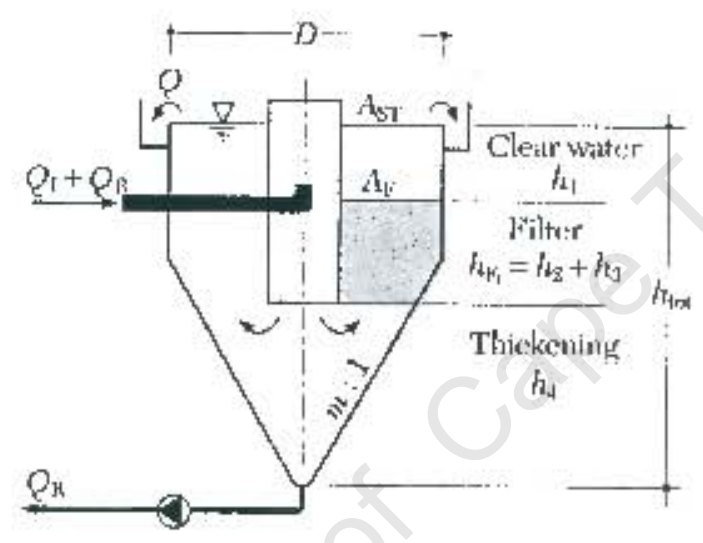

Figure 2.9: Dortmund tank.

\subsubsection{Vertical secondary settling tanks}

This specially designed tank has boen constructed mainly in Germany. It is of large water depth aud relatively small surface area, and the inflow takes place within the sludge blanket in order to filter fine particles from the upflow, as iflustrated in Figure 2.9. The foc filter dewelops at the lovel where the settling velocity of the activated sludge and velocity of the upfow are in balance. Consequently, the floc filter reacts very sensitively to variable loading conditions. In order to overcome this difficulty these tanks are often designed as hoppers. Duriug higher inflows the upward velocity decreases towards the top with the increasing surface, and a new equilibrium situation can develop. The first vertical tank, called the Dortmund tank, was already built at the end of 1900 (Dunbar 1954). In general this type of tank is attractive when the loading couditions are 


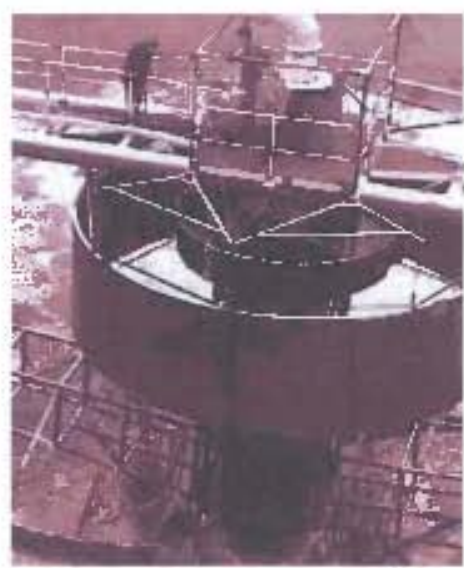

Enlet chamber

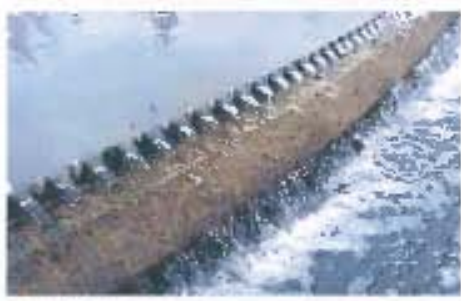

Effluts! wcir

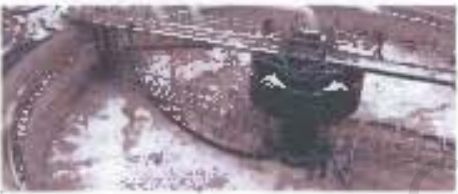

Scrapel' tor the recycled sludge

Figure 2.10: Typical features of a centre-feed circular SST.

expocted to be very stable.

\subsection{SST configurations}

The most important SST features are the inlet, the effluent outlel., and the sludge removal mechanism. A series of photographs in Figure 2.10 show these in a real circular SST. This section will bricfly discuss the different SST contigurations, and will give examples used in practice. A comprehensive list and it critical review is given in Ekama et. al. (1997).

\subsubsection{Inlet}

In general the inlet structure should support the separation of aclivated sludge: in the sedimertation region. This can bo achieved by considering three main 
criteria (Krebs 1991a):

(i) Kinetic energy and pressure of the inflow should be minimized and be well distributed over the whole width of the tank. Inlet structures such as lamellas, perforated walls, diffusers and stem inlets with baffles were traditionally used to dissipate inlet energy as well as to induce head loss and distribution effects. The reduction of the maximal inlet velocity of around $1 \mathrm{~m} / \mathrm{s}$ to a few $\mathrm{cm} / \mathrm{s}$ causes a huge energy loss (WPCF 1985), which can result in floc break-up. In order to avoid this effect lamellas and perforated walls, for example, should be positioned sequentially, and the width of the openings should not be too small.

(ii) The potential energy of the inflow should be as small as possible. Classical inlet structures ignored the density effect and the inherent high potential energy. High vertical or mid-depth apertures cause a waterfall effect in the inlet region due to the density influence. The potential energy is reduced by installing the inlet section at a low position. Suggestions have been made to position the inlet over the thickening zone in order to avoid turbulence. In-situ measurements of Deininger (1997) and numerical simulations of Lakehal, Krebs, Krijgsman \& Rodi (1999) have shown that the main flow current always became established at the upper boundary of the sludge blanket as long as the flow field was developed and steady state. On the other hand, the sludge blanket can operate as a floc filter when introducing the inflow in areas of high concentrations. Otterpohl \& Freund (1992) observed that the concentration of non-settleable particles decreased with increasing sludge concentration in the aeration tank. Nevertheless, the effect and consequence that will dominate is still unknown.

iii) The potential of the activated sludge to flocculate should be used and maximized by designing the inlet as a flocculation chamber. The required residence time for optimal flocculation determines the volume needed. On the basis of laboratory and in-situ experiments at average dry weather flows an average residence time of $20 \mathrm{~min}$ has been suggested (Wahlberg et al. 1994).

Another important function of such flocculator wells or skirt baffles was found by Zhou \& McCorquodale (1992a,b). In their model simulations they showed that most of the entrainment of flow between the influent and the ambient fluid in the tank occurred in the density waterfall, and that with the aid of skirt baffles this entrainment compensating flow could be reduced. 


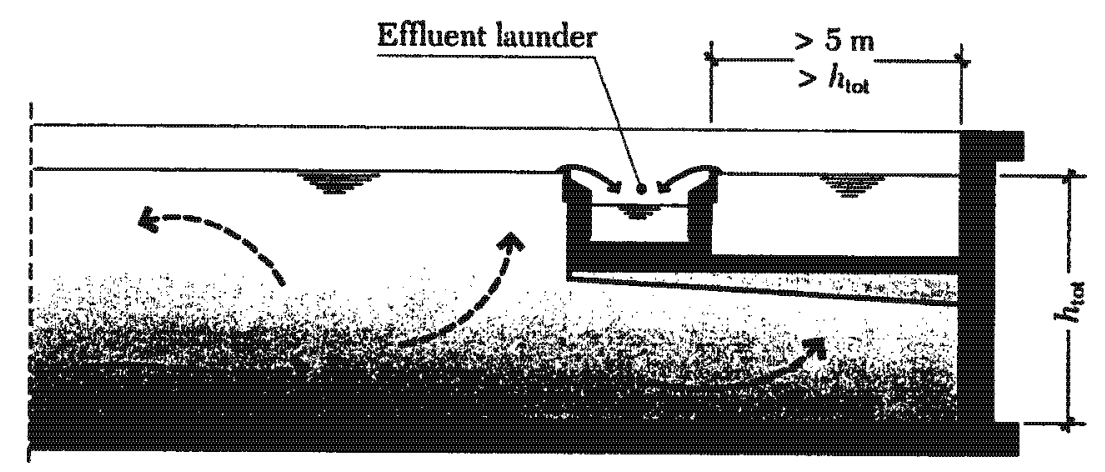

Figure 2.11: Arrangement of an inboard launder.

\subsubsection{Outlet}

Two different outlet structures have been established for both circular and rectangular tanks:

Effluent launders: Most effluent launders are positioned at the periphery of the tank, simply because they have the largest distance to the inlet. But the deflection of the density current at the end of the tank causes suspension clouds to rise, and for this reason the weir or effluent launder should not be placed at the periphery of these tanks. It has to be emphasized that it is the excess energy from the density current at the bottom, and not the suction effect of the launder, which actually is only predominant in the near-field, that causes the concentrated upflow at the outside wall. The ideal situation is a homogeneous withdrawal, which can be obtained by using several launders between the extended inlet region and the region close to the end wall (Figure 2.11).

Submerged outlet tubes: With the installation of longitudinal or radially orientated submerged outlet tubes, or several transverse or concentric tubes, a more uniform withdrawal from the effluent surface area is achieved. Gunthert \& Deininger (1995) investigated the advantages of submerged tubes and reported a more stable behaviour to variations in water depth compared with surface effluent weir launders. This can be ascribed to the fact that the flows through orifices under pressure result in a more uniform and stable effluent flow distribution. 
(a)

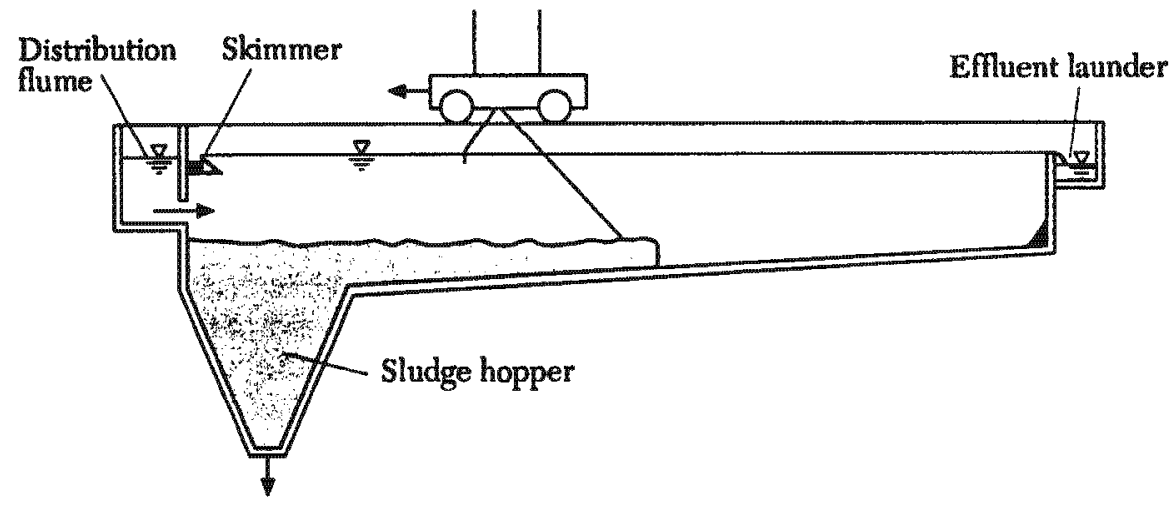

(b)

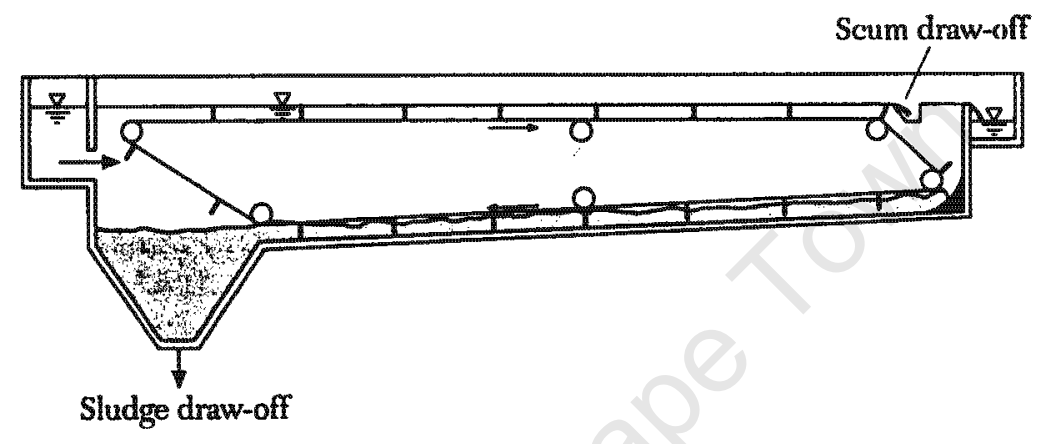

Figure 2.12: Sludge removal systems for rectangular SSTs: (a) blade scraper system, (b) flight scraper system.

\subsubsection{Sludge removal}

There are two categories of sludge removal systems: scraper systems, like bladeor flight scraper systems (Figure 2.12), and suction collectors. It is still unknown which sludge removal system provides the better solution. Wahlberg et al. (1993), for example, found that the use of suction collectors led to a deeper sludge blanket and a lower ESS concentration. In SSTs with scraper systems the thickened sludge has to be transported along the bottom to the sludge hopper, whereas in SSTs with suctions lifts the sludge is removed where it settles, and resuspension is far less likely. On the other hand suction collectors may be less efficient due to passible withdrawal of the surrounding dilute fluid. In any event, both sludge removal systems loosen up the compacted sludge bed, which facilitates sludge removal. 


\subsection{Closure}

The design of SSTs focuses on the zone settling behaviour of the activated sludge, from which the required surface area is obtained. Tank depth is designed empirically from sludge storage and compaction requirements, and SSTs are usually between 3 and $5 \mathrm{~m}$ side water depth. The sludge settling velocity versus concentration behaviour, which is required in the flux theory model for SSTs, can be measured only in the zone settling region. Whether the well-established zone settling velocity versus concentration equation applies to compaction at the bottom of the SST is uncertain, but at least the settling velocity decreases as concentration increases, as expected of the compaction stage. However, the equation is a poor model for settling behaviour at dilute concentration. The modification proposed to extend the solids settling velocity versus concentration equation to dilute concentration, while performing as intuitively expected in this region, lacks an experimental basis, and therefore cannot predict the effluent SS concentration (ESS) for which it was developed. The situation has developed that the SST external shell is designed by means of the flux theory and the associated zone settling velocity-concentration behaviour of the sludge, which is well founded on experimental measurement. The external shell so specified is then equipped with all kinds of internal features, which under daily varying hydraulic and solids loading rates (1) maintain a low ESS with baffes, launder position and size, flocculation chambers/wells, (2) keep the sludge blanket low with adequate sludge transport and withdrawal, and (3) maintain high underflow concentration by minimizing short circuiting and sludge blanket disruption by hydraulic disturbances.

Internal features aimed at achieving these have been briefly reviewed above. It would seem that while some of these features can be incorporated in hydrodynamic models, the greatest shortcoming in hydrodynamic modelling is the relationship linking the sludge settling velocity to concentration across the full spectrum from dilute discrete settling behaviour to concentrated compaction behaviour.

The modelling of hydraulic flows in SSTs is reviewed in the next chapter. 


\section{Chapter 3}

\section{Experiments and numerical modelling related to flows of SSTs}

Two approaches have been used to achieve improvements in the design of settling tanks: (i) experimentation, and (ii) computational fluid dynamics (CFD). Relevant studies in these two areas are described below.

\subsection{Experiments}

Experiments can be performed in three different ways: (i) prototype experiments under real conditions; (ii) pilot scale tests with a model tank being fed by activated sludge; or (iii) laboratory-scale experiments using an artificial test suspension.

Laboratory-scale experiments are used mainly to validate computational flow simulations and to investigate the influence of different inlet and outlet configurations. Pilot-scale experiments are necessary to study the flow behaviour in the settling region, which is defined by the characteristics of activated sludge. Prototype experiments are very difficult to carry out due to varying boundary conditions with time, such as for variations of the inflow and continuously changing conditions due to the scraper system in the tank. Additionally, the presence of small velocities and the wide range of sludge concentrations in connection with a 
long measurement time make the experiments difficult to control. Nevertheless, due to the fact that the behaviour of SSTs can only fully be understood with the interaction of the biological reactor and, unlike all other parameters and dimensions, activated sludge cannot properly be scaled down; prototype experiments are therefore the only realistic option. We discuss some of these in the following section.

\subsubsection{Prototype experiments}

Pioneering work on circular secondary settling tanks was carried out by Anderson (1945). With the use of a drifting body Anderson measured the velocities and suspended solids concentrations in a centre-fed circular secondary settling tank, and showed at first that the flow pattern was different from that in other settling tanks: in the primary settling tank (PST) a current along the surface from inlet to outlet was observed, while a strong density current at the bottom and a compensating reverse current in the upper zone of the settling zone developed in the secondary settling tank (SST) (Figure 3.1). The significant difference could be ascribed to the fact that the particle concentration in a primary clarifier is small compared to that in a SST, and therefore the solids have little influence on the flow pattern in the tank.

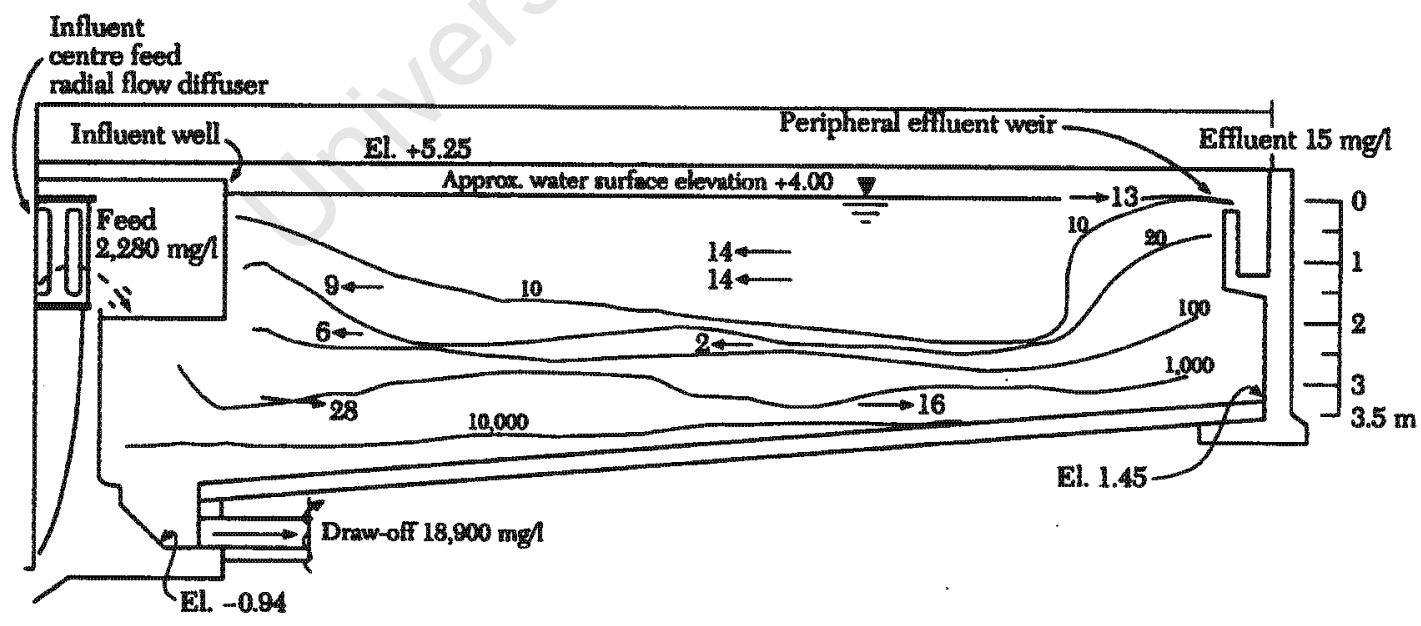

Figure 3.1: Flow pattern and solids distribution in a circular secondary settling tank (Anderson 1945). The tail of each arrow indicates the start of a velocity reading. Velocities are shown in $\mathrm{mm} / \mathrm{s}$. 
Due to the lack of appropriate measuring devices in the decades that followed, hydraulic efficiency was studied with the use of tracers such as dye, salt, or radioactive tracers. The measured flow-through could give information about the influence of geometry and inlet arrangement, as well on whether short-circuiting was taking place.

In short-circuiting studies a tracer is injected into the influent, and the concentration of the added substance in the effuent is observed at the end of various time intervals until substantially all the suspended solids have passed from the tank. From the data obtained, a flow-through curve (FTC) can be constructed in which the concentration of the tracer against the time is plotted (Figure 3.2). These curves in dimensionless terms allow comparisons between the hydraulic characteristics of different shapes of tanks, or of the same tank at different rates of discharge.

The tanks found in practice will be intermediate to the two extreme types of tanks (see also Figure 3.3): the ideal mixed reactor, in which the influent is dispersed instantaneously and uniformly throughout the tank volume (curve A in Figure 3.2) and the ideal plug flow reactor in which the velocity is constant throughout the tank (vertical line at $t / T$ equal to unity $(F)$ in Figure 3.3). The ideal plug flow is the most efficient for sedimentation, while the ideal mixed reactor is the least efficient.

Camp (1946) carried out tracer tests on different tanks, and the flow-through curves are shown in Figure 3.2. The figure shows the measured and normalized tracer concentration $c / c_{o}$ at the outlet against the measured time $t / T$ with $T$ as the theoretical retention time. Curve $B$ represents the flow pattern of a circular primary tank with the conventional type of inlet, curves $\mathrm{C}$ and $\mathrm{D}$ are the result of a wide and narrow rectangular tank, respectively, and curve $\mathrm{E}$ is the flowthrough curve of a round-the-end-type baffled mixing chamber, which indicates what might be expected of well-designed tray clarifiers. The closer the measured flow-through curve is to that of an ideal plug flow $(F)$ the better the utilization and flow-through of the tank are. A flow-through curve close to that of an ideal mixed reactor is an indication for the existence of short-circuiting flow and of dead zones in the tank.

Larsen (1977) carried out extensive measurement tests on the velocities in rectangular tanks with the use of an ultrasonic probe (Figure 3.4). In addition, 


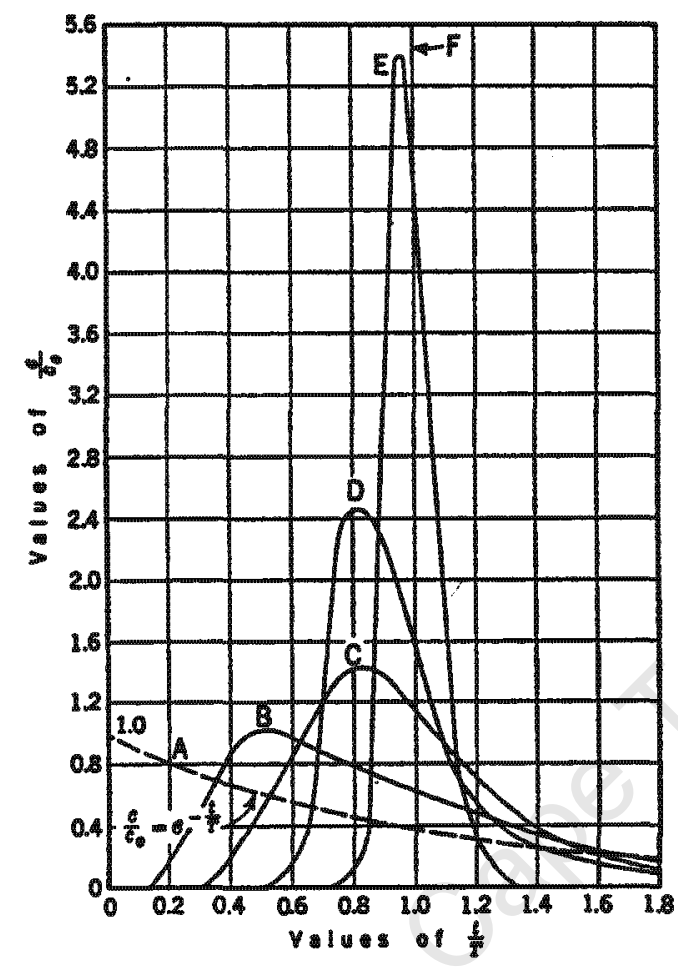

Figure 3.2: Typical flow-through curves for tanks (Camp 1946).

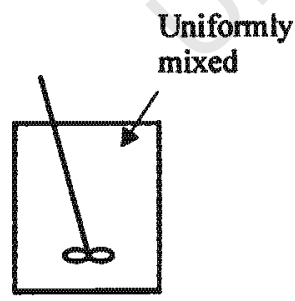

Batch reactor

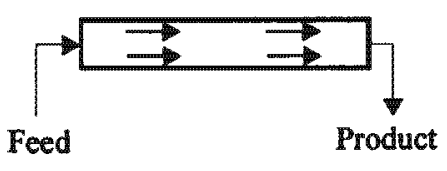

Plug flow reactor

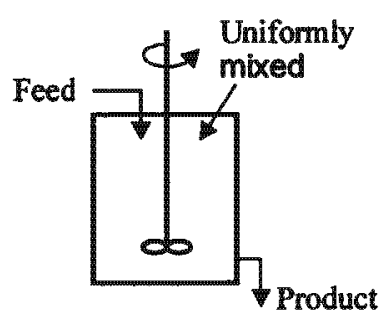

Mixed flow reactor

Figure 3.3: The three types of ideal reactors: batch reactor, plug flow reactor and mixed flow reactor. 


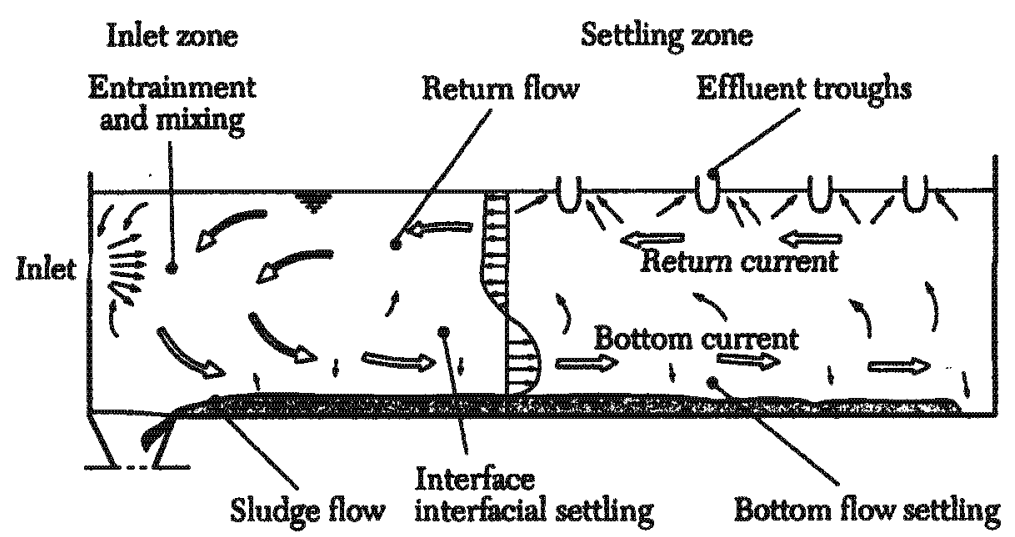

Figure 3.4: Typical secondary settling tank flow patterns found by Larsen (1997).

he presented measurements of velocity fluctuations and made energy balances to identify which inputs are decisive regarding the flow pattern. The ultrasonic probe, however, could not give any data in the high concentration range of the sludge blanket.

Bretscher et al. $(1984,1992)$ undertook measurements with drifting body techniques. They demonstrated experimentally the correlation between the distributions of velocity and solid matter. The results gave an impression of how strongly the flow could be influenced by inlet arrangements and baffles.

An ultrasonic probe was used by Gunthert \& Deininger (1995) to perform velocity measurements in a circular tank under conditions of dry-and wet-weather loading. They demonstrated that even under wet-weather loading conditions, the forward current still flowed over the sludge blanket, although the sludge blanket height had grown considerably.

\subsubsection{Pilot scale tests}

In pilot scale tests the advantages of prototype and laboratory scale experiments are combined. The relatively large tanks allow the use of activated sludge as test suspension, as well as the free choice of different boundary conditions, such as different loading conditions. This makes the measurements more controllable. Nevertheless, due to the high expenditure associated with pilot scale equipment, only a few such experiments have been carried out. 
Dahl et al. (1994) performed experiments on a model tank $10 \mathrm{~m}$ in length and $1 m$ in height, with the sludge taken from the wastewater treatment plant. An optical measuring instrument was used to measure the suspended sludge concentration, and an electromagnetic induction sensor was employed to measure the velocity components. Problems arose with regard to scaling down the thickening of activated sludge; that is, the ratio of sludge blanket height to water depth became too large in comparison with that in a realistic system.

Baumer, Volkert \& Krebs (1996) operated a bigger tank of length $15 \mathrm{~m}$ and water depth $3 \mathrm{~m}$, which neglected scaling effects. They investigated the influence of internal features in the settling zone.

\subsubsection{Laboratory scale experiments}

Laboratory scale experiments provide a mechanism for understanding the local influence of internal features of SSTs, and for verifying the results of numerical models. These model experiments must obey the laws of similitude in order for results to be applicable in real systems; that is, the model and the prototype must have geometric, kinematic and dynamic similarity. The latter requires also the scaling of the settling behaviour, which makes for the greatest difficulties.

With regard to the similitude, the laws of similarity apply:

1. the flow must be turbulent ${ }^{1}$. According to Simpson (1987) density current can be regarded as turbulent when $R e>1,000$. Here Re is the Reynolds number, defined by:

$$
R e=\frac{U \cdot H}{\nu},
$$

where $U$ is a characteristic velocity, which for SSTs is the inflow velocity, $H$ is a characteristic water depth, and $\nu$ is the kinematic viscosity;

2. the ratio of buoyancy and momentum should be equal in the model and in the prototype. In flows with density currents induced by different temperature or concentration distributions, the densimetric Froude number $F_{D}$ relates momentum to buoyancy or kinetic energy to potential energy per

\footnotetext{
${ }^{2}$ Although the velocities in the main section of settling tanks are very small, the large scale of these tanks together with the small kinematic viscosity of water render the flow turbulent.
} 
unit time, according to

$$
F_{D}=\frac{U_{I n}^{2}}{g \cdot\left(\Delta \rho_{I n} / \rho_{r}\right) \cdot H_{I n}} .
$$

Here $U_{I n}$ is the horizontal inlet velocity, $H_{I n}$ the inlet depth and $\Delta \rho_{I n}=$ $\left(\rho_{I n}-\rho_{r}\right)$ the difference between the inlet density $\rho_{I n}$ and the reference density $\rho_{r}$ of clear water;

3. the ratio of settling velocity to flow velocity should be equal in the model and in the prototype. Due to the fact that the activated sludge settles relative to the water flow, the similarity of the settling velocities in experiment and prototype must be fulfilled, so that

$$
\left(\frac{V_{S}}{U}\right)_{\text {model }}=\left(\frac{V_{S}}{U}\right)_{\text {prototype }} .
$$

With a slightly distorted ratio of the length $L$ to water depth $H$ the Hazen numbers $H a$ of the model and prototype should be equal, where

$$
H a=\frac{V_{S} L}{U H} .
$$

However, it is not possible strictly to fulfil the scaling law for the settling velocity $V_{S}$ over the whole concentration range, which includes flocculation, zone settling, transition and compression. The use of activated sludge is the only way to comply with the similarity rules. On the other hand, in order to fulfil the geometry similarity by scaling down the tank size, the sizes of the flocs have to be decreased as well.

On account of this difficulty, laboratory experiments have been carried out mainly on clear water models to evaluate construction details, or to verify numerical flow simulations.

Lyn (1992) and McCorquodale \& Zhou (1993) used inflowing salt water, and cold or warm water to take into account density effects. With these transient experiments the passing of the bottom current front and the reflected reverse flow front could be analysed.

With the use of clay powder Krebs (1991a,b) and Van Marle \& Kranenburg (1994) performed their experiments under steady state conditions. The laboratory tank of Van Marle et al. was a $60^{\circ}$ sector model of a circular centre-feed secondary clarifier with a geometric scale of about 1:8. The experiments showed 
that as soon as the influent became more concentrated, gravity changed the flow pattern completely. A three-layer structure developed in the basin, as illustrated in Figure 3.5a. The experiment with a much lower influent concentration showed an earlier rise in concentration, and a significantly higher final effluent concentration. The conclusion was drawn that a layered flow pattern as a result of gravity currents seemed to promote settling rather than hinder it, as had been generally assumed. A typical short-circuiting flow is illustrated in Figure 3.5b.
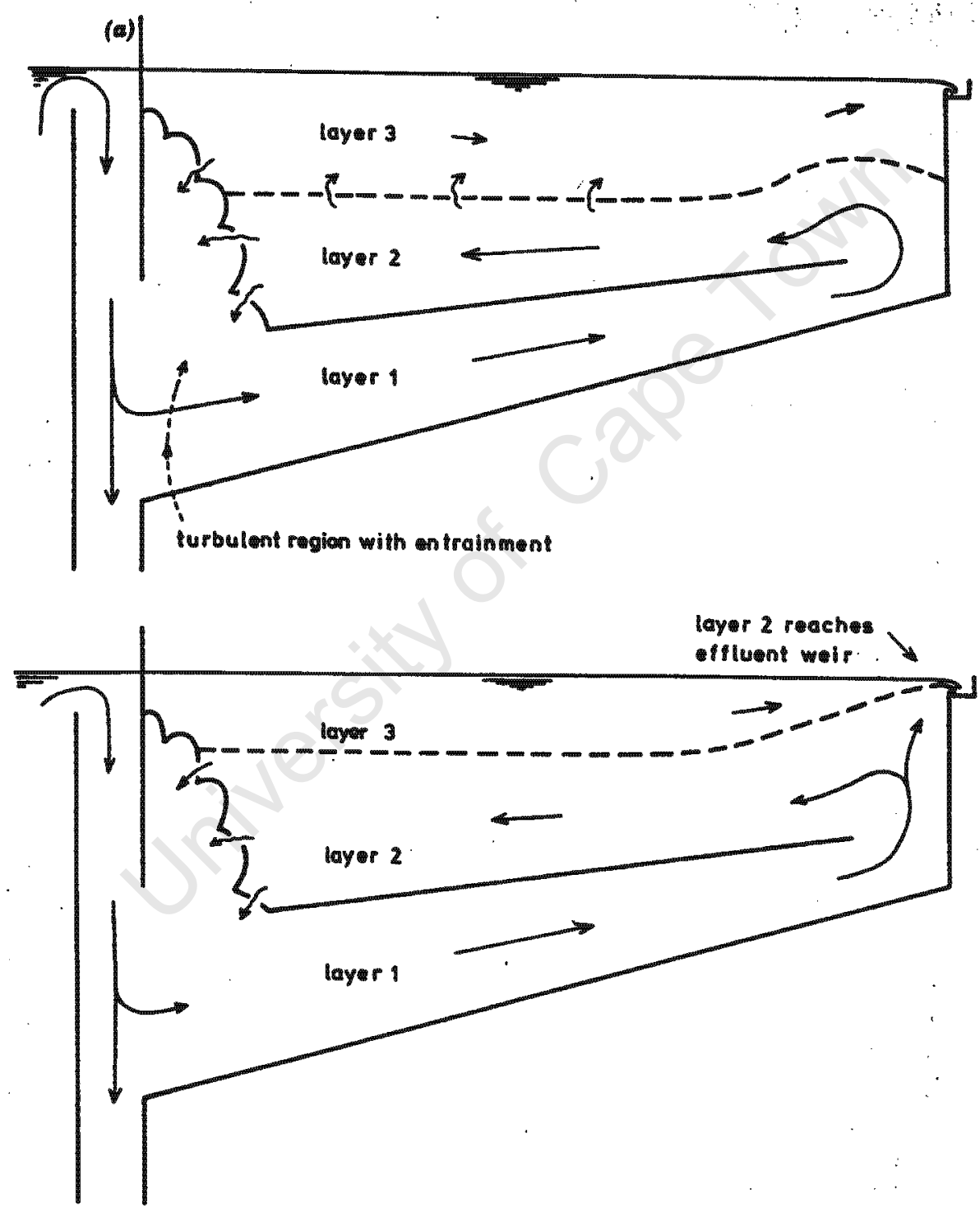

Figure 3.5: (a) Three layer-structure and (b) three layer-structure with shortcircuiting (Van Marle \& Kranenburg 1994). 
With the use of an ultrasonic probe Krebs (1991a,b) performed systematic experiments in a rectangular tank in order to determine the velocity and concentration fields and to investigate the density current and energy dissipation, and their dependence on different inlet structures. The model tank had a length of $5 \mathrm{~m}$, a width of $1 \mathrm{~m}$, and a water depth of approximately $0.5 \mathrm{~m}$. The results showed that the total energy input could be reduced by an inflow which was situated relatively low in the tank.

\subsection{Hydrodynamic modelling}

Various mathematical models for flows in SSTs of varying complexity and area of application have been developed over the last century. These include one-, two- and three-dimensional models.

Already in 1904 Hazen introduced an idealized assumption to describe the flow and sedimentation processes in rectangular settling tanks. Based on Hazen's approach the overflow rate concept was developed, according to which all particles with a settling velocity larger than the overflow rate can be settled to the bottom before reaching the end wall. This approach can describe approximately processes in large grit chambers and primary settling tanks, but is not applicable to secondary settling tanks due to the strong interaction of flow and sedimentation in these tanks.

In 1916 Coe \& Clevender conceived the flux theory, which is the mathematical basis for dynamic $1 \mathrm{D}$ modelling. With the appearance of the Activated Sludge Model ${ }^{2}$, extensive attention was paid to the dynamic 1D modelling of SSTs (Ekama et al. 1997). With the 1D activated sludge reactor-SST model the basic design and sizing of the various tanks can be achieved. The model addresses questions such as the return sludge concentration, the sludge blanket height, and solids inventory management.

Final design and optimization of tank and interior facilities require two- and three-dimensional models. Two-dimensional models seem to give reasonable results for circular tanks. However, if inlet and outlet structures are to be optimized

\footnotetext{
${ }^{2}$ The model gives a reliable description of the nitrification and denititrification system rosponse over wide ranges of system configuration of WWTPs, wastewater characteristics and operational parameters (Dold et al. 1991).
} 
in proper detail, three-dimensional models have to be considered.

\subsubsection{One-dimensional (1D) models}

A 1D model that is extensively used in English-speaking countries is that based on the flux theory of Coe \& Clevender (1916) and Kynch (1952). The flux theory specifies idealized concentration profiles in the settling tank at steady state. The settling tank is idealized as a continuous flow reactor, in which horizontal flow processes are neglected. The hydraulic detention time and the surface loading rate are taken from the prototype.

Starting at the inlet cross section, at which water and suspended solids enter the reactor, the flow is divided into a downward flow towards the underflow exit at the bottom and an upward flow towards the effluent exit at the top. Due to the settling motion of the suspended solids a sedimentation flux is added to the bulk flux. For the calculation the 1D tank is divided into horizontal layers at which the mass fluxes are formulated as mass balances.

The models developed, for example in Anderson (1981), represent progressive refinements in the development of a 1D flux-based dynamic model for secondary settling tanks. In the early 1990 s several models were presented, such as those in Dupont \& Henze (1992) and Ozinsky, Ekama \& Reddy (1994). With the appearance of the activated sludge simulation models, such as those in Henze, Grady, Guyer, Marais \& Matsuo (1991), the kinetics and modelling of the activated sludge biological reactor were very well advanced, and it became obvious that the most serious deficiency in modelling the activated sludge system concerned the modelling of the secondary settling tank.

In order to obtain reasonable results the correct modelling of the settling behaviour is a crucial point. Various attempts have been made to determine the settling velocity as a function of the sludge concentration and the sludge volume index (SVI) (see Section 2.4). Such settling approaches can be found for example in Wahlberg \& Keinath (1988). The required parameters of these settling functions are calibrated in sedimentation experiments under different activated sludge concentrations.

One-dimensional models give a reasonable approximation of the sludge balance (Ozinsky, Ekama \& Reddy 1994) and they require minimal computational ca- 
pacity. When simulating the sludge shift from the aeration tank to the SST in order to mimic sludge storage during wet-weather loadings, dynamic 1D models are suitable for coupling with activated sludge models (Diehl \& Jeppsson 1996). These 1D models are of second order and they contain a turbulent diffusivity term, which varies with SST depth and hydraulic loading, and which effectively reduces the actual SST hydraulic and solid loading rate capacity to less (70\%$85 \%$ ) than that given by the idealized 1D flux theory limit (Ozinsky, Ekama \& Reddy 1994). They are superior over the widely used 1D models of first order but rarely applied The incorporated diffusivity term needs to be calibrated for each SST, and therefore a lumped parameter, which seeks to take account of all the non-idealities, such as short-circuiting, density currents and the effects of the internal features in the actual SST, is included.

However, even when calibrated, 1D models cannot capture adequately the flows in a real SST, in which uneven velocity profiles, turbulence effects, short circuits, and the sludge removal result in fully three-dimensional behaviour.

\subsubsection{Two-dimensional (2D) models: theoretical and com- putational research}

Two-dimensional models of flows in SSTs are able to capture more accurately internal processes, and their influence on the functions of the secondary settling tank. The flow and sedimentation processes are generally simulated in a longitudinal section of a rectangular tank or a radial section of a circular tank, with the assumption that effects in the third dimension can be neglected.

The first numerical two-dimensional simulations of settling tanks were carried out on clear water conditions or with such low solid concentrations that the influence of the solids on the flow field could be neglected. Pioneering work was done by Larsen (1977) who studied the theory of settling tanks and described a mathematical model.

Schamber and Larock (1981) simulated settling tank flows using the finite element method applied to the coupled governing equations for fluid flow and concentration. The model included consideration of turbulence, which was accounted for by using the $k-\varepsilon$ model. Density and settling velocity effects were not taken into account. The planar model had a sloped bottom, one inlet, and 
one outlet, both at the water surface. The results of the computation showed the expected flow pattern of rectangular tanks without density effects. Schamber \& Larock used the finite element method to seek solutions in geometrically complex nonpolygonal domains and to avoid difficulties in applying boundary conditions on such domain boundaries. The stable quadratic velocity and linear pressure element was used. For more stable convergence the equations were partitioned into two sets to solve the mean flow equations and the turbulence equations alternately. A modified approach of Newton's method was applied to each set in turn. However, the sensitivity of the Newton-based solution algorithm to initial values was found to be excessive.

DeVantier \& Larock $(1986,1987)$ were the first to consider density effects. They carried out a finite element analysis of a 2D model with the dominant features of density-driven turbulent flow incorporated. They made use of settling velocity as a function of concentration; this relationship was obtained from laboratory column tests. The model was applied to a real circular secondary settling tank with a reaction baffle at the inlet. The inlet concentration had to be limited to maximum of $1400 \mathrm{mg} / \ell$ due to numerical instabilities caused by steep concentration gradients. Additionally, the solution of the flow in the inlet region itself caused strong numerical instabilities along the baffle, and as a result the inlet was modelled by a uniform horizontal velocity field below the baffle. In their finite element code DeVantier \& Larock used the same mixed weighting of the residuals as in (Schamber \& Larock 1981). In order to account for the strong dependence of pressure and concentration the partitioning of the variables into the two subsets of velocity components, pressure and concentration and of turbulent kinematic energy and its dissipation, was made. The Newton-Raphson method was used to solve the set of nonlinear equations. However, the strong non-linearity of the turbulence variables caused divergent results and modified forms of the Newton coefficient matrix were required.

Stamou, Adams \& Rodi (1989) considered several particle classes, with corresponding settling velocities, but did not incorporate density effects. They modelled a shallow rectangular primary settling tank. The inflow was uniform and positioned at the bottom, with the outlet at the water surface. Although concentration profiles and the rate of sludge removal were in good agreement with measurements, the flow-through curves of the model showed significant differences to those obtained experimentally. The code of Stamou et al. (1989) was based 
on the finite-volume procedure TEACH ${ }^{3}$ (Gosman \& Pun 1974). The pressurevelocity coupling was done using the SIMPLEC ${ }^{4}$ algorithm in VanDoormal \& Raithby (1984). The hybrid scheme ${ }^{5}$ was applied to the convective terms in the momentum equations and the QUICK ${ }^{6}$ scheme to those in the concentration equation, in order to reduce numerical diffusion. For predicting the flow-throughcurve a fully implicit first order-accurate temporal discretization was employed.

Adams \& Rodi (1990) investigated the influence of different approximations of the convective terms in the concentration equation either by using the hybrid central/upwind scheme or the more accurate third-order QUICK scheme. The results with the QUICK scheme were significantly better compared to those with the hybrid scheme, which were traced back to the diffusive side-effect from truncation errors, so-called numerical or false diffusion. However, the peak concentrations of the flow-through-curves were overpredicted with the QUICK scheme and underpredicted with the hybrid scheme.

Lyn et al. (1992) implemented the gravity-density term in the vertical momentum equation in order to describe the sediment-induced density currents. The qualitative agreement between predictions and measurements were markedly improved. In Figure 3.6 the effect of density differences is clearly seen. The numerical model was applied to a real tertiary, rectangular settling tank. The inlet was approximated as a slot jet inlet, and the sludge removal was carried out by a hopper modelled through the use of a nonorthogonal grid (Figure 3.6). The code in Lyn et al. (1992) was an adaptation of a code developed by Majumdar (1986), which resembled in many respects the code used by Stamou \& Adams, but permitted the use of non-orthogonal boundary-fitted grids. The code, which had been applied to a variety of engineering flow problems, included the possibility of non-staggered meshes (Rodi, Majumdar \& Schonung 1989).

\footnotetext{
${ }^{3}$ The TEACH code is the further development of the SIMPLE code by Caretto et al. (1972).

${ }^{4}$ Pressure-velocity coupling: The momentum equations are first solved as a predictor, using the pressure field found in the preceding iteration or time step. The divergence of the momentum equation leads to a Poisson equation for the pressure. Names such as SIMPLE, PISO, and SIMPLEC designate different versions of pressure-velocity couplings.

${ }^{5}$ The name hybrid is indicative of a combination of the central-difference and upwind scheme and can be considered as a three-line approximation.

${ }^{6}$ In the QUICK scheme, one adds one point in each direction and calculates the derivative using the cubic polynomial drawn through the four involved points. Local truncation error analysis shows third order accuracy.
} 

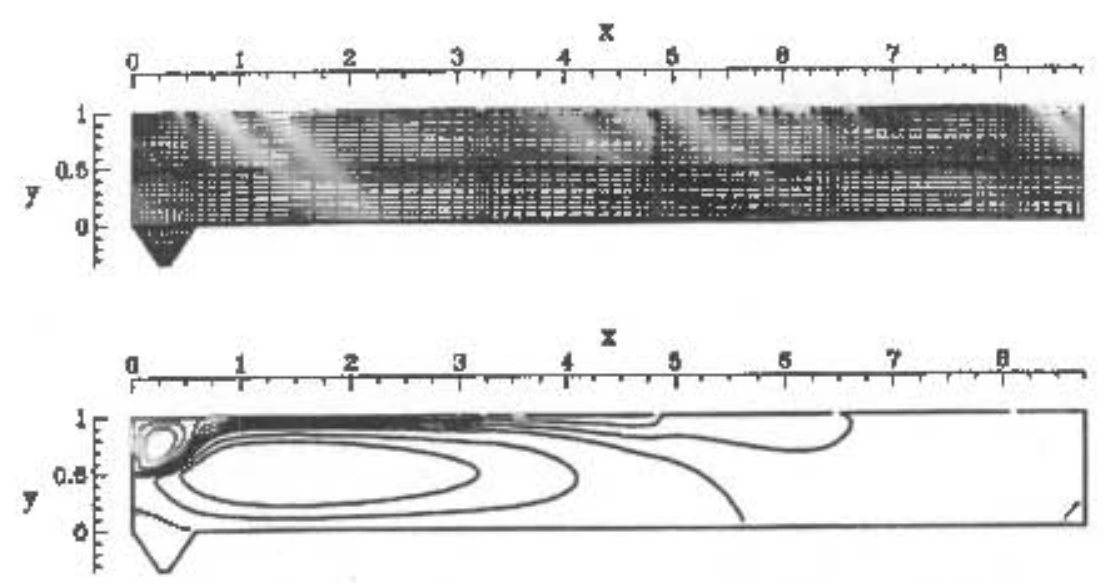

(a)

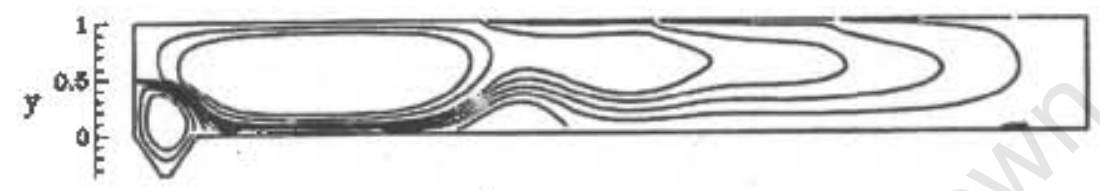

(b)

Figure 3.6: Numerical grid used for the computations; and comparison of streamline plot under two different model assumptions: (a) no eflects due to density differences and (b) effects of density differences included.

Zhou \& McCorquodale (1992a) made use of the settling function of Takścs et al. (1991), which led to improved modelling of the specific settling behaviour. The model was verified by application to three field invertigations of rectangular secondary clarifiers. The results showed that with the presence of a baffle the high entrainment of ambient fluid into the sinking influent plume coutd be reduced. The verified model was also nsed to study the performance of circular secondary clatifiers. The computational results illustrated that a skirt or reaction baflie had the same important function as in rectangular clarifiers. Zhou \& McCorquodale made use of a transient model to simulate the density variations with time in order to control the prevailing density variation process and to avoid divergence. The steady-state solution was then approached as a limit. The strong link botween the density and pressure fields produced fluctuating solutions if the change of the density source term was too rapid. Therefore, an underrelaxation of the density was adoptexl to slow down the changes in the derisity, which was also appliod to the density source term. In addition, a relatively low underrelaxation coefficient $^{7}$ was used to improve the iterative convergence of the Navier-Stokes

\footnotetext{
${ }^{7}$ The change in each variable is limited from one iteration to the next in order to prevent divergence. The optimal underselaxation factor is problem dependent.
} 
equations. In general they applied the SIMPLE algorithm in connection with a hybrid finite difference scheme.

McCorquodale \& Zhou (1993) investigated the effects of hydraulic and solids loading on clarifier performance. The result of the velocity field and concentration distribution in an idealized circular tank demonstrated that the flow pattern in a clarifier was more sensitive to changes in the densimetric Froude number, which reflects the ratio of inlet momentum to solids concentration, than to changes in the Reynolds number, which reflects the ratio of inlet momentum to viscosity effects.

Krebs (1991a,b) used the computer code PHOENICS (Rosten \& Spalding 1987) to compute steady two-phase flow, including the influence of buoyancy flow. Due to the well-known numerical instabilities of buoyancy-affected flow, turbulence was modelled by a constant turbulent viscosity that was 100 times higher than the laminar viscosity. Calculating velocity and volume fraction fields in a 2D rectangular model tank, Krebs illustrated qualitatively the effects of different geometric or hydraulic conditions. It was observed that a deep inflow could slow down the dominating density current at the bottom.

Based on new experiments on a different laboratory tank and a different numerical code, developed at the University of Karlsruhe in Germany, Krebs et al. $(1995,1996)$ investigated inlet and outlet arrangements. They pointed out that (i) the inlet should be positioned at the bottom of the tank to reduce potential energy, (ii) the kinetic energy could be dissipated with a series of angle bars beyond the inlet, and (iii) the inlet volume should be designed in such a way that turbulence will provide good flocculation conditions without causing floc break up. The effects of the outlet configurations had no significant influence on the bottom current; the position of the outlet, however, was decisive with respect to the deflection of the bottom current at the end wall.

The model used by Vitasovic et al. (1997) had its origin in the code of Zhou \& McCorquodale (1992a,b) and was verified against field data in Wahlberg et al. (1993). The close agreement between the observed measurements and the computational results validated the ability of the model to describe the operation of the clarifier. In further studies the influence of (i) the solids-settling characteristics, (ii) the effect of the surface overflow rate, and (iii) the significance of the clarifier geometry on the performance were analysed. The sludge blanket reacted 
sensitively to the settling parameter $n_{4}$ in the settling equation of Takács. High values of $n_{4}$ (or equivalently, high $S V I, D S V I$ or $\left.S S V I\right)^{8}$ increased the sludge blanket height and reduced the SS concentration of the return sludge. The eftuent SS concentration was directly correlated with the overflow rate and increased with increasing loading conditions. It was also found that small changes in the geometry of the tank could significantly improve the quality of the effluent. Reduction of the flocculent well from the existing $50 \%$ to $30 \%$ led to marked improvement under equivalent conditions of high flow.

SettlerCAD (Zhou et al. 1998), the dedicated 2D hydrodynamic SST simulation package, was developed by of Earth Tech (former Reith Crowther Consulting) and it follows the Vitasovic et al. code (1997). From an investigation to check whether or not 2D hydrodynamic models implicitly incorporate a reduction factor, which reduces the SST hydraulic and solids loading rate capacity below that of the 1D idealized flux theory, Ekama \& Marais (2002) found that SettlerCAD was mass conservative on underloaded conditions on the SST - the greater the overloaded conditions, the lower the sludge mass balance over the SST.

Lakehal et al. (1999) studied the sensitivity of the flow and concentration fields to parameters that characterized (i) the rheological properties of highly concentrated regions, (ii) the settling of sludge, and (iii) the effect of stratification on turbulent diffusion. They included the sludge blanket where the viscosity was affected by the rheological behaviour of the sludge. The test case was a circular, centre-feed secondary settling tank with sloped bottom and central withdrawal, and was performed on a nonorthogonal grid. The settling process was modelled using the double-exponential settling function of Takacs. In order to approximate the Bingham behaviour of activated sludge, model constants were extracted from the experiments in (Dick \& Ewing 1967 and Bolil \& Bewtra 1972). The simulations, in particular the sludge blanket height, reacted very sensitively to parameter or approach variations. Regions of high velocities and high gradients were strongly affected by these parameters. The following further observations were made:

1. use of the Bingham model resulted in a thicker sludge blanket; this could be increased further through an increase in the yield stress within the Bingham model. The results showed that the stiffer the sludge mixture behaved, the

\footnotetext{
${ }^{8}$ High Sludge Volume Indices $(S V T)$ indicate poor settling behaviour of the activated sludge.
} 
higher the developed sludge blanket.

2. The sludge blanket was also affected by a change in the settling function, while the peak velocity of the density current was hardly influenced. A better thickening characteristic of the sludge caused the sludge blanket to be more compact in height and to diminish faster with distance from the inlet.

3. In general, small eddy viscosity $\nu_{t}$ and low turbulent diffusive transport resulted in sharper velocity and concentration gradients throughout the tank, while high values of $\nu_{t}$ caused the sludge blanket to become thicker and to exhibit smoother concentration gradients at the interface to the clarified region, and also caused the main radial flow to decelerate and to have a generally upward trajectory.

Armbruster et al. (2000) used the mathematical model given in (Lakehal et al. 1999) to investigate the dynamic behaviour of a circular secondary clarifier based on a 24 hour measurement. Steady state simulations cannot model the sludge shift from the aeration tank induced by flow-rate increase and the transient phases causing sludge-blanket oscillations and potential sludge wash-out, and dynamic simulations are therefore important. The example of a dynamic simulation demonstrated that during the transient phase sludge wash-off occurred due to instabilities at the interface of the sludge blanket and supernatant. The measurements were carried out on a wastewater treatment plant in Germany. A stormwater situation was twice created by increasing the inflow while keeping the recycle flow rate nearly constant. Armbruster et al. pointed out that the deterioration of the effluent quality was more likely during and after the transient load increase or reduction phase than during high but steady-state loading.

\subsection{Closing comment}

Design procedures and numerical models of SSTs are based on empirical input. The empiricism comes in through the definition of the settling behaviour of activated sludge. These input parameters are often subject to calibration and are therefore strongly idealized. 
Empirical evidence indicates that sludge compaction limits the attainable underflow concentration (Ekama et al. 1997). Very little is known about this phenomenon, and due to the lack of adequate theoretical approaches, only poor estimates can be made concerning the recycle sludge concentration. With regard to the effluent suspended solids concentration, knowledge about the flocculation behaviour in the low concentration range is very important in order to enhance the incorporation of slow settling and nonsettleable particles into larger floc particles, which also improves the ESS concentration prediction.

Therefore carefully designed and planned experiments are needed in order to determine reliable parameters describing the two crucial sludge characteristics, flocculation and compaction, and to improve the efficiency of SSTs. Furthermore, the reliability of numerical models strongly depends on the quality of the empirical input.

In view of the algorithm and code presented in the thesis the following main areas of certainty can be extracted from the theoretical and computational research:

\section{The physical model:}

- the treatment of the two phases as a homogeneous fluid mixture with variable density

- the use of a concentration equation for the sludge involving the relative velocity

- the use of the Reynolds analogy of mass and momentum transport

- the use of either a constant turbulent viscosity or a variable turbulent viscosity predicted by the $k-\varepsilon$ model

- the difficulty in describing turbulent properties and quantities of the sludgewater mixture

- the need of further experiments for the improvement of the model.

\section{The numerical model:}

- the strong nonlinearity of sediment-induced flows in SSTs

- the strong link between density and pressure 
- the appearance of fluctuating solutions due to the high influence of the density term.

- the requirement to simulate the density variation with time in order to avoid divergence

- the need of underrelaxation for density and momentum

- the difficulty in simulating the density-waterfall in the inlet region

- numerical instabilities due to steep concentration gradients. 


\section{Chapter 4}

\section{Fundamental equations}

\subsection{Introduction}

Liquid-solid flows such as water and activated sludge consist of flows in which solid particles are carried by the liquid. These flows are classified as dispersed phase flows. Particle-particle and fluid-particle interaction makes the fluid mechanics of these flows complex. Nevertheless, these flows obey all of the basic laws of continuum mechanics; the equations are merely more complicated and more numerous than those of single-phase flows.

Generally, the flow of particles in fluids is a subcategory of multicomponent, multiphase flows. A component is a chemical species such as water and a phase is one of the states of matter, that is, gas, liquid, solid. Typical multicomponent single-phase flows are atmospheric flow and flows of emulsions. The flow of a liquid together with its vapour is an example of single-component multiphase flow, and slurries are an example of multicomponent multiphase flows.

Homogeneous liquid-solid flows such as emulsions consist of small particles, which are kept in suspension either by cohesive forces or by the turbulence of the carrier fluid. The homogeneous flows can be considered as a single-phase fluid with modified properties that depend on solids loading. The properties are weighted averages of velocity, density and transport properties (e.g. viscosity). Modelled in this way, the pseudofluid with averaged properties is assumed to obey the usual equations for motion of single-phase fluids, and the standard methods of fluid mechanics can be applied. 
Two methods, namely the Eulerian-Eulerian and the Eulerian-Lagrangian methods, have been extensively used to simulate particle dispersion, and the choice between these two is problem-dependent. The Eulerian methodology is mainly adopted for the prediction of interpenetrating media situations. This includes both highly particle loaded systems and dilute particle-laden flows. This approach can be employed in the one- and the two-fluid formulation.

In the first approach the particle concentrations are assumed to have some characteristics of a continuous phase. This method consists in solving an extra conservation law for the concentration of the particles or for their main spatial density. In order to consider buoyancy forces in the presence of a heavier dispersed phase, such as in the sedimentation process of SSTs, modifications of the transport equations are needed.

In the second approach the phases are treated as two interpenetrating continua. Each phase is governed by its own conservation and constitutive equations. The two phases are then coupled through interphase interaction properties. The two-phase approach is applied to mixtures of two immiscible fluids, such as air bubbles in water.

With the Lagrangian method individual particles are treated in a discrete way. The Lagrangian reference frame moves with the particles, and the instantaneous location of each particle is determined by reference to its origin and the time elapsed. Since tracking back individual particles relies on the properties of the flow field, the velocity and turbulence must be known. The Eulerian-Lagrangian formulation combines the two separate approaches. The Eulerian part delivers the flow field, and the Lagrangian part employs these data to track individual particles.

The Lagrangian formalism tracks the motion of the particles as they move through the flow field, which preserves their actual non-continuum behaviour. But with the Eulerian method the quantitative description of the variation in particle concentration is much easier since the Lagrangian methods require statistical sampling for the same purpose. The Lagrangian description may be difficult for flows with a large amount of particles, especially when the clouds of particles tracked is larger than the fluid parcel over which volume averaging is performed. Furthermore, with the Eulerian approach the phases can be computed over a single grid, whereas the Lagrangian description requires the 
interpolation of quantities between the local position of particles and the fixed grid nodes.

The two-phase flow in secondary settling tanks, consisting of water and activated sludge, can be regarded as an interpenetrating media situation. Furthermore, the dilute particle-laden flow in SSTs has the characteristics of a continuous phase, the so-called activated sludge.

The homogeneous theory of weighted averages cannot be applied to flows in secondary settling tanks. The differences in the density between the two phases, namely water and activated sludge, together with the low velocities in secondary settling tanks, lead to mutual momentum and mass transfer, and the vertical flow cannot be described by a suitable average velocity. Due to their higher density the suspended solids have a tendency towards sedimentation and to accumulation at the bottom of the tank, leading to a stratified flow field. The dynamics of sedimentation are determined by the interaction between buoyancy and drag forces. Equilibrium is reached when the net force, which acts on a solid, is zero and the particle is moving at its terminal settling velocity.

In order to take into account these phenomena the two phases, water and suspended solids (SS), are treated as a homogeneous fluid mixture with variable density. The local density of this fluid mixture depends on the local concentration of the particles. The distribution of the SS concentration is calculated by a particle mass conservation equation. This equation involves the relative velocity between the two phases due to their different densities. A density-state equation then gives the local density of the fluid mixture with respect to the local solids concentration. The coupling between the momentum equation and the solids transport equation/density state equation is achieved through the gravitydensity term in the momentum equation in the vertical direction (Figure 4.1).

The distribution of mean flow quantities in settling tanks is therefore governed by the conservation equations of mass, momentum and solid concentration. This physical model is mainly adopted by the researchers to predict the flow of SSTs (see chapter 3.2.2) and a summerary can also be found in Ekama et al. (1997). Gidaspow (1994) presents a comprehensive survey of the different physical models for describing multiphase flow. 
Modelling of Fluid-Solid Flow

in Secondary Settling Tanks

Fluid Mixture $=$ Pure Water + Suspended Solids

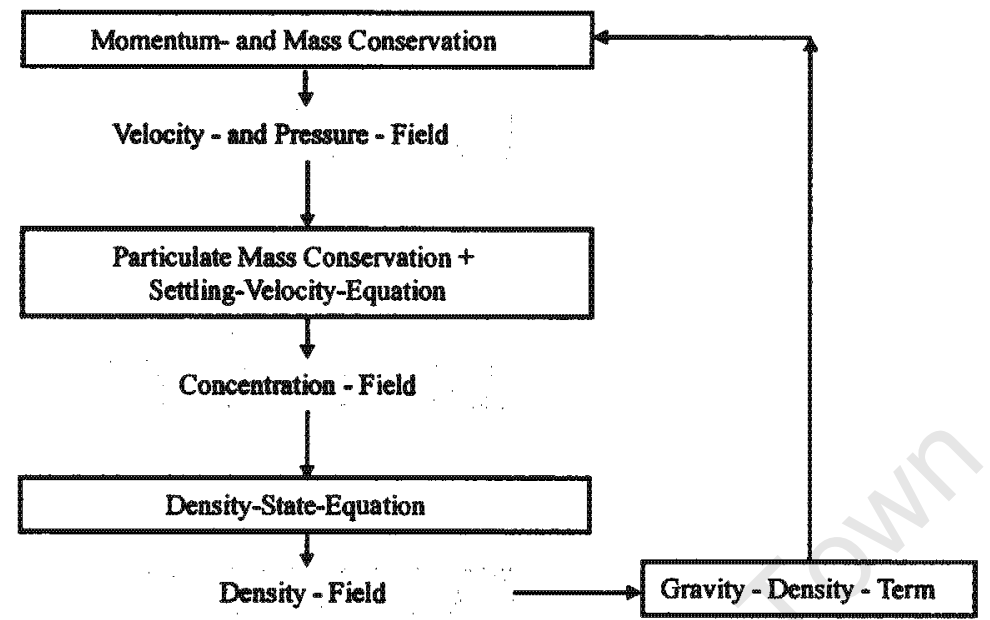

Figure 4.1: Modelling of fluid-solid flow in SSTs.

\subsubsection{Notation}

Before deriving the governing equations some details of notation are presented. Vectors and (second-order) tensors will be denoted in coordinate-free form by underscored symbols. Thus a vector $\underline{v}$ will have components $\left(v_{1}, v_{2}, v_{3}\right)$ relative to an orthonormal basis. In Cartesian and cylindrical polar coordinates we will write, respectively,

$$
\begin{aligned}
\underline{v} & =v_{z} \underline{e}_{x}+v_{y} \underline{e}_{y}+v_{z} \underline{e}_{z} \\
& =v_{r} \underline{e}_{r}+v_{y} \underline{e}_{y}+v_{\theta} \underline{e}_{\theta} .
\end{aligned}
$$

For plane and axisymmetric problems the components in the third direction, that is, with respect to $\underline{e}_{z}$ and $\underline{e}_{6}$, are omitted.

Similar remarks apply to tensors, so that a tensor $\underline{T}$ will have $N^{2}$ components $T_{i j}$ in $R^{N}$.

We employ the summation convention for repeated indices, so that, for example, $a_{i} b_{i}$ will be shorthand for $\sum_{i=1}^{N} a_{i} b_{i}$, for a problem in $R^{N}$.

It will be useful to write equations in a form that is applicable to both Cartesian and cylindrical coordinates, and for this purpose a parameter $m$ will be intro- 
duced, with the property that $m=1$ for cylindrical coordinates and $m=0$ for Cartesian coordinates (see Figure 4.2).

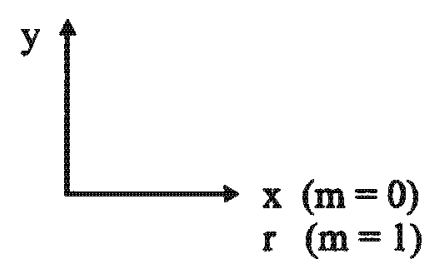

Figure 4.2: Coordinate systems for Cartesian and cylindrical coordinates.

\subsection{Conservation principles}

Conservation laws can be derived either by considering an arbitrary material volume or an arbitrary fixed control volume. A material volume enclosed by a surface has a fixed mass, invariant in time, and moves with the flow. The control volume is fixed in space and the fluid moves through it (Figure 4.3).
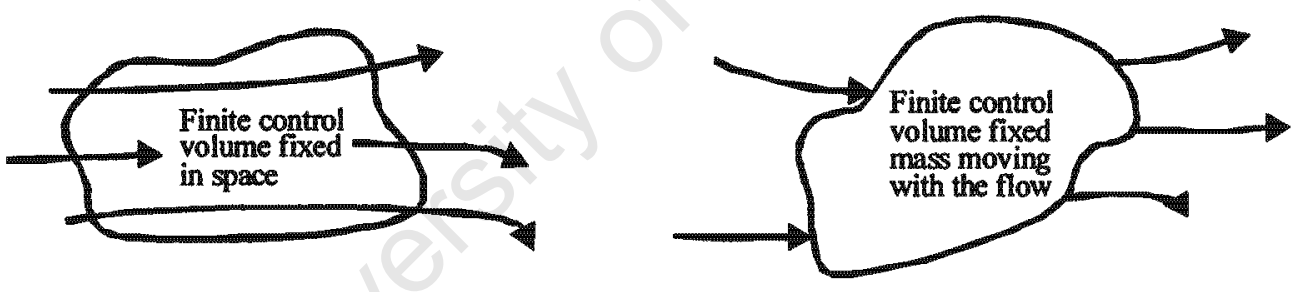

Figure 4.3: Control volume in space and control volume of fixed mass moving with the flow.

\subsubsection{Basic kinematics}

In a continuum, material points are assumed to be distributed continuously. In order to describe motion, a reference coordinate system at rest is needed, so that the motion of all particles can be referred with respect to it. A material point at a fixed reference time $t_{0}$ is denoted by $\underline{X}$ (Figure 4.4).

At time $t$ this point occupies a new position denoted by $\underline{x}$. A fundamental assumption is that there exists a smooth relation between the current position 


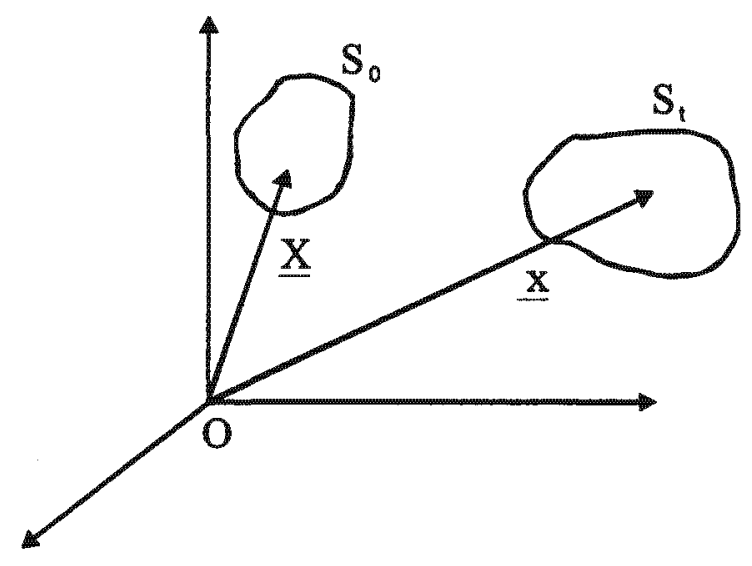

Figure 4.4: Motion of an arbitrary volume in a continuum.

of a material point relative to its reference position and time; that is,

$$
\underline{x}=\Phi(\underline{X}, t) .
$$

The function is required to be one-to-one and invertible, so that the reference position of a point can be obtained from its current position and the time:

$$
\underline{X}=\Phi^{-1}(\underline{x}, t) \text {. }
$$

It follows that descriptions of field quantities such as density, velocity, etc. can be given either as functions of $\underline{X}$ and $t$, or of $\underline{x}$ and $t$. The former is known as the material or Lagrangian description, and the latter is the spatial or Eulerian description. Problems in fluid mechanics are usually posed using the spatial description.

The function $\Phi$ must be at least first order differentiable in the spatial variables. It follows that, in order for the map to be invertible, and to exclude the possibility of inter-penetration of matter, the Jacobian, or deformation gradient, $\underline{F}$, defined by

$$
\underline{F}=\frac{\partial \Phi}{\partial X} \text { or } F_{i j}=\frac{\partial \Phi_{i}}{\partial X_{j}},
$$

must have a non-negative determinant; that is,

$$
J(\underline{X}, t)=\operatorname{det}\left(\frac{\partial x_{i}}{\partial X_{j}}\right)>0
$$

A physical property $\rho$ of the flow can be described by $\rho(\underline{X}, t)$ in the Lagrangian picture, and by $\hat{\rho}(\underline{x}, t)$ in the Eulerian picture. The material derivative of such a 
quantity is defined to be its rate of change with respect to time, for a material particle; that is,

$$
\frac{D \rho}{D t}=\left.\frac{\partial \rho}{\partial t}\right|_{\underline{\underline{X} \text { fixed }}} .
$$

For a given $\rho(\underline{X}, t)$ and the use of the chain rule we obtain

$$
\frac{D \rho}{D t}=\frac{\partial \rho}{\partial t}+\frac{\partial \rho}{\partial x_{i}} \frac{\partial x_{i}}{\partial t}=\frac{\partial \rho}{\partial t}+\frac{\partial \rho}{\partial x_{i}} u_{i}
$$

or

$$
\frac{D \rho}{D t}=\frac{\partial \rho}{\partial t}+\nabla \rho \cdot \underline{u}
$$

where $\underline{u}$ is the velocity.

The material derivative of a vector $\underline{w}$ is

$$
\frac{D w_{i}}{D t}=\frac{\partial w_{i}}{\partial t}+\frac{\partial w_{i}}{\partial x_{j}} \frac{\partial x_{j}}{\partial t}=\frac{\partial w_{i}}{\partial t}+\frac{\partial w_{i}}{\partial x_{j}} u_{j}
$$

or

$$
\frac{D \underline{w}}{D t}=\frac{\partial \underline{w}}{\partial t}+(\underline{u} \cdot \nabla) \underline{w} .
$$

\subsubsection{The Reynolds' transport theorem}

For any variable $\phi(\underline{x}, t)$ describing a property of the fluid, such as density, velocity, concentration, the integral over a moving material volume $V_{m}(t)$ enclosed by a surface $S(t)$ is

$$
\int_{V_{m}(t)} \phi(\underline{x}, t) d V
$$

The Reynolds' transport theorem states that the rate of change of this timedependent integral is given by

$$
\frac{D}{D t} \int_{V_{m}(t)} \phi(\underline{x}, t) d V=\int_{V_{c}} \frac{\partial \phi}{\partial t}+\int_{S_{c}} \phi(\underline{u} \cdot \underline{n}) d S_{c},
$$

where $V_{c}$ is the control volume fixed in space and the same as $V_{m}(t)$ at time $t, S_{c}$ is the boundary surface of $V_{c}, \underline{u}$ is the velocity and $\underline{n}$ is the unit outward normal vector (as shown in Figure 4.5).

Using the divergence theorem, we obtain 


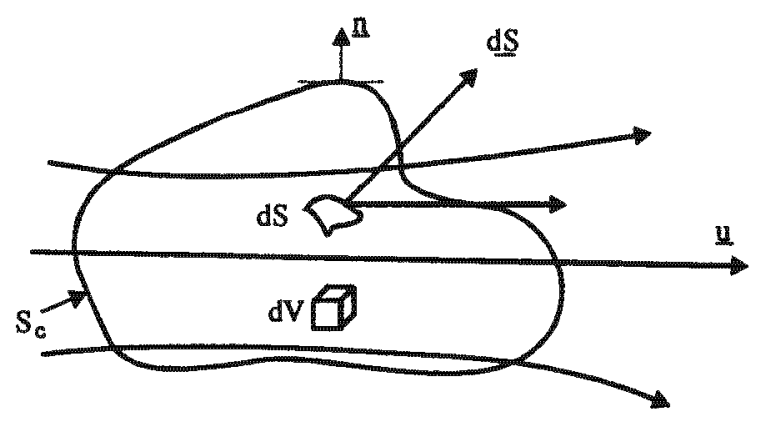

Figure 4.5: Finite control volume fixed in space.

$$
\begin{aligned}
\frac{D}{D t} \int_{V_{m}(t)} \phi(\underline{x}, t) d V & =\int_{V_{C}} \frac{\partial \phi}{\partial t} d V+\int_{V_{C}} \operatorname{div}(\phi \underline{u}) d V \\
& =\int_{V_{C}}\left[\frac{D \phi}{D t}+\phi \operatorname{div} \underline{u}\right] d V
\end{aligned}
$$

Reynolds' transport theorem states that the rate of change of the integral $\phi$ over a material volume $V_{m}(t)$ is equal to the volume integral of the rate of change of $\phi$ keeping $V_{m}(t)$ fixed, plus the surface integral of the rate of $\phi$ moving due to the surface velocity.

The conservation equations of mass, momentum and solid concentration will be derived in the following section.

\subsection{Conservation of mass: continuity equation}

The global principle of conservation of mass states that the total mass of a material volume is constant all times; that is,

$$
\frac{D}{D t} \int_{V_{m}(t)} \rho(\underline{x}, t) d V=0
$$

where $\rho$ is the mass density. Using Reynolds' Transport Theorem we obtain

$$
\int_{V_{C}} \frac{\partial \rho}{\partial t}=-\int_{S_{c}} \rho(\underline{u} \cdot \underline{n}) d S_{c}
$$

which states that the time rate at which mass is increasing inside a control volume is equal to the net rate of mass flow through the control surface. Using (4.12) 
and substituting $\rho$ for $\phi$ we obtain

$$
\int_{V_{C}}\left[\frac{D \rho}{D t}+\rho \operatorname{div} \underline{u}\right] d V=\int_{V_{C}}\left(\frac{\partial \rho}{\partial t}+\operatorname{div}(\rho \underline{u})\right) d V .
$$

Since $V_{c}$ is an arbritary control volume, we must have

$$
\frac{D \rho}{D t}+\rho \operatorname{div} \underline{u}=0,
$$

which is the continuity equation. Equivalently,

$$
\frac{\partial \rho}{\partial t}+\operatorname{div}(\rho \underline{u})=0 \text {. }
$$

\section{The continuity equations for flows in SSTs}

In this work the fluid in the SST is treated as a homogeneous liquid-solid continuum with variable density. The change of the fluid volume caused by the variable density of the fluid mixture is very small, so that the continuity equation for incompressible flow is assumed.

Using the notation described in Section 4.1 for cylindrical and Cartesian coordinates and introducing the parameter $m$, the continuity equation for twodimensional, incompressible flows in rectangular or circular settling tanks can then be written as

$$
\frac{\partial r^{m} u}{\partial r}+\frac{\partial r^{m} v}{\partial y}=0
$$

Here $u$ and $v$ are the velocity components in the $r$ (or $x$ ) and $y$ direction; $m=1$ yields the equation for cylindrical coordinates and $m=0$, with $r=x$, for Cartesian coordinates.

\subsection{Conservation of linear momentum: Navier- Stokes equations}

The physical principle is described by Cauchy's equation of motion, which states that the total force on a body equals its change of linear momentum. Forces acting on a fluid system may be classified as body forces, proportional to the volume or mass of the system, such as gravity, and surface forces, proportional to the area of surface on which they act, such as pressure and viscous forces. 


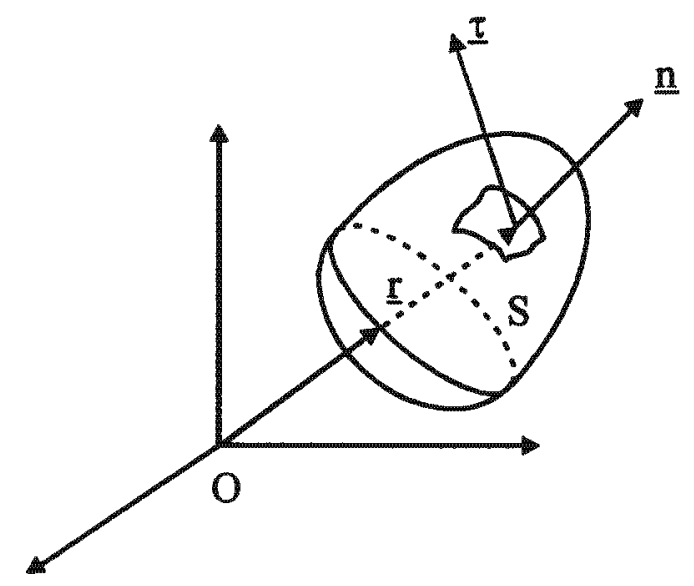

Figure 4.6: Arbitrary surface drawn in a tluid.

\subsubsection{Cauchy's stress principle}

When considering a volume $V$ enclosed by a surface that is embedded in a continuum, the effect of material outside of $V$ can be replaced by forces acting on $S$ with no change inside $V$. The totality of these forces gives rise to a field of surface forces. The force per unit area or traction $\tau$ acting on a surface $S$ with unit normal is defined as

$$
\tau(\underline{n})=\lim _{d S \rightarrow 0}\left(\frac{d \underline{F}}{d S}\right),
$$

where $E$ is the force acting across the surface element $d S$. The traction $\tau$ depends on the orientation of the surface element, defined through $\underline{n}$, as illustrated in Figure 4.6. Cauchy's theorem postulates that there exists a tensor $T$ such that

$$
\underline{\tau}=\underline{T n}
$$

or, in index form,

$$
\tau_{i}=T_{i j} n_{j}
$$

$T_{i j}$ is called the Cauchy stress tensor (Lai et al. 1993).

\subsubsection{Conservation of linear momentum}

Using equation with (4.11) the global principle of conservation of linear momentum becomes

$$
\frac{D}{D t} \int_{V_{m}(t)} \rho \underline{u}(\underline{x}, t) d V=\underline{E}
$$


The total force $\underline{F}$ comprises external (body) forces, such as gravity $g$, and surface forces, or tractions $\underline{\tau}$, such that

$$
\underline{F}=\int_{V} \rho \underline{g} d V+\int_{S} \underline{\tau} d S,
$$

where $\underline{g}=-g \underline{k}$ and $\underline{k}$ is the unit vector in the vertical upward direction. Since

$$
\int_{S} \underline{\tau} d S=\int_{S} \underline{T n} d S=\int_{V} \operatorname{div} \underline{T} d V
$$

where $\operatorname{div} \underline{T}$ is the vector with components $\partial T_{i j} / \partial x_{j}$, the local form of the conservation of linear momentum is

$$
\rho \frac{D \underline{u}}{D t}=\operatorname{div} \underline{\underline{T}}+\rho \underline{g} .
$$

It is called Cauchy's equation of motion.

From the conservation of angular momentum, $\underline{T}$ is symmetric: $T_{i j}=T_{j i}$.

In order to solve this equation the stress tensor $T$ must be specified in terms of other variables through a constitutive equation. This closure problem is described in the following subsection.

\subsubsection{Incompressible Newtonian fluid}

For an incompressible fluid, the stress takes the form

$$
\begin{aligned}
& \underline{T}=-p \underline{I}+\underline{\sigma} \quad \text { or } \\
& T_{i j}=-p \delta_{i j}+\sigma_{i j} \text { in component form, }
\end{aligned}
$$

where $-p \underline{I}$ is the isotropic part with the scalar $p$ being the general pressure, and $\underline{\sigma}$ is the extra stress. The tensor $\underline{\sigma}$ is a function of the deformation of the fluid.

A Newtonian fluid or linearly viscous fluid has the property that $\underline{\sigma}$ depends linearly on the rate of deformation tensor, with the dynamic viscosity coefficient $\mu$ as the proportionality constant. That is,

$$
\underline{T}=-p \underline{I}+2 \mu \underline{D},
$$

where the rate of deformation tensor $\underline{D}$ is defined by

$$
\underline{D}=\frac{1}{2}\left[(\nabla \underline{u})^{T}+\nabla \underline{u}\right] .
$$




\subsubsection{The Navier-Stokes equations for incompressible flows}

With the constitutive equation for an incompressible Newtonian fluid flow, we now obtain the equation of motion, called the Navier-Stokes equations for incompressible fluid flow. Substituting (4.28) in Cauchy's equation of motion (4.25) and setting div $\underline{u}=0$ for incompressible flows, we obtain

$$
\begin{aligned}
\rho \frac{D \underline{u}}{D t} & =\operatorname{div} \underline{T}+\rho \underline{g}=\operatorname{div}[-p \underline{I}+2 \mu \underline{D}]+\rho \underline{g} \\
& =-\nabla p+2 \mu \operatorname{div} \underline{D}+\rho \underline{g}=-\nabla p+\mu \operatorname{div}(\nabla \underline{u})+\rho \underline{g} .
\end{aligned}
$$

By using the definition of $D / D t$ given in (4.9) the acceleration $\underline{a}=D \underline{u} / D t$ can be written as

$$
\underline{a}=\frac{D \underline{u}}{D t}=\frac{\partial \underline{u}}{\partial t}+(\underline{u} \cdot \nabla) \underline{u},
$$

and the Navier-Stokes equations are

$$
\rho \frac{\partial \underline{u}}{\partial t}+\rho \underline{u}(\nabla \cdot \underline{u})=-\nabla p+\mu \operatorname{div}(\nabla \underline{u})+\rho \underline{g} .
$$

In the following subsection the Navier-Stokes equations for the special case of the two-dimensional, unsteady, flows in rectangular or circular settling tanks are derived.

\subsubsection{The Navier-Stokes equations for flows in SSTs}

For SSTs the body force is the gravitational body force $(-\rho g)$ in the vertical direction. The gravity term can be written as $\left[\rho_{r} g-\left(\rho-\rho_{r}\right) g\right]$, where $\rho_{r}$ is the reference density of clear water. Since only the gradient of the pressure is needed and the absolute value of the pressure is not important for incompressible flows, the first term $\rho_{r} g$ can be included with the pressure. Although the variations of the density $\rho$ are the cause of the particular fluid motion in SSTs, they are small and can be treated as constant in the unsteady and convection terms, while the density variation is retained only in the gravitational term. This is called the Boussinesq approximation.

Applying the Boussinesq approximation to the Navier-Stokes equations (4.32), we obtain, for two-dimensional, unsteady flows, 
conservation of momentum in the horizontal or radial direction $(x, r)$ :

$$
\begin{aligned}
\frac{\partial u}{\partial t}+u \frac{\partial u}{\partial r}+v \frac{\partial u}{\partial y}= & -\frac{1}{\rho} \frac{\partial p}{\partial r}+\frac{1}{r^{m}} \frac{\partial}{\partial r}\left(r^{m} \nu_{\text {eff }} \frac{\partial u}{\partial r}\right)+ \\
& \frac{1}{r^{m}} \frac{\partial}{\partial y}\left(r^{m} \nu_{\text {eff }} \frac{\partial u}{\partial y}\right)+S_{u}
\end{aligned}
$$

conservation of momentum in the vertical direction $(y)$ :

$$
\begin{aligned}
\frac{\partial v}{\partial t}+u \frac{\partial v}{\partial r}+v \frac{\partial v}{\partial y}= & -\frac{1}{\rho} \frac{\partial p}{\partial y}+\frac{1}{r^{m}} \frac{\partial}{\partial r}\left(r^{m} \nu_{\text {eff }} \frac{\partial v}{\partial r}\right)+ \\
& \frac{1}{r^{m}} \frac{\partial}{\partial y}\left(r^{m} \nu_{\text {eff }} \frac{\partial v}{\partial y}\right)+S_{v} .
\end{aligned}
$$

Here

$$
\begin{gathered}
m=\left\{\begin{array}{ll}
0 & \text { for Cartesian coordinates } \\
1 & \text { for cylindrical coordinates }
\end{array}\right\}, \\
S_{u}=\frac{1}{r^{m}} \frac{\partial}{\partial r}\left(r^{m} \nu_{\text {eff }} \frac{\partial u}{\partial r}\right)+\frac{1}{r^{m}} \frac{\partial}{\partial y}\left(r^{m} \nu_{\text {eff }} \frac{\partial v}{\partial r}\right)-2 \frac{\nu_{\text {eff }}}{r^{2}} u \cdot m
\end{gathered}
$$

and

$$
S_{v}=\frac{1}{r^{m}} \frac{\partial}{\partial r}\left(r^{m} \nu_{e f f} \frac{\partial u}{\partial y}\right)+\frac{1}{r^{m}} \frac{\partial}{\partial y}\left(r^{m} \nu_{e f f} \frac{\partial v}{\partial y}\right)-g \frac{\rho-\rho_{r}}{\rho} .
$$

The variables $u$ and $v$ are the velocity components in the $x$ (resp. $r$ ) and $y$ directions; $p$ is the general pressure less the hydrostatic pressure at reference density $\rho_{r} ;$ and $\rho$ is the fluid density. The last term in (4.36) represents the gravitydensity term. The effective viscosity $\nu_{\text {eff }}$ is the sum of the kinematic laminar and turbulent viscosity, which can be either a constant value or is obtained using a turbulence model, as described in Section 4.6.

\subsection{Conservation of particulate mass: the con- centration equation}

The equation for conservation of mass equation consists of a convective part that describes the particle motion, and a diffusive part that distributes the solids in the direction of the concentration gradient. It describes the balance of the temporal and spatial rates of change of solid mass and the solid mass transport movement due to mixing and settling. 
The integral form of the equation describing conservation of suspended solids concentration $c$, which is mass per mass, is analogous to the equations of mass and momentum conservation, and reads

$$
\frac{D}{D t} \int_{V_{m}(t)} c(\underline{x}, t) d V=A .
$$

Here $A$ includes diffusive transport of $c$ and a source term due to the settling motion of the suspended solids. Fick's law describes diffusion through the relation

$$
A_{d}=\operatorname{div}(\Gamma \nabla c),
$$

where $\Gamma$ is the diffusivity of the suspended solids. The effective diffusivity of the solid concentration $\Gamma=\nu_{e f f} / \sigma_{S}=\left(\nu_{l}+\nu_{t}\right) / \sigma_{S}$ is described with the use of the Reynolds analogy 1 of mass transport and momentum transport involving the $S c h m i d t$ number $\sigma_{S}$.

For the settling component we have

$$
A_{V_{S}}=\operatorname{div}\left(c V_{S}\right) \text {. }
$$

The coordinate-free form of (4.37) is then

$$
\frac{\partial c}{\partial t}+\underline{u} \cdot \nabla c=\operatorname{div}(\Gamma \nabla c)+\operatorname{div}\left(c V_{S}\right) .
$$

The two-dimensional, unsteady, turbulent (see Section 4.6), suspended solid concentration equation is therefore

$$
\begin{aligned}
\frac{\partial c}{\partial t}+u \frac{\partial c}{\partial r}+v \frac{\partial c}{\partial y}= & \frac{1}{r^{m}} \frac{\partial}{\partial r}\left(r^{m} \frac{\nu_{\text {eff }}}{\sigma_{S}} \frac{\partial c}{\partial r}\right)+\frac{1}{r^{m}} \frac{\partial}{\partial y}\left(r^{m} \frac{\nu_{\text {eff }}}{\sigma_{S}} \frac{\partial c}{\partial y}\right) \\
& +\frac{1}{r^{m}} \frac{\partial}{\partial y}\left(r^{m} V_{S} c\right) .
\end{aligned}
$$

\subsubsection{Solid settling equation}

As described in Section 2.4, various approaches have been made to extend the settling function $V_{S}$ to lower concentrations, so that the settling velocity decreases for decreasing concentration in the dilute region. Takács et al. (1991) used an

\footnotetext{
${ }^{1} \mathrm{O}$. Reynolds seems to be the first who discovered that the flow played an important role for mass and heat transfer. His analogy is besed on the discovery that mass and heat transfer depends on the momentum transfer in an analogous manner (Reynolds 1900).
} 
empirical approach to describe the settling velocity over the whole concentration range. The equation by Takács appears to be the best overall description of biological flocs in secondary clarifiers. The settling process is modelled using a double-exponential, which relates the solid settling velocity $V_{S}$ to the local concentration $c$ through

$$
V_{S}=V_{S 0} \cdot e^{-n_{4}\left(c-f_{n s} c\right)}-V_{S 0} \cdot e^{-n_{2}\left(c-f_{n e} c\right)} .
$$

Here $c$ is the suspended solids concentration, $f_{n s}$ is the non-settleable particle fraction, $n_{4}$ is an empirical coefficient for zone settling flocs, and $n_{2}$ is an empirical coefficient for the dilute settling particles.

Later the second part of (4.42) will be neglected, that is, the poorly settling particles are not taken into account. With that approximation (4.42) is identical to (4.43), which defines the settling velocity in the one-dimensional flux theory according to

$$
V_{S}=V_{S 0} \cdot e^{-n_{4} c} .
$$

Figure 4.7 shows the settling velocity against the concentration of the activated sludge for a set of parameters that is used for the simulation of the Darvill old and new SSTs, as well for the Watts SST (see Chapter 6). For example, with a feed concentration of $3.6 \mathrm{~g} / \mathrm{l}$ and a recycle concentration of $8.15 \mathrm{~g} / \mathrm{l}$ for Darvill Test 3 the settling velocity decreases by an order of magnitude for density from $0.47 \mathrm{~mm} / \mathrm{s}$ to $0.067 \mathrm{~mm} / \mathrm{s}$.

\subsubsection{Equation of state for density}

The state equation for density gives the relationship of density to concentration, through the linear equation

$$
\rho=\rho_{r}+c\left(1-\frac{\rho_{r}}{\rho_{S}}\right) .
$$

Here $\rho_{S}$ is the density of the dry particulate solids, $c$ is the solids concentration in the same units as $\rho_{S}$, and $\rho_{r}$ is the reference density (clear water). Figure 4.8 shows the density of the fluid mixture as a function of the solid concentration. Small changes in the density of the fluid mixture have large effects on the flow pattern in SSTs, as will be demonstrated in Chapter 6. For example, the liquid-solid density with a feed concentration of $3.6 \mathrm{~g} / \mathrm{l}$ is, with a density of $1000.83 \mathrm{~kg} / \mathrm{m}^{3}$, 
only $0.00083 \%$ higher than the reference density of clear water, and increases to $1001.88 \mathrm{~kg} / \mathrm{m}^{3}$ by a recycle sludge concentration of $8.15 \mathrm{~g} / \mathrm{l}$, which corresponds to less than $0.002 \%$ increase in the density of clear water.

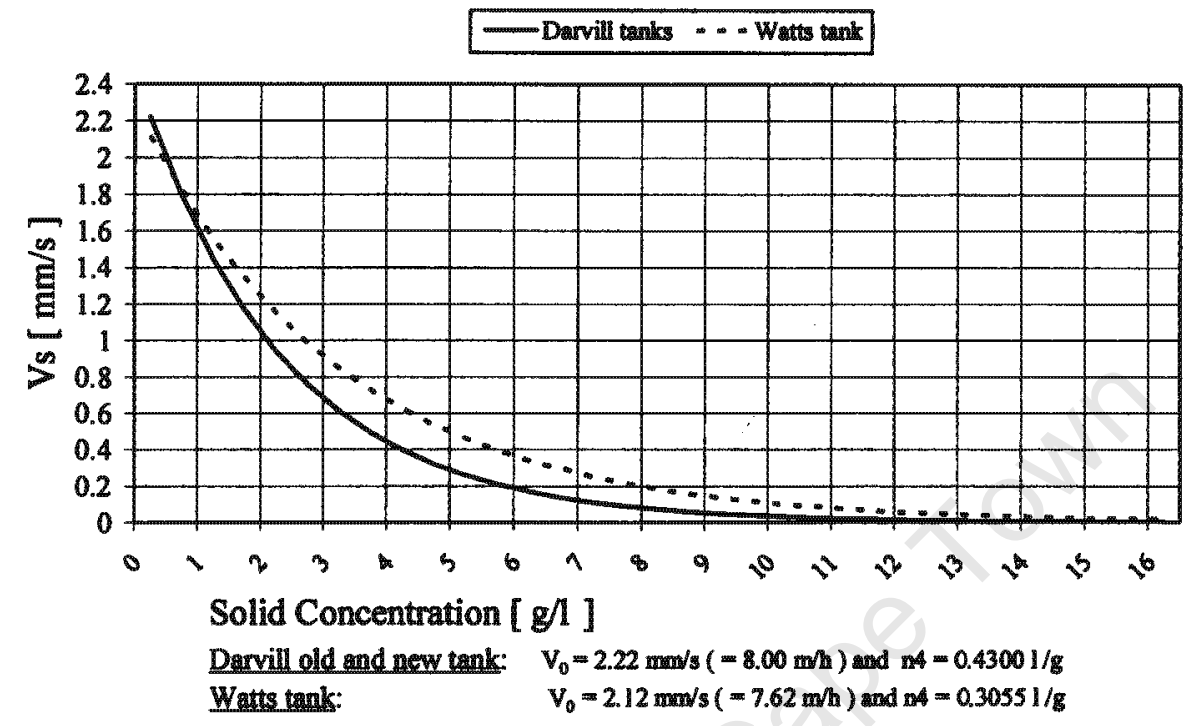

Figure 4.7: Modified settling function of Takács.

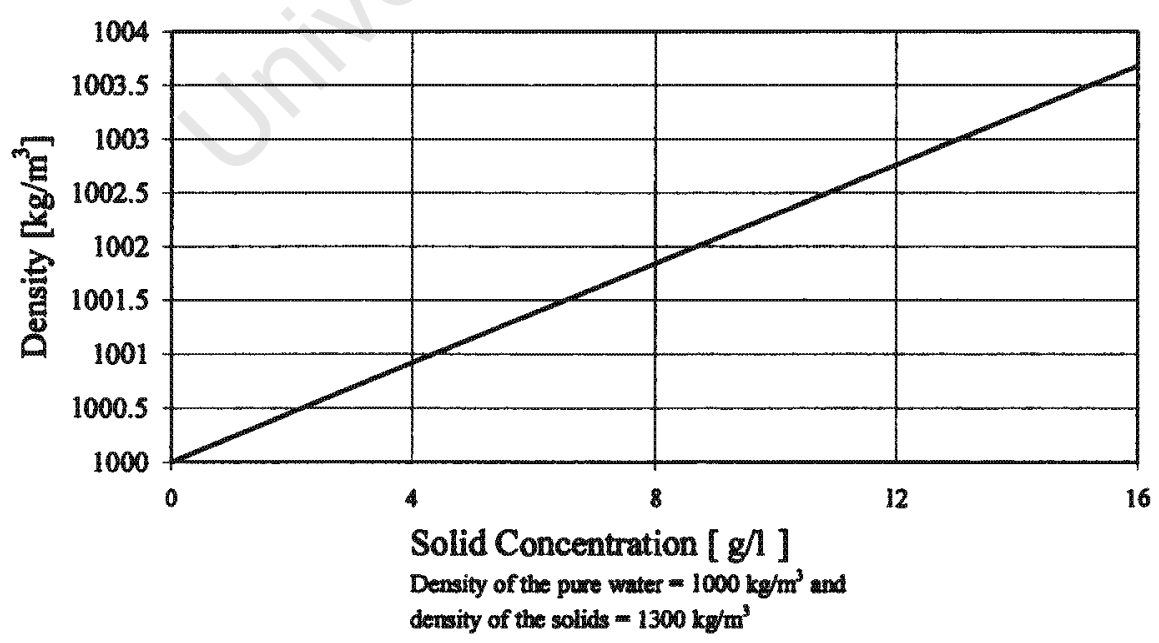

Figure 4.8: The state equation for density. 


\subsection{Turbulence}

\subsubsection{The nature of turbulence}

Flows of practical relevance are almost always turbulent. There is no unique definition of turbulent flow, but it has a number of characteristic features. In general turbulent fluid motion is irregular, random, unsteady and chaotic. Nevertheless, turbulence is deterministic and is described by the Navier-Stokes equations. Usually any variable in turbulent flow is divided into an averaged part $\bar{\phi}$, which is independent of time in steady flows, and a fluctuating part $\phi^{\prime}$, so that $\phi=\bar{\phi}+\phi^{\prime}$.

Turbulence can be characterized as an eddying motion, with a wide spectrum of eddy sizes. The eddies can be regarded as vortex elements, which stretch each other, thereby passing energy on to smaller and smaller eddies until viscous forces become active and dissipate the energy. The kinetic energy is extracted from the mean flow to the largest turbulent eddy and is transferred to the smallest eddy. This is called the energy cascade process (Figure 4.9). The width of the spectrum and the difference between the largest and the smallest eddies increase with the Reynolds number.

When buoyancy forces are present, there is also an exchange between potential energy of the mean flow and turbulent kinetic energy.

Turbulence is always three-dimensional. However, when the equations are time averaged the flow can be treated as two-dimensional.

\section{The cascade process}

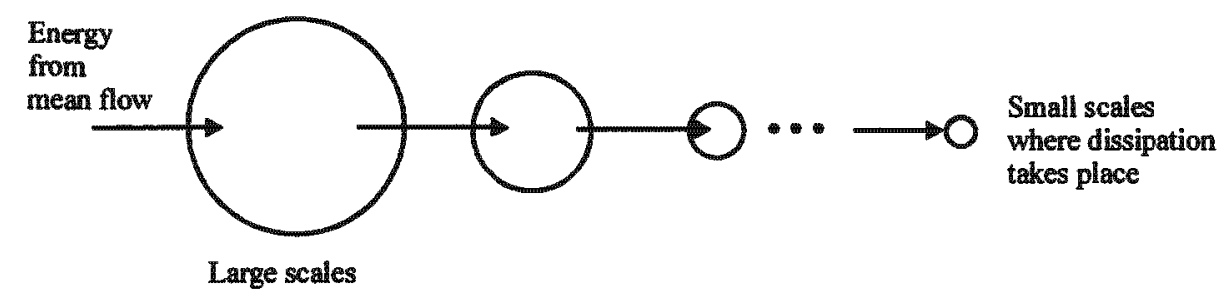

Figure 4.9: The energy cascade process. 


\subsubsection{Time-averaging procedure}

The most accurate approach to turbulence simulation is through solution of the Navier-Stokes equations. In such simulations, all of the motions contained in the flow are resolved. However, this would require a very fine resolution in space and time to resolve all turbulent scales. For flows of practical relevance the average character of real turbulence is of interest rather than the details of the fluctuating motion.

Different mathematical approaches have been adopted to describe turbulence. They differ in their complexity and their numerical expense. However, a turbulence model should simulate the average character of real turbulence and together with the mean flow equations it should form a closed set.

Generally, it is assumed that there are rapid and random fluctuations about the mean value (Figure 4.10). The largest eddies, which are of the order of the flow geometry, are treated as time-dependent, while the high frequency turbulence spectrum is modelled. The instantaneous values of the velocity, the pressure and scalar quantities such as as the sludge concentration, are decomposed into mean and fluctuating quantities, as follows:

$$
\begin{aligned}
\text { velocity } \underline{u}=\underline{\bar{u}}+\underline{u}^{\prime} \quad \text { with } \underline{\bar{u}}=\frac{1}{t_{2}-t_{1}} \int \underline{u} d t ; \\
\text { pressure } p=\bar{p}+p^{\prime} \quad \text { with } \bar{p}=\frac{1}{t_{2}-t_{1}} \int p d t \\
\text { concentration } c=\bar{c}+c^{\prime} \quad \text { with } \bar{c}=\frac{1}{t_{2}-t_{1}} \int c d t .
\end{aligned}
$$

Inserting these expressions into the continuity equation (4.16) and the momentum equation (4.32), as well as in the concentration equation (4.40), we obtain the time-averaged form of these equations, which in component form are

$$
\begin{gathered}
\bar{u}_{i, i}=0, \\
\frac{\partial \bar{u}_{i}}{\partial t}+\bar{u}_{j} \frac{\partial \bar{u}_{i}}{\partial x_{j}}=-\frac{1}{\rho} \frac{\partial \bar{p}}{\partial x_{i}}+\frac{\partial}{\partial x_{i}}\left[\nu\left(\frac{\partial \bar{u}_{j}}{\partial x_{i}}+\frac{\partial \bar{u}_{i}}{\partial x_{j}}\right)-\overline{u_{i}^{\prime} u_{j}^{\prime}}\right]-g \frac{\rho-\rho_{r}}{\rho} \delta_{i 2}, \\
\frac{\partial \bar{c}}{\partial t}+\bar{u}_{j} \frac{\partial \bar{c}}{\partial x_{j}}=\frac{\partial}{\partial x_{i}}\left[\frac{\nu}{\sigma_{S}} \frac{\partial \bar{c}}{\partial x_{i}}-\overline{u_{i}^{\prime} c}\right]+\frac{\partial\left(\bar{c} V_{S}\right)}{\partial x_{i}} \delta_{i 2} .
\end{gathered}
$$

The new equation set includes unknown correlations $\left(\overline{u_{i}^{\prime} u_{j}^{\prime}}\right.$ and $\left.\overline{u_{i}^{\prime} c}\right)$ between fluctuating velocities and between velocity and scalar fluctuations, due to the nonlinearity of the Navier-Stokes equations and the concentration equation. Physically, these correlations, multiplied by the density, represent the transport of 

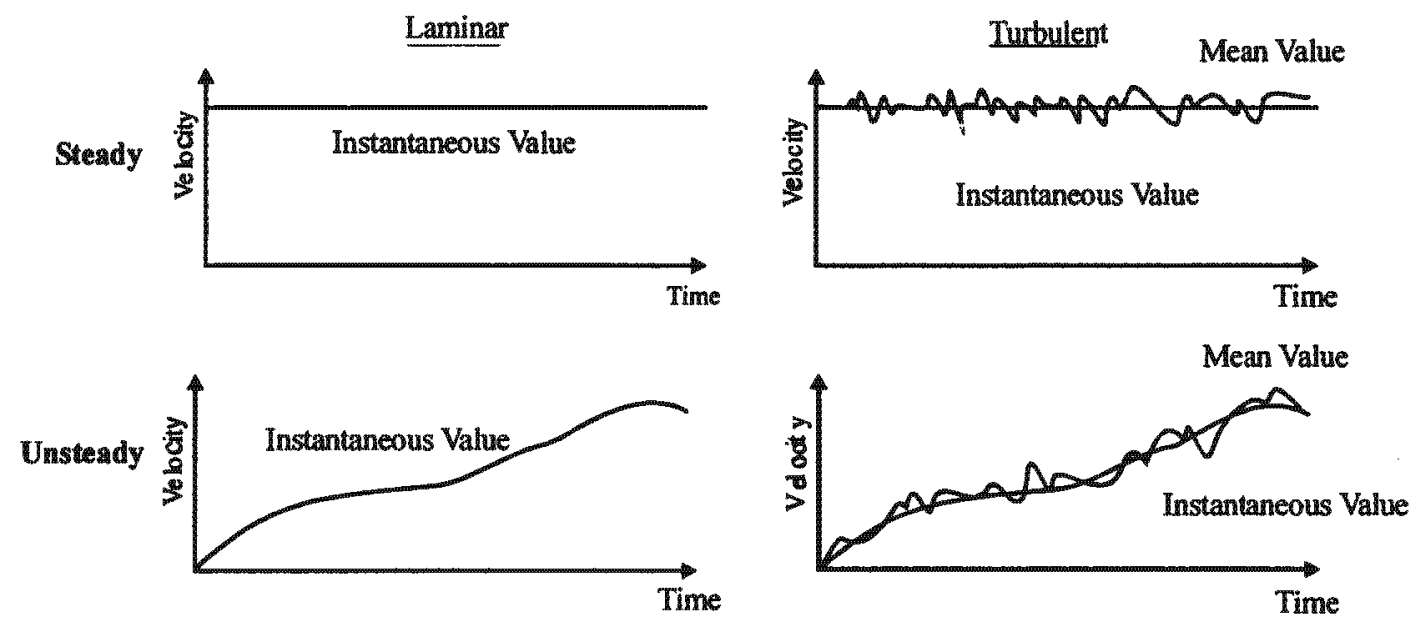

Figure 4.10: Laminar and turbulent velocity distribution for the steady and unsteady case.

momentum and mass transfer due to the fluctuating (turbulent) motion. These turbulent velocity fluctuations act as a stress on the fluid as a result of the increased internal friction. The correlation $\overline{u_{i}^{\prime} u_{j}^{\prime}}$ is therefore called the turbulent stress or Reynolds stress tensor in honour of Osborne Reynolds who introduced the time-averaging procedure in 1895. Likewise, the fluctuations of the concentration $\overline{u_{i}^{\prime} c}$ produce a turbulent mass flux. In most flow regions, the turbulent stresses and fluxes are much larger than their laminar counterparts.

Equations (4.48) to (4.50) are also exact since no assumption has been introduced in deriving them, however, they no longer form a closed set, that is, there are more unknowns than equations. A model for the turbulent quantities $\overline{u_{i}^{\prime} u_{j}^{\prime}}$ and $\overline{u_{i}^{\prime} c}$ is needed to close the system of equations.

There are different levels of approximations involved when closing the equation system. They differ in their complexity and their numerical cost. The most well known is the $k-\varepsilon$ model, which is used in this work, and which is described in Subsection 4.6.5. First, we introduce the concept of eddy viscosity.

\subsubsection{The concept of eddy viscosity}

Boussinesq (1897) proposed that the turbulent stresses are proportional to the mean-velocity gradients, by analogy with the viscous stresses in laminar flows; 
that is,

$$
-\overline{u_{i}^{\prime} u_{j}^{\prime}}=\nu_{t}\left(\frac{\partial \bar{u}_{j}}{\partial x_{i}}+\frac{\partial \bar{u}_{i}}{\partial x_{j}}\right)-\frac{2}{3} k \delta_{i j},
$$

where $k$ is the turbulent kinetic energy and $\nu_{t}$ is the turbulent or the so-called eddy viscosity. Instead of having only a laminar viscosity $\nu$, a turbulent viscosity $\nu_{t}$ is introduced, which depends strongly on the state of turbulence. The main problem is now shifted to determining the distribution of $\nu_{t}$. Boussinesq's proposal more than 100 years ago has turned out to become a significant part in almost all turbulence models of practical use.

From (4.51) we obtain the equation for $k$ by setting $i=j$, invoking the summation convention, and using the continuity equation. The kinetic energy $k$ of turbulent motion is then

$$
k=\frac{1}{2}\left(\overline{\left(u_{1}^{\prime}\right)^{2}}+\overline{\left(u_{2}^{\prime}\right)^{2}}+\overline{\left(u_{3}^{\prime}\right)^{2}}\right)=\frac{1}{2} \overline{u_{i}^{\prime} u_{i}^{\prime}}
$$

The eddy-viscosity concept is based on the assumption that the molecular motion in laminar flow is analogous to the turbulent motion. Likewise, in a fluid the turbulent eddies may be thought of as "particles" that collide and exchange momentum. Accordingly the eddy viscosity is assumed to be proportional to a velocity $\hat{V}$ characterizing the fluctuating motion, and to a typical length $\ell$ of this motion corresponding to the mean free path of the molecules in laminar flows; that is,

$$
\nu_{t} \propto \hat{V} \ell
$$

The well-known Prandtl mixing length hypothesis is based on this assumption (Prandtl 1925).

\subsubsection{The equation for turbulent energy $k$}

The averaged Navier-Stokes equations can be used to obtain a partial differential equation for the turbulent kinetic energy $k$ (4.49) by multiplying with $\bar{u}_{i}$ (Launder 1974, Rodi 1993, Wilcox 1993), and reads

$$
\frac{\partial k}{\partial t}+\overline{u_{j}} \frac{\partial k}{\partial x_{j}}=\underbrace{-\overline{u_{i}^{\prime} u_{j}^{\prime}} \frac{\partial \bar{u}_{i}}{\partial x_{j}}}_{\text {Production }}+\frac{\partial}{\partial x_{j}}\left[\nu \frac{\partial k}{\partial x_{j}}-\frac{1}{2} \overline{u_{i}^{\prime} u_{i}^{\prime} u_{j}^{\prime}}-\frac{1}{\rho} \overline{p^{\prime} u_{j}^{\prime}}\right]-\underbrace{\nu \overline{\frac{\partial u_{i}^{\prime}}{\partial x_{j}} \frac{\partial u_{i}^{\prime}}{\partial x_{j}}}}_{\text {三 Dissipation } \varepsilon} .
$$

The terms in (4.54) have the following meaning (see Rodi 1993, Wilfox 1993): 
- $\partial k / \partial t+u_{j} \partial k / \partial x_{j}=D k / D t$ is the rate of change of $k$ following a fluid particle;

- $-\overline{u_{i}^{\prime} u_{j}^{\prime}} \partial \overline{u_{i}} / \partial x_{j}$ is called the production and is the rate at which kinetic energy is transferred from the mean flow to the turbulence;

- the dissipation $\varepsilon$ is the rate at which $k$ is converted into thermal internal energy;

- $\partial / \partial x_{j}\left(\nu \partial k / \partial x_{j}\right)$ is the molecular diffusion of $k$;

- $\frac{1}{2} \overline{u_{i}^{\prime} u_{i}^{\prime} u_{j}^{\prime}}$ is called the turbulent transport and is the rate at which $k$ is transported through the fluid by turbulent fluctuations; and

- $\overline{p^{\prime} u_{j}^{\prime}} / \rho$ is called the pressure diffusion and is another form of turbulent transport.

Since the production term, turbulent diffusion terms, and dissipation terms are unknown, closure approximations have to be postulated for the new correlations. Invoking the Boussinesq eddy-viscosity approximation (4.51) and noting that the last term is zero for incompressible flow, we obtain, for the production term in (4.54),

$$
P_{k}=-\overline{u_{i}^{\prime} u_{j}^{\prime}} \frac{\partial \bar{u}_{i}}{\partial x_{j}}=\nu_{t}\left(\frac{\partial \bar{u}_{j}}{\partial x_{i}}+\frac{\partial \bar{u}_{i}}{\partial x_{j}}\right) \frac{\partial \overline{u_{i}}}{\partial x_{j}} .
$$

A gradient diffusion process is assumed for the turbulent transport and pressure diffusion, which diffuses $k$ from region of high $k$ to those of small $k$. We get

$$
\frac{1}{2} \overline{u_{i}^{\prime} u_{i}^{\prime} u_{j}^{\prime}}+\frac{1}{\rho} \overline{p^{\prime} u_{j}^{\prime}}=-\frac{\nu_{t}}{\sigma_{k}} \frac{\partial k}{\partial x_{j}},
$$

where $\sigma_{k}$ is an empirical diffusion coefficient.

In turbulent flows kinetic energy is dissipated by frictional forces (viscous stresses) and transformed into internal energy. As a result of the cascade process, kinetic energy dissipated at the small scales can be estimated using the large scale numbers $\hat{V}$ and $\ell$. The dissipation $\varepsilon$, the energy per unit time and unit mass $\left(\varepsilon=\left[\mathrm{m}^{2} / \mathrm{s}^{3}\right]\right)$, depends on the viscosity $\nu$ and the fluctuating velocity gradients. Using dimensional analysis and considering that the velocity scale is now $\hat{V}=\sqrt{k}$, the expression

$$
\varepsilon \equiv \nu \overline{\frac{\partial u_{i}^{\prime}}{\partial x_{j}} \frac{\partial u_{i}^{\prime}}{\partial x_{j}}}=c_{D} \frac{k^{\frac{3}{2}}}{\ell}
$$


for $\varepsilon$ is obtained, where $c_{D}$ is an empirical constant.

Incorporating these terms into (4.54) the equation for $k$ now reads

$$
\frac{\partial k}{\partial t}+u_{j} \frac{\partial k}{\partial x_{j}}=P_{k}+\frac{\partial}{\partial x_{j}}\left(\nu+\frac{\nu_{t}}{\sigma_{k}} \frac{\partial k}{\partial x_{j}}\right)-c_{D} \frac{k^{\frac{3}{2}}}{\ell} .
$$

In order to complete the equation Kolmogorov and Prandtl introduced independently the eddy viscosity relation

$$
\nu_{t}=c_{\mu}^{\prime} \sqrt{k} \ell
$$

which is known as the Kolmogorov-Prandtl expression (Kolmogorov 1942, Prandtl 1945).

Most single-equation models of turbulence, such as (4.58) with (4.59), do not offer much improvement over algebraic models. In order to improve these turbulence models a second equation is postulated from which the turbulence length scale can be computed. The most popular one is the $k-\varepsilon$ model, in which the dissipation $\varepsilon$ is obtained from its own transport equation, and the dissipation term $c_{D} k^{\frac{3}{2}} / \ell$ is simply $\varepsilon$. The $k-\varepsilon$ model will be described in the following subsection.

\subsubsection{The $k-\varepsilon$ turbulence model}

Although an exact equation for the dissipation can be derived from the NavierStokes equation (Wilcox, 1993), the very large number of unknown correlations makes it impractical. It is therefore better to derive an equation for $\varepsilon$ based on its physical meaning. In the exact equation for $\varepsilon$ the production term includes, as in the $k$ equation, mean flow gradients and turbulent quantities, and the dissipation term includes only turbulent quantities. Thus, deriving the modelled $\varepsilon$ equation from the modelled $k$ equation, the right-hand side can be written as

$$
\frac{\partial}{\partial x_{j}}\left(\nu+\frac{\nu_{t}}{\sigma_{\varepsilon}} \frac{\partial \varepsilon}{\partial x_{j}}\right)+\frac{1}{t}\left(c_{\varepsilon 1} P_{k}-c_{\varepsilon 2} \varepsilon\right) \text {. }
$$

Two additional constants have been added for the source terms, that is, the production term and the dissipation term. In order to make sure that the correct dimensions are recovered, a turbulent time scale $t=k / \varepsilon[s]$ has been added. We can therefore write the equation for $\varepsilon$ as

$$
\frac{\partial \varepsilon}{\partial t}+u_{j} \frac{\partial \varepsilon}{\partial x_{j}}=\frac{\partial}{\partial x_{j}}\left(\nu+\frac{\nu_{t}}{\sigma_{\varepsilon}} \frac{\partial \varepsilon}{\partial x_{j}}\right)+\frac{k}{\varepsilon}\left(c_{\varepsilon 1} P_{k}-c_{\varepsilon 2} \varepsilon\right) .
$$




\begin{tabular}{|l|l|l|l|l|}
\hline$c_{\mu}$ & $c_{\varepsilon 1}$ & $c_{\varepsilon 2}$ & $\sigma_{k}$ & $\sigma_{\varepsilon}$ \\
\hline 0.09 & 1.44 & 1.92 & 1.0 & 1.3 \\
\hline
\end{tabular}

Table 4.1: Values of the constants in the $k-\varepsilon$ model

Combining equations (4.60) and (4.61) we obtain the expression

$$
\nu_{t}=c_{\mu} \frac{k^{2}}{\varepsilon}
$$

for the eddy viscosity $\nu_{t}$, with $c_{\mu}=c_{\mu}^{\prime} c_{D}$.

Equations (4.60), (4.61) and (4.62) form the $k-\varepsilon$ model, which includes five universal constants. A detailed description of the derivation of standard values for $c_{\mu}, c_{\varepsilon 1}, c_{\varepsilon 2}, \sigma_{k}$ and $\sigma_{\varepsilon}$ can be found in (Rodi 1993). The standard values of the five constants are listed in Table 4.1.

\subsubsection{The universal log-law of the wall}

Turbulent flows near solid walls are anisotropic and require special attention. Due to the no-slip condition, both mean and fluctuating velocities are zero, while the dissipation $\varepsilon$ is finite. Very close to the wall viscous stresses dominate over turbulent stresses. This region is called the viscous sublayer region. Away from the wall turbulent motion increases and turbulent stresses dominate over viscous stresses. For the theory it is assumed that close to the wall the total stress is constant, and we therefore have

$$
\tau_{w}=\mu \frac{d \bar{u}}{d y}-\rho \overline{u^{\prime} v^{\prime}}
$$

where $\mu$ is the laminar dynamic viscosity, $\bar{u}$ is the averaged velocity parallel to the wall, $y$ is the normal distance to the wall, and $\overline{\rho u^{\prime} v^{\prime}}$, the turbulent shear stress, as defined above with $\rho$ as the density. The averaged velocity $\bar{u}$ depends now only on $y$ and is completely described by $\tau_{w}, \rho$ and $\mu$. Thus, this correlation can be described in dimensionless form. For simplicity the friction velocity $u_{\tau} \equiv \sqrt{\tau_{w} / \rho}$ is defined, and the velocity distribution can now be expressed by

$$
\frac{\bar{u}}{u_{\tau}}=f\left(y \frac{\rho u_{\tau}}{\mu}\right)=f\left(y \frac{u_{\tau}}{\nu}\right) \text {. }
$$

In the laminar sublayer we have $f\left(y u_{\tau} / \nu\right)=y u_{\tau} / \nu$, and for larger distances to the wall the turbulent motion gets closer to the fully developed state, which is 
independent of $\mu$, that is, $-\overline{u^{\prime} v^{\prime}} \approx u_{\tau}^{2}$. The motion at the wall is governed by the sizes $u_{r}$ and $y$, therefore the dimensionless equation for $d \bar{u} / d y$ is

$$
\frac{d \bar{u}}{d y}=\frac{1}{\kappa} \frac{u_{\tau}}{y}
$$

where $\kappa$ is the Karmán constant, which is a fundamental and universal constant of this kind of turbulence problem and is obtained through experiments to $\kappa \approx 0.4$. The integration of (4.65) yields

$$
\bar{u}=\frac{u_{\tau}}{\kappa} \ln y+C^{*},
$$

or

$$
u^{+}=f\left(y \frac{u_{\tau}}{\nu}\right)=\frac{1}{\kappa} \ln y \frac{u_{\tau}}{\nu}+C .
$$

In the sublayer region the function $f\left(y \frac{u_{\tau}}{\nu}\right)$ is linear, while in the turbulent core it is logarithmic.

With measurements on pipe flows Nikuradse (1932) found that the velocity distribution for smooth pipes is always given by

$$
\frac{\bar{u}}{u_{\tau}}=\frac{1}{0.4} \ln \left(\frac{y u_{\tau}}{\nu}\right)+5.5, \text { independent of the Reynolds number. }
$$

This expression is called the universal log-law of the wall due to its validity for all Reynolds number of the mean flow. The velocity profile in a boundary layer shows three main regions:

- the viscous sublayer: $y^{+} \lesssim 5$ where $u^{+}=y^{+}$,

- the turbulent core region: $30 \lesssim y^{+}<100$,

- the wake region: above the logarithmic region $\left(y^{+} \geq 100\right)$.

The buffered region $5 \lesssim y^{+}<30$ is an intermediate region between the viscous sublayer and the logarithmic region, where neither the linear law nor the log law are valid.

\subsubsection{Wall functions}

In order to predict the sharp gradients prevailing at wall boundaries, the grid has to be sufficiently fine, which often requires too great computer resources. 
Especially at high Reynolds number, the viscous sublayer is so thin that it is difficult to construct a mesh fine enough. Due to these difficulties it is common practice to prescribe boundary conditions employing wall functions. With the assumption that the flow near the wall behaves like a fully developed turbulent boundary layer, the use of the law of the wall and the logarithmic law of the wall can be made.

In the viscous sublayer with $u^{+}=y^{+}$the law of the wall represents the wall shear stress according to

$$
\tau_{w}=\mu \frac{\overline{u_{t}}}{y_{n}},
$$

where $u_{t}$ is the tangential velocity and $y_{n}$ is the normal distance to the solid boundary.

The shear stress in the turbulent core region can be derived from equation (4.67) using the expression for $y^{+}=y u_{\tau} / \nu$, as follows:

$$
\tau_{w}=\mu \frac{\kappa y^{+}}{\ln \left(E y^{+}\right)} \frac{\overline{u_{t}}}{y_{n}},
$$

where $E=9$ for smooth walls (Rodi 1993). Equation (4.70) implies that the required turbulent viscosity will have the form

$$
\mu_{w}=\mu \frac{\kappa y^{+}}{\ln \left(E y^{+}\right)} .
$$

The appropriate wall function relations can be obtained in a recursive manner.

In a fully turbulent boundary layer with $30 \lesssim y^{+}<100$ the production term and the dissipation term are much larger than the other terms. With this nearwall assumption that production equals dissipation $\left(0=P_{k}-\varepsilon\right)$, the boundary conditions for $k$ and $\varepsilon$ are derived in terms of the wall shear stress.

The Boussinesq assumption for the shear stress (4.51) reads $\tau_{w}=-\rho \overline{u^{\prime} v^{\prime}}=$ $\rho \nu_{t}(\partial \bar{u} / \partial y)^{2}$ and with $\nu_{t}=k^{2} / \varepsilon(4.62)$ the boundary condition of the turbulent kinetic energy is

$$
k_{n}=\frac{\left(\tau_{w} / \rho\right)^{2}}{\left(c_{\mu}\right)^{0.5}} .
$$

The dissipation $\varepsilon$ is in balance with the production $P_{k}$, the boundary condition of $\varepsilon$ can be written as

$$
\varepsilon_{n}=\frac{\left(\tau_{w} / \rho\right)^{3}}{\kappa y_{n}},
$$


where the velocity gradient in the production term $-\overline{u^{\prime} v^{\prime}} \partial \bar{u} / \partial y$ has been computed from the log-law (4.68), that is, $\partial \bar{u} / \partial y=u_{\tau} / \kappa y_{n}$.

The values for $k_{n}$ and $\varepsilon_{n}$ are applied at the near-wall node as boundary conditions (Hill \& Baskharone 1997).

\subsubsection{The $k-\varepsilon$ equations for SSTs}

Using the above notation for rectangular or circular settling tanks, the twodimensional $k-\varepsilon$ equations can be written in the form

$$
\begin{aligned}
\frac{\partial k}{\partial t}+u \frac{\partial k}{\partial r}+v \frac{\partial k}{\partial y}= & \frac{1}{r^{m}} \frac{\partial}{\partial r}\left(r^{m} \frac{\nu_{t}}{\sigma_{k}} \frac{\partial k}{\partial r}\right)+\frac{1}{r^{m}} \frac{\partial}{\partial y}\left(r^{m} \frac{\nu_{t}}{\sigma_{k}} \frac{\partial k}{\partial y}\right) \\
& +P_{1}+P_{2}-\varepsilon
\end{aligned}
$$

and

$$
\begin{aligned}
\frac{\partial \varepsilon}{\partial t}+u \frac{\partial \varepsilon}{\partial r}+v \frac{\partial \varepsilon}{\partial y}= & \frac{1}{r^{m}} \frac{\partial}{\partial r}\left(r^{m} \frac{\nu_{t}}{\sigma_{\varepsilon}} \frac{\partial \varepsilon}{\partial r}\right)+\frac{1}{r^{m}} \frac{\partial}{\partial y}\left(r^{m} \frac{\nu_{t}}{\sigma_{\varepsilon}} \frac{\partial \varepsilon}{\partial y}\right) \\
& +c_{1} \frac{\varepsilon}{k} P_{1}-c_{2} \frac{\varepsilon^{2}}{k},
\end{aligned}
$$

where

$$
P_{1}=2 \nu_{t}\left[\left(\frac{\partial u}{\partial r}\right)^{2}+\left(\frac{\partial v}{\partial y}\right)^{2}+\left(\frac{u}{r}\right)^{2} m+\frac{1}{2}\left(\frac{\partial u}{\partial y}+\frac{\partial v}{\partial r}\right)^{2}\right]
$$

represents the rate of production of turbulent kinetic energy resulting from the interaction of the turbulent stresses and velocity gradients, and

$$
P_{2}=\frac{g}{\rho_{r}} \frac{\nu_{t}}{\sigma_{c}} \frac{\partial\left(\rho-\rho_{r}\right)}{\partial y}
$$

the rate of production due to buoyancy effects.

Generally, the turbulent transport is strongly affected by buoyancy effects and the buoyancy term $P_{2}$ represents an exchange between the turbulent kinetic energy and potential energy. In stable stratification of SSTs the term $P_{2}$ becomes negative and represents a sink term. Turbulent viscosity and turbulent diffusivity are reduced and the turbulence is damped while the potential energy of the system increases. In the case of unstable stratification, in which fluid of higher density lies above fluid of lower density, turbulent energy is produced at the expense of potential energy. 


\section{Chapter 5}

\section{Numerical modelling}

\subsection{Introduction}

The numerical model used in this work is based on the finite element method (FEM), which is a method for obtaining approximate solutions to partial differential equations. It is widely used especially in problems arising in physics and engineering. The key feature of the method lies in the subdivision of the spatial domain into a finite number of subdomains or elements, typically triangles or quadrilaterals in two dimensions, and tetrahedra or hexahedra in three. Over each finite element, the unknown variables (e.g. velocity, concentration etc.) are approximated using simple functions such as polynomials and the problem is reduced in this way to one of solving a set of simultaneous ordinary differential equations (ODEs) in time. In the case of steady problems, one obtains a set of simultaneous algebraic equations, while for time-dependent problems, discretization in time is required to reduce the set to ODEs to algebraic equations. The FEM is a powerful tool because of its ability to model problems in domains that are geometrically complex.

The FEM has been in existence since the mid 1950s, when efforts were made to describe the overall behaviour of simple elastic bars through the use of small, discrete elements (Newmark, 1949). The term 'finite element' was first used in the paper of Clough (1960). The versatility of the method and its underlying rich mathematical basis made it applicable to a wide range of problems. For example, Zienkiewicz and Chung (1965) applied the method to field problems, such as heat 
conduction and irrotational flow, the solution of Laplace and Poisson equations, and Oden (1972) was among the first who worked on nonlinear problems. Since then the FEM became one of the most important developments in computational methods to occur in the last century, and numerous articles and texts have been published, and new applications appear routinely in the literature. The handbook by Kardestuncer (1987), for example, presents a comprehensive survey of finite element theory and practice available at that time. Other reviews and descriptions of the method can be found in the texts by Fletcher (1984), Reddy (1984), Hughes (1987), and Zienkiewicz \& Taylor (1989, 2000), and programming aspects are described for example by Taylor \& Hughes (1981), Lucquin \& Pironneau (1998), Smith \& Griffiths (1997), or Burnett (1987). The book of B.D. Reddy (1998) gives a very good and comprehensive introduction to the mathematical analysis of the finite element method.

Historically the Rayleigh-Ritz method has played a significant role in the development of the finite element method (Rayleigh 1877, Ritz 1909). The method uses the formulation of the problem in variational or miminization form, and approximates the solution in a finite-dimensional space. This leads to the much simpler problem of solving a system of simultaneous algebraic equations, for steady problems.

The Rayleigh-Ritz method was used extensively throughout the 1960s in applications to problems in solid mechanics. However, the method cannot be extended to certain classes of problems such as those for Newtonian fluids (the NavierStokes equations), which do not have a minimization principle. This deficiency can be overcome through the use of the method of weighted residuals, and in particular the Galerkin method, devised by Galerkin in 1915. In this method an integral formulation of the problem can be constructed without resort to a minimization problem, and the search for an approximate solution in a finitedimensional space again leads to the solution of a system of ODEs, or of algebraic equations if the problem is time-dependent.

\subsection{The method of weighted residuals}

As derived and described in Chapter 4, continuum problems that arise in engineering and physics are usually described by systems of partial differential 


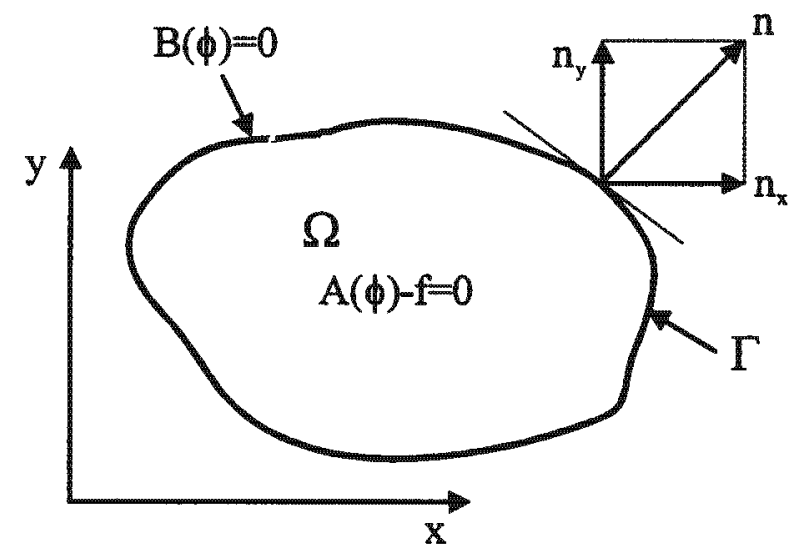

Figure 5.1: Problem domain $\Omega$ and boundary $\Gamma$.

equations. These initial boundary value problems can be written in the general form

$$
\mathbf{A}(\phi)-\mathbf{f}=\left\{\begin{array}{c}
A_{1}(\phi) \\
A_{2}(\phi) \\
\vdots
\end{array}\right\}-\left\{\begin{array}{c}
f_{1} \\
f_{2} \\
\vdots
\end{array}\right\}=0
$$

together with certain boundary conditions

$$
\mathbf{B}(\phi)=\left\{\begin{array}{c}
B_{1}(\phi) \\
B_{2}(\phi) \\
\vdots
\end{array}\right\}=\left\{\begin{array}{c}
g_{1} \\
g_{2} \\
\vdots
\end{array}\right\}
$$

where $\phi$ is the unknown set of functions, $A_{1}, A_{2}, \ldots$ are partial differential operators in space and time defined on the domain $\Omega, \mathbf{f}$ and $\mathbf{g}$ are given vectors, and $B$ is a set of boundary conditions on the boundary $\Gamma$ (see Figure 5.1). If the problem is time-dependent, it requires also a set of initial conditions on $\phi$.

As the set of equations (5.1) has to be zero at each point of the domain $\Omega$, the residual $\mathbf{R}$ of any function $\widetilde{\phi}$ defined by

$$
\mathbf{R}(\tilde{\phi})=\mathbf{A}(\tilde{\phi})-\mathbf{f}
$$

vanishes when $\widetilde{\phi}=\phi$. It is in general not possible to find an analytical solution that satisfies both the differential equation and the boundary and initial conditions. The weighted residual method involves searching for a solution of $\widetilde{\phi}$ that minimizes the residual in some sense. In this method $\mathbf{R}(\widetilde{\phi})$ is multiplied 
by a set of weighting functions and is required to be orthogonal to the weighting functions in the global sense; that is,

$$
\int_{\Omega} \mathbf{W} \cdot \mathbf{R}(\widetilde{\phi}) d \Omega=0,
$$

where $\mathbf{W}=\left(W_{1}, W_{2}, \ldots W_{n}\right)$ is a set of weighting functions.

An approximate solution may be then be sought in the finite-dimensional form

$$
\phi(x, y, t) \approx \widetilde{\phi}(x, y, t)=\sum_{j=1}^{n} a_{j}(t) N_{j}(x, y)
$$

in which $N_{j}(x, y) \quad(j=1, \ldots, n)$ is a set of basis functions and it is required to find the coefficients $\mathbf{a}_{j}(t)$.

Setting $\mathbf{W}$ equal to each of the weighting functions in turn, a set of algebraic equations can be generated, and these can be solved for the unknown coefficients, and hence an approximation to the solution.

Different versions of the method result from the choice of different classes of weighting functions. In the Galerkin method, the weighting functions are set equal to the basis functions $N_{j}(x, y)$. Thus equation (5.4) becomes

$$
\int_{\Omega} N_{i}(\mathbf{A}(\tilde{\phi})-\mathbf{f}) d \Omega=0, \text { for } i=1 \text { to } n
$$

or

$$
\int_{\Omega} N_{i}\left[\mathbf{A}\left(\sum_{j=1}^{n} \mathbf{a}_{j} N_{j}\right)-\mathbf{f}\right] d \Omega=0, \text { for } i=1 \text { to } n
$$

\subsection{Spatial discretization and weak formulation}

If $\mathbf{A}$ is taken to be the operator defined by equations (4.18), (4.33) and (4.34) in Chapter 4, then (5.6) becomes, for $i=1, \ldots, n$,

for the continuity equation,

$$
\int_{\Omega} W_{i}\left[\frac{\partial r^{m} u}{\partial r}+\frac{\partial r^{m} v}{\partial y}\right] d \Omega=0
$$


for conservation of momentum in the horizontal $(x)$ or radial direction $(r)$,

$$
\int_{\Omega} W_{i}\left[\begin{array}{c}
\frac{\partial u}{\partial t}+u \frac{\partial u}{\partial r}+v \frac{\partial u}{\partial y}+\frac{1}{\rho} \frac{\partial p}{\partial r}+2 \frac{\nu_{e f f}}{r^{2}} u m \\
-\frac{1}{r^{m}} \frac{\partial}{\partial r}\left(r^{m} \nu_{e f f} \frac{\partial u}{\partial r}\right) d \Omega-\frac{1}{r^{m}} \frac{\partial}{\partial y}\left(r^{m} \nu_{e f f} \frac{\partial u}{\partial y}\right) \\
-\frac{1}{r^{m}} \frac{\partial}{\partial r}\left(r^{m} \nu_{e f f} \frac{\partial u}{\partial r}\right)-\frac{1}{r^{m}} \frac{\partial}{\partial y}\left(r^{m} \nu_{e f f} \frac{\partial v}{\partial r}\right)
\end{array}\right] d \Omega=0 ;
$$

and for conservation of momentum in the vertical direction $(\mathrm{y})$

$$
\int_{\Omega} W_{i}\left[\begin{array}{c}
\frac{\partial v}{\partial t}+u \frac{\partial v}{\partial r}+v \frac{\partial v}{\partial y}-\frac{1}{\rho} \frac{\partial p}{\partial y}-g \frac{\rho-\rho_{r}}{\rho} \\
-\frac{1}{r^{m}} \frac{\partial}{\partial r}\left(r^{m} \nu_{e f f} \frac{\partial v}{\partial r}\right)-\frac{1}{r^{m}} \frac{\partial}{\partial y}\left(r^{m} \nu_{e f f} \frac{\partial v}{\partial y}\right) \\
-\frac{1}{r^{m}} \frac{\partial}{\partial r}\left(r^{m} \nu_{e f f} \frac{\partial u}{\partial y}\right)-\frac{1}{r^{m}} \frac{\partial}{\partial y}\left(r^{m} \nu_{e f f} \frac{\partial v}{\partial y}\right)
\end{array}\right] d \Omega=0 .
$$

The scalar equations for the variables $c, k$ and $\varepsilon$ (equations (4.41), (4.74) and (4.75)) are all similar in their formulation and we therefore show only the details for the concentration equation, for which

$$
\int_{\Omega} W_{i}\left[\begin{array}{c}
\frac{\partial c}{\partial t}+u \frac{\partial c}{\partial r}+v \frac{\partial c}{\partial y}-\frac{1}{r^{m}} \frac{\partial}{\partial y}\left(r^{m} V_{S} c\right) \\
-\frac{1}{r^{m}} \frac{\partial}{\partial r}\left(r^{m} \frac{\nu_{e f f}}{\sigma_{S}} \frac{\partial c}{\partial r}\right)-\frac{1}{r^{m}} \frac{\partial}{\partial y}\left(r^{m} \frac{\nu_{e f f}}{\sigma_{S}} \frac{\partial c}{\partial y}\right)
\end{array}\right] d \Omega=0 .
$$

The next key step is that of integration by parts and use of the divergence theorem, which leads to the following expressions:

momentum in the horizontal $(x)$ or radial direction (r)

$$
\int_{\Omega}\left[\begin{array}{c}
W_{i}\left(\frac{\partial u}{\partial t}+u \frac{\partial u}{\partial r}+v \frac{\partial u}{\partial y}+\frac{1}{\rho} \frac{\partial p}{\partial r}+2 \frac{\nu_{e f f}}{r^{2}} u m\right) \\
+\frac{1}{r^{m}} \frac{\partial W_{i}}{\partial r} r^{m} \nu_{e f f} \frac{\partial u}{\partial r}+\frac{1}{r^{m}} \frac{\partial W_{i}}{\partial y} r^{m} \nu_{e f f} \frac{\partial u}{\partial y} \\
+\frac{1}{r^{m}} \frac{\partial W_{i}}{\partial r} r^{m} \nu_{e f f} \frac{\partial u}{\partial r}+\frac{1}{r^{m}} \frac{\partial W_{i}}{\partial y} r^{m} \nu_{e f f} \frac{\partial v}{\partial r}
\end{array}\right] d \Omega=S_{u}
$$


momentum in the vertical direction (y)

$$
\int_{\Omega}\left[\begin{array}{c}
W_{i}\left(\frac{\partial v}{\partial t}+u \frac{\partial v}{\partial r}+v \frac{\partial v}{\partial y}+\frac{1}{\rho} \frac{\partial p}{\partial y}+g \frac{\rho-\rho_{r}}{\rho}\right) \\
+\frac{1}{r^{m}} \frac{\partial W_{i}}{\partial r} r^{m} \nu_{e f f} \frac{\partial v}{\partial r}+\frac{1}{r^{m}} \frac{\partial W_{i}}{\partial y} r^{m} \nu_{e f f} \frac{\partial v}{\partial y} \\
+\frac{1}{r^{m}} \frac{\partial W_{i}}{\partial r} r^{m} \nu_{e f f} \frac{\partial u}{\partial y}+\frac{1}{r^{m}} \frac{\partial W_{i}}{\partial y} r^{m} \nu_{e f f} \frac{\partial v}{\partial y}
\end{array}\right] d \Omega=S_{v}
$$

Here $S_{u}$ and $S_{v}$ are the integrals over the surface $\Gamma$, and are given by

$$
S_{u}=\oint_{\Gamma} W_{i}\left[\nu_{e f f}\left(\frac{\partial u}{\partial r}+\frac{\partial v}{\partial y}\right) n_{r}+\nu_{e f f}\left(\frac{\partial u}{\partial y}+\frac{\partial v}{\partial r}\right) n_{y}\right] d \Gamma
$$

and

$$
S_{V}=\oint_{\Gamma} W_{i}\left[\nu_{e f f}\left(\frac{\partial u}{\partial y}+\frac{\partial v}{\partial r}\right) n_{r}+\nu_{e f f}\left(\frac{\partial u}{\partial r}+\frac{\partial v}{\partial y}\right) n_{y}\right] d \Gamma,
$$

where $n_{x}$ and $n_{y}$ are the components of the outward unit normal vector to $\Gamma$, as shown in Figure 5.2.

For the scalar equation of the concentration $c$ (and likewise for the other scalar variables $k$ and $\varepsilon$ ) we have

$$
\int_{\Omega}\left[\begin{array}{c}
W_{i}\left(\frac{\partial c}{\partial t}+u \frac{\partial c}{\partial r}+v \frac{\partial c}{\partial y}-\frac{1}{r^{m}} \frac{\partial}{\partial y}\left(r^{m} V_{S} c\right)\right) \\
+\frac{1}{r^{m}} \frac{\partial W_{i}}{\partial r} r^{m} \frac{\nu_{e f f}}{\sigma_{S}} \frac{\partial c}{\partial r}+\frac{1}{r^{m}} \frac{\partial W_{i}}{\partial y} r^{m} \frac{\nu_{e f f}}{\sigma_{S}} \frac{\partial c}{\partial y}
\end{array}\right] d \Omega=S_{c},
$$

where $S_{c}$ is the surface integral given by

$$
S_{c}=\oint_{\Gamma} W_{i}\left[\frac{\nu_{e f f}}{\sigma_{S}}\left(\frac{\partial c}{\partial r} n_{r}+\frac{\partial c}{\partial y} n_{y}\right)\right] d \Gamma .
$$

Equations (5.12) - (5.17) are referred to as a weak formulation of the problem, since the differentiality requirements on the unknown variables have been reduced or weakened.

We now choose basis function $\left(N_{1}, N_{2}, \ldots N_{n}\right)$ for the velocities $u$ and $v$, and write

$$
u=\sum_{j=1}^{n}[u(t)]_{j} N_{j}, v=\sum_{j=1}^{n}[v(t)]_{j} N_{j}
$$


Likewise, we write

$$
p=\sum_{j=1}^{l}[p(t)]_{j} L_{j}, c=\sum_{j=1}^{m}[c(t)]_{j} M_{j}
$$

where $L_{j}$ and $M_{j}$ are the basis functions associated with the pressure $p$ and concentration $c$, respectively. Similar considerations apply to the turbulent variables $k$ and $\varepsilon$.

If the general form of these solutions is used in the weak formulation with the weighting function $W$ chosen to be the basis functions $N_{i}$ or $M_{i}$, then these equations become

$$
\begin{aligned}
& \int_{\Omega} \sum_{i=1}^{n} \sum_{j=1}^{n} N_{i}\left[\frac{\partial r^{m} N_{j}}{\partial r} u_{j}+\frac{\partial r^{m} N_{j}}{\partial y} v_{j}\right] d \Omega=0, \\
& {\left[N_{i}\left(\frac{\partial N_{j}}{\partial t} u_{j}+\sum_{k=1}^{n} N_{k} u_{k} \frac{\partial N_{j}}{\partial r} u_{j}+\sum_{k=1}^{n} N_{k} v_{k} \frac{\partial N_{j}}{\partial y} v_{j}\right)\right.} \\
& +N_{i}\left(2 \frac{\nu_{e f f}}{r^{2}} N_{j} u_{j} m+\frac{1}{\rho} \frac{\partial L_{k}}{\partial r} p_{k}\right)
\end{aligned}
$$

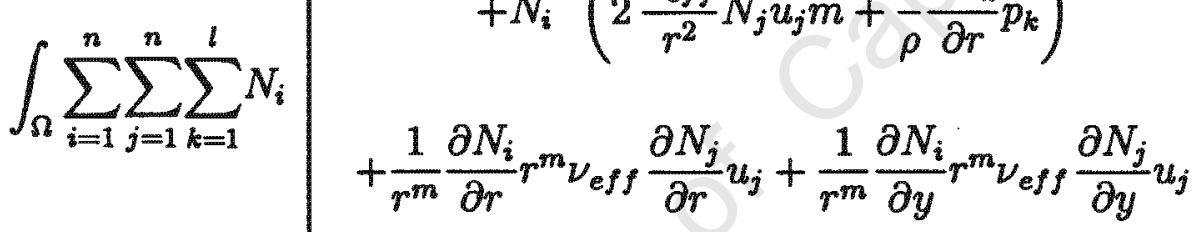

$$
\begin{aligned}
& +\frac{1}{r^{m}} \frac{\partial N_{i}}{\partial r^{m}} r^{m} \nu_{e f f} \frac{\partial N_{j}}{\partial r} u_{j}+\frac{1}{r^{m}} \frac{\partial N_{i}}{\partial y} r^{m} \nu_{e f f} \frac{\partial N_{j}}{\partial r} v_{j} \\
& {\left[N_{i}\left(\frac{\partial N_{j}}{\partial t} v_{j}+\sum_{k=1}^{n} N_{k} u_{k} \frac{\partial N_{j}}{\partial r} v_{j}+\sum_{k=1}^{n} N_{k} v_{k} \frac{\partial N_{j}}{\partial y} V_{j}\right)\right.} \\
& +N_{i}\left(\frac{1}{\rho} \frac{\partial L_{k}}{\partial y} p_{k}+N_{j}\left(g \frac{\rho-\rho_{r}}{\rho}\right)\right) \\
& \int_{\Omega} \sum_{i=1}^{n} \sum_{j=1}^{n} \sum_{k=1}^{l} N_{i} \\
& +\frac{1}{r^{m}} \frac{\partial N_{i}}{\partial r} r^{m} \nu_{e f f} \frac{\partial N_{j}}{\partial r} v_{j}+\frac{1}{r^{m}} \frac{\partial N_{i}}{\partial y} r^{m} \nu_{e f f} \frac{\partial N_{j}}{\partial y} v_{j} \\
& \left.+\frac{1}{r^{m}} \frac{\partial N_{i}}{\partial r} r^{m} \nu_{e f f} \frac{\partial N_{j}}{\partial y} u_{j}+\frac{1}{r^{m}} \frac{\partial N_{i}}{\partial y} r^{m} \nu_{e f f} \frac{\partial N_{j}}{\partial y} v_{j}\right] \\
& d \Omega=S_{v}
\end{aligned}
$$

in which the surface integrals $S_{u}$ and $S_{v}$ are now

$$
S_{u}=\oint_{\Gamma} \sum_{i=1}^{n} \sum_{j=1}^{n} N_{i}\left[\nu_{e f f}\left(\frac{\partial N_{j}}{\partial x} u_{j}+\frac{\partial N_{j}}{\partial y} v_{j}\right) n_{x}+\nu_{e f f}\left(\frac{\partial N_{j}}{\partial y} u_{j}+\frac{\partial N_{j}}{\partial x} v_{j}\right) n_{y}\right] d \Gamma
$$


and

$S_{v}=\oint_{\Gamma} \sum_{i=1}^{n} \sum^{n} N_{i}\left[\nu_{e f f}\left(\frac{\partial N_{j}}{\partial y} u_{j}+\frac{\partial N_{j}}{\partial x} v_{j}\right) n_{x}+\nu_{e f f}\left(\frac{\partial N_{j}}{\partial x} u_{j}+\frac{\partial N_{j}}{\partial y} v_{j}\right) n_{y}\right] d \Gamma$.

For convenience we have written $[u(t)]_{j} \equiv u_{j}$, etc.

For the equation for the concentration $c$ (likewise for the other scalar variables $k$ and $\varepsilon$ ) we have

$$
\int_{\Omega} \sum_{i=1}^{n} \sum_{j=1}^{m} M_{i}\left[\begin{array}{c}
M_{i}\left(\frac{\partial M_{j}}{\partial t} c_{j}+\sum_{k=1}^{n} N_{k} u_{k} \frac{\partial M_{j}}{\partial r} c_{j}\right) \\
+M_{i}\left(\sum_{k=1}^{n} N_{k} v_{k} \frac{\partial M_{j}}{\partial y} c_{j}-\frac{1}{r^{m}} \frac{\partial M_{j}}{\partial y}\left(r^{m} V_{S} c_{j}\right)\right) \\
+\frac{1}{r^{m}} \frac{\partial M_{i}}{\partial r} r^{m} \frac{\nu_{e f f}}{\sigma_{S}} \frac{\partial M_{j}}{\partial r} c_{j}+\frac{1}{r^{m}} \frac{\partial M_{i}}{\partial y} r^{m} \frac{\nu_{e f f}}{\sigma_{S}} \frac{\partial M_{j}}{\partial y} c_{j}
\end{array}\right] d \Omega=S_{c}
$$

where $S_{c}$ is now given by

$$
S_{c}=\oint_{\Gamma} \sum_{i=1}^{m} \sum_{j=1}^{m} M_{i}\left[\frac{\nu_{e f f}}{\sigma_{S}}\left(\frac{\partial M_{j}}{\partial x} c_{j} n_{x}+\frac{\partial M_{j}}{\partial y} c_{j} n_{y}\right)\right] d \Gamma
$$

The continuity and momentum equations (5.20) - (5.24) and the concentration equation (5.25) with (5.26) can be expressed in the matrix form

$$
\begin{gathered}
\mathbf{G} \underline{u}=\mathbf{0} \\
\mathbf{M}_{\mathbf{u}} \frac{\partial \underline{u}}{\partial t}+[\mathbf{C}+\mathbf{K}] \underline{u}=-\mathbf{G}^{\mathbf{T}} \underline{p}+\mathbf{F}_{\underline{u}}, \\
\mathbf{M}_{\mathbf{c}} \frac{\partial \underline{c}}{\partial t}+\left[\mathbf{C}_{\mathbf{c}}+\mathbf{K}_{\mathbf{c}}\right] \underline{c}=\mathbf{F}_{c}
\end{gathered}
$$

with

$$
\begin{gathered}
\underline{u}=\left[u_{1}, \ldots u_{n}\right] ; \underline{c}=\left[c_{1}, \ldots c_{m}\right] \text { and } \underline{p}=\left[p_{1}, \ldots p_{l}\right] \\
\mathbf{G}=\int_{\Omega}(\nabla \mathbf{N})^{\mathbf{T}} \mathbf{N} d \Omega \\
\mathbf{M}_{\mathbf{u}}=\int_{\Omega} \mathbf{N}^{\mathbf{T}} \mathbf{N} d \Omega \text { and } \mathbf{M}_{\mathbf{c}}=\int_{\Omega} \mathbf{M}^{\mathbf{T}} \mathbf{M} d \Omega \\
\mathbf{K}=\int_{\Omega} \nu_{e f f}(\nabla \mathbf{N})^{\mathbf{T}}(\nabla \mathbf{N}) d \Omega \text { and } \mathbf{K}_{\mathbf{c}}=\int_{\Omega} \frac{\nu_{\text {eff }}}{\sigma_{S}}(\nabla \mathbf{M})^{\mathbf{T}}(\nabla \mathbf{M}) d \Omega \\
\mathbf{C}=\int_{\Omega} \mathbf{N}^{\mathbf{T}}(\nabla(\underline{u} \mathbf{N})) d \Omega \text { and } \mathbf{C}_{\mathbf{c}}=\int_{\Omega} \mathbf{M}^{\mathbf{T}}(\nabla(\underline{u} \mathbf{M})) d \Omega
\end{gathered}
$$




$$
\begin{gathered}
\mathbf{F}_{\underline{u}}=\int_{\Omega} \mathbf{N}^{\mathbf{T}}\left(g_{y} \frac{\rho-\rho_{r}}{\rho}\right) d \Omega+\int_{\Gamma} \mathbf{N}^{\mathbf{T}} \boldsymbol{T} d \Gamma \\
\mathbf{F}_{c}=\int_{\Gamma} \mathbf{N}^{\mathbf{T}} \mathbf{q} d \Gamma .
\end{gathered}
$$

Here $\tau$ is the outward normal component of the velocity flux, and $q$ is the outward normal component of the suspended solid flux.

The matrix form of the scalar equations for $k$ and $\varepsilon$ can be written in an analogous way.

\subsection{The finite element method}

The question of how to define the shape functions is where the technique of finite element methodology really begins. The finite element method subdivides the domain into a set of discrete volumes or finite elements, on which the solution is approximated by the basis functions. In principle the first step for all these numerical approaches is to define a grid consisting of a finite number of nonoverlapping subintervals that cover the whole domain, where each subintervall is called an "element". In two dimensions the elements are typically triangles or quadrilaterals and provision can be made for curvilinear edges. Nodal points are defined independently and distributed throughout the domain $\Omega$ and on its boundary, though nodes are required at least at element vertices. Figure 5.2 gives some examples of elements and nodes.

The basis functions $N_{i}$ (likewise also $M_{i}$ and $L_{i}$ ) for the finite element method are defined as follows:

(a) $N_{i}$ is continuous;

(b) the basis function $N_{i}$ is equal to 1 at node $i$, and equal to zero at the other nodes; that is,

$$
N_{i}\left(\underline{x}_{j}\right)=\delta_{i j}=\left\{\begin{array}{c}
1 \text { if } i=j \\
0 \text { otherwise }
\end{array},\right.
$$

where $\underline{x}_{i}$ is the set of coordinates of node $i$; 

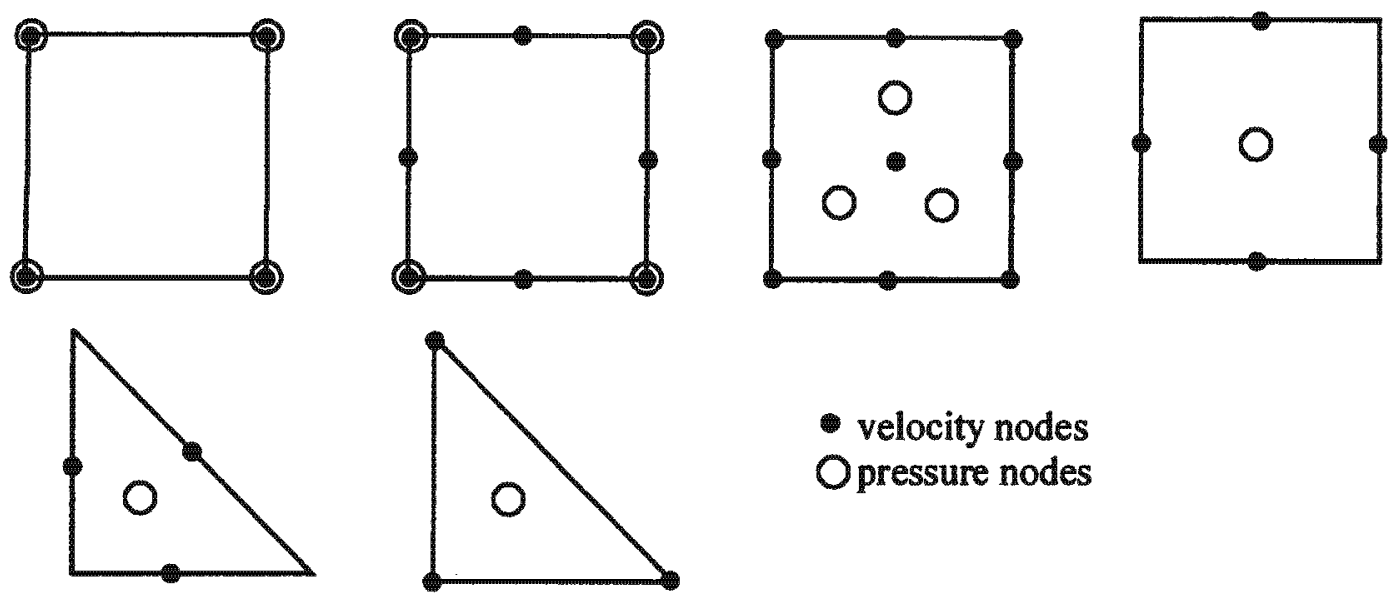

velocity nodes

Opressure nodes

Figure 5.2: Typical velocity-pressure elements for continuous and discontinuous pressure.

(c) in each element $\Omega_{e}$ the local basis function $N_{i}^{(e)}$, defined to be the restriction of $N_{i}$ to element $e$, is a polynomial of some degree $k$; that is,

$$
N_{i}^{(e)} \equiv N_{i}^{(e)} \mid \Omega_{e} \in P_{k}\left(\Omega_{e}\right) \text { for some } k \geq 1
$$

where $P_{k}\left(\Omega_{e}\right)$ is the set of polynomials of degree at most $k$ on $\Omega_{e}$.

The approximate solution of the variable $\underline{u}$ on $\Omega_{e}$, for example, can then be written as

$$
\left.\underline{u}\right|_{\Omega_{s}}=\sum_{i=1}^{n} \underline{u}_{i}^{(e)} N_{i}^{(e)}
$$

where $\underline{u}_{i}^{(e)}$ is the value of $\underline{u}$ at node $i$.

\section{Mapping from a reference element}

Instead of defining local basis functions for each element, it is more convenient to set up a reference element $\hat{\Omega}$; basis functions are then defined on the reference element and then mapped to the actual element.

For convenience we develop the necessary theory in the context of two dimensions, and for the four-noded quadrilateral element, which will be used later in all computations. In this case the reference element is the square $\{(\xi, \eta) \mid-1 \leq$ $\xi, \eta \leq 1\}$ (Figure 5.3). 


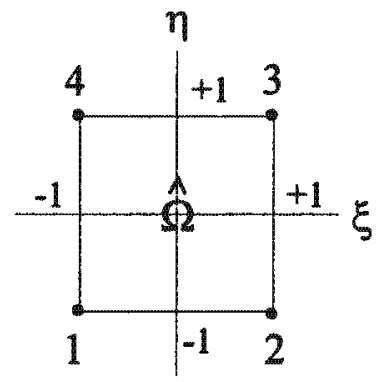

Figure 5.3: The reference element for the four-node $C^{0}$-linear isoparametric quadrilateral.

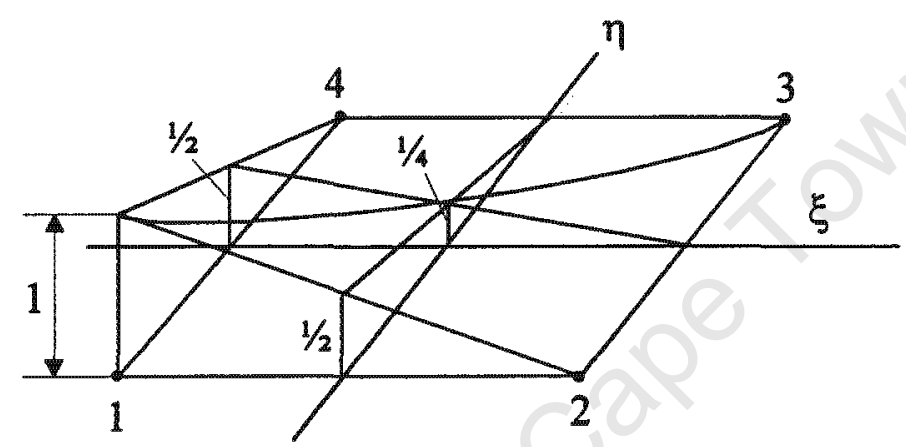

Figure 5.4: The reference basis function $\hat{N}_{1}(\xi, \eta)$ for the four-node $C^{0}$-linear isoparametric quadrilateral.

Next, we define on $\hat{\Omega}$ the local basis functions $\hat{N}_{i}$ by

$$
\hat{N}_{i}=\frac{1}{4}\left(1+\xi_{i} \xi\right)\left(1+\eta_{i} \eta\right) \quad \text { for } i=1, \ldots, 4,
$$

where $\left(\xi_{i}, \eta_{i}\right)$ are the coordinates of node $i$ (see Figure 5.4). The polynomials in this case are bilinear, that is, linear separately in $\xi$ and $\eta$ (or in $x$ and $y$ ).

Next, the map from the reference element to an actual element $\Omega_{e}$ may now be easily defined by

$$
x=\sum_{i=1}^{n} x_{i} \hat{N}_{i} \text { and } y=\sum_{i=1}^{n} y_{i} \hat{N}_{i}
$$

This is known as an isoparametric map, as the same basis functions are used to define the unknown function and the element maps. Finally, we also have, on $\Omega_{e}$,

$$
N_{i}^{(e)}(x, y)=\hat{N}_{i}(\xi, \eta)
$$


where $(x, y)$ and $(\xi, \eta)$ are related to each other through (5.40).

Given the map (5.40) between the reference and actual elements, it is possible, using the chain rule, to evaluate basis functions and their derivatives on the reference element. First we define the Jacobian matrix $\mathbf{J}$ by

$$
\mathbf{J}=\left[\begin{array}{ll}
\frac{\partial x}{\partial \xi} & \frac{\partial y}{\partial \xi} \\
\frac{\partial x}{\partial \eta} & \frac{\partial y}{\partial \eta}
\end{array}\right]
$$

Then we have

$$
\left[\begin{array}{c}
\frac{\partial \hat{N}_{i}}{\partial \xi} \\
\frac{\partial \hat{N}_{i}}{\partial \eta}
\end{array}\right]=\left[\begin{array}{ll}
\frac{\partial x}{\partial \xi} & \frac{\partial y}{\partial \xi} \\
\frac{\partial x}{\partial \eta} & \frac{\partial y}{\partial \eta}
\end{array}\right]\left[\begin{array}{c}
\frac{\partial N_{i}^{(e)}}{\partial x} \\
\frac{\partial N_{i}^{(e)}}{\partial y}
\end{array}\right]=\mathbf{J}\left[\begin{array}{c}
\frac{\partial N_{i}^{(e)}}{\partial x} \\
\frac{\partial N_{i}^{(e)}}{\partial y}
\end{array}\right] .
$$

To find the derivatives of $N_{i}$ equation (5.43) may be inverted to give

$$
\left[\begin{array}{c}
\frac{\partial N_{i}^{(e)}}{\partial x} \\
\frac{\partial N_{i}^{(e)}}{\partial y}
\end{array}\right]=\mathbf{J}^{-1}\left[\begin{array}{c}
\frac{\partial \hat{N}_{i}}{\partial \xi} \\
\frac{\partial \hat{N}_{i}}{\partial \eta}
\end{array}\right]=\frac{1}{\operatorname{det} \mathbf{J}}\left[\begin{array}{cc}
\frac{\partial y}{\partial \eta} & -\frac{\partial y}{\partial \xi} \\
-\frac{\partial x}{\partial \eta} & \frac{\partial x}{\partial \xi}
\end{array}\right]\left[\begin{array}{c}
\frac{\partial \hat{N}_{i}}{\partial \xi} \\
\frac{\partial \hat{N}_{i}}{\partial \xi}
\end{array}\right],
$$

where $\operatorname{det} \mathbf{J}$ denotes the determinant of $\mathbf{J}$ and is given by

$$
\operatorname{det} \mathbf{J}=\frac{\partial x}{\partial \xi} \frac{\partial y}{\partial \eta}-\frac{\partial x}{\partial \eta} \frac{\partial y}{\partial \xi} .
$$

Thus we have

$$
\left[\begin{array}{cc}
\frac{\partial \xi}{\partial x} & \frac{\partial \xi}{\partial x} \\
-\frac{\partial \eta}{\partial x} & \frac{\partial \eta}{\partial y}
\end{array}\right]=\frac{1}{\operatorname{det} \mathbf{J}}\left[\begin{array}{cc}
\frac{\partial y}{\partial \eta} & -\frac{\partial y}{\partial \xi} \\
-\frac{\partial x}{\partial \eta} & \frac{\partial x}{\partial \xi}
\end{array}\right]
$$

The differential area transforms according to the relation

$$
d \Omega=d x d y=\operatorname{det} \mathbf{J} d \xi d \eta .
$$




\section{Numerical integration}

The formulation of integrals in terms of $\xi$ and $\eta$ yields simple integration limits. The integrals are defined as

$$
\iint_{\Omega} F(x, y) d x d y=\int_{-1}^{1} \int_{-1}^{1} f(\xi, \eta) \operatorname{det} \mathbf{J} d \xi d \eta,
$$

and it is convenient in the computational procedure to carry out all integrations approximately, using numerical quadrature.

Generally, the basis of most numerical integration schemes is the identification of selected sampling points at which the value of the function is sampled, and the specification of a set of weights, one for each sampling point. In order to achieve advanced accuracy the sampling points should be located at points to be determined where best accuracy can be attained. The choice is carried out in such a way that the integration scheme is exact for polynomials of a given degree. Gauss-Legendre quadrature is a popular and accurate option for integration of functions over the interval $(-1,1)$. The integration over the reference element using Gauss-Legendre quadrature of order $G n$ is defined as

$$
\int_{-1}^{1} \int_{-1}^{1} f(\xi, \eta) \operatorname{det} J d \xi d \eta \cong \sum_{i=1}^{G} \sum_{j=1}^{n} w_{i} w_{j} f\left(\xi_{i}, \eta_{j}\right) .
$$

Here $n$ is the number of integration points, $w_{i}$ and $w_{j}$ are the weighting factors, and the sampling points are denoted by $\xi_{i}$ and $\eta_{j}$. With correct choice of the sampling points and of the weights, the Gauss method integrates the product of a $(2 G-1)$ polynomial in $\xi$-direction by a $(2 n-1)$ polynomial in $\eta$-direction, exactly. In order to ensure exact evaluation of the entries in the matrices for the rectangular element the choice of $G=n=2$ must be made, that is the $2 \times 2$ integration rule. The weighting factors $w_{i}$ are equal to 1 and the sampling points are at $\pm 1 / \sqrt{3}$ in both directions.

\subsubsection{Restrictions on the choice of velocity-pressure ele- ments}

Focussing for the moment on the Navier-Stokes problem, that is, equations (4.33) and (4.34), the solvability of this set of equations depends crucially on the choice 


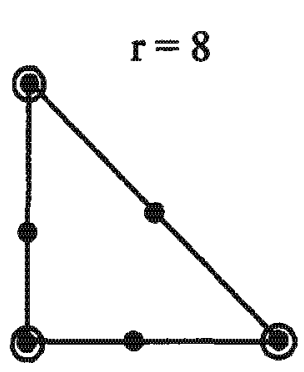

(a)

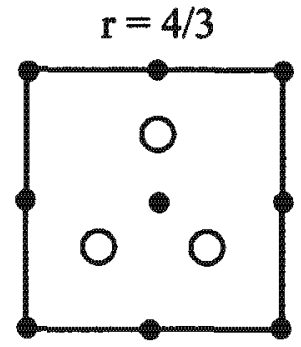

(b)

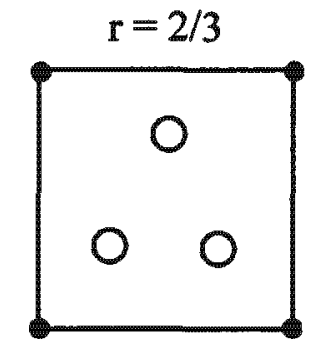

severe mesh locking

(c)

- velocity nodes

Opressure nodes

Figure 5.5: The quadratic-linear (a) and biquadratic-linear (b) elements satisfy the BB condition, while the bilinear velocity-linear pressure element (c) does not.

of elements for the velocity and pressure. The pair of equations constitutes a mixed problem; the fundamental theory on necessary and sufficient conditions for unique solvability of the equations for mixed or saddle-point problems were first proposed by Babuska (1971) and Brezzi (1974). Their theory results in restrictions on the choice of combinations of the finite element interpolations used to approximate the velocity and pressure fields. The key condition that has to be satisfied is the so-called Babuska-Brezzi stability condition. The use of combinations of elements that do not satisfy the Babuska-Brezzi condition may lead to undesirable features such as volumetric locking, in which the velocity goes to zero as the mesh is refined, and the existence of spurious pressure modes.

Figures 5.5 (a) and (b) show triangular and quadrilateral elements for which the $\mathrm{BB}$ condition is satisfied; these correspond respectively to velocity-pressure approximations that are (a) quadratic-linear and (b) biquadratic-linear. In the latter case the pressures are discontinuous across element boundaries. Figure 5.5 (c) shows an element that does not satisfy the BB condition: this uses a bilinear velocity-linear pressure combination. Further examples of stable and unstable element choices may be found in Hughes (1987).

Various techniques have been developed to test for satisfaction of the BB condition, and to develop elements that satisfy the condition. Examples of such works are those of Sani et al. (1981), Rice \& Schnipke (1984), Franca et al. (1992), and Franca \& Frey (1992). 
The issue of solvability may be better understood by considering, for simplicity, the steady linear Stokes problem, in which equations (4.18) to (4.36) and reduce to

$$
\left[\begin{array}{cc}
\mathbf{K} & \mathbf{G}^{T} \\
\mathbf{G} & 0
\end{array}\right]\left\{\begin{array}{l}
\underline{u} \\
\underline{p}
\end{array}\right\}=\left\{\begin{array}{l}
\mathbf{f} \\
0
\end{array}\right\},
$$

where $\underline{u}$ is the primary variable and $\underline{p}$ is the constraint variable. Eliminating $\underline{u}$ from the first equation and substituting into the second of equation 5.50 , we obtain

$$
\left(\mathbf{G K}^{-1} \mathbf{G}^{T}\right) \underline{p}=\mathbf{G K}^{-1} \mathbf{f} .
$$

It is obvious that the matrix $\mathbf{K}$ has to be non-singular, and in order to solve for $\underline{p}$ the expression in brackets must fulfil the same restriction. The matrix $\left(\mathbf{G K}^{-1} \mathbf{G}^{T}\right)$ will always be singular when its rank, which needs to be the number $n_{p}$ of the unknowns of $\underline{p}$, is less than the rank of $\mathbf{K}^{-1}$, which is the number $n_{u}$ of the unknowns of $\underline{u}$, that is,

$$
n_{u} \geq n_{p}
$$

It is a necessary though not sufficient requirement. Defining the constraint ratio $r=n_{u} / n_{p}$ the following statements for two dimensions can be made (see Hughes 1987):

$$
\begin{aligned}
& r>2 \text { too few incompressibility constraints } \\
& r=2 \text { optimal } \\
& r<2 \text { too many incompressibility constraints } \\
& r \leq 1 \text { locking. }
\end{aligned}
$$

Some examples of mixed elements with different constraint ratios are shown in Figure 5.5.

It is, however, possible to circumvent the BB condition by exploiting terms that arise in the time-discretization of the problem; this is discussed in further detail in Section 5.6.

\subsubsection{Difficulties with the classical Galerkin method}

Some important remarks should be made about the problems that can arise when using the classical Galerkin finite element method. In the context of the Navier-Stokes equations spurious oscillations in the solution may arise from two sources: 
(a) High Reynolds and Peclet numbers

This gives rise to situations in which convective effects dominate over diffusive effects. One possible way of eliminating the oscillations is to severely refine the mesh, so that convection no longer dominates at element level. A further possibility is the use of upwinding techniques, such as the Streamline-Upwind Petrov-Galerkin method (SUPG). This method, introduced by Brooks \& Hughes (1982), is a modification of the classical Galerkin method in which the weighting function is modified so as to weigh the element upwind of the node more heavily. The basis functions and the weighting functions are thus distinct, and the weighting functions, which are the sum of the continuous weighting functions used in the classical Galerkin method and a discontinuous streamline upwind contribution, are discontinuous. The magnitude of this perturbation depends on the directional Peclet number of an element, which in turn depends on the local flow velocity in the centre and the largest length dimension of the element.

\section{(b) The presence of gravity}

The gravity term leads to a problem that is no longer purely convective-diffusive in nature. In stratified flow fields, such as in settling tanks, the gravity term may reduce the efficiency of the upwinding scheme. In fact this can lead to serious convergence problems, and exerts a strong influence on the flow field in a settling tank.

Extensive testing has shown that the use of the equal-order element pair described in Section 5.6 in the range of problems considered in this work leads to stable results, so that no upwinding was necessary.

\subsection{Time discretization}

Consider the system of linear ordinary differential equations (ODEs) in time given by

$$
A \frac{d \phi}{d t}+B \phi+C=0,
$$

where $\phi$ is in general a vector function and $A, B$ and $C$ are in general functions of time. This system is supplemented by an initial condition

$$
\phi(0)=\phi_{0}
$$


It is required to solve (5.53) and (5.54) on a time interval $(0, T)$, for some time $T$. In order to find an approximate solution to this initial-value problem the time interval $[0, T]$ is partitioned into subintervals according to

$$
0=t_{0}<t_{1}<t_{2} \ldots<t_{N}=T
$$

It is then required to find approximations of $\phi_{n}$ the solution $\phi\left(t_{n}\right)$, for $n=$ $1, \ldots N$. To do this it is necessary to approximate the derivative in (5.53); typically this is done by replacing the derivative with a finite difference; for example,

$$
\frac{d \phi}{d t} \simeq \frac{\left(\phi^{n+1}-\phi^{n}\right)}{\left(t_{n+1}-t_{n}\right)}
$$

In addition, the term $B(t) \phi$ may be replaced by its value at $t_{n+1}:=t_{n}+$ $\theta\left(t_{n+1}-t_{n}\right)$, for $\theta$ in the range $0 \leq \theta \leq 1$. Assuming for simplicity a uniform partition, so that $\Delta t=t_{n+1}-t_{n}$ for all $n=1, \ldots N$, equation (5.53) may be approximated by $t_{n}$ for all $n=1, \ldots N-1$, equation may be approximated by

$$
A_{n+1} \frac{\phi^{n+1}-\phi^{n}}{t_{n+1}-t_{n}}+B^{n+\theta} \phi^{n+\theta}=0, \quad n=1, \ldots N-1 .
$$

Time discretization schemes can be single- or multi-step: in the former case the values of variables at time $t_{n+1}$ are related to values at time $t_{n}$ within a single time step, while in the latter the values at $t_{n+1}$ are linked to those at $t_{n}, t_{n-1}, t_{n-2}$, etc. (Zienkiewicz et al. 1984, Wood 1984, Hughes et al. 1989, Turek 1998).

In the next section the most common single-step schemes will be described, in the context of the Navier-Stokes equations.

\subsubsection{Single-step schemes}

The spatially discretized Navier-Stokes equations are given by

$$
\begin{aligned}
\mathbf{M}_{\mathbf{m}} \frac{d \underline{u}}{d t}+[\mathbf{C}+\mathbf{K}] \underline{u}+\mathbf{G}^{\mathbf{T}} \underline{p} & =\mathbf{F}_{\underline{u}}, \\
\mathbf{G} \underline{u} & =\mathbf{0},
\end{aligned}
$$

with prescribed boundary values and initial conditions. Discretization in time along the lines described above leads to the system

$$
\begin{aligned}
\mathbf{M}_{\mathbf{m}}\left(\frac{\underline{u}^{n+1}-\underline{u}^{n}}{\Delta t}\right)+\theta\left[\left(\mathbf{C}^{n+1}+\mathbf{K}^{n+1}\right) \underline{u}^{n+1}+\mathbf{G}^{\mathbf{T}} \underline{p}^{n+1}\right] & =\mathbf{A}^{n+1} \\
\mathbf{G} \underline{u}^{n+1} & =0
\end{aligned}
$$


with the right-hand side given by

$$
\mathbf{A}^{n+1}:=\theta \mathbf{F}_{\underline{u}}^{n+1}+(1-\theta) \mathbf{F}_{\underline{u}}^{n}-(1-\theta)\left[\left(\mathbf{C}^{n}+\mathbf{K}^{n}\right) \underline{u}^{n}+\mathbf{G}^{\mathbf{T}} \underline{p}^{n}\right] .
$$

Here the nonlinear nature of the matrix $\mathbf{C}$ is suppressed, since eventually the solution algorithm will incorporate a linearization scheme.

The most commonly used single-step schemes are:

- $\theta=1$ the explicit forward Euler scheme

- $\theta=0 \quad$ the implicit backward Euler scheme

- $\theta=\frac{1}{2} \quad$ the Crank-Nicolson scheme

The parameter $\theta$ may used to control the stability and accuracy of the approximation. It is well known that for $\theta<1 / 2$ the method is only conditionally stable, while the scheme is unconditionally stable for $\theta \geq 1 / 2$ (see the next subsection).

Many problems in fluid mechanics are of widely varying time scales and the slow, long-term behaviour of the solution is of interest, for example in the special case of flows in secondary settling tanks. Such problems with a wide range of time scales are called stiff, and they are one of the biggest challenges in computational fluid dynamics. It is therefore important to investigate the behaviour of methods for large time steps.

To ensure convergence it is necessary that the approximation fulfils consistency and stability conditions. The consistency requirement ensures that the timediscrete approximation, if it converges, will do so to the exact solution, as the time step tends to zero. The stability condition requires that the solution of the discrete equation system is unique and that spurious mechanisms, which can pollute the solution, are absent.

The explicit Euler forward scheme is the simplest method and easy to program, but because it is conditionally stable it requires very small step sizes and has only a first-order truncation error. The backward Euler scheme allows large time steps to be taken, but this scheme tends to produce smooth solutions even when $\Delta t$ is very large. Other shortcomings are the need to solve a large coupled set of equations at each time step, the requirement of much more storage than the explicit scheme, and its first-order truncation error in time. The Crank-Nicolson 
scheme is second-order accurate, and requires very little additional computational effort over that of the first-order implicit Euler scheme. However, it has a weak damping property (that is, it is not strongly $A$-stable ${ }^{1}$ ) unexpected instabilities and oscillatory solutions are possible. In summary, the backward Euler scheme is robust, but strongly damping, the Crank-Nicolson scheme is more accurate, but tends to yield instabilities. The following subsection gives an insight into the basic analysis of stability for time discretization schemes.

\subsubsection{Stability considerations}

Returning to equations (5.53) and (5.54), the free response is obtained when $C=0$, and the solution in this case, for constant $\mathrm{A}$ and $\mathrm{B}$, is given by

$$
\phi(t)=\alpha e^{-\lambda t} .
$$

Substitution in (5.53) yields

$$
\alpha(-A \lambda+B) e^{-\lambda t}=0 .
$$

Since this must hold for all $t$, we have

$$
A \lambda+B=0 .
$$

This is known as the characteristic equation and $\lambda=B / A$ is the characteristic value, or eigenvalue. Use of the initial condition results in the expression

$$
\phi(t)=\phi_{0} e^{-A / B\left(t-t_{0}\right)}, \quad t>t_{0} .
$$

Application of the discretization scheme (5.56) to the free response problem leads to the equation

$$
\left(\frac{1}{\Delta t} A+\theta B\right) \phi^{n+1}=\left(\frac{1}{\Delta t} A-(1-\theta) B\right) \phi^{n} .
$$

Substituting $\lambda=B / A$ and multiplying both sides by $\Delta t / A$, we obtain

$$
\omega:=\frac{\phi^{n+1}}{\phi^{n}}=\frac{1-(1-\theta) \lambda \Delta t}{1+\theta \lambda \Delta t},
$$

where $\omega$ is the amplification factor. For local stability of time stepping schemes one requires $\omega<1$.

\footnotetext{
${ }^{1} A$ matrix $A$ is ill-conditioned or unstable when small changes in the coefficients or in the constants can produce very great changes in the solutions.
} 
The stability condition for a system of equations is obtained in the same way, in terms of its eigenvalues $\lambda_{i}$, and the condition is now

$$
\begin{gathered}
\left|\omega_{i}\right|<1 \quad i=1,2, \ldots N, \\
\lambda_{i} \Delta t<\frac{2}{1-2 \theta} \quad \text { for } 0 \leq \theta<\frac{1}{2} \quad i=1,2, \ldots N, \\
\lambda_{i} \Delta t>\frac{-2}{2 \theta-1} \quad \text { for } \theta \geq \frac{1}{2} \quad i=1,2, \ldots N .
\end{gathered}
$$

Equation (5.66) is satisfied since $\lambda_{i}>0$ for $i=1,2, \ldots N$. Thus there are no restrictions on the step size, and methods for which $\theta \geq 1 / 2$ are said to be unconditionally stable. Equation (5.65) poses restrictions on the step size, and methods for which $0 \leq \theta<1 / 2$ are therefore said to be conditionally stable.

The detailed derivation and explanation of the stability conditions for a system of equations in general can be found, for example in Burnett (1987).

\subsubsection{A fractional step scheme}

We present here details of a fractional-step (FS) scheme, which combines the advantages of the backward Euler and Crank-Nicolson schemes. The scheme is of second-order accuracy, preserves physical oscillations, and is strongly A-stable. It has been implemented in the algorithm developed in this work.

The FS scheme was first proposed by Glowinski \& Periaux (1987), and is used in the program package 'FEATFLOW' by Turek (1998). The scheme uses three different values for $\theta$ and for the time step at each level; that is, the macro time step $\Delta t$ is subdivided into three sub-steps of variable size $t_{i}$, so that

$$
\Delta t=\sum_{i=1}^{3} t_{i} .
$$

A sequence of generalized stationary problems is obtained for each time sub-step. The time step $\Delta t$ is split into three consecutive substeps of intervals, which are based on the settings (see Turek 1998):

$$
\begin{aligned}
\theta & =1-\frac{\sqrt{2}}{2} \approx 0.293 \\
\theta^{\prime} & =1-2 \theta \approx 0.414 \\
\tilde{\theta} & =\alpha \theta \Delta t=\beta \theta^{\prime} \Delta t \\
\text { with } \alpha & =\frac{1-2 \theta}{1-\theta} \approx 0.585 \text { and } \beta=1-\alpha \approx 0.414 .
\end{aligned}
$$


The implementation of the scheme is shown here, by way of example, for the Navier-Stokes equations. Using the compact notation $\mathbf{N} \underline{u}:=(\mathbf{C}+\mathbf{K}) \underline{u}$, we have

$$
\begin{aligned}
{\left[\mathbf{M}_{\mathbf{m}}+\tilde{\theta} \mathbf{N}\right] \underline{u}^{n+\theta}+\theta \Delta t \mathbf{G}^{\mathbf{T}} \underline{p}^{n+\theta} } & =\left[\mathbf{M}_{\mathbf{m}}-\beta \theta \Delta t \mathbf{N}\right] \underline{u}^{n}+\theta \Delta t \mathbf{F}^{n}, \\
\mathbf{G} \underline{u}^{n+\theta} & =\mathbf{0}, \\
{\left[\mathbf{M}_{\mathbf{m}}+\tilde{\theta} \mathbf{N}\right] \underline{u}^{n+1-\theta}+\theta^{\prime} \Delta t \mathbf{G}^{\mathbf{T}} \underline{\underline{p}}^{n+1-\theta} } & =\left[\mathbf{M}_{\mathbf{m}}-\alpha \theta^{\prime} \Delta t \mathbf{N}\right] \underline{u}^{n+\theta}+\theta^{\prime} \Delta t \mathbf{F}^{n+1-\theta}, \\
\mathbf{G} \underline{u}^{n+1-\theta} & =\mathbf{0}, \\
{\left[\mathbf{M}_{\mathbf{m}}+\tilde{\theta} \mathbf{N}\right] \underline{u}^{n+1}+\theta \Delta t \mathbf{G}^{\mathbf{T}} \underline{p}^{n+1} } & =\left[\mathbf{M}_{\mathbf{m}}-\beta \theta \Delta t \mathbf{N}\right] \underline{u}^{n+1-\theta}+\theta \Delta t \mathbf{F}^{n+1}, \\
\mathbf{G} \underline{u}^{n+1} & =0,
\end{aligned}
$$

In this variable time stepping scheme other local time steps are chosen for the first and the third substep than for the second time step. In comparison with the first order implicit Euler scheme and Crank-Nicolson scheme the FS-scheme leads to about the same numerical complexity. With regard to stability of the FS-scheme detailed discussions can be found in the papers of Rannacher (1993), Rannacher et al. (1994) and Muller-Urbaniak (1993).

\subsection{Derivation of the Navier-Stokes solver}

The lack of an uncoupled equation for the pressure complicates the solution process for the Navier-Stokes equations. One way of overcoming this difficulty is to create a pressure field that satisfies the continuity equation. There is a large variety of such schemes which have been used in practice for several years. In this work a second-order accurate pressure correction scheme due to Van Kan (1986) is deployed.

In this scheme the treatment of incompressibility is taken into account by splitting the coupled problem of the Navier-Stokes equations and the continuity equation, and in so doing obtaining independent problems for the velocities and the pressure (Donea 1982, Turek 1998, Zienkiewicz et al. 2000).

The solution at each time step $\Delta t$ is obtained through three substeps: 
- Step 1: Calculation of the provisional velocities.

For convenience compact notation is used for the diffusive and convective parts, and we write

$$
N u_{j}:=u_{i} \frac{\partial u_{j}}{\partial x_{i}}+\frac{\partial}{\partial x_{i}}\left[\nu_{e f f}\left(\frac{\partial u_{i}}{\partial x_{j}}+\frac{\partial u_{j}}{\partial x_{i}}\right)\right]
$$

The provisional velocities ${u_{i}^{*}}^{n+\theta}$ are calculated from the linearized convectiondiffusion equation at time $t_{n+\theta}$, using the momentum equation; that is,

$$
[I+\theta N] u_{i}^{m^{n+\theta}}=\left[I-\theta \Delta t N\left(u_{i}^{n}\right)\right] u_{i}^{n}-\theta \Delta t \frac{\partial p^{n}}{\partial x_{i}}-\theta \Delta t\left(g \frac{\rho^{n}-\rho_{r}}{\rho_{r}}\right) \delta_{i 2} .
$$

The right hand side contains the gravity force and an "old" pressure approximation from the previous iteration at time $t_{n}$. Since the new velocities $u_{i}^{{ }^{n+\theta}}$ do not necessarily satisfy the continuity equation, they are called provisional velocities.

- Step 2: Solution of the pressure equation.

Next, an approximation to the pressure is obtained from (5.70): that is,

$$
u_{i}^{n+\theta}=u_{i}^{* n+\theta}-\theta \Delta t\left(\frac{\partial \Delta p}{\partial x_{i}}\right)^{n+\theta} .
$$

Differentiating 5.71 with respect to $x$ and $y$ for $i=1$ and 2 , respectively, then adding the two, and rearranging we obtain the Pressure-Poisson equation for $\Delta p$, that is,

$$
\left(\frac{\partial^{2} \Delta p}{\partial x_{i} \partial x_{i}}\right)^{n+\theta}=\frac{1}{\theta \Delta t} \frac{\partial u_{i}^{n+\theta}}{\partial x_{i}}
$$

In the above derivation $\left(\partial u_{i} / \partial x_{i}\right)^{n+\theta}$ has been equated to zero.

- Step 3: Velocity and pressure correction.

The new 'discretely divergence-free' velocity $u_{i}^{n+\theta}$ is corrected at time step $t_{n+\theta}$ by using (5.72) again, to obtain

$$
u_{i}^{n+\theta}=u_{i}^{* n+\theta}-\theta \Delta t\left(\frac{\partial \Delta p}{\partial x_{i}}\right)^{n+\theta},
$$

and the new pressure $p\left(t_{n+\theta}\right)$ is now

$$
p\left(t_{n+\theta}\right)=p\left(t_{n}\right)+\Delta p\left(t_{n+\theta}\right) .
$$


The above-mentioned steps are repeated until the approximations converge to an acceptable tolerance.

The discretized equations of this projection scheme written in matrix form are as follows:

Step1:

$$
[\mathbf{M}+\theta \widehat{\mathbf{N}}] \underline{u}^{n+\theta}=[\mathbf{M}-\theta \widehat{\mathbf{N}}] \underline{u}^{n}+\theta \Delta t \mathbf{G}^{\mathbf{T}} \underline{p}^{n}+\theta \Delta t \mathbf{F}^{n}
$$

Step2:

$$
\mathbf{H}(\Delta \underline{p})^{n+\theta}=\frac{1}{\theta \Delta t} \mathbf{G} \underline{u}^{n+\theta}
$$

Step3:

$$
\begin{gathered}
\underline{u}^{n+\theta}=\underline{u}^{n+\theta}-\mathbf{M}^{-1} \Delta t \mathrm{G}^{\mathrm{T}}(\Delta \underline{p})^{n+\theta}, \\
\underline{p}^{n+\theta}=\underline{p}^{n}+(\Delta \underline{p})^{n+\theta} .
\end{gathered}
$$

Here the matrices $\mathbf{G}, \mathbf{M}, \widehat{\mathbf{N}}$ and $\mathbf{F}$ are

$$
\begin{gathered}
\mathbf{G}=\int_{\Omega}(\nabla \mathbf{N})^{\mathbf{T}} \mathbf{N} d \Omega, \\
\mathbf{M}=\int_{\Omega} \mathbf{N}^{\mathbf{T}} \mathbf{N} d \Omega \\
\widehat{\mathbf{N}}=\int_{\Omega} \mathbf{N}^{\mathbf{T}}(\nabla \underline{u} \mathbf{N}) d \Omega+\int_{\Omega} \nu_{e f f}(\nabla \mathbf{N})^{\mathbf{T}} \nabla \mathbf{N} d \Omega, \\
\mathbf{F}=\int_{\Omega} \mathbf{N}^{\mathbf{T}}\left(g \frac{\rho-\rho_{r}}{\rho}\right) d \Omega+\int_{\Gamma} \mathbf{N}^{\mathbf{T}} \tau d \Gamma, \\
\mathbf{H}=\int_{\Omega}(\nabla \mathbf{N})^{\mathbf{T}} \nabla \mathbf{N} d \Omega .
\end{gathered}
$$

It should be noted that the same matrix $\mathbf{N}$ of basis functions is chosen for the pressure and velocity; this choice will be justified in the following subsection.

\subsubsection{Circumventing the Babuska-Brezzi restriction}

As mentioned earlier, many of the velocity-pressure elements, and in particular those with equal-order interpolation for velocity and pressure, do not satisfy the Babuskka-Brezzi condition. However, there is a mechanism by which the Babuska-Brezzi restriction can be circumvented. These algorithms exploit the time-dependent nature of the problem to construct a stable scheme that leads to convergent approximations in the steady limit (see Zienkiewicz \& Wu 1991). This 
very efficient method is implemented in the fluid mechanics algorithm named the characteristic based split (CBS) procedure recently developed by Zienkiewicz and co-researchers $(1995,1999)$.

Taking the three steps of the above described Van Kan's projection scheme and considering for simplicity here only the stationary Stokes problem we have, with $\Delta \underline{p}=0$

$$
\left[\begin{array}{cc}
\mathbf{K} & \mathbf{G}^{\mathrm{T}} \\
\mathbf{G} & 0
\end{array}\right]\left\{\begin{array}{c}
\underline{u} \\
\underline{p}
\end{array}\right\}=\left\{\begin{array}{c}
\mathbf{F} \\
0
\end{array}\right\}
$$

where

$$
\mathbf{K}=\int_{\Omega} \nu_{e f f}(\nabla \mathbf{N})^{\mathbf{T}} \nabla \mathbf{N} d \Omega .
$$

This system is not always positive-definite ${ }^{2}$ and can lead to singular solutions for certain choices of interpolation functions $N_{u}$ and $N_{p}$.

For Chorin's projection scheme the three steps as described earlier become

$$
\begin{gathered}
{[\mathbf{M}+\theta \mathbf{K}] \underline{u}^{* n+\theta}=[\mathbf{M}-\theta \mathbf{K}] \underline{u}^{n}+\theta \Delta t \mathbf{F}^{n},} \\
\mathbf{H}(\underline{p})^{n+\theta}=\frac{1}{\theta \Delta t} \mathbf{G} \underline{u}^{* n+\theta}, \\
\underline{u}^{n+\theta}=\underline{u}^{* n+\theta}-\mathbf{M}^{-1} \Delta t \mathbf{G}^{\mathbf{T}}(\underline{p})^{n+\theta} .
\end{gathered}
$$

In the steady state case we have

$$
\left[\begin{array}{cc}
\mathbf{K} & \mathbf{G}^{\mathbf{T}} \\
\mathbf{G} & \Delta t\left[\mathbf{G M}^{-1} \mathbf{G}^{\mathbf{T}}-\mathbf{H}\right]
\end{array}\right]\left\{\begin{array}{l}
\underline{u} \\
\underline{p}
\end{array}\right\}=\left\{\begin{array}{l}
\mathbf{F} \\
0
\end{array}\right\} .
$$

This system is now positive-definite and for any interpolation functions $N_{u}$ and $N_{p}$ a non-singular solution is obtained. In this way the Babuska-Brezzi restriction is circumvented (see Zienkiewicz \& Codina 1995, Zienkiewicz et al. 1995, 1999 and Zienkiewicz \& Taylor 2000).

In this work a combination of the two projection schemes is chosen in order to combine the better accuracy of Van Kan's projection scheme and its better star bility, with the property of Chorin's projection scheme of avoiding and correcting the checkerboard pressure. The implementation is described in Section 5.8.

\footnotetext{
${ }^{2} \mathrm{~A} n n \times n$ matrix $\mathbf{A}$ is said to be positive-definite if $\underline{u}^{T} \mathbf{A} \underline{u} \geq 0$ for all $n$-vectors $\underline{u}$, and if $\underline{u}^{\mathbf{T}} \mathbf{A} \underline{u}=0$ implies $\underline{u}=\mathbf{0}$.
} 


\subsection{Solution of systems of linear equations}

Methods for solving large sets of linear algebraic equations resulting from the finite element formulation can be divided into direct and indirect methods. The direct methods are based on the Gaussian elimination procedure, and an example is the LU decomposition. Gaussian elimination is liable to accumulate errors when the system is large and not sparse. Pivoting in order to obtain pivot elements as large as possible exacerbates the problem. Furthermore, it can result in very large storage and computation time, especially for three-dimensional problems.

Iterative methods, on the other hand, use successive approximations to systematically obtain improved solutions at each iteration step. If each iteration step is fast and the iteration number is small, an iterative solver is more efficient than the direct methods. This is usually the case in CFD.

In general there are two types of iterative methods, namely, the stationary and the nonstationary methods. Stationary methods, such as Jacobi, Gauss-Seidel and the successive overrelaxation method (SOR), are easier to understand and to implement, but mostly not as efficient as nonstationary methods.

Nonstationary iteration methods are a quite recent development (Barrett et al. 1994, Kelly 1995). In contrast to the stationary methods the computations involve information that changes at each iteration. This makes these methods more complex, but on the other hand they can be very effective. In this work two nonstationary iteration methods are used exclusively to solve the system of equations: the Conjugate Gradient (CG) method for the symmetric Poisson equation for the pressure, and the Generalized Minimal Residual (GMRES) method to solve the non-symmetric systems. CG and GMRES are often called matrix-free, as the global matrix does not need to be formed or stored; only a routine for matrix-vector products is required. Furthermore, CG and GMRES are attractive as they provide a reference for checking the convergence of the inner iterations. At the same time they provide a measure of the convergence of the outer iterations.

The following subsections give some insight to these methods. First, it will be necessary to give a brief overview of the Gram-Schmidt orthogonalization. 

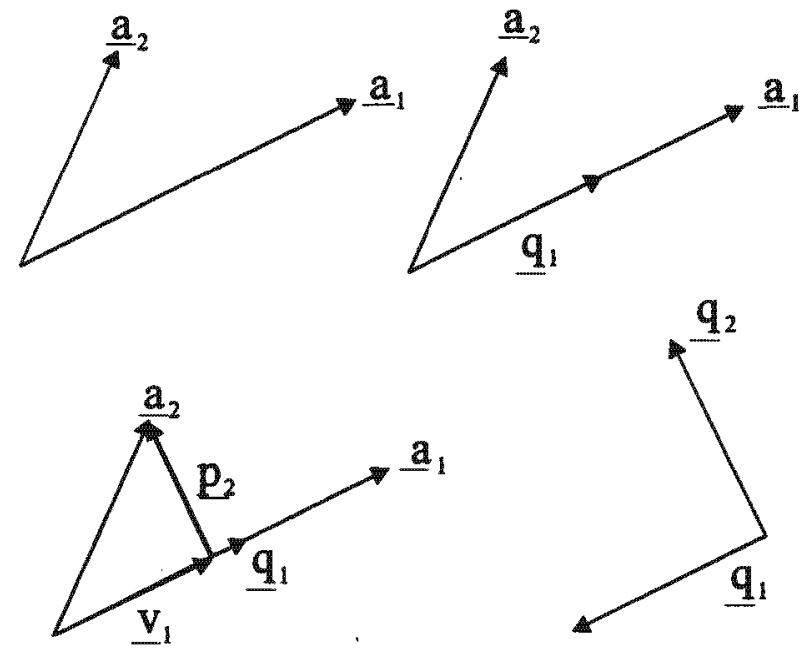

Figure 5.6: The Gram-Schmidt process.

\subsubsection{Gram-Schmidt orthogonalization}

The Gram-Schmidt process is a method for computing an orthonormal basis from a arbitrary set of linearly independent vectors $\left[\underline{a}_{1}, \underline{a}_{2}, \ldots, \underline{a}_{n}\right]$.

As illustrated in Figure 5.6 it starts by normalizing $\underline{a}_{1}: \underline{q}_{1}=\underline{a}_{1} /\left\|\underline{a}_{1}\right\|_{2}$. The vector $\underline{a}_{2}$ will then be expressed as the sum of a vector $\underline{v}_{2}$ in the direction and a vector $\underline{p}_{2}$ that is orthogonal to $\underline{a}_{1}: \underline{a}_{2}=\underline{v}_{2}+\underline{p}_{2}$. The vector $\underline{v}_{2}$, which is a projection of $\underline{a}_{2}$ onto is $\underline{q}_{1}$, is $\underline{v}_{2}=\left(\underline{q}_{1}^{T} \underline{a}_{2}\right) \underline{q}_{1}$. The vector $\underline{p}_{2}=\underline{a}_{2}-\left(\underline{q}_{1}^{T} \underline{a}_{2}\right) \underline{q}_{1}$ is then normalized to obtain $\underline{q}_{2}$ :

$$
\underline{q}_{2}=\frac{\underline{p}_{2}}{\left\|\underline{p}_{2}\right\|_{2}}=\frac{\underline{a}_{2}-\left(\underline{q}_{1}^{T} \underline{a}_{2}\right) \underline{q}_{1}}{\left\|\underline{a}_{2}-\left(\underline{q}_{1}^{T} \underline{a}_{2}\right) \underline{q}_{1}\right\|_{2}} .
$$

Thus $\underline{q}_{2}$ is a unit vector and is orthogonal to $\underline{q}_{1}$. In general, $\underline{p}_{j}$ can be expressed as $\underline{p}_{j}=\underline{a}_{j}-\sum_{i=1}^{j-1}\left(\underline{q}_{i}^{T} \underline{a}_{j}\right) \underline{q}_{i}$, where $\underline{q}_{j}$ are the orthonormal vectors normalized by $\underline{p}_{j}: \underline{q}_{j}=\underline{p}_{j} /\left\|\underline{p}_{j}\right\|_{2}$. Letting $\underline{r}_{j j}$ denote $\left\|\underline{p}_{j}\right\|_{2}$ and $\underline{r}_{i j}=\underline{q}_{i}^{T} \underline{a}_{j}$ the vectors $\underline{q}_{j}$ and $\underline{p}_{i}$ can be written as

$$
\begin{aligned}
& \underline{q}_{j}=\frac{\underline{p}_{j}}{\underline{r}_{j j}} \\
& \underline{p}_{j}=\underline{r}_{j j} \underline{q}_{i}=\underline{a}_{j}-\sum_{i=1}^{j-1} \underline{r}_{i j} \underline{q}_{i} .
\end{aligned}
$$




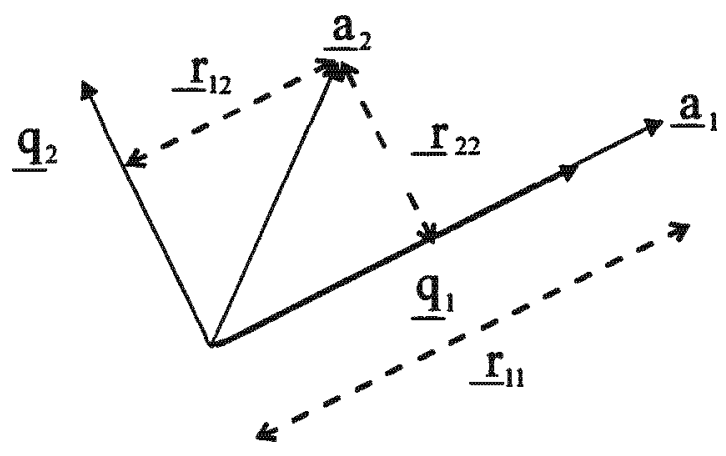

Figure 5.7: The Gram-Schmidt process for the case of two vectors.

Solving for $\underline{a}_{j}$, we have

$$
\underline{a}_{j}=\sum_{i=1}^{j} \underline{q}_{i} \underline{r}_{i j} \quad \text { or } A=Q R,
$$

where $A$ is the matrix whose columns are $\underline{a}_{i}, Q$ is the matrix whose columns are $\underline{q}_{i}$, and $R$ is the upper triangular matrix formed from the $\underline{\tau}_{i j}$. The Gram-Schmidt process is illustrated in Figure 5.9 for the case of two vectors.

The classical Gram-Schmidt method may be modified by replacing $r_{i j}$ with the matrix $\tilde{r}_{i j}=q_{i} q_{j}$. This leads to a more accurate scheme later.

\subsubsection{The Conjugate Gradient (CG) Method}

The Conjugate Gradient (CG) method was first conceived in the 1950s (Hestenes \& Steifel 1952) and has come to enjoy wide use in the last 20 years. The CG method is used here to solve the symmetric positive definite system, that is, the Pressure-Poisson equation. The CG proceeds by generating successive approximations to the solution. Two coupled recurrences are used, one that updates residuals using a search direction vector, and one that updates the search direction with a newly computed residual.

The method is described here for the linear equation $A x=b$, where $A$ is a nonsingular $N \times N$ matrix, $b \in R^{N}$, and $x$ is the unknown vector. The solution is denoted by $x^{*}=A^{-1} b \in R^{N}$ is to be found, and the residual $r$ is defined as $r=b-A x$. 
In general, the $k$ th iterate $x_{k}$ of CG minimizes

$$
\phi(x)=\frac{1}{2} x^{T} A x-x^{T} b
$$

and if the minimizer is $\tilde{x}$ then

$$
\nabla \phi(\tilde{x})=A \tilde{x}-b=0
$$

and $\tilde{x}=x^{*}$ is the solution. The same result would be obtained by minimizing $\left\|x^{T} A x-\left(x^{*}\right)^{T} A x^{*}\right\|=\left\|x-x^{*}\right\|_{A}$. With the help of a so-called search direction $p_{k+1} \neq 0$ the iterates and the residuals can be updated for some scalar $\alpha_{k+1}$ so that $r_{k}^{T} A^{-1} r_{k}$ will be minimized. The search direction will be then updated by the residuals with some scalar $\beta_{k+1}$, which ensures that $p_{k+1}=r_{k+1}$ and $A p_{k}=r_{k}$ are orthogonal. The factors are given by

$$
\begin{aligned}
\alpha & =\frac{\left\|r_{k}\right\|_{2}^{2}}{p_{k+1}^{T} A p_{K+1}} \\
\beta & =\frac{\left\|r_{k}\right\|_{2}^{2}}{\left\|r_{k-1}\right\|_{2}^{2}}
\end{aligned}
$$

The CG algorithm in full is given below:

Algorithm $\mathrm{CG}(x, b, A, \epsilon, k \max )$

1. $r=b-A x, \rho_{0}=\|r\|_{2}^{2}, k=1$.

2. Do While $\sqrt{\rho_{k-1}}>\epsilon\|r\|_{2}$ and $k<k \max$

a) if $k=1$ then $p=r$ else $\beta=\rho_{k-1} / \rho_{k-2}$ and $p=r+\beta p$ else $\beta=\rho_{k-1} / \rho_{k-2}$ and $p=r+\beta p$

b) $\quad w=A p$

c) $\quad \alpha=\rho_{k-1} / p^{T} w$

d) $x=x+\alpha p$

e) $\quad r=r-\alpha w$

f) $\rho_{k}=\|r\|_{2}^{2}$

g) $k=k+1$

The derivation of the CG minimization method is described in detail in Kelly (1995). 


\subsubsection{The generalized minimal residual (GMRES) method.}

The GMRES method is employed to solve the non-symmetric nonlinear system of the discrete momentum and concentration equations (Saad et al. 1986). The GMRES method is based on the modified Gram-Schmidt procedure. It generates a sequence of orthogonal vectors, which are the residuals of the iterates. But in the absence of symmetry the previously computed vectors in the orthogonal sequence have to be retained. To avoid large storage requirements and to minimize the work per iteration, the restarted version of GMRES $(m)$ is adopted, where $m$ is the number of restarts. The decision about when to restart is one of the critical components for successful application of this method.

The iterates of the GMRES are constructed as

$$
x_{k}=x_{0}+y^{1} v_{1}+\ldots+y^{k} v_{k} \text { or }
$$

where the coeffcient vector $y$ minimizes the least squares problem

$$
\underset{y}{\operatorname{minimize}}\left\|b-A\left(x+V_{k} y\right)\right\|_{2}=\left\|r_{0}-A V_{k} y\right\|_{2}
$$

and $V_{k}$ as the orthonormal basis. In order to create an orthonormal basis for the GMRES use is made of Gram-Schmidt orthogonalization. This orthogonalization is known as the Arnoldi process, and the algorithm is as follows:

Algorithm Arnoldi $\left(x_{0}, b, A, k, V\right)$

Define $r_{0}=b-A x_{0}, v_{1}=r_{0} /\|r\|_{2}$.

for $j=1$ to $k-1$

$$
v_{i+1}=\frac{\left.A v_{i}-\sum_{j=1}^{i}\left(A v_{i}\right)^{T} v_{j}\right) v_{j}}{\left.\| A v_{i}-\sum_{j=1}^{i}\left(A v_{i}\right)^{T} v_{j}\right) v_{j} \|_{2}}
$$

The inner product coefficients $\left.\sum_{j=1}^{i}\left(A v_{i}\right)^{T} v_{j}\right) v_{j}$ and $\left.\| A v_{i}-\sum_{j=1}^{i}\left(A v_{i}\right)^{T} v_{j}\right) v_{j} \|_{2}$ are stored in an upper Hessenberg matrix, and Givens rotation is used to reduce the Hessenberg matrix to triangular form, thereby solving the Hessenberg least squares problem. The derivation and implementation of the full GMRES method is desrcibed in Kelly (1995) and Barett et al. (1994). 


\subsection{Solving the full set of equations}

\subsubsection{Linearization}

The numerical scheme for discretizing the mathematical model requires the solution of systems of linear equations to solve for the nodal variables. The system is coupled and nonlinear. There are two approaches to solving such coupled systems: simultaneous, and sequential. In order to account for the strong interdependence of pressure, concentration and turbulent sizes the partitioning of the variables into the three subsets of velocity components, pressure and concentration, and of turbulent kinetic energy and its dissipation was made; that is, the system is decoupled through the use of an iterative scheme and through linearization of the system.

At every new time sub-step $\theta \Delta t$ the concentration equation predicts the new density $\rho$ of the fluid mixture, which is then used to update the gravity density term in the momentum equation for the vertical velocity $v$. The updated gravitydensity term will then yield a new velocity field, which again will influence the distribution of the concentration $c$. A SST with regions of different turbulence requires the use of the $k$ - $\varepsilon$-model. The predicted turbulent viscosity $\nu_{t}$ and diffusivity $\nu_{t} / \sigma_{s}$, respectively, can highly change the concentration and velocity field, which in turn may influence the turbulent values $k$ and $\varepsilon$.

Owing to this complexity the complete numerical solution process is split into two parts: the inner and the outer iterations. At each inner iteration the source terms and the coefficients that depend on the other variables are kept fixed. At the outer iterations the coefficient matrices and the source vector must be updated and the process repeated to obtain a solution that satisfies all of the equations. The right number of inner iterations per outer iteration has to be chosen carefully to optimize the solution process.

The nonlinear terms (convective flux, source term) are linearized using Picard iteration (Kelly 1995), so that the convective term $u_{i} \partial u_{j} / \partial x_{i}$ is

$$
u_{i}^{0} \frac{\partial u_{j}}{\partial x_{i}},
$$

where the index 0 indicates that the value is taken from the result of the previous outer iteration. 


\subsubsection{An overall view of the algorithm}

The same order of finite element interpolation was chosen for all variables $u, v, p, c$ as well as for $k$ and $\varepsilon$. This has the great advantage that nodal values of all variables are given at all nodes.

Flows in secondary settling tanks are very unstable due to the very small velocities and their design in general. The flow in the main section of the tank is highly influenced by gravity forces. Although the changes in the density of the fluid mixture are very small, the rate of momentum is of the same order and can therefore be influenced very easily by these 'small' gravity forces. The variable density of the fluid mixture, the hydrostatic pressure and the velocities are highly coupled: the variable density results in a hydrostatic pressure that strongly affects the velocities. Furthermore, flow and settling motion react very sensitively to the values of turbulent viscosity and diffusivity.

To take these sensitivities into account, the author decided not to use element with discontinuous pressure, such as the stable biquadratic velocity-linear discontinuous pressure quadrilateral element. For reasons of economy it was decided to use a four-noded bilinear quadrilateral for all variables. Although the constraint ratio of this element is with optimal $r=2$, it does not satisfy the Babuska-Brezzi condition, and it exhibits checkerboard pressure modes. However, these difficulties are overcome by using the stable approach described in Section 5.6.1. The scheme, based on Chorin's method, works well for neutral density flows, such as for the benchmark results presented in Chapter 6, but due its first order accuracy it shows instabilities when the hydrostatic pressure becomes an increasing influence, which is always the case in SSTs. Owing to the difficulties of Chorin's scheme, which occur with increasing hydrostatic pressure, the time step has to be very small. This would make an economical investigation of the long time behaviour of SSTs impossible. Van Kan's projection scheme, on the other hand, is of second-order accuracy and is stable even for very high concentrations (high hydrostatic pressure) and large time steps $(\Delta t=10 \mathrm{~s})$, but it exhibits spurious pressures. Even though this polluted pressure has no significant influence on the general flow pattern and sludge contribution, the pressure results are not meaningful.

For the successful development of the program NACOFEA (NAvier-Stokes and COncentration Finite Element Analysis) the author decided to combine the 
features of both schemes. Van Kan's projection scheme is mainly used for its accuracy and economy. Then, in order to smooth and correct the pressure Chorin's scheme is applied after a fixed number of iterations that depends on the respective flow problem in this step. In summary, Van Kan's projection scheme calculates the long time behaviour of the flow in the SSTs accurately, with the exception that it produces a checkerboard pressure and Chorin's projection scheme acts as a correction scheme in order to correct the pressure. The procedure is illustrated by the flow chart in Figure 5.8. In the following the construction of the program will be described in detail.

\subsubsection{The program}

Before presenting the details of the program NACOFEA some general remarks are necessary. As is common and well known in the area of CFD, convergence parameters are difficult to determine. In the special case of the program NACOFEA, these are the number of inner and outer iterations, the size of the mesh and macro time step $\Delta t$, the number of iterations within CG and GMRES, the number $m$ of restarts for the GRMES( $m)$, and the 'maximum errors' $\epsilon$ for each equation set to achieve maximum convergence and best results. The values of these convergence parameters strongly depend on the respective flow problem and they have to be chosen individually. Especially for the simulation of SSTs, they have to be selected very carefully owing to the very unstable and sensitive flow behaviour in these tanks and they differ from tank to tank. It has proved necessary to experiment with various values in order to find the best combination for the problem at hand.

In the following the program NACOFEA will be described in more detail, using also the flow chart in Figure 5.8:

Starting with an initial guess $u_{o}, v_{o}$ and $p_{o}$ for the velocities and pressure and a small time step $\Delta t$ the flow field of the neutral density case is calculated. During the iterations the time step $\Delta t$ be increased continuously up to the larger time step such that the tolerance $\epsilon$ is not exceeded. The prediction of the unknowns is achieved using Van Kan's projection scheme. After a fixed number of iterations depending on the problem and on the chosen time step, the checkerboard pressure field is corrected with Chorin's projection scheme, using a very small time step $t \approx 0.1 \mathrm{~s}$. In a single time step the polluted pressure can be smoothed 
and rectified. A maximum number of inner iterations IIMAX is chosen for the calculation of the momentum equations and the loop over the projection schemes.

In the case of variable turbulence the prediction of the kinetic energy $k$ and its dissipation $\varepsilon$ are added to the algorithm, based on the already predicted flow field and on initial values for $k_{o}$ and $\varepsilon_{o}$. The turbulent viscosity $\nu_{t}$ is updated for the next iteration loop over the projection scheme to calculate the new velocity and pressure field.

After a steady state solution is reached the actual simulation of the SST will begin by calculating the scalar equation for the activated sludge concentration $c$, setting the initial values of $c_{o}$ to nearly zero and adjusting the diffusivity to $\left(\nu_{t}+\nu_{l}\right) / \sigma_{S}$ when variable turbulence is required. After every time sub-step $\theta \Delta t$ the density $\rho$ of the fluid mixture will be predicted to update the gravity-density term in the momentum equation of the vertical velocity $v$. A new loop over the projection scheme follows with a new density $\rho$ and with the updated turbulent viscosity $\nu_{t}$. After convergence is reached to within an acceptable tolerance in the time sub-step, the next iteration cycle over the next time sub-interval will start. If the steady state solution is not reached after a complete loop over a macro time step $\Delta t_{n}$, a new calculation loop will follow for the next macro time step $\Delta t_{n+1}$. 


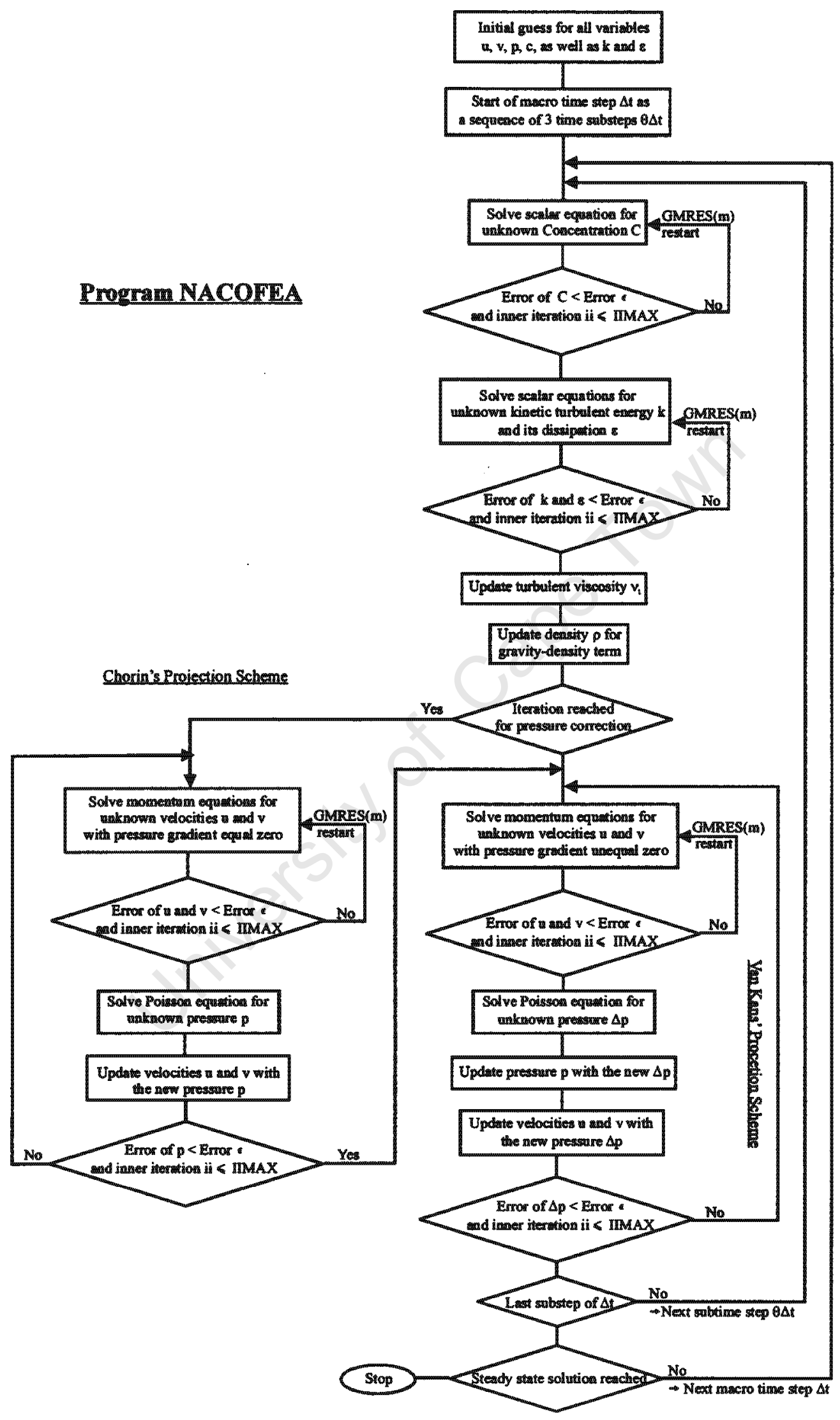

Figure 5.8: Flow chart for finite element code NACOFEA. 


\subsubsection{Errors and tolerances}

As described in Section 5.7 the CG and GMRES methods generate a sequence of orthogonal vectors, which are the residuals of the iterates and which provide a reference for checking the convergence. The solution of the linearized algebraic equations is considered to be converged when the residual norm is smaller than a specified proportion $\epsilon_{r}$ is of the initial residual, that is,

$$
\frac{\sum\left|r_{k+1}\right|}{\sum\left|r_{o}\right|} \leq \epsilon_{r}
$$

As already mentioned at the beginning of this section, the maximum tolerance $\epsilon_{r}$ for best convergence depends on the equation set and on the convergence parameters. A large time step, for example, will result in a large residual $r_{o}$, hence the value of $\epsilon_{r}$ has to be adjusted to the respective time step size. However, in order to give an idea of the quantity of these sizes, the amount of iterations is limited to 100 for the CG method, and the number of restarts $m$ for the $\operatorname{GMRES}(m)$ is restricted to 3 with maximal 20 inner iterations, otherwise loss of orthogonality will appear and divergence will occur. The inner iterations IIMAX lie in the range 2 to 6 and the residual norm $\epsilon_{r}$ should be around $10^{-3}$ depending on the flow problem and the variable. The convergence tolerances are adjusted in that manner such that convergence is reached after every sub-step, hence the number of outer iterations is three. The maximum macro time step $\Delta t$, for which convergence can still be attained, lies in the range of 5 to $10 \mathrm{~s}$. 


\section{Chapter 6}

\section{Numerical evaluation}

The modelling approach, algorithm and computer program developed in the previous chapters are presented here in two sections. In the first section the FEM code is applied to the typical and well-documented benchmark problems in order to assess the neutral-density flow.

The second section deals with the actual simulation of SST performance. The validation of the developed code is analysed with experimental data obtained through measurements on real secondary settling tanks. Additionally, the computational results of the FEM program are compared with the simulation results of the 2D hydrodynamic SST simulation package SettlerCAD (1998), which is based on the finite difference method. 


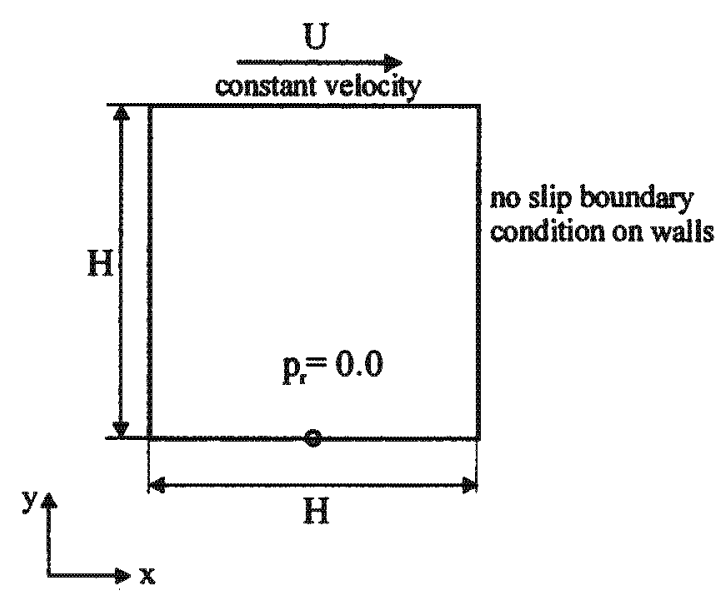

Figure 6.1: Geometry and boundary conditions for the lid-driven cavity flow problem.

\subsection{Benchmark results for the neutral density case}

In this section the finite element algorithm is assessed in context of three benchmark problems: lid-driven cavity flow, the flow over a backward-facing step, and the flow between two parallel plates. Simulation results are presented at the end of this section.

\subsubsection{Lid-driven cavity}

The classical lid-driven cavity flow problem is a standard test for validating CFD algorithms, and there is extensive documented data available for comparison. The problem has been studied by many researchers, and probably the most detailed investigation was that of Ghia et al. $(1982,1989)$, who presented many solutions and data for different Reynolds number.

The domain is a square of unit side. The velocity is zero on three of the sides, while along the side $y=H$ a constant velocity $\underline{u}=(U, 0)$ is prescribed. (Figure 6.1). A the corners $(0, H)$ and $(H, H)$ the velocity is set equal to zero, and at the nodes along $y=H$ that are adjacent to the corners the horizontal velocity is set equal to $U / 2$.

The Reynolds number is based on the lid velocity $U$ and on the domain side 
length $H$. Numerical computation for different Reynolds numbers $(R e=100,400,1000)$ were achieved by adjusting the kinematic viscosity $\nu$. In the present study a $50 \times 50$ mesh was used.

Figure 6.4 shows the computed velocity field, while Figure 6.5 gives the contours of the velocities, $u / U$ and $v / U$, respectively, as well as the pressure $p$.

It is seen that the recirculation zone shrinks as the Reynolds number increases. The zero contour of the vertical velocity $v / U$ remains close to the vertical geometric centre line. The location of the minimum pressure $p$ descends and shifts towards the geometric centre of the cavity with increase in Reynolds number.

Figure 6.6 shows the computed horizontal velocity profiles $u / U_{r}$ along the vertical line $x=H / 2$. Figure 6.7 gives the results for the same problem obtained by Ghia et al. $(1982,1989)$. The present results are in very good agreement with those of Ghia et al.

\subsubsection{Backward-facing step}

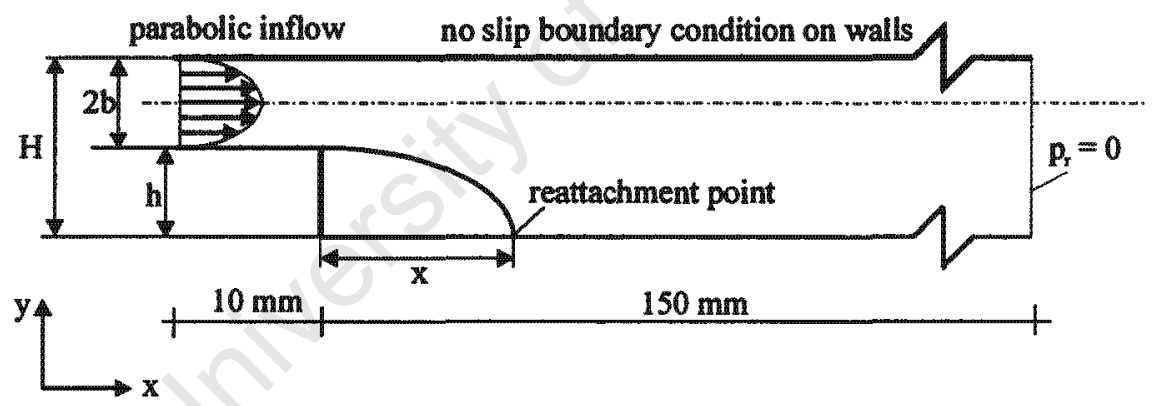

Figure 6.2: Geometry and boundary conditions for the backward facing step flow.

Armaly et al. (1983) used Laser-Doppler anemometry to measure experimentally the velocity distribution and reattachment length downstream of a backwardfacing step. They presented results for laminar, transitional and turbulent flow of air with Reynolds numbers in the range $70<R e<8000$. The channel height upstream of the step is $5.2 \mathrm{~mm}$ and the downstream channel height $H 10.1 \mathrm{~mm}$, which gives an expansion ratio $H / h=1.9423$ and a step height $h$ of $4.9 \mathrm{~mm}$. Figure 6.2 shows the geometry and boundary conditions for the backward-facing step flow. 
A $10 \times 10$ mesh was used upstream of the step, while downstream a $20 \times 114$ mesh was used. The velocity boundary conditions include no-slip conditions at all impermeable walls, a prescribed fully developed standard parabolic velocity profile at the upstream inflow, which can be written in terms of the average velocity $\bar{u}$. Along the outflow the reference pressure $p_{r}$ is set equal to zero.

The Reynolds number is based on the average bulk velocity in the upstream channel and the reference length is the hydraulic diameter of the upstream channel, which is defined as the twice the upstream channel height. The Reynolds numbers $(R e=400,800$ ) in the computation were adjusted by the kinematic viscosity $\nu$, while the reference velocity and the length scales were fixed. The predicted velocity patterns up to a length of $x / h=20$ are given in Figure 6.8 for the Reynolds number 400 and 800 .

In Figure 6.9 the primary reattachment lengths, normalized by the step height $h$, are plotted as a function of the Reynolds number.

For values of $R e$ up to approximately 400 , good agreement with the experimental values was achieved by various researchers with their computational results (see Figure 6.9), while they deviated from the experimental data for $R e>800$. The divergence is probably caused due to three-dimensional effects at high Reynolds numbers, as discussed in Armaly et al. (1982) and Williams \& Baker (1997). The three-dimensional simulations of Williams \& Baker (1997) showed very good agreement with the experimental results for $R e$ up to around 800 . The present computation gives good results for a Reynolds number of 400 , but for $R e=800$ it underpredicts the reattachment length by around $15 \%$. Thus the present results conform to the two-dimensional computations of other investigators, such as Gartling (1990).

\subsubsection{Flow between two parallel plates}

This example shown in Figure 6.3, is a good test for the existence of spurious pressure modes. The closed form solution for the horizontal velocity, the dynamic viscosity $\mu$ and the length of the channel length $L$, as well as the width $2 b$ between the two plates, and is given by

$$
u(y)=\frac{1}{2} \frac{\Delta p}{\mu L}\left(2 \frac{y}{b}-\frac{y^{2}}{b^{2}}\right)
$$




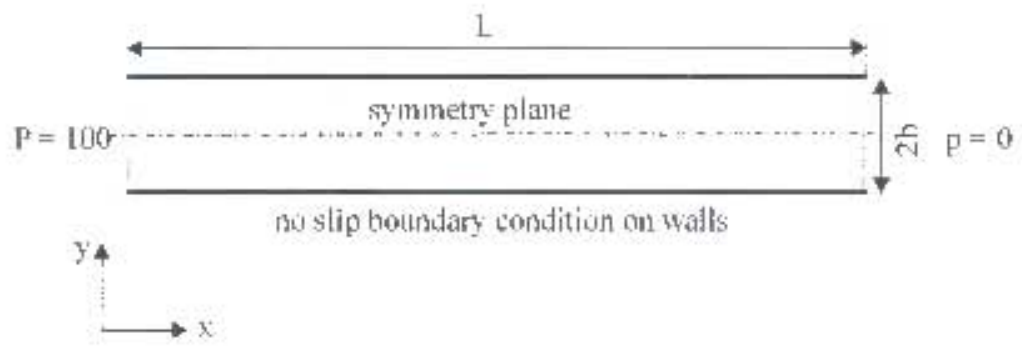

rigire 6.3: Geomedry and boundary conditions for the flow between two parallel plates.

whore $\Delta p$ is the pressure drop, $y$ is the vertical distance from the symuntry line. This can also be expressed in the alternative form

$$
u(y) \quad \frac{3}{2} u\left(2 \frac{y}{b}-\frac{y^{2}}{b^{2}}\right) \text {. }
$$

where $\bar{u}$ is the average velocity. The Reynolds number for the problem is based on the channe] width $2 b$ and the ancrage velocity ${ }^{2}$ wes: and is

$$
R e=\frac{p u_{a v g}(2 b)}{\mu} \text {. }
$$

Only hatf of the region betwen the plates newds to be discretized as the problem is syumetric. The mesh adopted contained $5 \times 250$ cements in the $x$ - and $y$ directions, respectively. Ihe present computalion predicts the velecity profile (6.1) exactly. The velocity pattern and the pressure contours for the dirveloping flow between the two parallel slabs are given in Figure 6.10. It is clearly obsorved that no spurious pressure modes exist.

\subsubsection{Results}
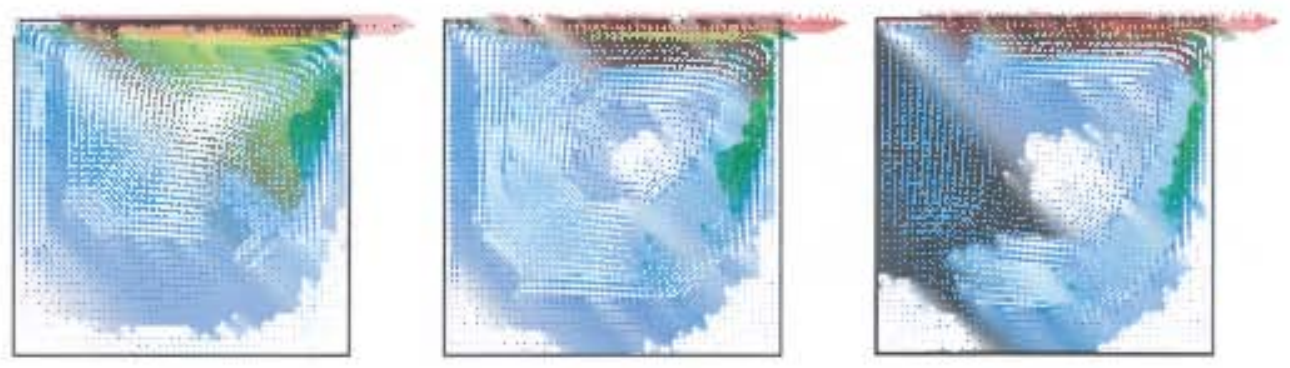

Figure 6.4: Flow patern of the lid-driven cavity flow problem for different Reynolds numbers: Re $=100,400$, and 1000 . 

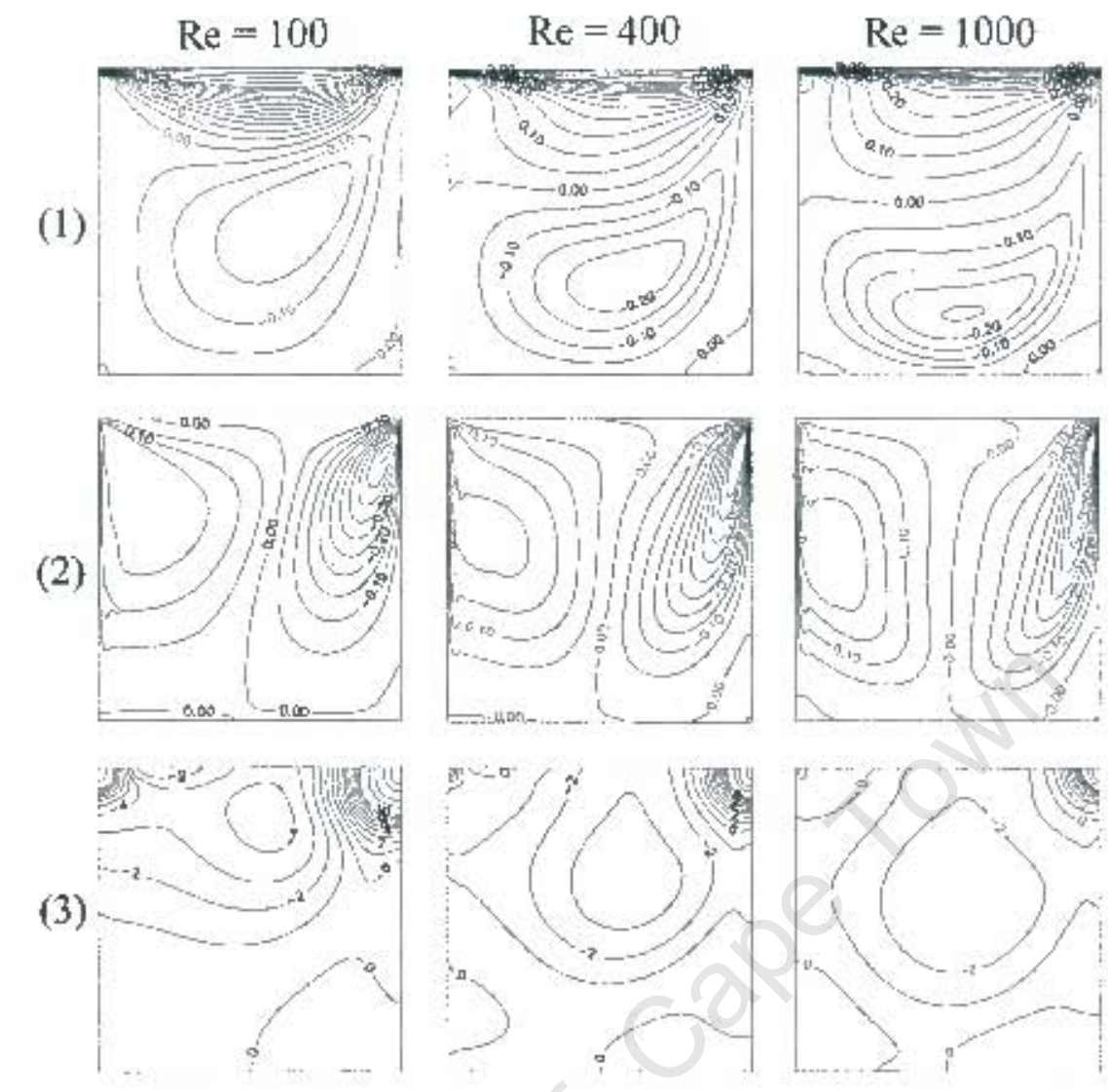

Figure 6.5: Computed results at Reynolds ntimbers $R e=100,400$ and 1000: (1) contours of the horizontal velocity $u / U ;(2)$ contours of the vertical velocity $v / U ;$ (3) pressure $p$ contours. 
lid-driven Cavity - Present study

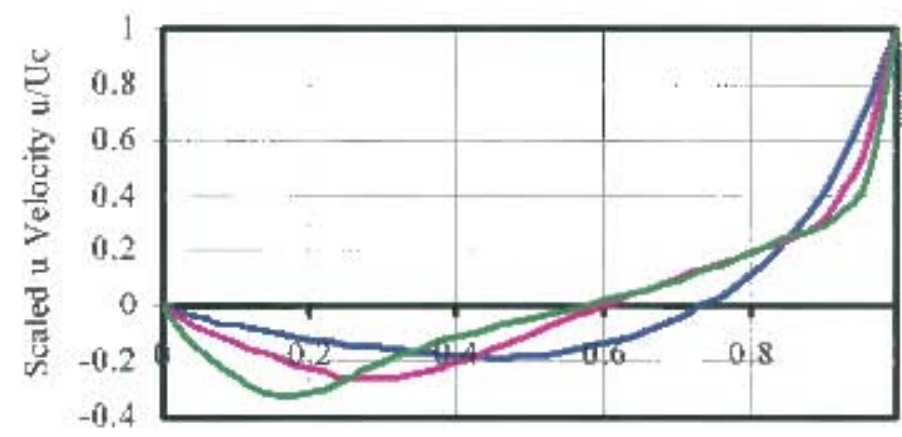

Vertical Distance y/H

$\longrightarrow \mathrm{Re}=100-\mathrm{Re}=400-\mathrm{Re}=1000$

Figure 6.6: Profile of the horizontal velocity $u / U$ along the line $x / H$ for Reynolds numbers $B e=100,400$ and 1000 .

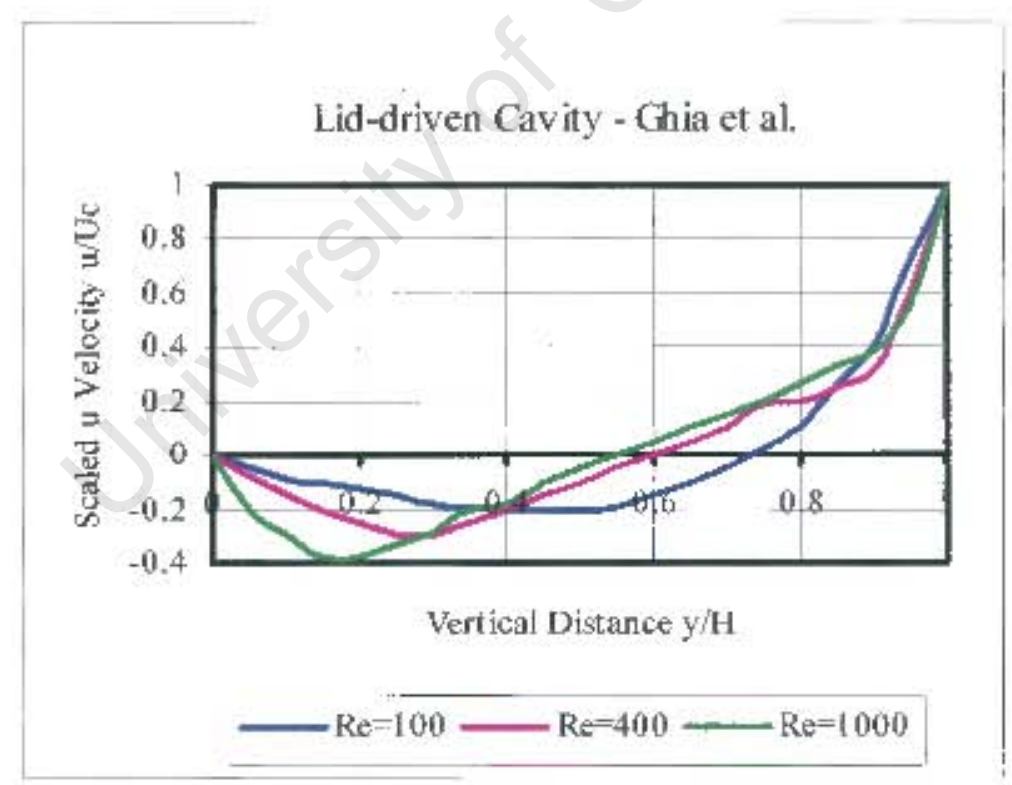

Figure 6.7: Profile of the horizontal velocity $u / U$ along the vertical line $x / H$ for Reynolds numbers $R e=100,400$ and 1000 computed by Ghia et al. (1982, 1989). 


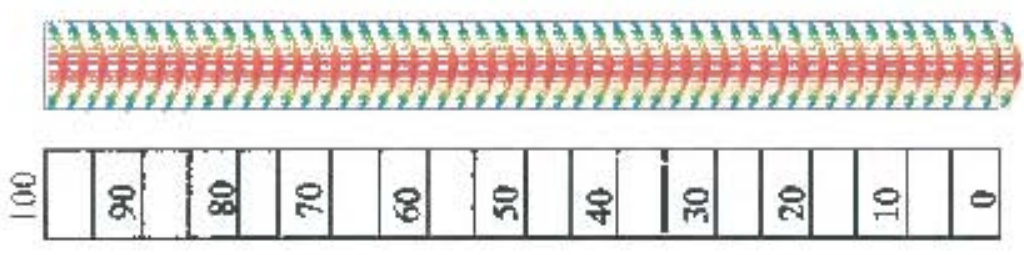

Figure 6.10: Flow pattern and pressure contours.

\subsection{Simulation of flows in SSTs}

The Inmoritul Inotel developed and described in earlier chapters is used in this chapter to simulate: flows in is varioty of sectondary settling tanks. Numerical rosullsis arc corwpared against tests carried out by De Haas et al. (1998) on SSTs at the Dirvill wistemater treatment plant, Pietermaritzburg. South Africa, as woll as strosis tostin by Wal.ts et. al. (1996) on one of the four SSTs at the Kanapaha Water Reclarration Fatility (Florida, USA). The numerical results are compared with the simmlation results of Ekama \& Marais (2002), who made use of the 2D hydrodynamic SST sinulation package SettlerCAD ( Zhou et al. 1998).

Ekana \& Marais (2002) exaluated the capatity of fill scale SSTs in relation to the idcalizod 1D flux theory with the CFD model SettlerCAI). Using the SST slirss tist. results observed by De Hass et al. (1998) on $2 \times 35 \mathrm{~mm}$ diameter 5STs, onc old $2.5 \mathrm{~m}$ deep and the other new $4.1 \mathrm{~m}$ deep at Darvill waste water troattrent. plant, Pietermaritzburg, South Africa, and by Watts et a.. (1996) on a $29 m$ diancher. $3.66 \mathrm{~m}$ deep SST at the Kanapaha Water Reclamation Fatility (Florida, USA). They calculated with SettlerCAD the S5T capacity as $\%$ of the 1DFT predicted maximum hytranlic loading ratle (HLR, (m/h) and solids loading ratks (SLR. kgSS $/\left(m^{2} h\right)$ ). Failure of the SST was defined as ESS $>50 \mathrm{mg} / \mathrm{l}$ and raised sludge blanket up to the effnent launders; the $r_{2}$ value in Takács sludge settling volocity explation was set. 1.o a very high value (10), making it equivalent to the Vesilind's equation (2.2) in Section 2.4, resultiug in very fast sct.ling velocity for low concentrations $(<100 \mathrm{mg} / \ell)$, so that SST failure would not misinterpreted from a high FSS wilhout a rasioxl siludge blanket. This was also done in this investigation. De Hass el al. (1998) conducted 4 siress tests on the old and new Darvill SSTs and Watts et al. (1996) 15 stress texsts on a single SST. The tests selected for simmlation wre thosc that were close to the SST cautcity as indicated both by observation and simulation with SettlerCAD 
(Ekama \& Marais 2002) .

To take turbulence effects into account two different models were used. A constant turbulent viscosity was chosen for the Darvill old and new tanks. This assumption is appropriate due to the fact that the turbulent inlet sections of both tanks are small compared with the settling section of the tank (Krebs, 1989). This is not the case for the Watts tank. The special construction of this SST makes it impossible to use a constant turbulent viscosity. The location of the skirt baffle divides the Watts tank into two main sections of similar size: an inlet section of high turbulence, which acts as a mixing and flocculating zone, and a section of low turbulence where settling takes place. Hence the turbulence of the inlet section and its influence on the second section cannot be neglected. The $k-\varepsilon$ model was used in the simulation of the Watts tank to take the different turbulence regions and their interaction into account. For the modelling of the diffusion coefficient in the concentration equation it was found that the best results were achieved by using Schmidt numbers larger than 1 . Additional information would be required to determine the extent to which the concentration distribution is affected by diffusion.

It should be borne in mind that the model used here does not include the effects of sludge compaction. These stress tests with extreme loading conditions cannot be simulated by the numerical model.

In the following section the geometry and the boundary conditions of the Darvill old and new SSTs, as well as those of the Watts' SST, are described. The specific boundary conditions, parameters and results of the simulations are presented at the end of this chapter.

\subsubsection{Geometry and boundary conditions}

The geometry and the boundaries of the simplified circular Darvill old and new tank, as well as for the Watts tank are shown in Figure 6.11.

Both Darvill tanks are circular, with a $35 \mathrm{~m}$ diameter. The side water depths of the old tank and new tank are $2.5 \mathrm{~m}$ and $4.1 \mathrm{~m}$, respectively. The old tank has a flat floor and six suction lifts for the settled sludge, while the new tank has a $10 \%$ sloping floor, and the sludge is scraped to a central hopper. 


\section{Darvill old tank}

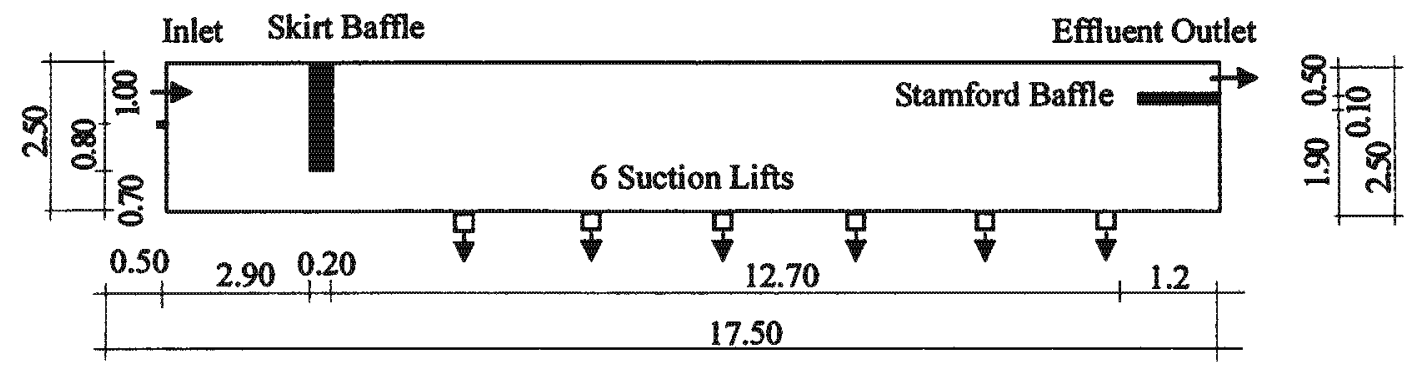

Darvill new tank
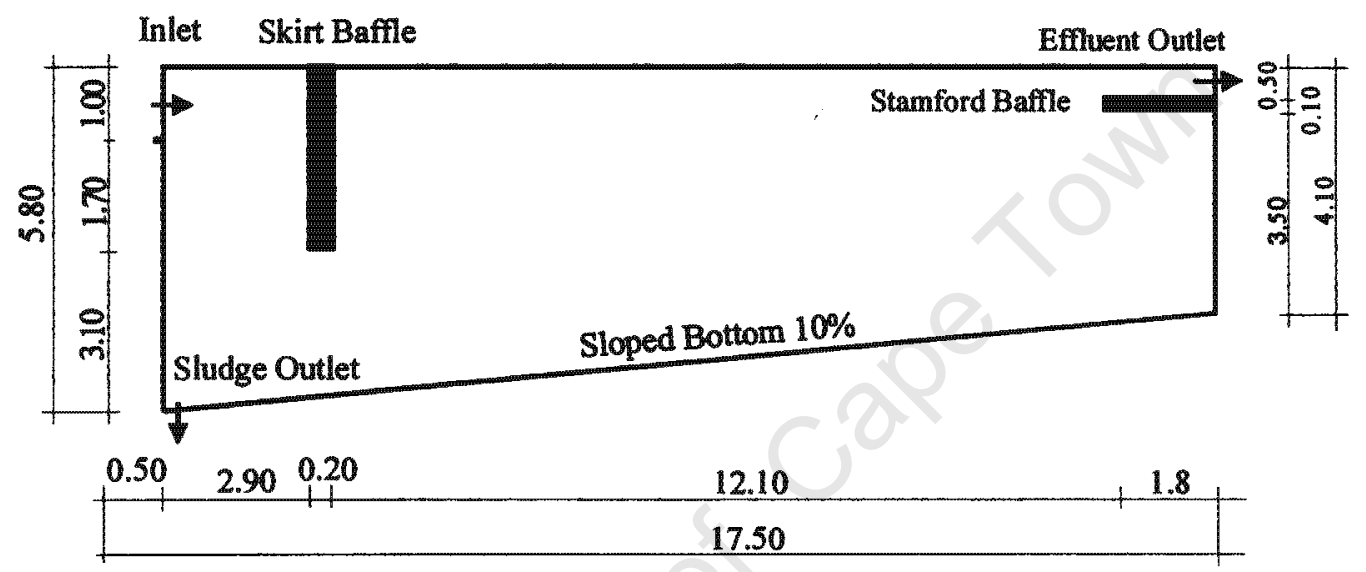

Watts tank
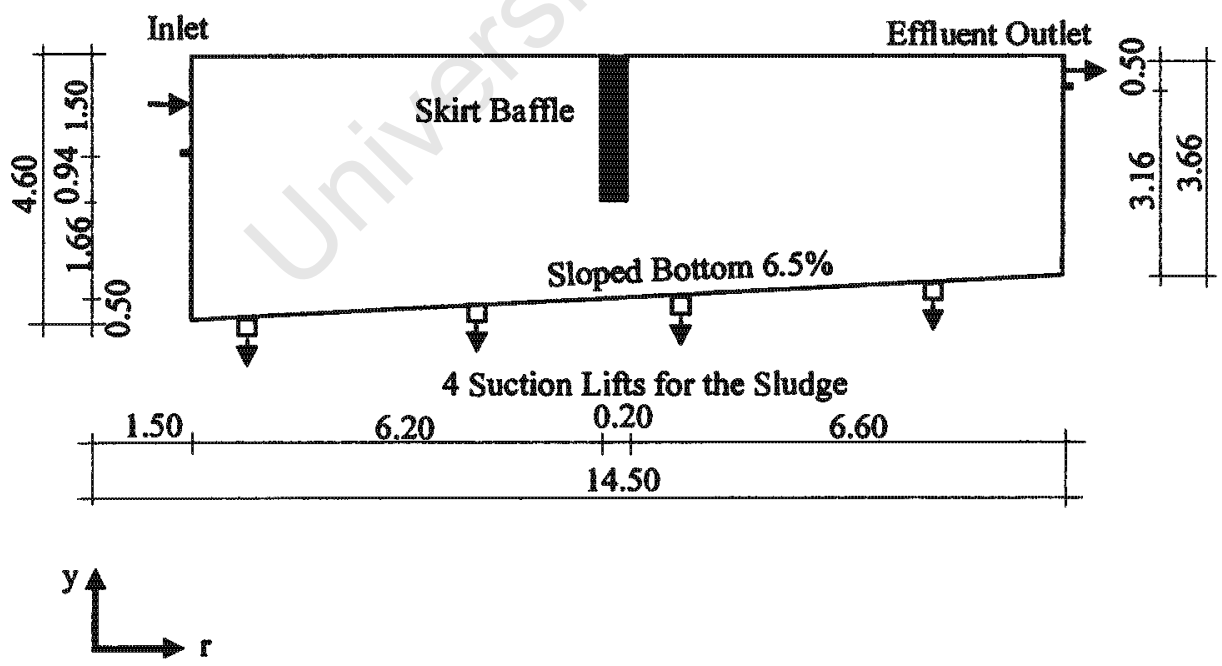

Figure 6.11: Geometry and boundaries for Darvill old and new tank, as well for the Watts SST. 
A $6.0 \mathrm{~m}$ diameter skirt baffle acts as flocculator at the centre well to water depth of $1.8 \mathrm{~m}$ and $2.7 \mathrm{~m}$, respectively. A peripheral Stamford baffle at the effuent outlet extends $1.2 \mathrm{~m}$ and $1.7 \mathrm{~m}$ from the side wall, respectively.

The circular Watts tank has a $29 \mathrm{~m}$ diameter and water depth of $3.66 \mathrm{~m}$, with a centre feed, peripheral and radial effluent overflows, and rotating multiple suction pipe sludge collection system (Figure 6.11).

To complete the mathematical description for the model the boundary conditions (BC) for the unknown variables have to be set. These are of two types:

1. essential (Dirichlet) $B C s$, where the value of the variable is prescribed;

2. natural (Neumann) BCs, where the first derivative is prescribed.

In the following the boundary conditions applicable to the tanks are described. In Figure 6.12 the boundaries of the tanks are shown. There are six parts to the boundaries: the inflow, effluent outlet, the outlet for the thickened sludge, the water surface, the bottom of the tank, and the rigid walls. At the end of this chapter the specific boundary conditions and results of the simulations, respectively, are listed in Tables 6.1 and 6.2 for the Darvill tanks, as well as in Tables 6.2 and 6.4 for the Watts tank.

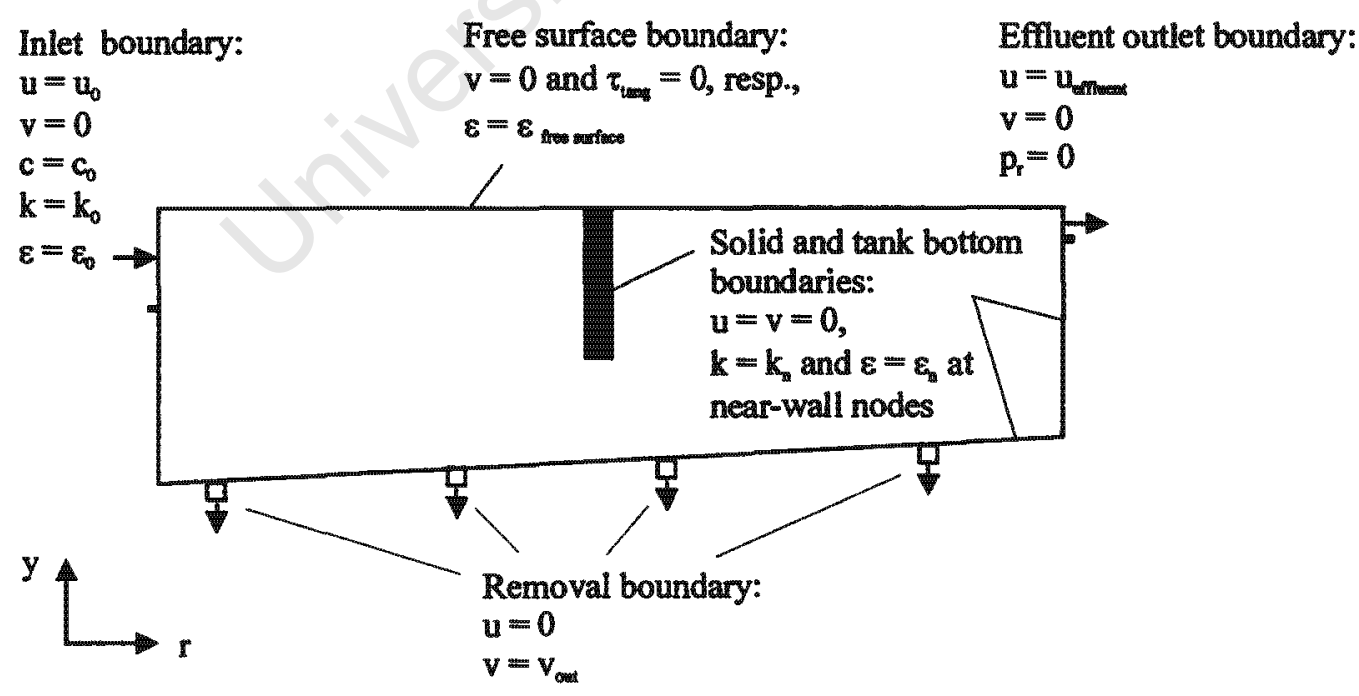

Figure 6.12: Boundary conditions for the simulation of SSTs. 


\section{(1) Inlet boundary}

In the model the inlet condition is idealized. The values of the variables $u, v, c, k$ and $\varepsilon$ are assumed to be uniformly distributed at the inflow boundary. $\mathrm{A}$ uniform horizontal inflow is imposed; that is, the radial velocity $u=u_{o}$ (constant), the vertical velocity $v=0$, and the inlet concentration $c=c_{o}$. The turbulent kinetic energy $k$ is related to the inlet kinetic energy by $k_{o}=K u_{o}^{2}$, where $K$ is chosen to be 0.2 (Ekama et al. 1997). The inlet dissipation rate is approximated assuming equilibrium of turbulence production and dissipation, and is given by

$$
\varepsilon_{o}=C_{\mu}^{3 / 4} \frac{k^{3 / 2}}{l_{m}},
$$

where the mixing length $l_{m}=C_{\mu}\left(0.5 H_{o}\right), C_{\mu}=0.09$, and $H_{o}$ is the water depth of the settling tank inlet.

\section{(2) Effluent outlet boundary}

The natural boundary conditions, which prescribe the gradients or fluxes, are zero at the boundary of the effluent outlet. In order to have a well-posed problem the reference pressure is set to zero at the effluent outlet. The baffles and the effluent weir are treated as a reflecting boundary, where the natural boundary conditions of the sludge concentration $c$ are zero. For the Watts tank the outlet velocities are prescribed with $v_{\text {out }}=0$, and $u_{\text {out }}$ is set to the value required to ensure mass balance over the entire tank. At one node at the outlet the reference pressure is set to zero.

\section{(3) Removal boundary}

The velocity normal to the bottom is equal to the return activated sludge flow divided by the removal outlet area affected, that is the outlet surface of the 6 and 4 suction pipes of the Darvill old and Watts tank, respectively, as well as the outlet surface of the hopper of the Darvill new tank. Due to the necessary simplification of 2D models the rotating multiple suction pipe sludge collection system sludge in the Darvill old tank and Watts tank is modelled by a continuously sludge withdrawal. The scraper of the Darvill new tank, which exerts a shear force, is not included in the model.

(4) Bottom and water surface boundary conditions for the sludge concentration

At the bottom of the tank and at the water surface additional natural boundary 


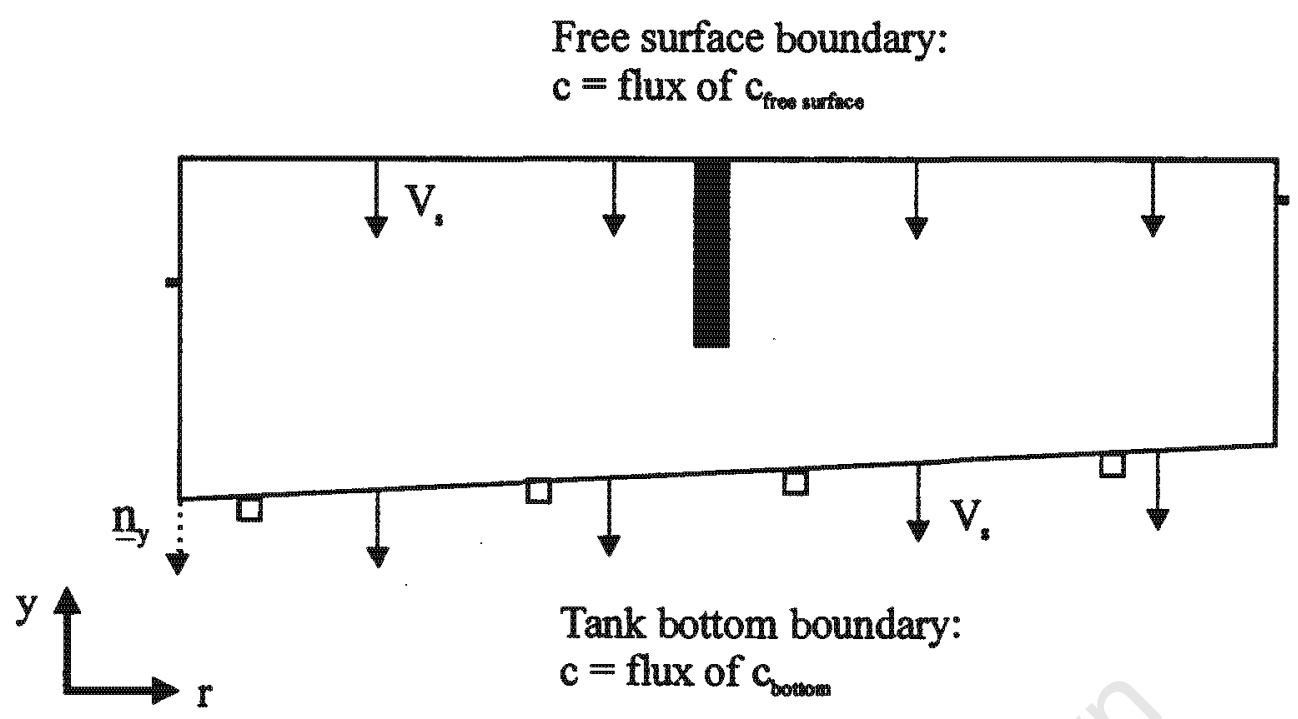

Figure 6.13: Flux boundary condition for the sludge concentration at the free surface and at the bottom of the tank.

conditions are necessary. The settling motion of the suspended solids is described by Vesilind's equation (2.2). For the prediction of the solid phase the settling velocity $V_{s}$ is added to the vertical velocity. As a result there are vertical velocities at the bottom of the tank and at the water surface, as illustrated in Figure 6.13. This corresponds to convective mass flux over the boundary of the domain. At the water surface there is a mass flux in the tank and at the bottom a mass flux out of the tank. In order to avoid this mass flux, an additional boundary condition has to be set. With that the sum of the convective and the mass transport due to diffusion must be zero. Taking the surface integral (5.26) of the discretized suspended solids equation (5.25), which is a line integral for the twodimensional case, the concentration gradient times the diffusion coefficient must be equal the settling velocity times the concentration, as proposed in Ekama et al. (1997), that is

$$
\begin{aligned}
& n_{y} \frac{\nu_{e f f}}{\sigma_{s y}} \frac{\partial c}{\partial y}=-V_{s} c \quad \text { at the water surface, } \\
& n_{y} \frac{\nu_{e f f}}{\sigma_{s y}} \frac{\partial c}{\partial y}=V_{s} c \text { at the bottom of the tank. }
\end{aligned}
$$

Here $n_{y}$ is the unit outward normal at the bottom and at the surface of the tank, as illustrated in Figure 6.13.

(5) Free surface boundary 
At the water surface, the rigid-lid approximation is made: this assumes that there is negligible change in water surface elevation over the tank. Gradients and fluxes, except for the concentration as explained above, perpendicular to the boundary are zero. The vertical velocity component is $v=0$ and the horizontal component $u$ is assuming full slip, that is no shear stress at the surface. The normal gradients of $u$ and of the kinetic energy $k$ are equal zero, and for the dissipation $\varepsilon$ the empirical boundary condition (Ekama et al. 1997):

$$
\varepsilon=\frac{k^{3 / 2}}{0.43 H}
$$

is used, where $H$ is the tank depth.

\section{(6) Solid and tank bottom boundaries}

At the rigid walls, the velocities are zero due to the no-slip condition. The universal log-law of the wall (4.68) is implemented in order to take account of the wall shear stress $\tau_{w i}$. For the implementation the boundary layer is divided into two main regions

- in the viscous sublayer $y\left(u_{\tau} / \nu\right) \leq 10$ the wall shear stress is

$$
\tau_{w i}=\rho \bar{u}_{w i}(\nu / y)
$$

- in the turbulent core region $10<y\left(u_{\tau} / \nu\right)<100$ the wall shear stress becomes

$$
\tau_{w i}=\rho \bar{u}_{w i}\left(u_{\tau} \frac{1}{\kappa}\left[\ln \left(\frac{y_{j} u_{\tau}}{\nu}\right)+5.5\right]^{-1}\right),
$$

where $\bar{u}_{w i}$ is the tangential near-wall velocity and $y_{j}$ is its normal distance to the wall.

The friction velocity $u_{\tau}$ can be predicted iteratively from (4.68), that is,

$$
u_{\tau}=\bar{u}_{w i} \frac{1}{\kappa}\left[\ln \left(\frac{y u_{\tau}}{\nu}\right)+5.5\right]^{-1} .
$$

The near-wall values for $k$ and $\varepsilon$ are derived in terms of wall shear stresses using the near-wall assumption that production equals dissipation (see Section 4.6). The near-wall boundary conditions of the turbulent kinetic energy $k$ are

$$
k_{n}=\frac{\left(\tau_{w} / \rho\right)^{2}}{\left(c_{\mu}\right)^{0.5}}
$$


and the dissipation $\varepsilon$ is given by

$$
\varepsilon_{n}=\frac{\left(\tau_{w} / \rho\right)^{3}}{\kappa y_{n}} .
$$

The values for $k_{n}$ and $\varepsilon_{n}$ are applied at the near-wall node as boundary conditions (Hill \& Baskharone 1997).

\subsubsection{Simulating the Darvill old and new SSTs}

The loading condition of Test 3 (De Haas et al. 1998) is chosen; for this case the Darvill old and new tanks are safe. The parameters and loading conditions are listed in Table 6.1 at the end of this chapter. At a $13.3 \%$ higher influent flow the sludge blanket already reaches the effluent outlet with the old tank, while the new tank is still in a stable and safe condition.

Figures 6.14 and 6.15 show the computational results of the Darvill new tank for different meshes. In Figure 6.14 the mesh is of half the size that is used for the actual simulation presented subsequently. As is seen in Figure 6.14, the flow in the inlet region cannot be resolved by using this coarse mesh. Although the algorithm is stable even with a time step of $5 s$, and the computational results show similar results to these of the fine grid, it gives no definite and accurate answer to the density-waterfall in the inlet region.

Figure 6.15 illustrates the simulation of an unstructured mesh, which combines the fine and the coarse mesh by using quadrilaterals and triangles. The fine mesh is used to resolve the flow in the inlet region. A set of triangles are then employed in order to create the transition from the fine to the coarse grid. Although the inlet region is now well-described, the waves resulting from the interaction between potential and kinetic energy are not clearly represented as is the case with the use of a fine mesh (see Figure 6.20).

These calculations demonstrate that the developed algorithm is even stable for coarser meshes. A finer resolution of the mesh is nevertheless needed due to the loss of information by using a large discretization.

For the actual simulation meshes of 85 elements in the horizontal direction and 25 elements in the vertical direction for the old tank, and 58 elements for the new tank in the vertical direction, are chosen, as shown in Figure 6.16. The flow 
pattern of the neutral density case for both tanks is the initial values for the computation of the Darvill SSTs (Figure 6.16).

\section{1) Unsteady and steady flows in the Darvill old tank}

The time-dependent flows in the old tank are illustrated in Figures 6.17 and 6.18. The incoming jet from the inlet chamber moves along the bottom because of its higher density relative to the surrounding fluid. Mass conservation and viscous forces influence the flow pattern in the settling section, resulting in complete flow circulation with a strong bottom forward current and a reverse current at the water surface.

After 15 minutes the sludge current reaches the end of the tank, the region of the upward current to the effluent outlet. The sludge level rises continuously, as does the sludge concentration. The jet coming from the inlet chamber with a smaller density is continuously lifted due to the increasing sludge concentration in the main section of the tank.

The steady state case, illustrated in Figure 6.21 , is reached when the potential and kinetic energies have reached equilibrium. The numerical result shows stable behaviour, as Test 3. The sludge blanket reaches equilibrium and does not rise to the outlet for the clarified water.

Figure 6.21 also illustrates the steady state result with a $13.3 \%$ higher influent flow. Here the sludge blanket reaches the effluent outlet and the current coming from the inlet chamber is raised to the water surface.

Furthermore, Figure 6.21 shows also the distribution of the horizontal velocity $(b, c)$ and the pressure field (d), which is stratified due to the stratification of the suspended solids distribution. In the inlet chamber some kind of checkerboard pressure is seen, and is the result of a too coarse grid in this region.

Additionally, the shifting of the incoming current due to the higher density of the surrounding fluid is clearly seen in Figure $6.21(b, c)$.

\section{2) Unsteady and steady flows in the Darvill new tank}

In Figures 6.19, 6.20 and 6.21 the computational results are shown for the new tank, with influent flow $13.3 \%$ higher than Test 3 (see Table 6.1).

The incoming fluid mixture cascades down, in the process drawing in fluid from 
the inlet chamber. Due to its higher density the inflow has a high potential energy that is converted into kinetic energy at the bottom. No particular construction guides the inflow into the settling section whirling up the hopper region as a result. The entrainment of flow between the influent and the ambient fluid in the tank occurs in the density waterfall, and is clearly seen in Figures 6.19 and 6.20.

The sludge level rises continuously and the sludge current moves along the bottom to the end of the tank, also causing complete flow circulation. The interaction between potential and kinetic energy results in waves that are clearly seen just behind the inlet chamber, firstly due to the impact

The new tank exhibits safe behaviour, which is seen in Figure 6.21. This can be ascribed to the fact that this SST has a much bigger storage zone than the old tank, which leads to stable and constant behaviour over a wide range of loading conditions (see Part 1 of the WRC Report No. 835/1/02, Water Research Commission (2002)).

Table 6.2 gives a summary and comparison with the experimental results, as well as with the computational results predicted with SettlerCAD.

\subsubsection{Simulating the Watts SSTs}

The mesh used has 65 elements in the horizontal direction and 30 elements in the vertical direction, and is shown in Figure 6.22(a).

As mentioned above, the Watts tank is divided into two main sections of similar size: an inlet section of high turbulence, which acts as a mixing and flocculating zone, and the settling section of low turbulence where settling takes place. The use of the $k-\varepsilon$ model is required in order to take into account these regions of differing turbulence. For the modelling of the diffusion coefficient in the concentration equation different modifications have been made, and are described in the next subsection.

Three different loading conditions were chosen: Tests 1 and 4, which showed safe behaviour in the experiments done by Watts et al. (1996), and Test 12, which failed. Ekama \& Marais (2002) show that these three tests were conducted at $56.2,77.5$ and $81.7 \%$ of the idealized 1D flux theory (1DFT) calculated solids loading rate (SLR) limit and the measured capacity of Watts SST was between 
74 and $76 \%$ of the 1DFT SLR limit.

These three test examples illustrate that (i) the simulation results react very sensitively to variation in turbulent viscosity $\nu_{t}$ and diffusivity $\nu_{t} / \sigma_{s}$; (ii) the laminar $\nu_{l} / \sigma_{s}$ and turbulent diffusivity $\nu_{t} / \sigma_{s}$ of the activated sludge is of unknown magnitude and has to be adjusted in order to get the same results as observed in the experiments; and (iii) mass balance cannot always be achieved due to the simplifications inherent in the $2 \mathrm{D}$ model. The different loading conditions are listed in Table 6.3, as well as the model parameters used.

\section{1) Simulating the Watts Test 1}

The simulation of Watts Test 1 showed that an adjustment of some 'free' parameters in the transport and sedimentation sub-model are required. Watts Test 1 is the safest stress test and its loading conditions is given in Table 6.3. Figure 6.23 shows the velocity field and the sludge distribution obtained using a Schmidt number $\sigma_{s}$ of 1 , as well as the predicted turbulent viscosity field. For this case the turbulent diffusivity is equivalent to the predicted turbulent viscosity field.

The simulation predicted failure for Test 1 , which contradicts the observed test results. It prompts the question as to the extent to which the motion of the sludge particles is influenced by diffusion. It is assumed that only the very small sludge particles will be influenced by turbulent diffusion, and they can be related to the poorly settling particles and non-settleable particles.

The flux caused by turbulent diffusion is damped in the higher concentration environment, that is, at the bottom of the tank, due to compaction and hindered movement of the sludge particles. On the other hand the viscosity of the fluid mixture increases with increasing sludge concentration; that is, it cannot be assumed that the eddy diffusivity governing the solid mixing in the tank is proportional to the viscosity of the fluid mixture, especially not in the high concentration range. Inclusion of the buoyancy correction term (4.77) in the equation for the turbulent kinetic energy $k$ leads to unsatisfactory results, in that the turbulence is reduced to zero. Additionally, stability problems result from negative values of the kinetic energy $k$.

Due to the lack of knowledge about the sludge behaviour in turbulent zones and in the high concentration environments, it became necessary to modify the 'turbulent diffusion' parameter. Those simulation results, which showed good 
agreement between the model output of SettlerCAD (as documented in the WRC Report No. 835/1/02, 2002) and the measurements, were used to calibrate the solid distribution. The modification was made in such a way that the diffusion coefficient decreased with increasing concentration, that is, for the Schmidt number $\sigma_{s}$ the local concentration was chosen, as given in Table 6.3.

In Figure 6.24 the steady-state computational result of the Watts tank for stress Test 1 is used to demonstrate the calibration of the Schmidt number $\sigma_{s}$. The Watts tank is safe and the sludge blanket does not reach the outlet of the effluent. The different turbulence in the two sections is clearly seen (Figure 6.24(c)). But this steady state does not yield a mass balance. The required concentration at the first 'suction pipe' cannot be reached. With an average concentration of $4.7 \mathrm{~g} / \ell$ the first suction pipe draws off parts of the incoming flow, which is also clearly seen in Figure 6.24(a,b). This can be ascribed to two facts: firstly, the 2D model cannot simulate a rotating suction lift, with which takes away the fluid discontinuously. In practice the suction lift has a revolution speed of one or two hours, for which the sludge has enough time to accumulate to the required recycle concentration. Secondly, the inflow is too small to achieve sufficient mixing and to create a thickening zone in this region below the inlet.

\section{2) Simulating the Watts Test 12 and Test 4}

The predicted flow pattern and the turbulent viscosity field $\nu_{t}$ of the neutral density case for these examples are shown in Figure $6.22(\mathrm{~b}, \mathrm{c})$. They serve as the initial values for the simulations, and the loading conditions for these stress tests are given in Table 6.3 .

The temporal behaviour before reaching the steady state case is illustrated in Figures 6.25 and 6.26. Already after $100 \mathrm{sec}$ the 'density waterfall', which is clearly seen, causes a circulation opposite to the neutral density case, additionally drawing fluid from the settling section into the inlet section.

The flow pattern and sludge distribution make it clear that the first section acts as a flocculation zone, absorbing kinetic energy caused by the density waterfall and guiding the inlet flow into the second section in which the actual sedimentation takes place.

A stable stratification develops with continuously rising sludge level in the second section. In both sections complete circulations of different intensity arise. 
The steady state case in which potential and kinetic energy have reached equilibrium is shown in Figure 6.27. Stress test 12 causes failure of the Watts tank. The sludge blanket reaches the effluent level. The height of the thickening zone allows only a small gap below the skirt baffles for the inflow to reach the settling section. The incoming jet is raised due to the higher sludge concentration in the settling section.

The computational result of Test 4 is shown in Figure 6.27. With $97.5 \%$ this steady state does not yield a mass balance, but the sludge blanket reaches equilibrium and does not rise to the effluent outlet.

The results are listed in Table 6.4 and compared with the experimental results, as well as with the computational results predicted with SettlerCAD, documented in Part I of the WRC Report No. 835/1/02 (2002).

\subsection{Conclusion}

- The algorithm and modelling techniques developed in the preceding chapters and implemented in a finite element program have been successfully applied to the modelling of secondary settling tanks.

- It has been shown that the developed finite element code is stable for coarse meshes even for meshes that cannot resolve the density-driven flow accurately.

- A grid test simulation showed how easy it is to refine the mesh and to construct an unstructured mesh by using different elements, such as the combination of quadrilaterals and triangles.

- The Darvill old and new tank has been modelled with a constant turbulent viscosity and diffusivity, respectively. In order to achieve the results obtained from measurements the Schmidt number had to be adjusted to a value larger than one, which is in contrast to the recommended values found in the literature.

- It has been possible to show qualitatively the flow pattern and sludge distribution of these two SSTs, which in turn give a better understanding with regard to their different behaviour in general, and to the superiority and better efficiency of the new tank to the old tank, in particular. 
- The special construction of the Watts SST divides the Watts tank into two main sections of similar size: an inlet section of high turbulence, which acts as a mixing and flocculating zone, and the main section of low turbulence, where settling takes place. To take the different turbulence regions and their interaction into account the use of the $k-\varepsilon$ model was made for the simulation of the Watts tank.

- The simulation of Watts Test 1, which is the safest stress test, predicted failure and therefore contradicts the observed test results. As the laminar $\nu_{l} / \sigma_{s}$ and turbulent diffusivity $\nu_{t} / \sigma_{s}$ of the activated sludge is an unknown size, it has to be adjusted in order to get the same results as observed in the experiments. For the modelling of the diffusion coefficient in the concentration equation different modifications have been made. Best results could be reached by using Schmidt numbers $\sigma_{s}$ larger than 1 .

- It was observed that mass balance cannot always be achieved. This can either be ascribed to the necessary simplifications of $2 \mathrm{D}$ models, for example a rotating suction lift, which takes away the fluid discontinuously, cannot be modelled and the required recycle sludge concentration cannot be attained. Furthermore the described mathematical model does not consider sludge compaction due to the hydrostatic pressure on the bottom of the tank. Those stress tests with very high recycle sludge concentration will fail, because sludge compaction is not taken into account.

- In general, flow and sludge distribution react very sensitively to the turbulent viscosity and diffusivity. The assumption that the eddy diffusivity, which governs the solids mixing in the tank, is proportional to the eddy viscosity, cannot be confirmed, and the sludge is less influenced by diffusion than is assumed by using the Reynolds analogy. 

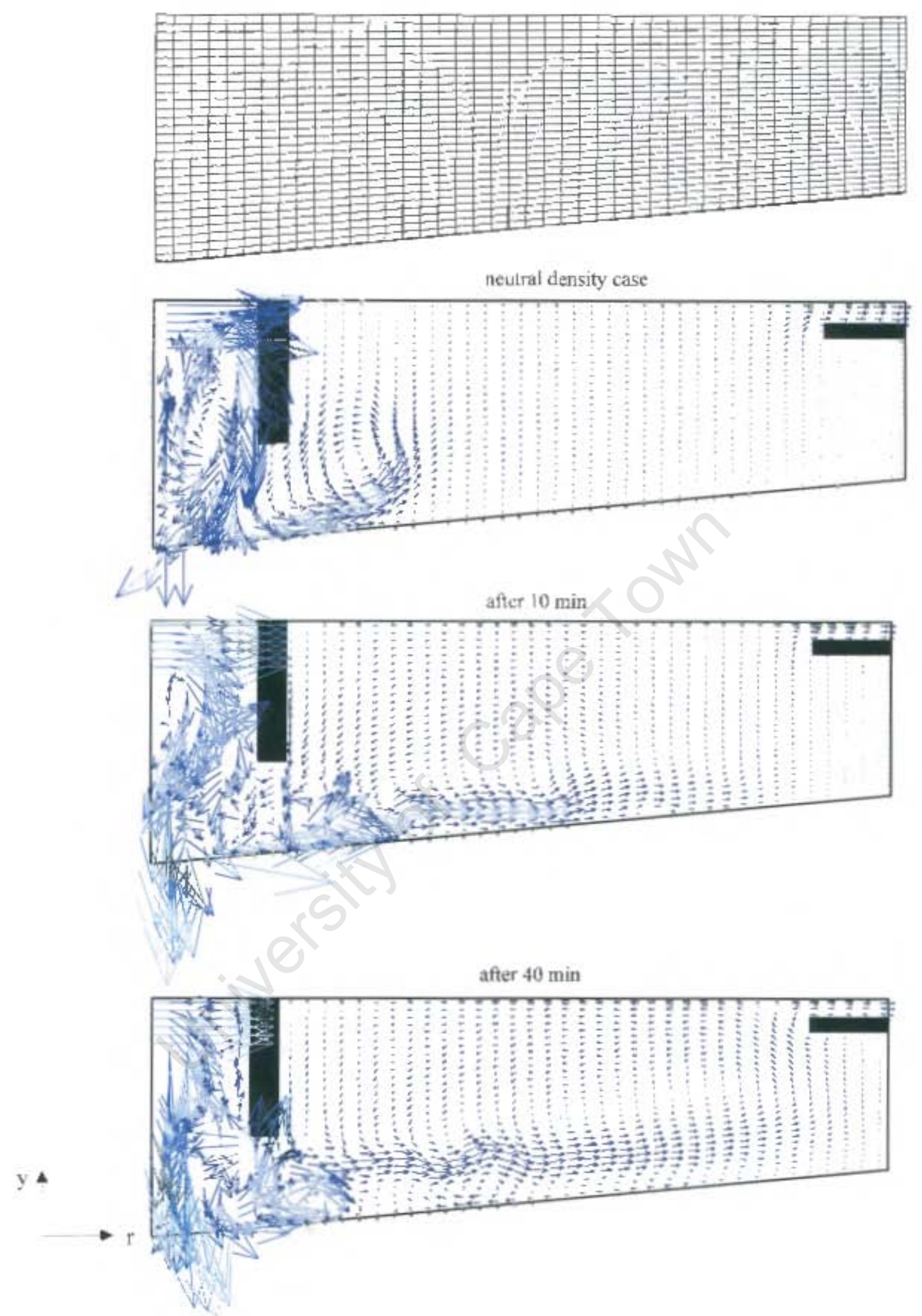

Figure 6.14: Coarse mesh of Darvill new tank (43 x 29), and the flow pattern for the neutral density case, as well as for the solids loaded flow after 10 and 40 min. 

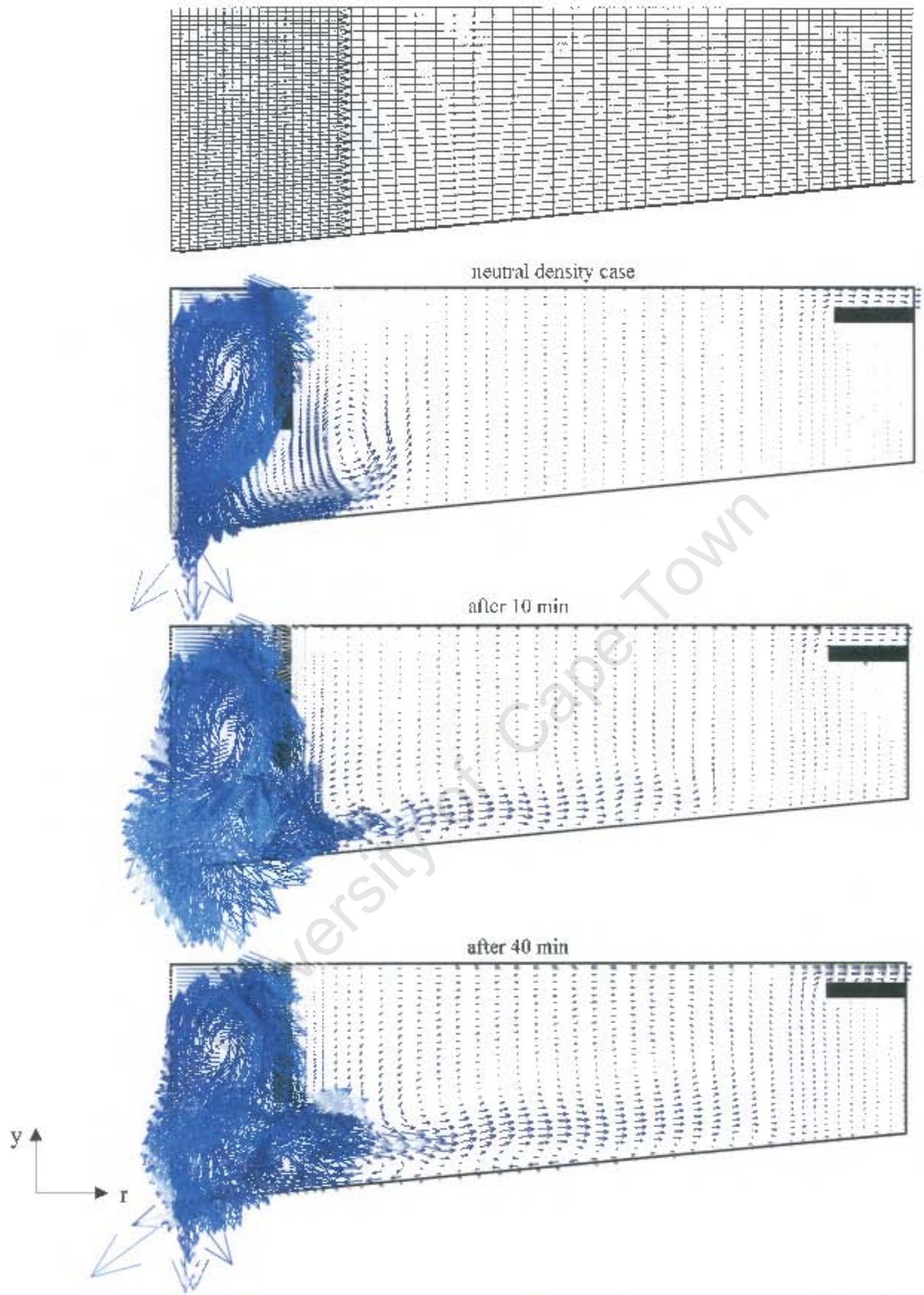

Figure 6.15: Unstructured mesh of Darvill new tank (20 $\times 58+34 \times 29)$, and the flow pattern for the neutral density case, as well as for the solids loaded flow after 10 and $40 \mathrm{~min}$. 
(a)

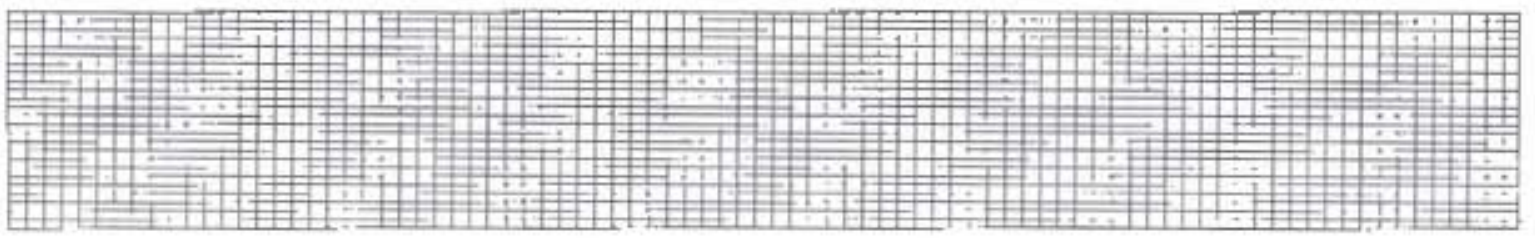

(b)

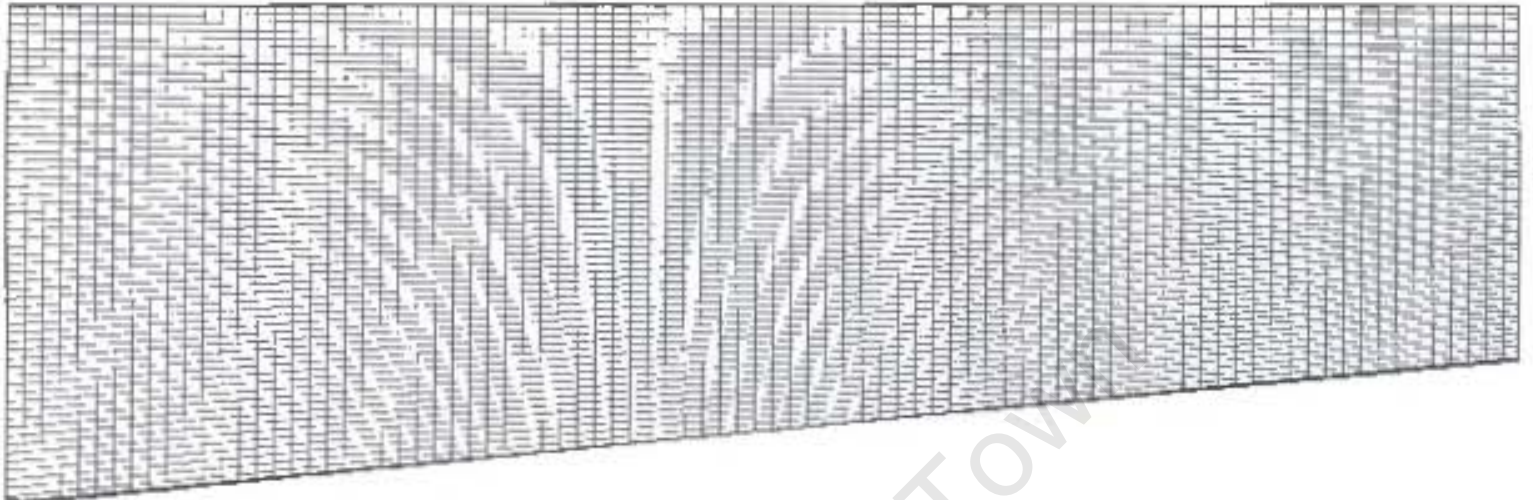

(c)
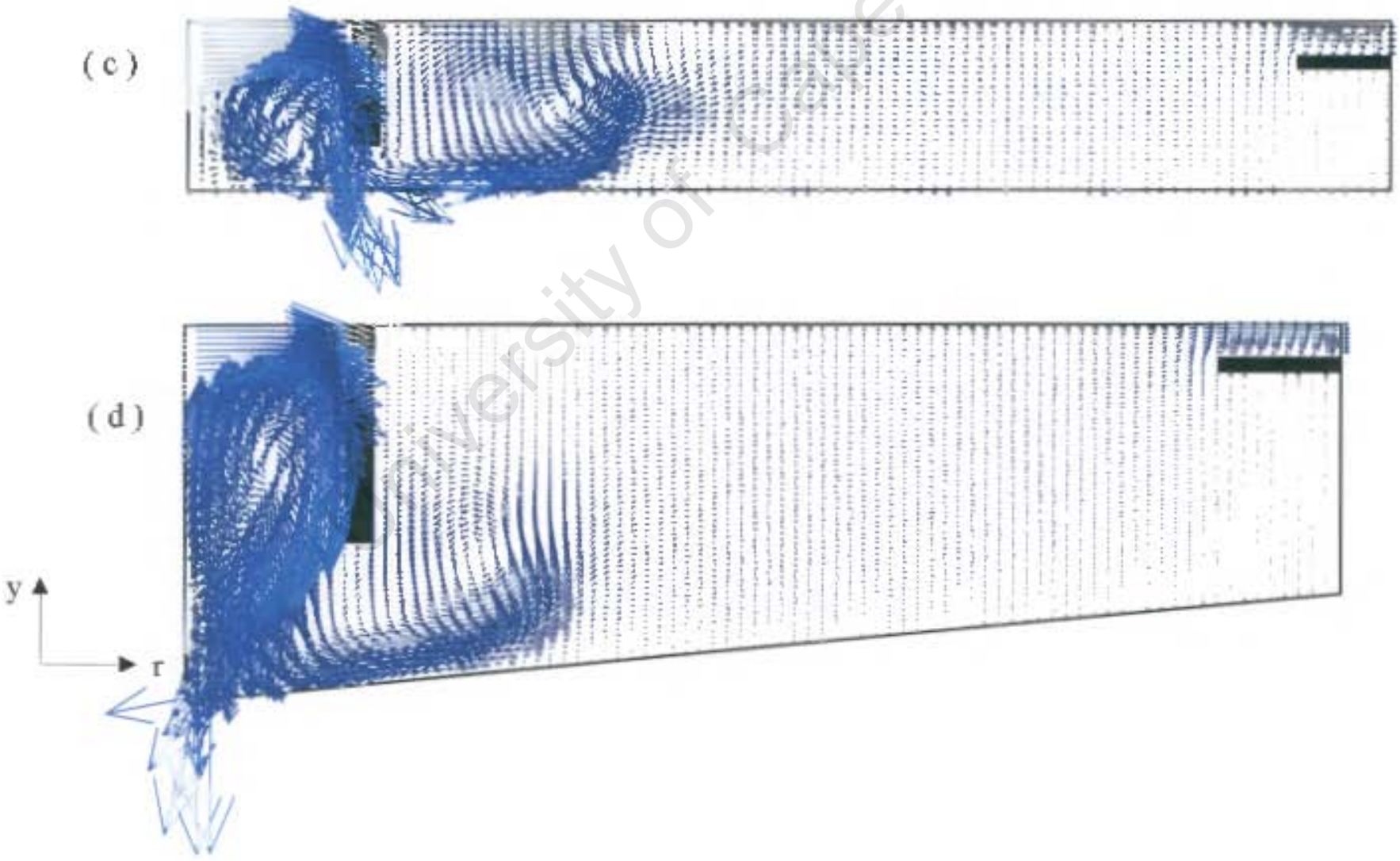

Figure 6.16: Mesh of $85 \times 25$ elcments for Darvill old tank (a) and of $85 \times 58$ elements for Darvill new tank (b), as well as the flow patterns for the neutral density case ( $c$ and $\mathrm{d}$ ). 
$100 \mathrm{sec}$
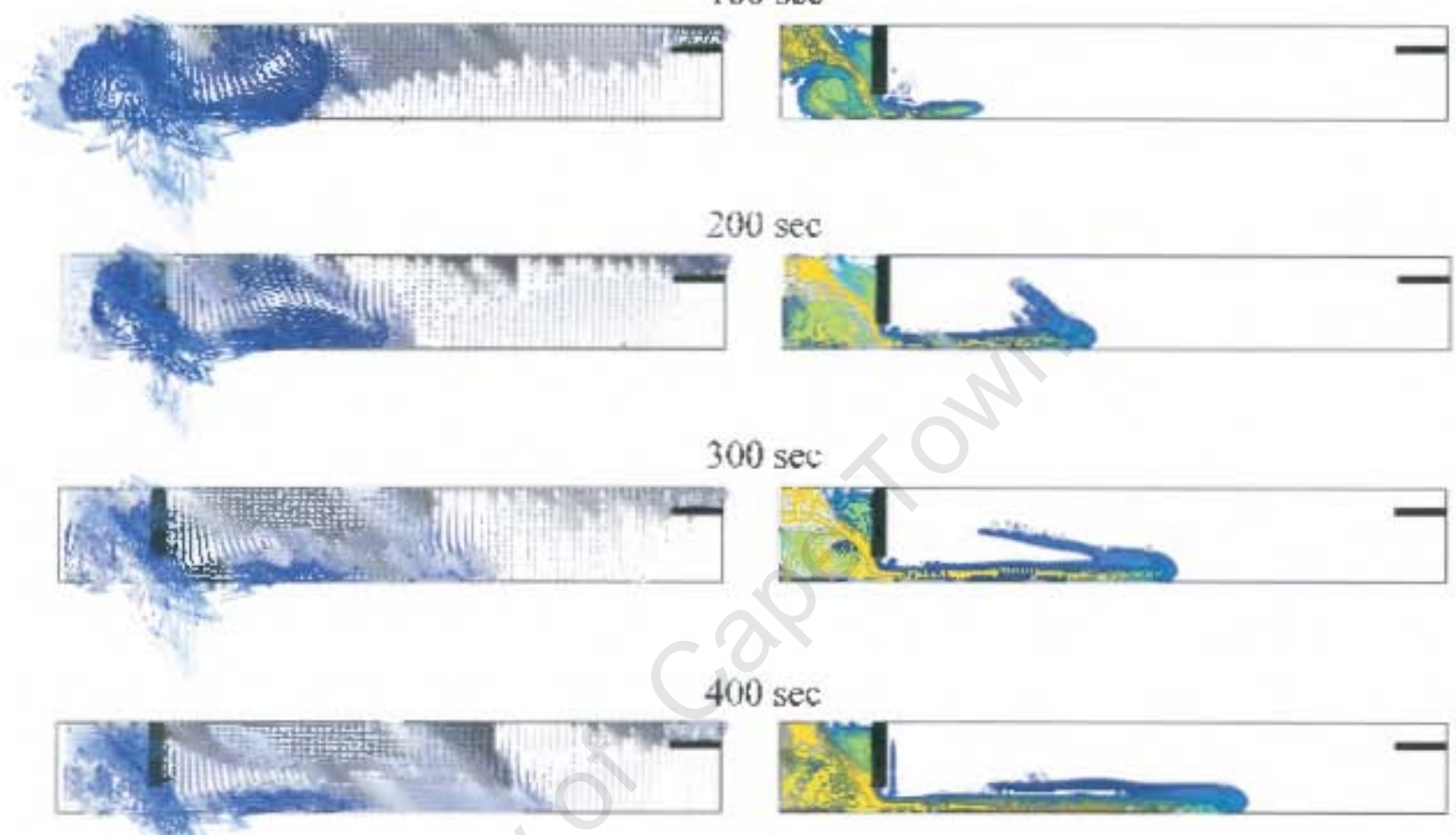

$500 \mathrm{sec}$

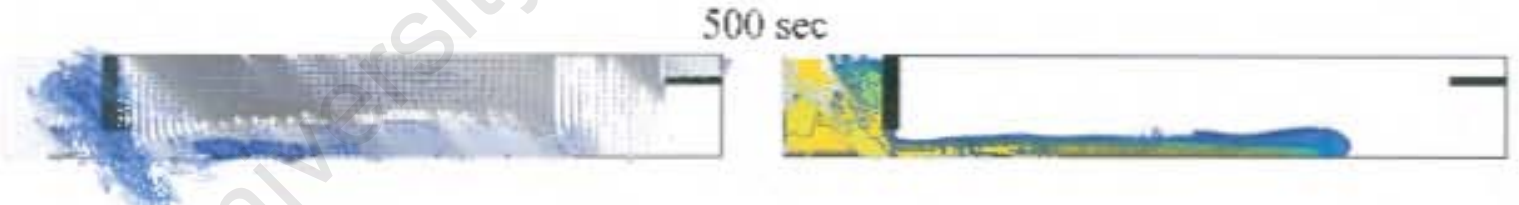

$10 \min$
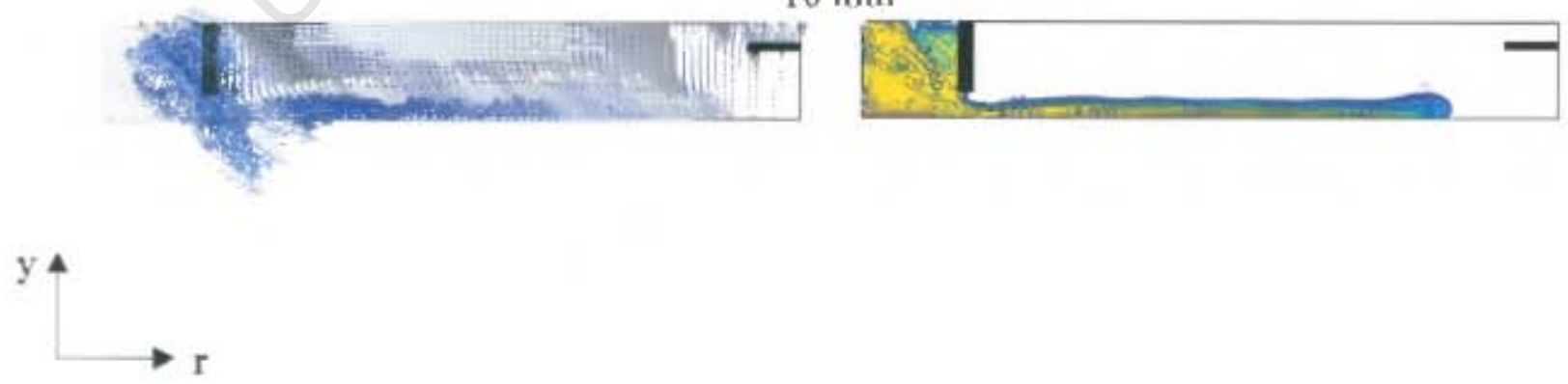

Figure 6.17: Darvill old tank: After $100 \mathrm{sec}$ to after $10 \mathrm{~min}$, predicted flow pattern and sludge distribution $c$ in $g / \ell$. 
15 min

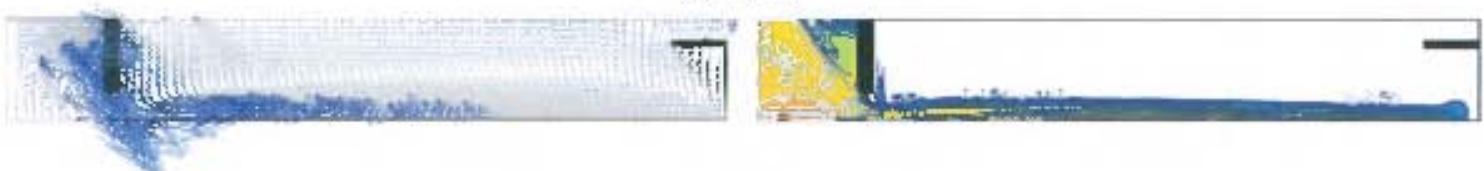

$20 \mathrm{~min}$

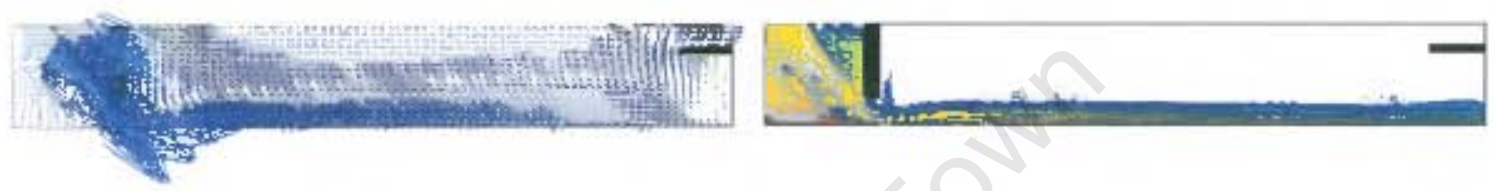

30 min

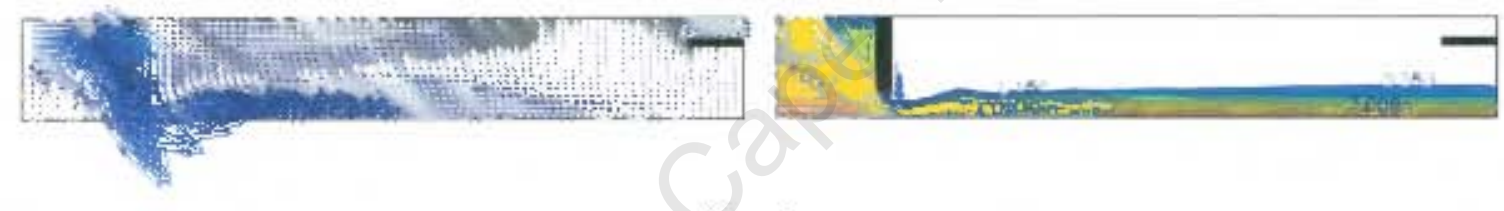

$60 \mathrm{~min}$

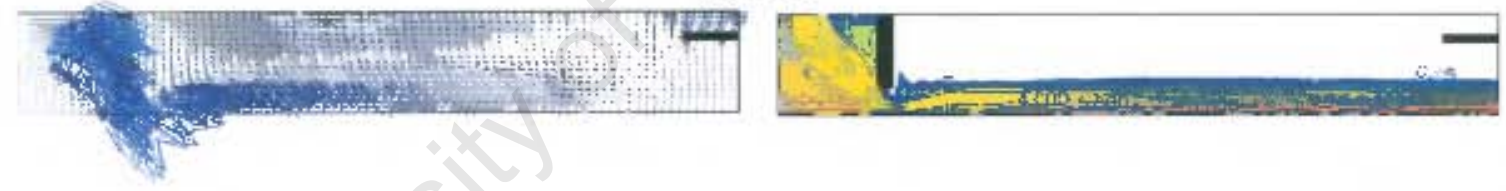

6 hours
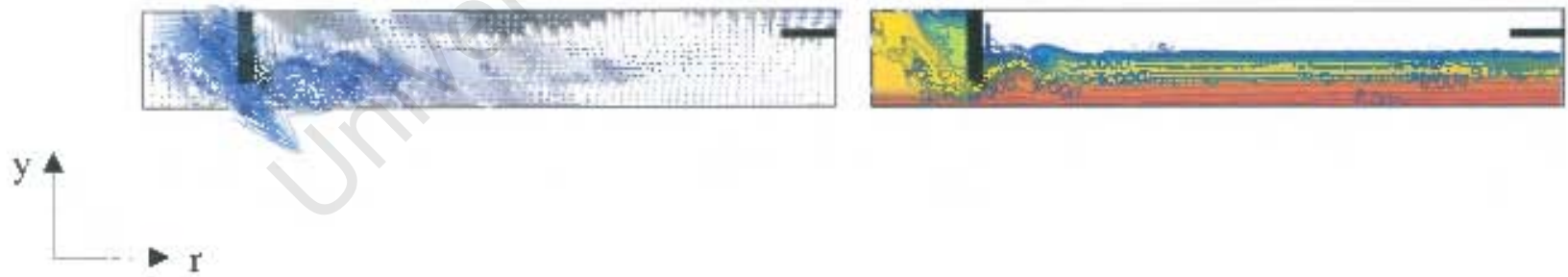

Figure 6.18: Darvill old tank: After 15 min to after 6 bours, prestictod flow pattern and sludge distribution $c$ in $g / \ell$. 
$100 \mathrm{sec}$

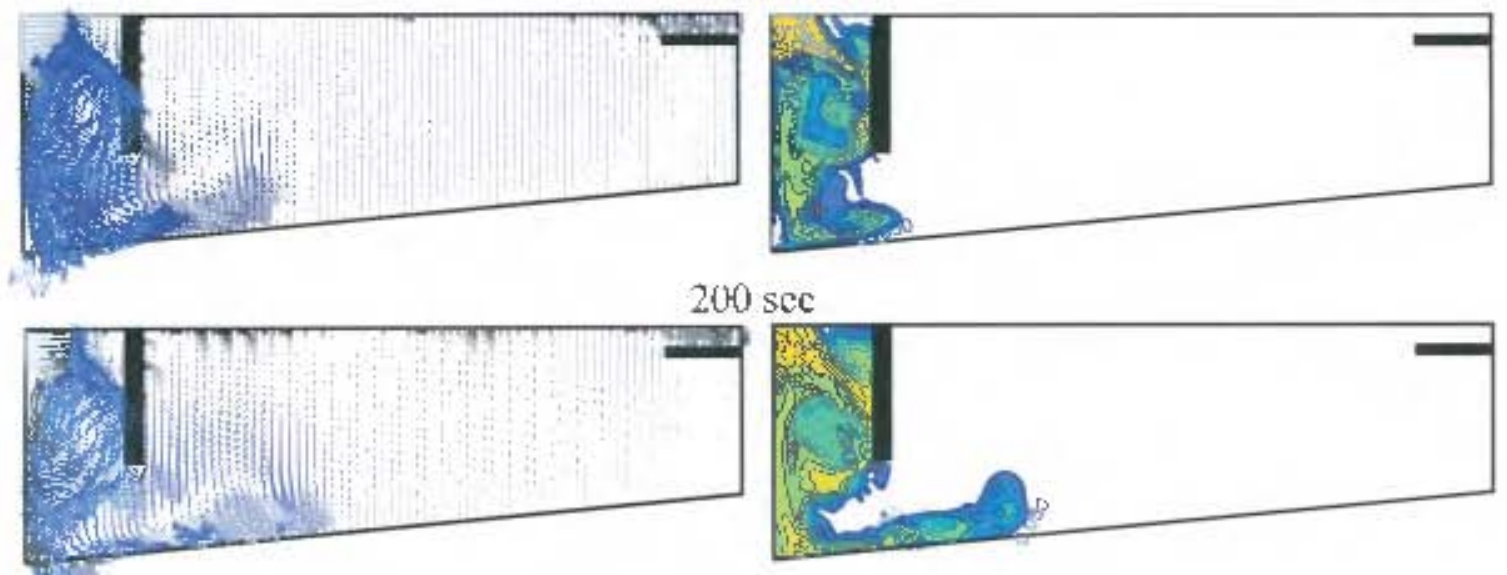

$300 \mathrm{sec}$

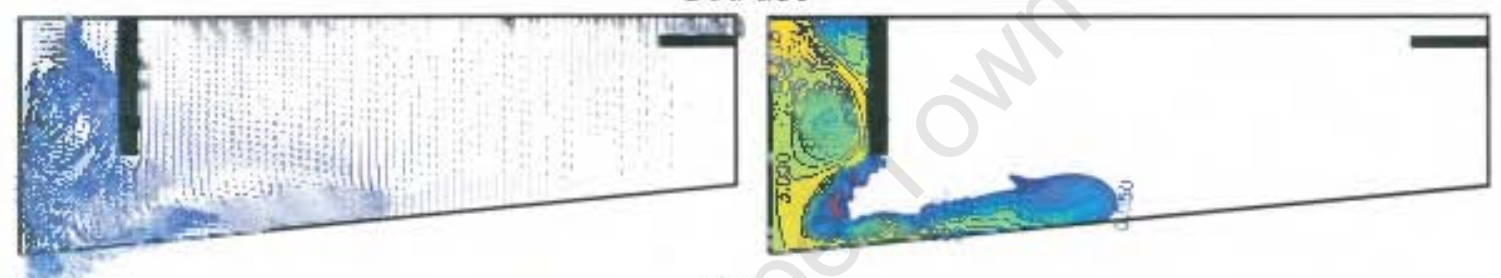

$400 \mathrm{sec}$

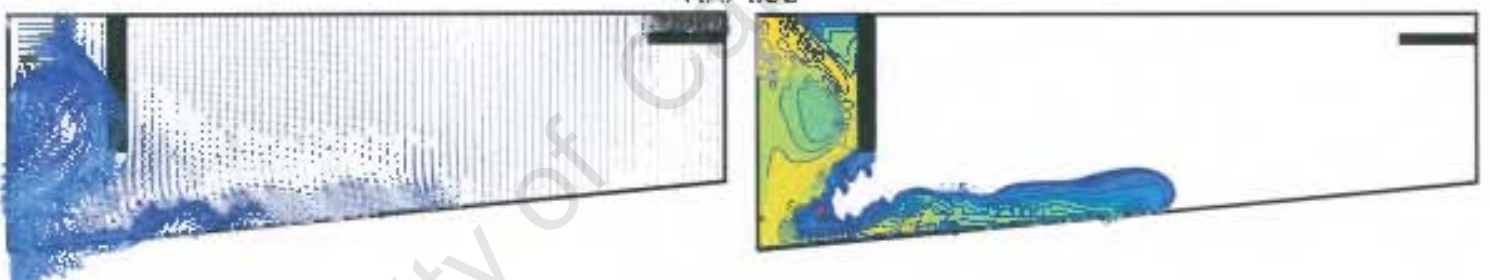

$500 \mathrm{sec}$

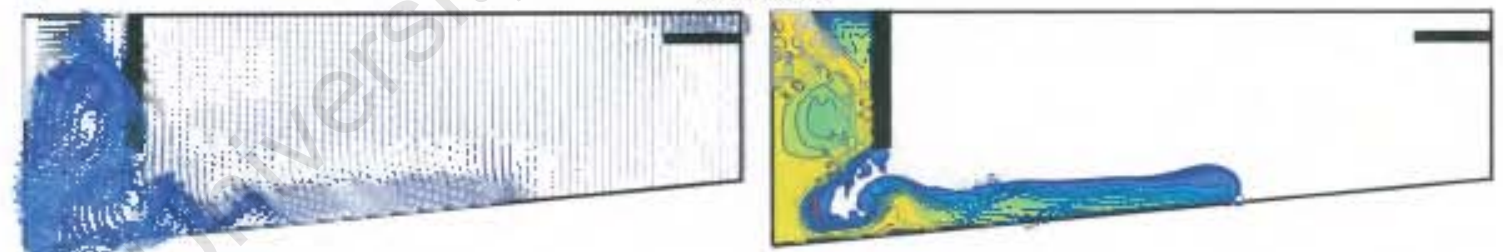

$10 \mathrm{~min}$
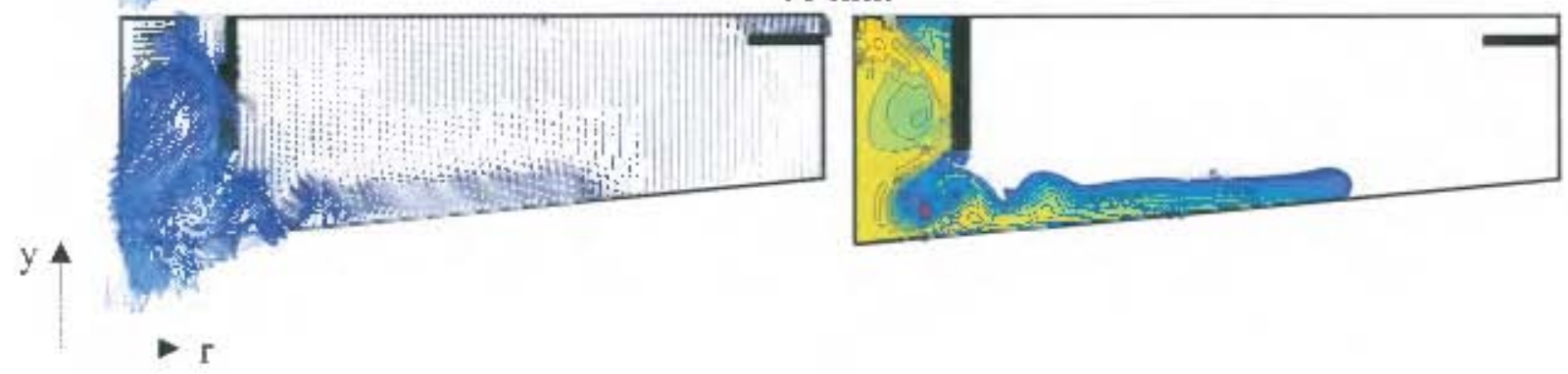

Figure 6.19: Darvill new tank with a $13.3 \%$ higher influent flow: After 100 sex: to after $10 \mathrm{~min}$, predicted llow pattern and sludge distribution $c$ in $g / \ell$. 
$15 \mathrm{~min}$
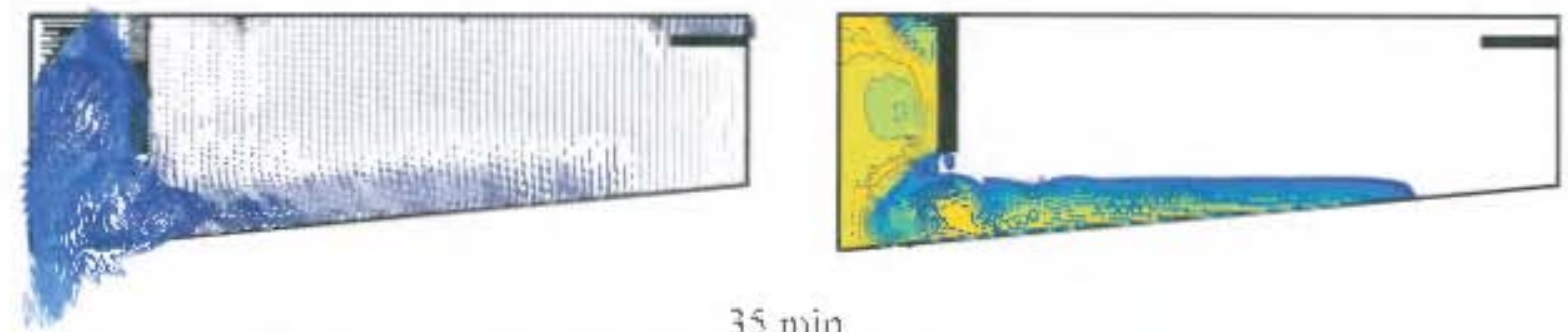

35 ग)
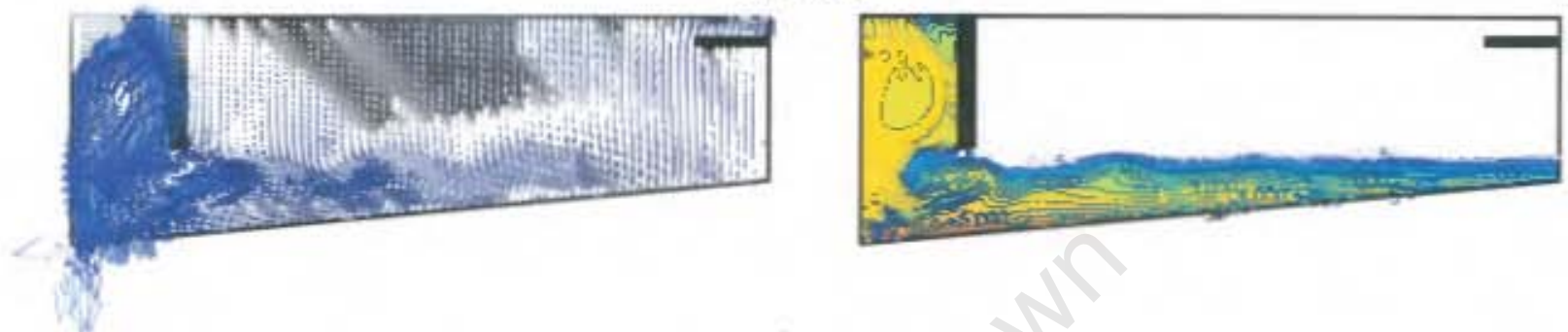

40 min
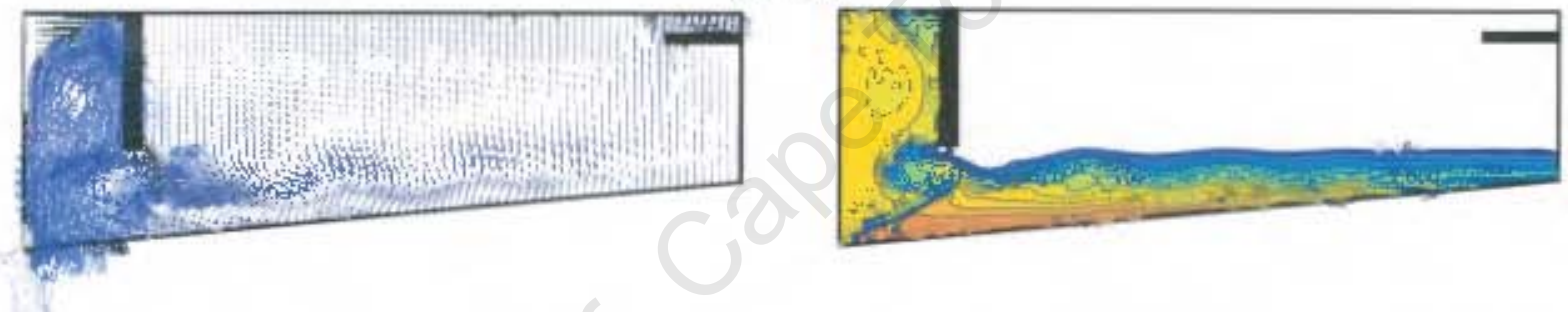

$60 \mathrm{~min}$
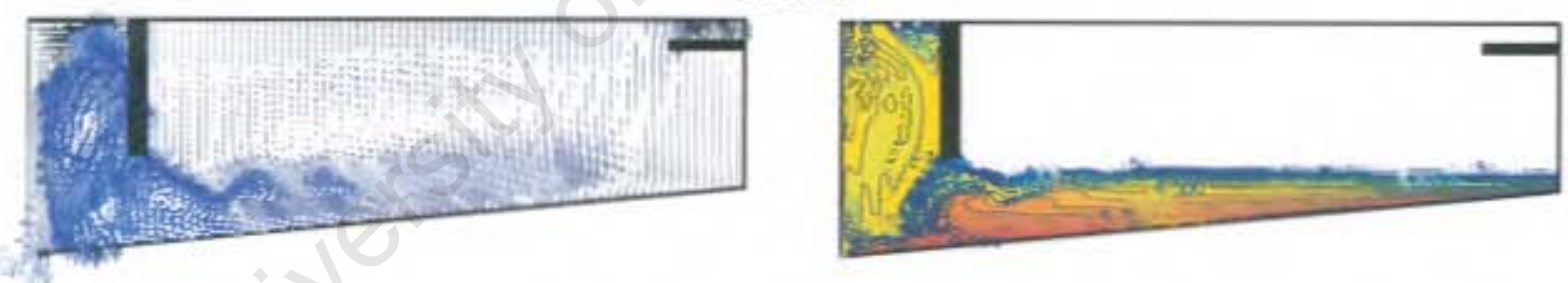

6 hours
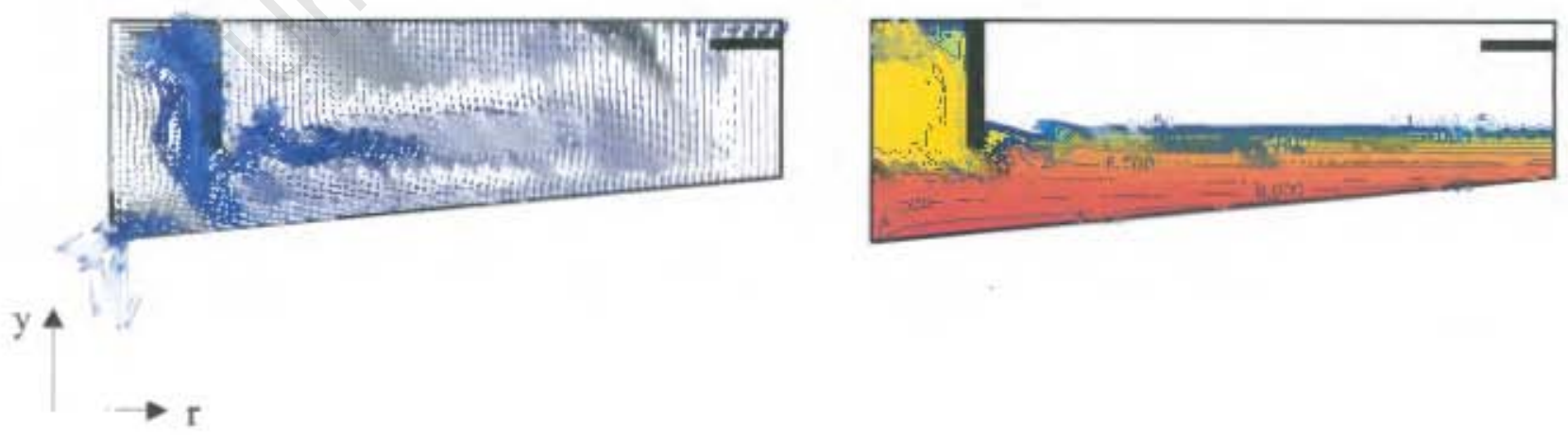

Figure 6.20: Darvill ncw tank with a $13.3 \%$ higher influent flow: After 10 min to after 6 hours, prodicted flow pattern and sludge distribution $c$ in $g / \ell$. 
Darvili old lank

(a)

(b)

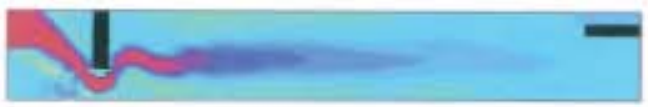

(c)

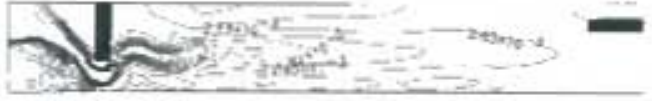

(d)

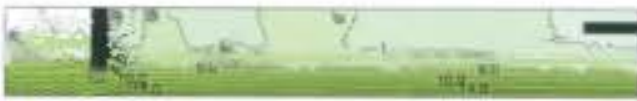

(e)

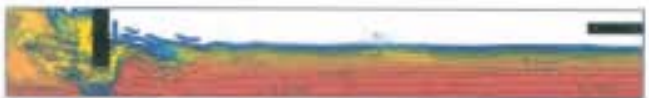

Darvill old tank - $13.3 \%$
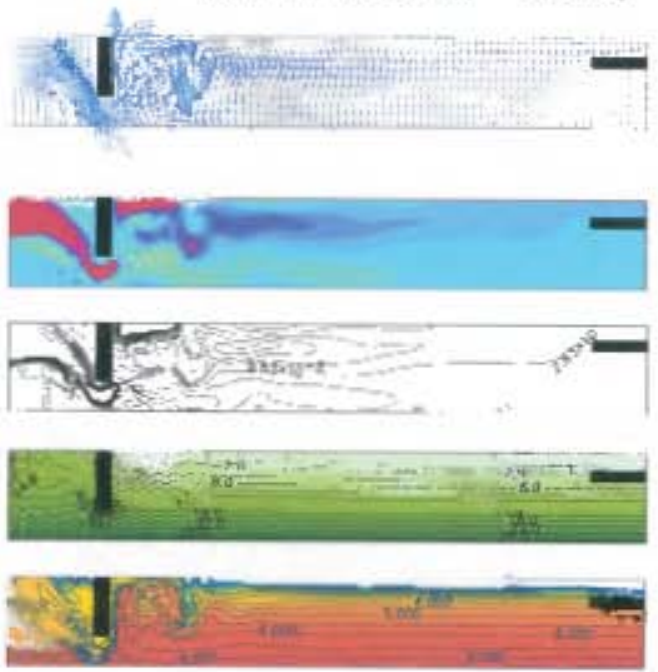

[)arvill now tank $+13.3 \%$

(a)

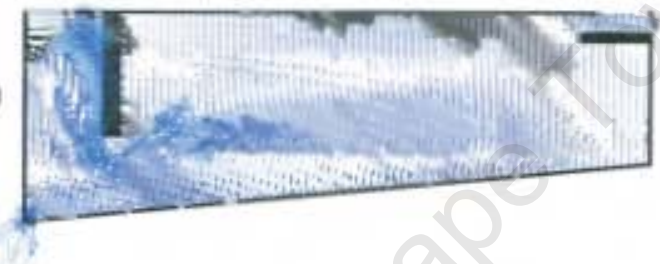

(b)

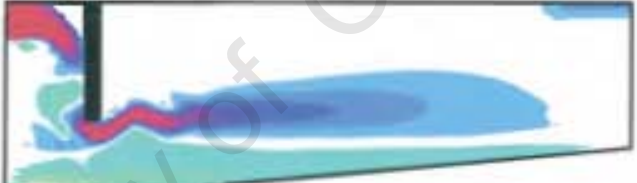

(c)

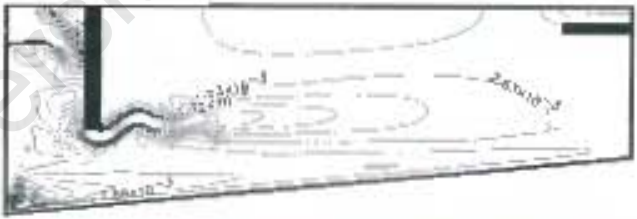

(d)
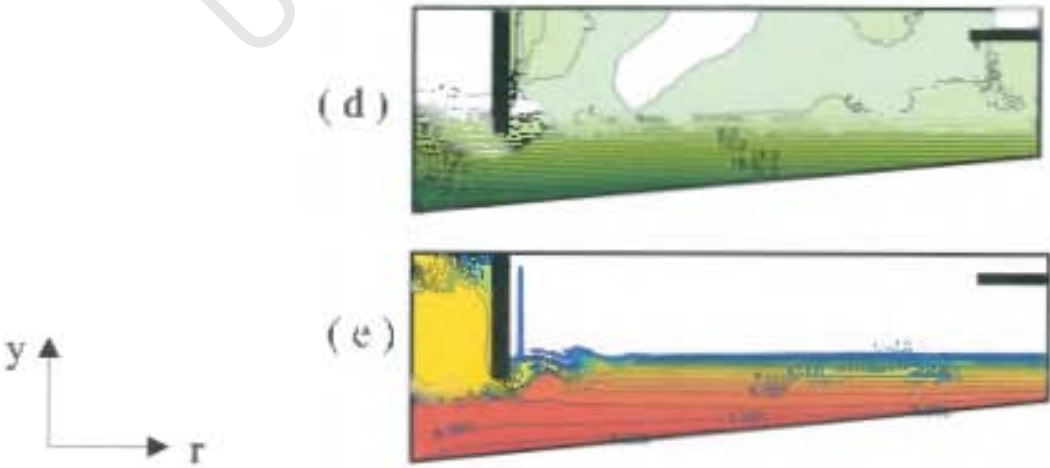

Figure 6.21: Darvill old and new tanks: Steady state case, predicted flow pattern (a), horizontal velocity $u$ in $m / s$ (b,c), pressure field in $P a$ (d) and sludge distribution $c$ in $g / \ell(\mathrm{e})$. 
(a)

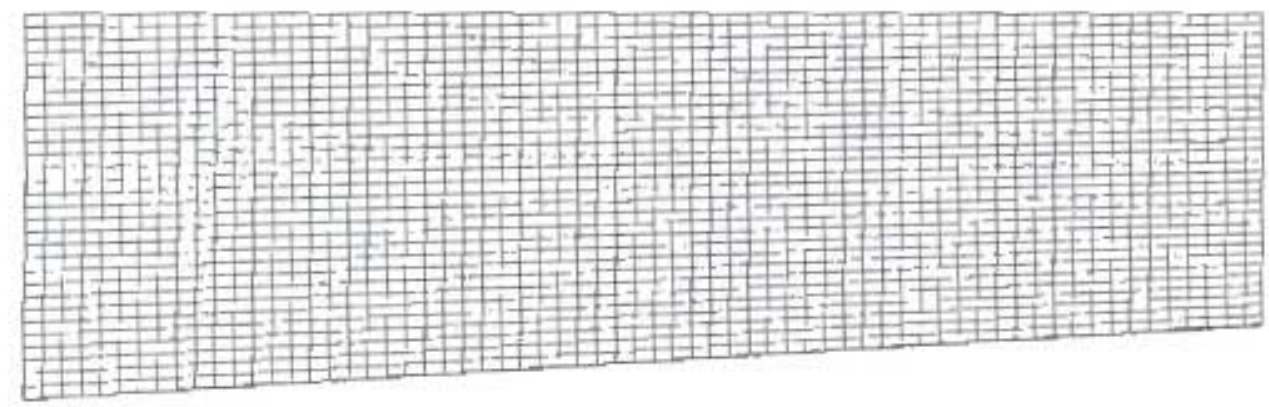

(b)

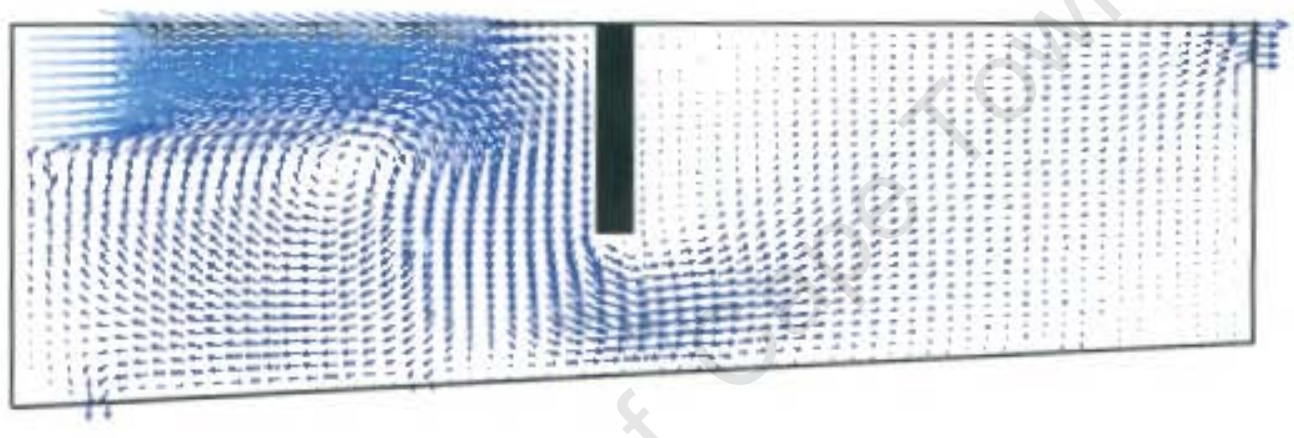

(c)

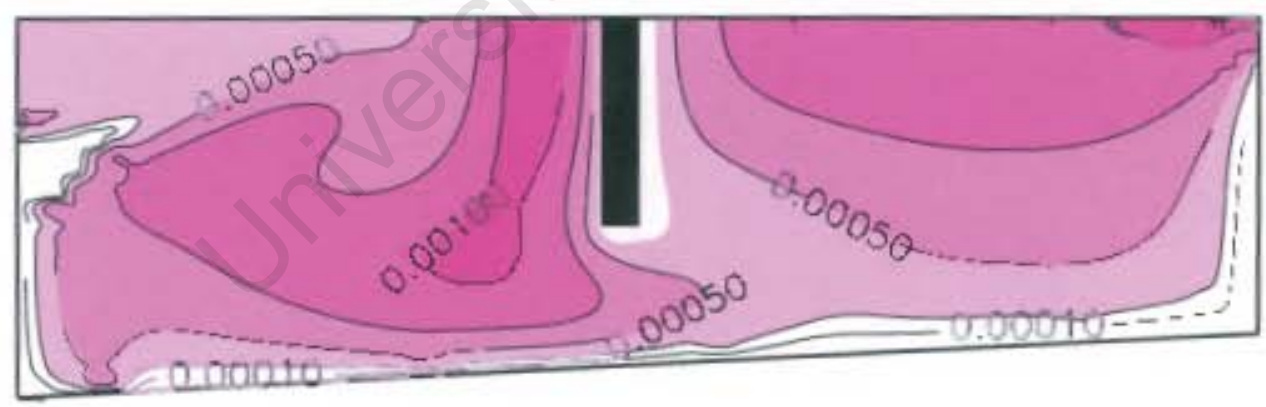

y

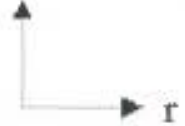

Figure 6.22: Watts tank: Mesh of $65 \times 30$ elements (a), predicted flow pattern for the neutral density case (b), predicted turbulent viscosity $\nu_{t}$ field for the neutral density case in $m^{2} / s$ (c). 
(a)

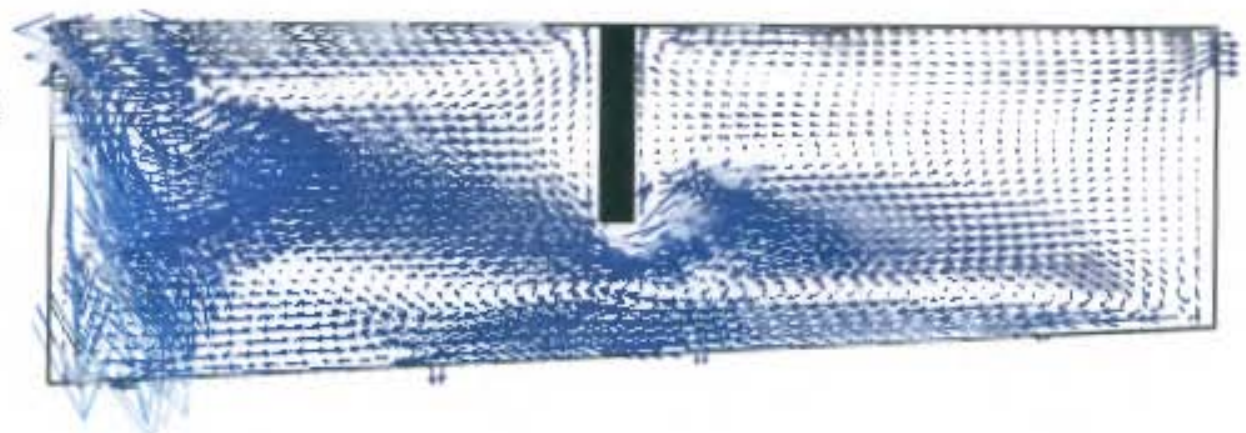

(b)

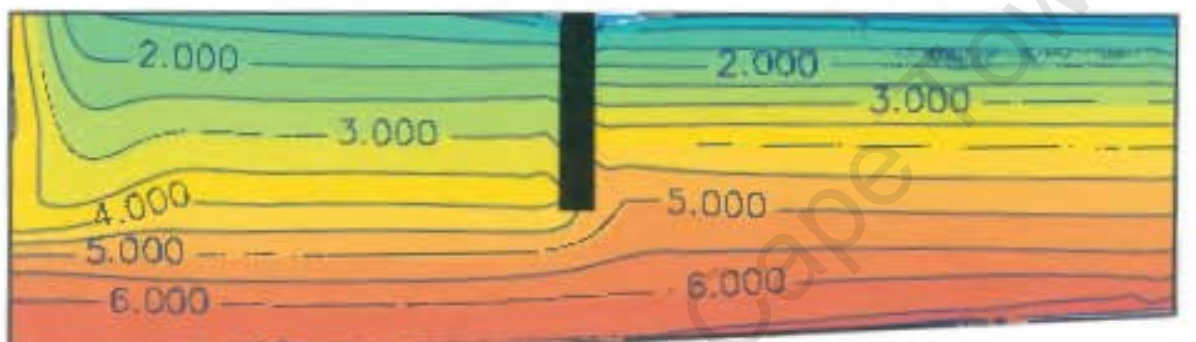

(c)
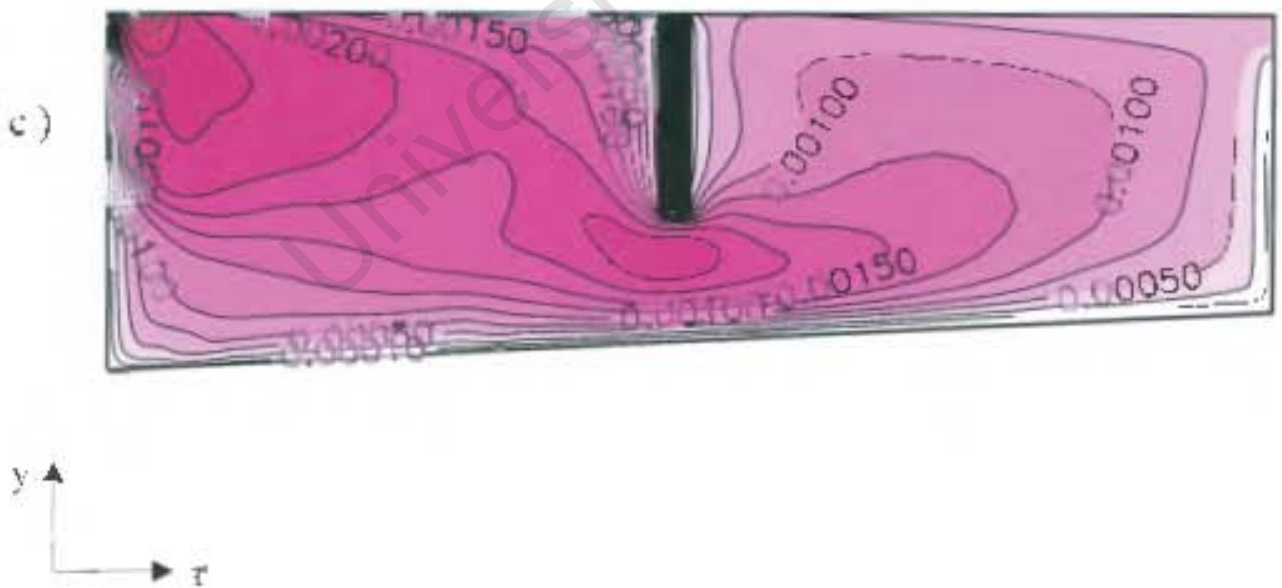

Figure 6.23: Watts test 1 with a Schmidt number of 1: predicted flow pattern (a), sludge distribution $c$ in $g / \ell$ (b) and predicted turbulent viscosity $\nu_{t}$ field in $m^{2} / s(c)$. 


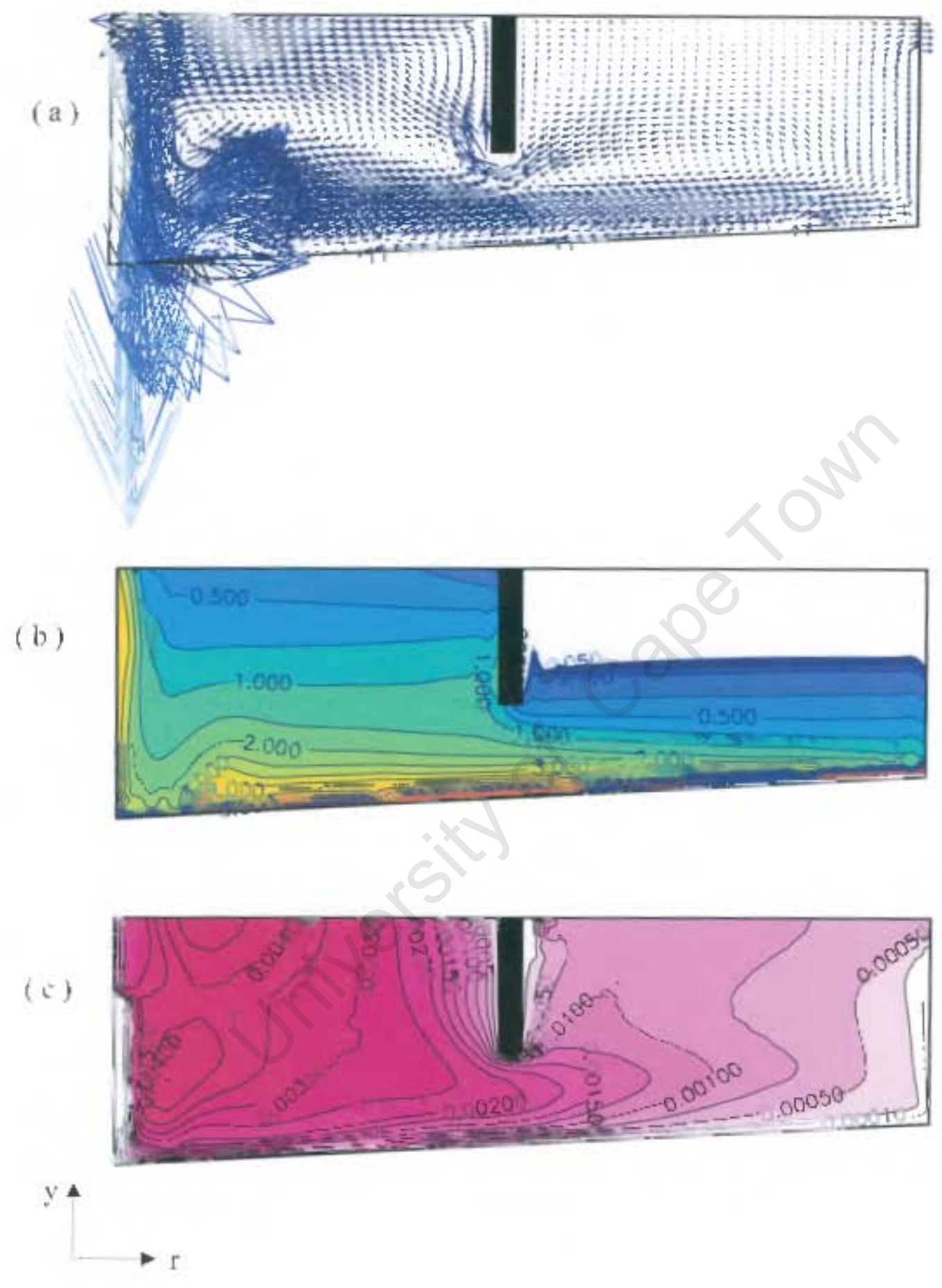

Figure 6.24: Watts test 1 with a Schridt number of 5: predicted flow pattern (a), sludge distribution $c$ in $g / \ell$ (b) and predicted turbulent viscosity $\nu_{l}$ field in $\mathrm{m}^{2} / \mathrm{s}(\mathrm{c})$. 
$100 \mathrm{sec}$

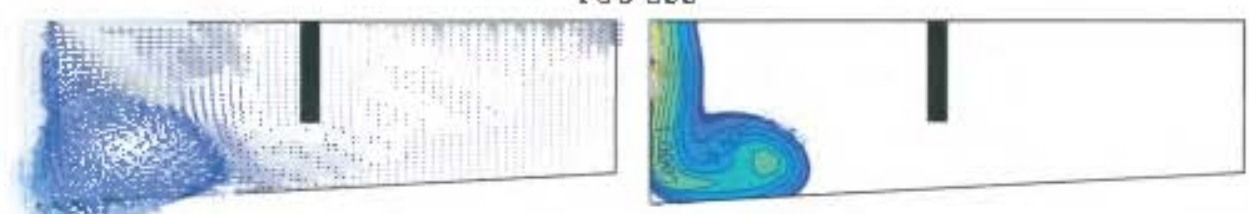

$200 \mathrm{sec}$

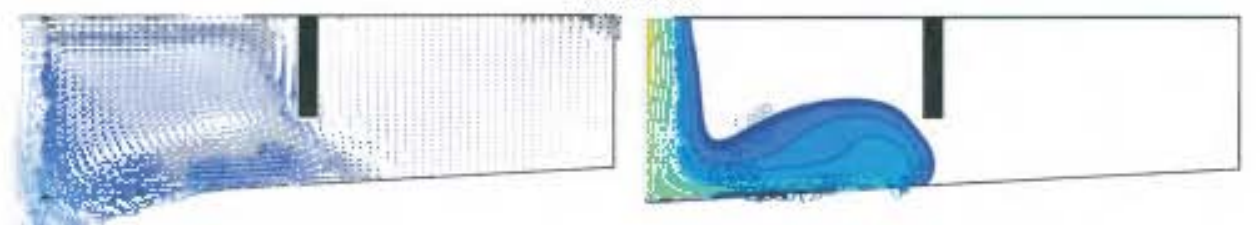

300 sec

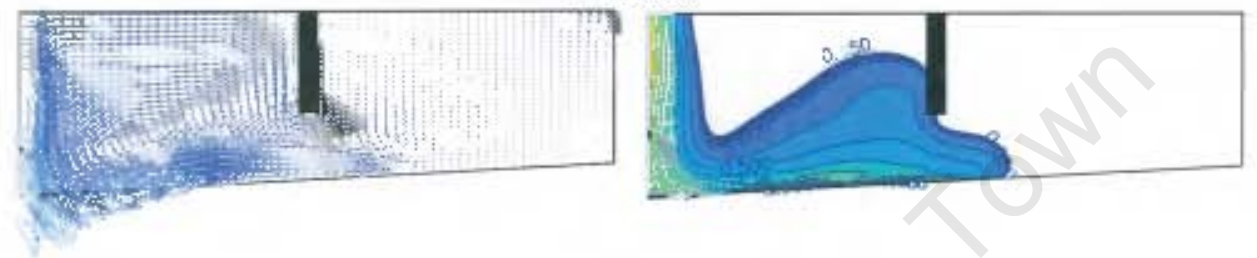

$400 \mathrm{sec}$

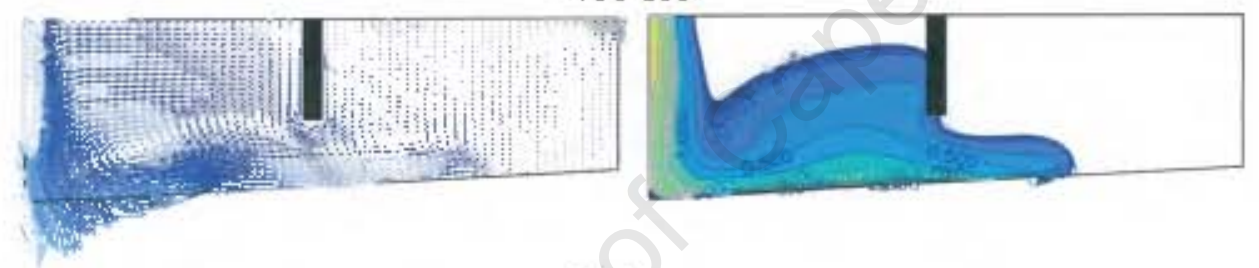

$500 \mathrm{sec}$
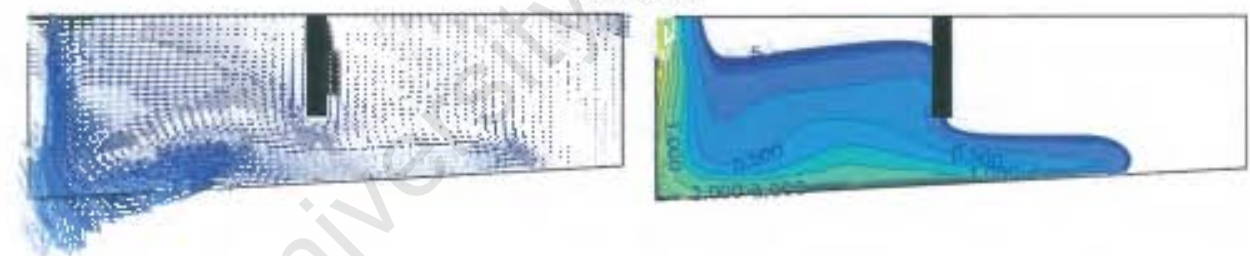

$10 \mathrm{~min}$
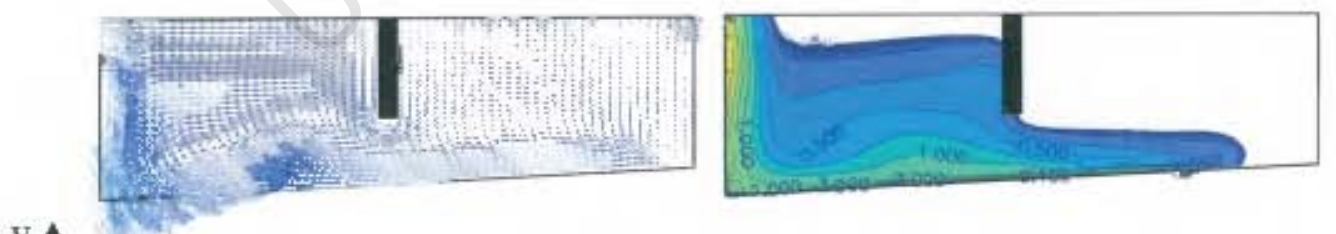

y 4

Figure 6.25: Watts test 12: After $100 \mathrm{sec}$ to after 10 min, predicted flow pattern and sludge distribution $c$ in $g / \ell$. 
15 min
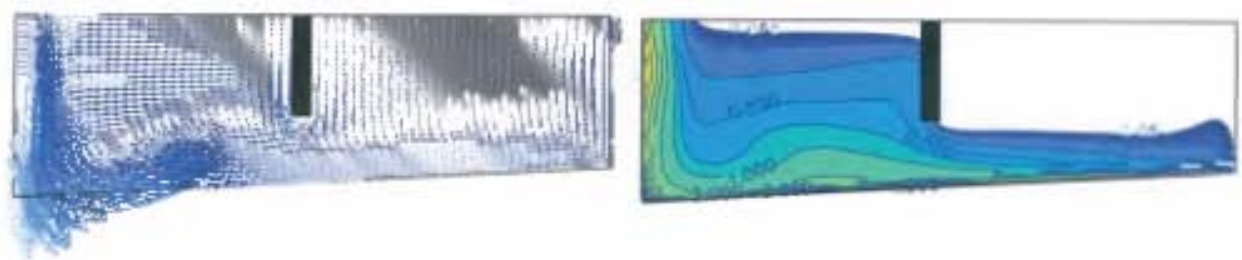

$20 \mathrm{~min}$

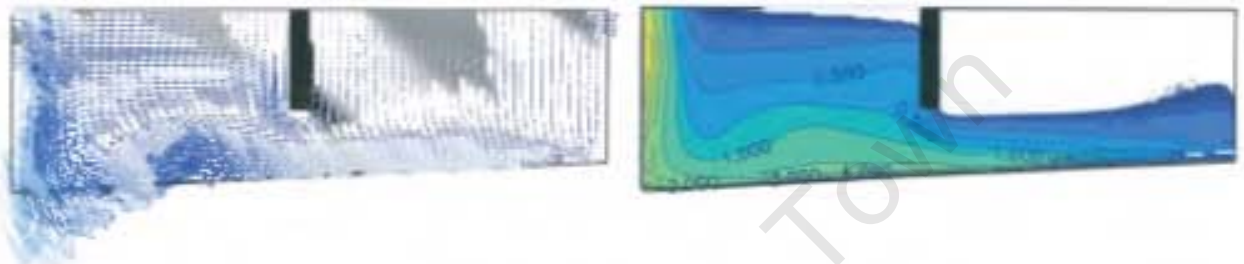

$30 \mathrm{~min}$
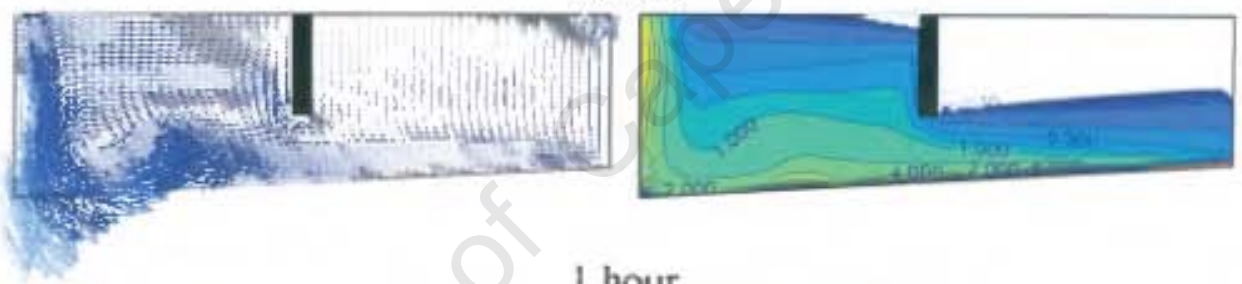

1 hour

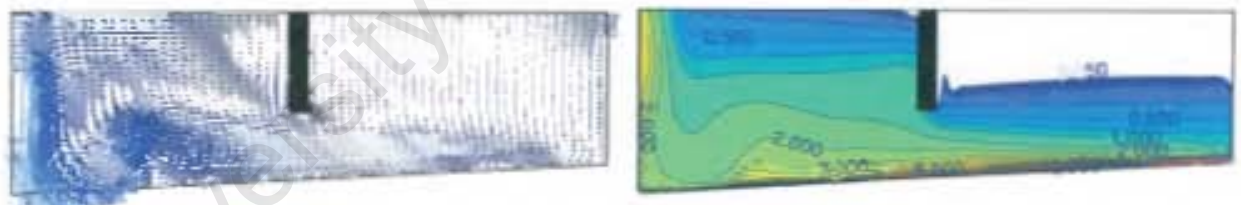

6 hours
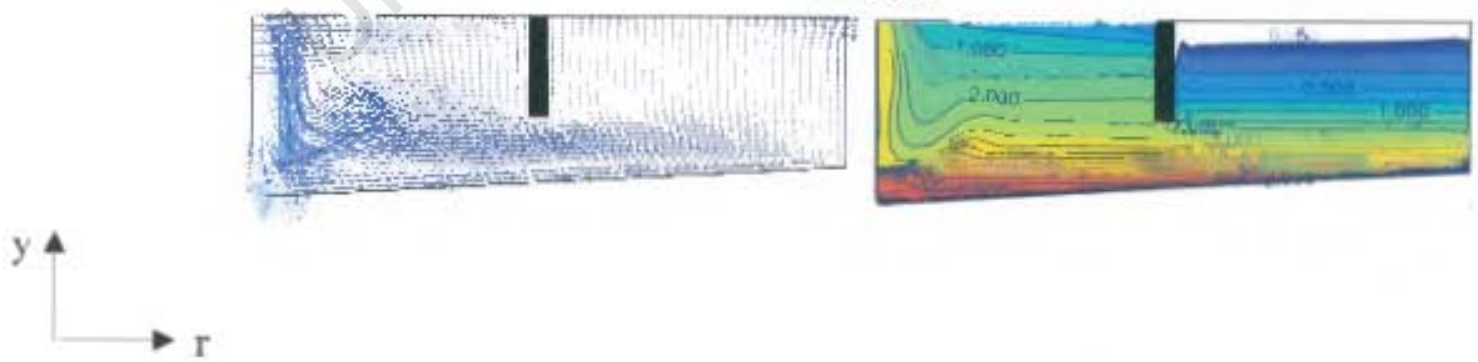

Figure 6.26: Watts test 12: After $10 \mathrm{~min}$ to after 6 hours, predicted flow pattern and sludge distribution $c$ in $g / \ell$. 
Watts Test 12

(a)

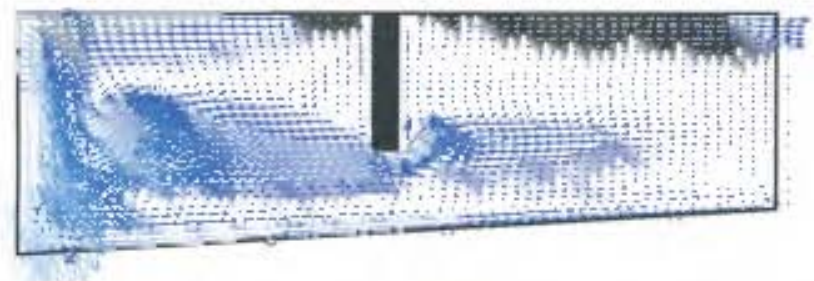

(b)

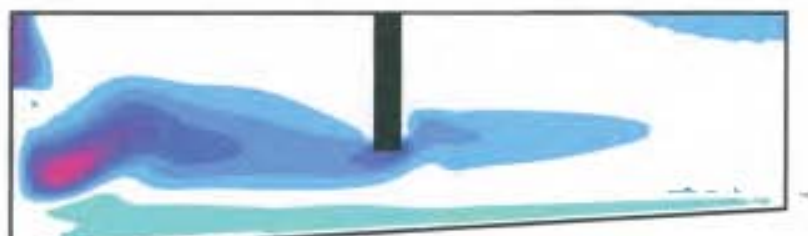

(c)

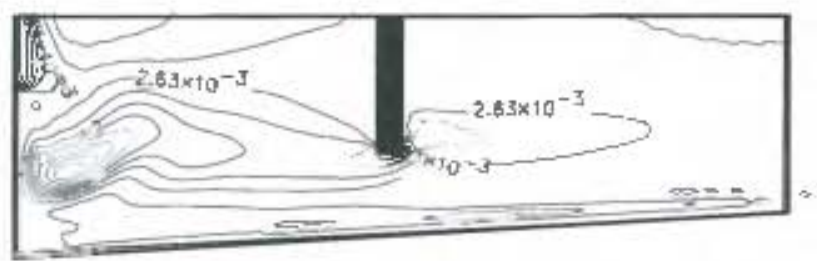

(d)

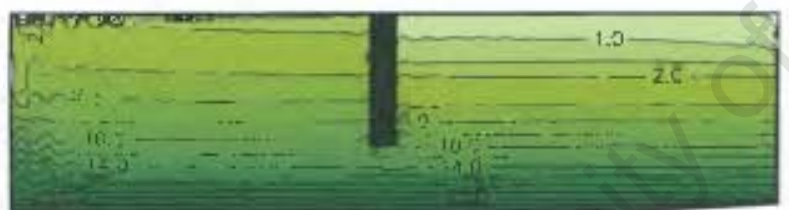

(c)

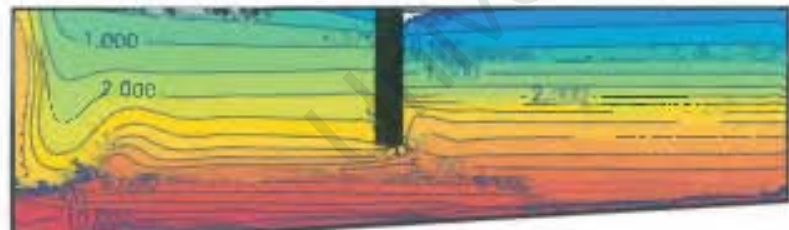

Watts Test 4
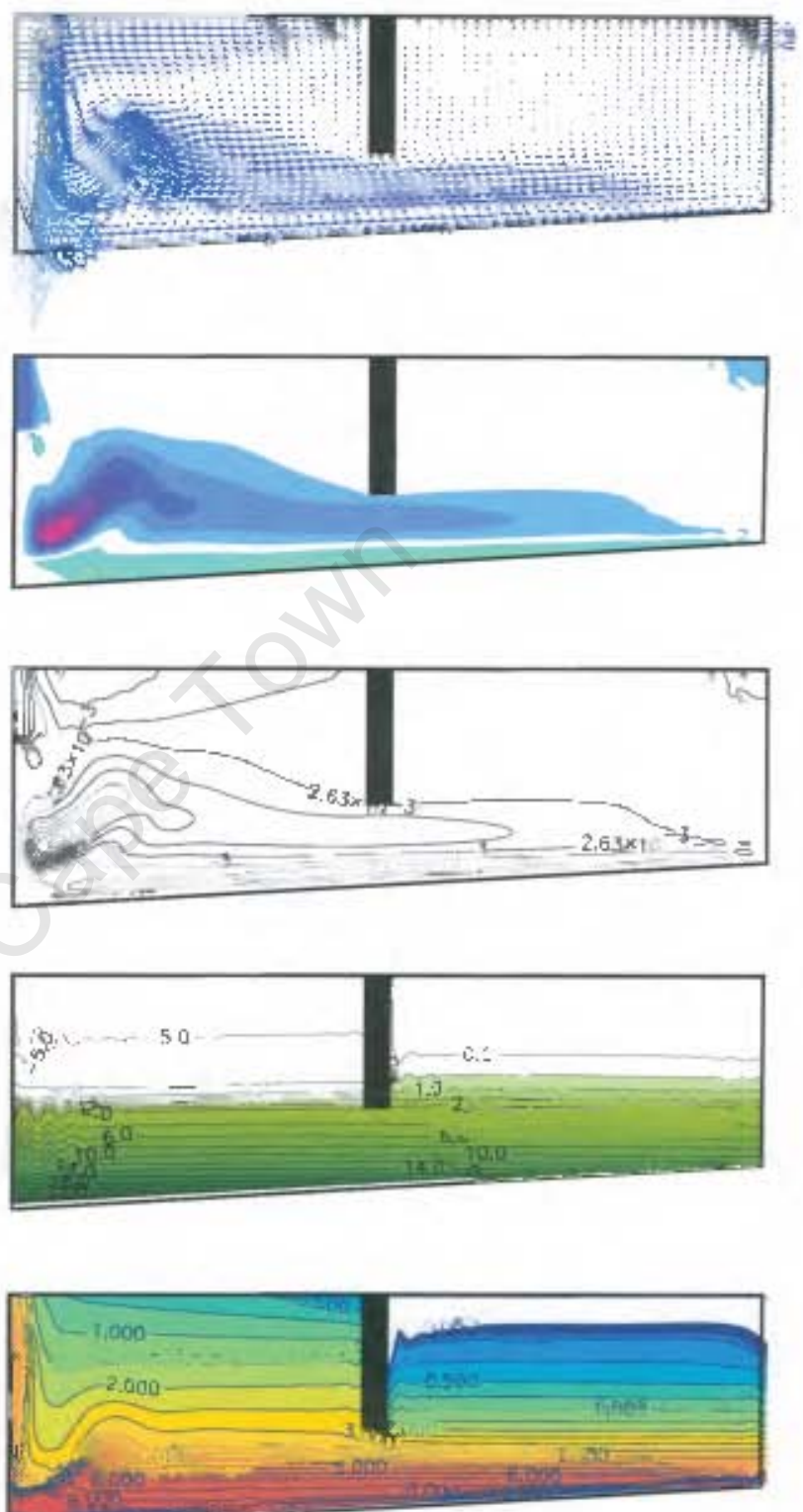

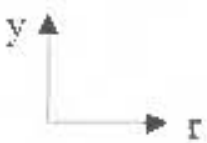

Figure 6.27: Watts tests 4 and 12: Steady state case, predicted flow pattern (a), horizontal velocity $u$ in $\mathrm{m} / \mathrm{s}(\mathrm{b}, \mathrm{c})$, pressure field in $\mathrm{Pa}$ (d) and sludge distribution $c$ in $g / \ell(e)$. 


\begin{tabular}{|c|c|c|c|c|c|}
\hline Parameter & & & $\begin{array}{l}\text { Darvill } \\
\text { old tank } \\
\text { Test } 3\end{array}$ & $\begin{array}{l}\text { Darvill } \\
\text { old tiank } \\
\text { Test } 3+ \\
13.3 \%\end{array}$ & $\begin{array}{l}\text { Darvill } \\
\text { new tank } \\
\text { Test } 3+ \\
33.3 \%\end{array}$ \\
\hline SST & Surface area & $\mathrm{m}^{2}$ & 962 & 962 & 962 \\
\hline $\begin{array}{l}\text { Loading } \\
\text { conditions }\end{array}$ & $\begin{array}{l}\text { Influcnt flow } \\
\text { Recycle flow } \\
\text { Recycle ratio } \\
\text { Feed } \\
\text { concentration }\end{array}$ & $\begin{array}{l}\mathrm{m}^{3} / \mathrm{h} \\
\mathrm{m}^{3} / \mathrm{h} \\
\mathrm{g} / \ell\end{array}$ & $\begin{array}{l}948 \\
750 \\
0.79 \\
3.6\end{array}$ & $\begin{array}{l}1074 \\
750 \\
0.7 \\
3.6\end{array}$ & $\begin{array}{l}1074 \\
750 \\
0.7 \\
3.6\end{array}$ \\
\hline $\begin{array}{l}\text { Sludge } \\
\text { settleatrility }\end{array}$ & $\begin{array}{l}\mathrm{V}_{0} \\
\mathrm{n}_{4} \\
\text { DSVI }\end{array}$ & $\begin{array}{l}\mathrm{m}^{3} / \mathrm{h} \\
\mathrm{m} \ell / \mathrm{g}\end{array}$ & $\begin{array}{l}8.0 \\
0.43 \\
62 \\
\end{array}$ & $\begin{array}{l}8.0 \\
0.43 \\
62 \\
\end{array}$ & $\begin{array}{l}8.1) \\
0.43 \\
62\end{array}$ \\
\hline Retention time & t & $h_{1}$ & 1.3 & 1.4 & 2.3 \\
\hline Time step & $\Delta \mathrm{t}$ & $s$ & 5 & 5 & $\overline{5}$ \\
\hline $\begin{array}{l}\text { Turbulent } \\
\text { viscosity }\end{array}$ & $\nu_{t}$ & $\mathrm{~m}^{2} / \mathrm{s}$ & 0.0005 & 0.0005 & 0.0005 \\
\hline $\begin{array}{l}\text { Schmidt } \\
\text { number }\end{array}$ & $\sigma_{\mathrm{a}}$ & & 5 & $\bar{y}$ & 5 \\
\hline
\end{tabular}

Table 6.1: Summary of the loading conditions and model parameter uscd for the simulation of the Darvill old and now tanks. 


\begin{tabular}{|c|c|c|c|c|c|}
\hline Parameter & & & $\begin{array}{l}\text { Darvill } \\
\text { old tank } \\
\text { Test } 3\end{array}$ & $\begin{array}{l}\text { Darvill } \\
\text { old tank } \\
\text { Test } 3+ \\
13.3 \%\end{array}$ & $\begin{array}{l}\text { Darvill } \\
\text { new tank } \\
\text { Test } 3+ \\
13.3 \%\end{array}$ \\
\hline $\begin{array}{l}\text { Loading } \\
\text { conditions }\end{array}$ & $\begin{array}{l}\text { Influent flow } \\
\text { Recycle flow } \\
\text { Recycle ratio } \\
\text { Feed } \\
\text { concentration }\end{array}$ & $\begin{array}{l}\mathrm{m}^{3} / \mathrm{h} \\
\mathrm{m}^{3} / \mathrm{h} \\
\mathrm{g} / \ell\end{array}$ & $\begin{array}{l}948 \\
750 \\
0.79 \\
\\
3.6\end{array}$ & $\begin{array}{l}1074 \\
750 \\
0.7 \\
3.6\end{array}$ & $\begin{array}{l}1074 \\
750 \\
0.7 \\
3.6\end{array}$ \\
\hline $\begin{array}{l}\text { Sludge } \\
\text { settleability } \\
\text { Retention time }\end{array}$ & $\begin{array}{l}\mathrm{V}_{o} \\
\mathrm{n}_{4} \\
\mathrm{t}\end{array}$ & $\begin{array}{l}\mathrm{m}^{3} / \mathrm{h} \\
\mathrm{h}\end{array}$ & $\begin{array}{l}8.0 \\
0.43 \\
1.4\end{array}$ & $\begin{array}{l}8.0 \\
0.43 \\
1.3\end{array}$ & $\begin{array}{l}8.0 \\
0.43 \\
2.3\end{array}$ \\
\hline $\begin{array}{l}\text { Recycle } \\
\text { concentration }\end{array}$ & $c_{R A S}$ & $\mathrm{~g} / \ell$ & 8.2 & 8.8 & 8.76 \\
\hline Mass balance & 68 & $\%$ & 101 & 101.5 & 100 \\
\hline Observed result & Experiment & & Safe & Not tested & Not tested \\
\hline $\begin{array}{l}\text { Test result } \\
\mathrm{ESS}>50 \mathrm{mg} / \ell\end{array}$ & $\begin{array}{l}\text { SettlerCAD } \\
\text { (WRC Report } \\
\text { No. } 835 / 1 / 02 \text { ) }\end{array}$ & & Safe & Fail & Safe \\
\hline $\begin{array}{l}\text { Test result } \\
\text { ESS }>50 \mathrm{mg} / \ell\end{array}$ & FEM code & & Safe & Fail & Safe \\
\hline
\end{tabular}

Table 6.2: Summary and comparison of computational and benchmark results for the simulation of Darvill old and new tanks. 


\begin{tabular}{|c|c|c|c|c|c|}
\hline Parameter & & & $\begin{array}{l}\text { Watts } \\
\text { Test } 1\end{array}$ & $\begin{array}{l}\text { Watts } \\
\text { Test } 4\end{array}$ & $\begin{array}{l}\text { Watts } \\
\text { Test } 12\end{array}$ \\
\hline SST & Surface area & $\mathrm{m}^{2}$ & 659 & 659 & 659 \\
\hline $\begin{array}{l}\text { Loading } \\
\text { conditions }\end{array}$ & $\begin{array}{l}\text { Influent flow } \\
\text { Recycle flow } \\
\text { Recycle ratio } \\
\text { Feed } \\
\text { concentration }\end{array}$ & $\begin{array}{l}\mathrm{m}^{3} / \mathrm{h} \\
\mathrm{m}^{3} / \mathrm{h} \\
\mathrm{g} / \ell\end{array}$ & $\begin{array}{l}475.1 \\
397.5 \\
0.84 \\
4.053\end{array}$ & $\begin{array}{l}788.9 \\
397.5 \\
0.51 \\
4.130\end{array}$ & $\begin{array}{l}1095.8 \\
397.5 \\
0.36 \\
\\
3.444\end{array}$ \\
\hline $\begin{array}{l}\text { Sludge } \\
\text { settleability }\end{array}$ & $\begin{array}{l}\mathrm{V}_{0} \\
\mathrm{n}_{4} \\
\text { DSVI }\end{array}$ & $\begin{array}{c}\mathrm{m}^{3} / \mathrm{h} \\
\mathrm{m} \ell / \mathrm{g}\end{array}$ & $\begin{array}{l}7.62 \\
0.3055 \\
94\end{array}$ & $\begin{array}{l}7.62 \\
0.3055 \\
94\end{array}$ & $\begin{array}{l}7.62 \\
0.3055 \\
94\end{array}$ \\
\hline Retention time & t & h & 3.0 & 2.2 & 1.8 \\
\hline Timestep & $\Delta \mathrm{t}$ & $\mathrm{s}$ & 10 & 10 & 10 \\
\hline $\begin{array}{l}\text { Turbulent } \\
\text { viscosity }\end{array}$ & & $\mathrm{m}^{2} / \mathrm{s}$ & $\mathrm{k}-\varepsilon$ model & $k-\varepsilon$ model & $\mathrm{k}-\varepsilon$ model \\
\hline $\begin{array}{l}\text { Schmidt } \\
\text { numbers } \sigma_{s}\end{array}$ & $\begin{array}{l}c \leq 1 \mathrm{~g} / \ell \\
c \leq 8 \mathrm{~g} / \ell \\
8 \mathrm{~g} / \ell<\mathrm{c}\end{array}$ & & $\begin{array}{l}1.0 \\
1.5 \times c \\
2.0 \times c\end{array}$ & $\begin{array}{l}1.0 \\
1.5 \times c \\
2.0 \times c\end{array}$ & $\begin{array}{l}1.0 \\
1.5 \times c \\
2.0 \times c\end{array}$ \\
\hline
\end{tabular}

Table 6.3: Summary of the loading conditions and model parameter used for the simulation of the Watts tanks. 


\begin{tabular}{|c|c|c|c|c|c|}
\hline Parameter & & & $\begin{array}{l}\text { Watts } \\
\text { Test } 1\end{array}$ & $\begin{array}{l}\text { Watts } \\
\text { Test } 4\end{array}$ & $\begin{array}{l}\text { Watts } \\
\text { Test } 12\end{array}$ \\
\hline $\begin{array}{l}\text { Loading } \\
\text { conditions }\end{array}$ & $\begin{array}{l}\text { Influent flow } \\
\text { Recycle flow } \\
\text { Recycle ratio } \\
\text { Feed } \\
\text { concentration }\end{array}$ & $\begin{array}{l}\mathrm{m}^{3} / \mathrm{h} \\
\mathrm{m}^{3} / \mathrm{h} \\
\mathrm{g} / \ell\end{array}$ & $\begin{array}{l}475.1 \\
397.5 \\
0.84 \\
\\
4.053\end{array}$ & $\begin{array}{l}788.9 \\
397.5 \\
0.51 \\
4.130\end{array}$ & $\begin{array}{l}1095.8 \\
397.5 \\
0.36 \\
\\
3.444\end{array}$ \\
\hline $\begin{array}{l}\text { Sludge } \\
\text { settleability } \\
\text { Retention time }\end{array}$ & $\begin{array}{l}\mathrm{V}_{0} \\
\mathrm{n}_{4} \\
\mathrm{t}\end{array}$ & $\begin{array}{l}\mathrm{m}^{3} / \mathrm{h} \\
\mathrm{h}\end{array}$ & $\begin{array}{l}7.62 \\
62 \\
3.0\end{array}$ & $\begin{array}{l}7.62 \\
62 \\
2.2\end{array}$ & $\begin{array}{l}7.62 \\
62 \\
1.8\end{array}$ \\
\hline $\begin{array}{l}\text { Recycle } \\
\text { concentration }\end{array}$ & $c_{R A S}$ & $\mathrm{~g} / \ell$ & 8.2 & 11.9 & 11.6 \\
\hline Mass balance & 60 & $\%$ & 92.7 & 97.5 & 100 \\
\hline Observed result & Experiment & & Safe & Safe & Fail \\
\hline $\mathrm{ESS}>50 \mathrm{mg} / \ell$ & $\begin{array}{l}\text { SettlerCAD } \\
\text { (WRC Report } \\
\text { No. } 835 / 1 / 02 \text { ) }\end{array}$ & & Safe & Safe & Fail \\
\hline $\mathrm{ESS}>50 \mathrm{mg} / \ell$ & FEM code & & Safe & Safe & Fail \\
\hline
\end{tabular}

Table 6.4: Summary and comparison of computational and benchmark results for the simulation of the Watts tanks. 


\section{Chapter 7}

\section{Conclusions, and scope of future research}

\subsection{Opening remarks}

This thesis has been concerned with the development, testing and application of a finite element code for the two-dimensional numerical modelling and simulation of flows in secondary settling tanks. The goal has been to develop a model that is capable of simulating the benchmark results available in the literature. This 'real-life' application requires the design of high-performance, accurate and robust algorithms. In this chapter these tools are reviewed; and the results, achievements and unresolved topics are discussed.

\subsection{Motivations and findings}

In wastewater treatment plants based on activated sludge the secondary settling tank (SST) forms a crucial part. Besides having to produce the separation of activated sludge and clarified effluent, the secondary settling tank is used to concentrate and recycle the settled sludge to the biological reactor. The biological reactor tank and SST are strongly linked together and form a functional design and operational unit. The efficiency of the biological reactor in the wastewater treatment system can be determined through the efficiency of this final clarifying process. Furthermore, compliance with the effluent requirements strongly 
depends on the efficiency of the secondary settling tank.

Hydrodynamic models have been developed for simulating secondary settling tanks (SST) to get a better understanding of the complex flow patterns in these tanks and to make design and optimization of the SST internal features possible. In this thesis the development of a 2D hydrodynamic SST model is presented that is based on the finite element method (FEM). With regard to secondary settling tanks the flexible geometry of the FEM supports the description and adaptation of complex internal features, such as inlet and outlet arrangements.

The performance of a settling tank is determined by the tank hydraulics, the transport and removal of solids, and by turbulence effects. During the development of the FEM program it was observed that all variables are strongly coupled both physically, and through the governing equations. In addition, the treatment of the gravity-density term in the momentum equations has been an important issue to be addressed. The flow pattern in SSTs strongly depends on the viscosity and on buoyancy forces. Numerical instability additionally appears due to the increasing influence of the buoyancy source term.

These complexities pose great challenges in respect of the numerical and computational strategies to be used. A combination of successful numerical techniques was chosen in order to arrive at a FEM code that fulfils the various basic requirements:

- Owing to the strong link between all variables, the use of an equal interpolation for all variables was chosen in order to connect them at the same nodal points. However, the four-noded bilinear quadrilateral element which was used, does not satisfy the Babuska-Brezzi condition, resulting in a checkerboard pressure mode in conventional applications. This difficulty has been successfully resolved by circumventing the BabuskaBrezzi restriction through proper embedding of the spatial discretization in the time-discretization process. This approach leads to a system that is positive-definite, and which gives a unique discrete solution.

- The solution of the coupled equation set of continuity- and momentum is achieved through a time-splitting algorithm. The combination of two projection schemes, namely the projection scheme of Van Kan and of Chorin, is applied in order to unite the better accuracy and stability of Van Kan's second-order accurate pressure correction scheme, and the feature of 
Chorin's velocity projection scheme, which circumvents the Babuška-Brezzi restriction. Chorin's projection scheme works well for neutral density flows, but due to its first-order accuracy it shows instabilities when hydrostatic pressure becomes an increasing influence, which is always the case in SSTs. The time step has to be chosen very small, which would have made an economical investigation of the long time behaviour of SSTs impossible. Van Kan's projection scheme, on the other hand, is of second-order accuracy and is stable even for very high concentration (high hydrostatic pressure) and large time steps, but it leads to spurious pressure modes. To combine the advantages of both time-splitting schemes Van Kan's algorithm has been used to calculate the long-time behaviour, and use is made of Chorin's algorithm in order to eliminate the checkerboard pressure.

- For time-integration the fractional-step $\theta$-scheme (FS) has been successfully used. This time integration scheme combines the advantages of both the backward Euler scheme and the Crank-Nicolson schemes. It divides the time step into three time sub-steps, and its special settings for each substeps leads to strong A-stability and second-order accuracy.

- The system of equations is successfully decoupled through a sequential approach, with the partitioning into the three subsets of velocity components and pressure, concentration, and of turbulent kinetic energy and its dissipation. The numerical solution process is split into two parts: the inner and the outer iterations. At each inner iteration the source terms and the coefficients that depend on the other variables are kept fixed. At the outer iterations the coefficient matrices and the source vector must be updated and the process repeated to obtain a solution that satisfies all of the equar tions. For the optimization of the solution process the right number of inner iterations per outer iteration is crucial.

- Nonstationary iteration methods, namely, the conjugate gradient (CG) and generalized minimal residual (GMRES) methods, are used to solve the resulting symmetric and positive-definite linear problem. The CG and GMRES demonstrate efficiency and speed and they provide a reference for checking convergence.

For the validation of SST performance the code has been used to simulate the behaviour of full-scale SSTs in two dimensions. These results have been compared 
against experimental data obtained from tests on real secondary settling tanks, and against the computational results obtained with the 2D hydrodynamic SST simulation package SettlerCAD.

The finite element code gives reasonable results and is in good agreement with the experimental data, as well as with the computational results of SettlerCAD. Good results could also be obtained with the use of a constant turbulent viscosity, with the requirement that the inlet region is small compared to the settling section of the SST.

However, the computations also show that

1. the simulation results react very sensitively to the magnitude of turbulence;

2. due to the fact that the laminar and turbulent diffusivity of the activated sludge is of an unknown magnitude, it has to be adjusted in order to achieve the same results as observed in the experiments;

3. with the necessary and inevitable simplifications of $2 \mathrm{D}$ models mass balance cannot always be achieved;

4. the assumption that the turbulent diffusivity is proportional to the turbulent viscosity, cannot be confirmed. The best computational results were obtained by using a constant and small diffusion coefficient, so that the sludge is less influenced by diffusion than assumed by using the Reynolds analogy;

5. very high recycle sludge concentration cannot be achieved because of the still unknown behaviour of activated sludge in the high concentration environment, which necessarily yields no mass balance;

6. sludge compaction as a result of hydrostatic pressure at the bottom of the tank cannot be considered due to the lack of appropriate data and the fact that the mathematical model does not incorporate this effect.

As a result of these uncertainties and the significant effect of turbulence on the settling process, the computational outcome does not provide a definitive answer. To properly validate the model further information and calibration are needed.

The correct modelling of the activated sludge settleability is essential for the correct modelling of secondary settling tanks. The hydrodynamic models for SSTs 
rely on experimental data that characterize the sludge behaviour in the four regimes of particle settlement, namely discrete, flocculent, zone and compaction settling. While the zone settling velocity can be measured for concentrations $>1000 \mathrm{mg} / \ell$, no information can be obtained for the dilute and flocculation regime due to the non-settleability of particles in the low concentration range. But low concentration settling behaviour of flocculent dispersed particles is especially important for water treatment plant settling tanks where the feed concentration is usually below zone settling concentrations. Flocculation has a profound influence on the effluent suspended solids concentration. The inability to measure the settling velocity for low concentrations is therefore a weakness in CFD modelling of secondary settling tanks.

Furthermore, the hydrostatic pressure leads to compaction and promotes high accumulation at the bottom of the tank, which is required for loading conditions with low recycle ratio. Due to the lack of knowledge about the situation in the compaction stage, which also includes a different viscosity behaviour, these phenomena cannot be included in the CFD models, which in turn makes the model inappropriate for the simulation of SSTs with low recycle ratio.

At the same time very little is known about the water-solids interaction, and due to the problems and uncertainties mentioned above concerning the behaviour of activated sludge in the different concentration ranges, the question arises as to whether the two phases, pure water and activated sludge, should be modelled separately by using a two-phase model in which conservation equations are set up for each phase. This of course would lead to a highly nonlinear model with a considerable number of unknowns and would require increased computation time.

In summary, the finite element formulation developed and presented here is able successfully to simulate complex flows in SSTs. The code is stable for coarse meshes and relatively large time steps, and a local refinement is very easy to construct due to the possibility of using different elements. The variation of the results is less an outcome of uncertainties within the numerical modelling procedure, than due to a lack of appropriate and reliable input data. Further experimental investigation is required with a view to improving hydrodynamic models for secondary settling tanks.

Emanating from the research undertaken of this thesis are several computational 
issues that have not been completely resolved in this study, and which could be subject of further research. The computational time, and with it the computational efficiency, can be improved with the use of numerical techniques, such as

- preconditioning for the CG and GMRES methods;

- alternative iterative treatments;

- the implementation of a multigrid technique;

- another element pair, which combines accuracy, efficiency and passes the Babuskka-Brezzi restriction;

- the use of compressed sparse row storage in order to save computation time; and

- the implementation of user-friendly pre- and postprocessing procedures. 


\section{Chapter 8}

\section{References}

Adams E.W. and Rodi W. (1990) Modelling flow and mixing in sedimentation tanks. J. Hydraul. Eng., ASCE, 116 (7), 895-913.

Anderson H.M. (1981) A dynamic simulation model for wastewater renovation systems. Ph.D. thesis, Wayne State University, Detroit, Michigan, USA.

Anderson N.E. (1945) Design of settling tanks for activated sludge. Sewage Works J., 17(1), 50-63.

Armaly B.F., Durst F., Perera J.C.F and Schönung, B (1983) Experimental and theoretical investigation of backward-facing step flow. J. Fluid. Mech., 127, $473-496$.

Armbruster M., Krebs P. and Rodi W. (2000) Numerical modelling of dynamic sludge blanket behaviour in secondary clarifiers. Procs. 1st World Water Congress of IWA, Book 3, 308-315, Paris, France.

ATV (Abwassertechnische Vereinigung) (1991) Dimensioning of single stage activated sludge plants upwards from 5000 total inhabitants and population equivalents. ATV Rules and Standards. Wastewater-waste, UDC 628.356:629.32.-0-1.2(083), A131, Issue No. 11/92.

ATV (Abwassertechnische Vereinigung) (1993) Anwendungshinweise zum ATV Arbeitsblatt A 131 - Teil Nachklärbecken, Arbeitsblatt des Arbeitsausschusses 2.5 - Absetzverfahren. Korrespondenz Abwasser 40(2), 224-225. 
Babuska I. (1973) The finite element method with Lagrange multipliers. Num. Math., 20, 179-192, also: (1971) Error bound for finite element methods. Num. Math.,16, 322-333.

Barett R., Berry M., Chan T.F., Demmel J., Donato J.M., Dongarra J., Eijkhout V., Pozo R., Romine C. and Van der Vorst H. (1994) Templates for the solution of linear systems: building blocks for iterative methods. SIAM, Philadelphia, PA, USA.

Baumer P., Volkert P. and Krebs P. (1996) Dynamic modelling tests for final settling tanks. Wat. Sci. Technol., 34 (3/4), 267-274.

Bokil S.D. and Bewtra J.K. (1972) Influence of mechanical blending on aerobic digestion of waste activated sludge. 6th Int. Conf. on Water Pollution Research, Proc. 421-438, Jerusalem, Israel.

Boussinesq J. (1897) Thérie de l'ecoulement tourbillonant. Paris.

Bretcher U., Hager W. and Hager W.H. (1984) Untersuchungen über die Strömungsund Feststoff-Verteilung in Nachklärbecken. GWF-Wasser/Abwasser, 125 (2), 81-90.

Bretcher U., Krebs P. and Hager W.H. (1992) Improvement of flow in final settling tanks. J. Environ. Eng., ASCE, 118 (3), 307-321.

Brezzi F. (1974) On the existence, uniqueness and approximation of saddle point problems arising from lagrangian multipliers. R.A.I.R.O., 8-R2, 129-151.

Brooks A.N. and Hughes T.J.R. (1982) Streamline Upwind / Petrov Galerkin formulations for convection dominated flows with particular emphasis on the incompressible Navier Stokes equations. Comp. Meth. Appl. Mech. Eng., 32, 199-259.

Burnett D.S. (1987) Finite element analysis. Addison-Wesley (Reading Mass.)

Camp T.R. (1946) Sedimentation and the design of settling tanks. Trans., ASCE, 5 (11), 895-936.

Caretto L.S., Curr R.M. and Spalding D.B. (1972) Two numerical methods for three-dimensional boundary layers. Comput. Methods Appl. Mech. Eng, 1,39 . 
Clough R.W. (1960) The finite element in plane stress analysis. Proc. 2nd ASCR Conf. on Electronic Computation. Pittsburgh, Pa., Sept. 1960.

Coe H.S. and Clevenger G.H. (1916) Methods for determining the capacities of slime settling tanks. Trans. Am. Inst. Mining Eng., 55, 356-383.

Crouzeix M. and Raviart P.A. (1973) Conforming and non-conforming finite element methods for solving the stationary Stokes equations, R.A.I.R.O. R-3, 77-104.

Dahl C.P., Larsen T. and Peterson O. (1994) Numerical modelling and measurement in a test secondary settling tank. Wat. Sci. Technol., 30 (2), 219-228.

De Haas D.W. (1998) The use of simultaneous chemical precipitation in modified activated sludge systems exhibiting enhanced biological phosphate removal. $\mathrm{PhD}$ thesis, University of Cape Town, South Africa, (Chapter 6 - Fullscale plant trial).

Deininger A. (1997) Geschwindigkeits- und Feststoffverteilung in radial durchströmten Nachklärbecken. Bericht 131, Institut für Wassergüte und Abfallwirtschaft, TU Munchen, Germany.

DeVantier B.A. and Larock B.E. (1986) Modelling a recirculation density-driven turbulent flow. Int. J. Num. Meth. Fluids, 6 (4), 241-253.

DeVantier B.A. and Larock B.E. (1987) Modelling sediment-induced density current in sedimentation basins. J. Hydraul. Eng., ASCE, 113 (1), 80-94.

Dick R.I. and Ewing B. (1967) The rheology of activated sludge. J. Water Pollution Control Federation, 39 (40), 543-560.

Diehl I.G.and Jeppsson U. (1996) An evaluation of a dymamic model of secondary clarifier. Wat. Sci. Technol., 34 (5/6), 19-26.

Dold P.L., Wentzel M.C., Billing A.E., Ekama G.A. and Marais G.v.R. (1991) Activated sludge simulation models, version 1.0. Water Research Commission, P.O. Box 824, Pretoria, 0001, RSA.

Donea J., Giuliani S. and Laval H. (1982) Finite element solution of the unsteady Navier-Stokes equation by fractional step method. Comp. Meth. Appl. Mech. Eng., 30, 53-73. 
Dunbar W. (1954) Leitfaden für die Abwasserreinigungsfrage. 3rd ed. Verlag R. Oldenbourg, München, Germany.

Dupont R. and Henze M. (1992) Modelling of the secondary clarifier combined with the activated sludge model No. 1., Wat. Sci. Technol., 25 (6), 285-300.

Ekama G.A. and Marais G.v.R (1984) Two improved sludge settleability parameters. IMIESA, 9 (6), 285-300.

Ekama G.A. and Marais G.v.R (1986) Sludge settleability and secondary settling tank design procedures. Wat. Pollut. Control, 85 (1), 101-113.

Ekama G.A., Barnard J.L., Gunthert F.W., Krebs P., McCorquodale J.A., Parker D.S. and Wahlberg E.J., (1997) Secondary settling tanks: theory, modelling, design and operation. IAWQ STR No 6, International Association on Water Quality, London.

Ekama G.A. and Marais P. (2002) Hydrodynamic modelling of secondary settling tanks: Part 1. WRC Report No. 835/1/02, Water Research Commission, South Africa.

Fletcher C.A.J. (1984) Computational Galerkin Methods. Springer Verlag, Berlin.

Franca L.P., Frey S.L. and Hughes T.J.R. (1992) Stabilized finite element methods: I. Application to the advective-diffusive model. Comp. Meth. Appl. Mech. Eng., 95, 253-276.

Franca L.P. and Frey S.L. (1992) Stabilized finite element methods: II. The incompressible Navier-Stokes equations. Comp. Meth. Appl. Mech. Eng., 99, 209-233.

Galerkin, B.G. (1915) Problems of elastic stability of rods and plates. Eng. Bull., 19, 897-908.

Gartling D.K. (1990) A test problem for outflow boundary conditions-flow over a backward facing step. Int. J. Num. Meth. Fluids, 11, pp. 953.

Ghia U., Ghia K.N. and Shin C.T. (1982) High-Re solutions for incompressible flow using the Navier-Stokes equations and a multigrid method. J. Comput. Phys., 48, 387-411. 
Ghia K.N., Oswald G.A. and Ghia U. (1989) Analysis of incompressible massively separated viscous flows using unsteady Navier-Stokes equations. Int. J. Num. Meth. Fluids, 9, pp. 1025.

Gidaspow D. (1994) Multiphase Flow and Fluidization. Academic Press, Inc., San Diego.

Glowinski R. and Periaux J. (1987) Numerical methods for nonlinear problems in fluid dynamics. Proc. Intern. Seminar on Scientific Supercomputers, Paris, Feb. 2-6.

Gosman A.D. and Pun W.M. (1974) Lecture notes for calculation of recirculating flows. HTS/74/2, Imperial College, London, UK.

Günthert F.W. and Deininger A. (1995) Final settling tanks - New aspects and design approaches. Procs 7th IAWQ Conference on design and Operation of Large WWTPS, Vienna, Austria.

Hazen A. (1904) On sedimentation. Trans. ASCE, 53, 45-71.

Härtel L. and Pöpel H.L. (1992) A dynamic secondary clarifier model including processes of sludge thickening. Wat. Sci. Technol., 25 (6), 267-284.

Henze M., Grady C.P.L. Jr., Gujer W., Marais G.v.R. and Matsuo T. (1987) Activated sludge model No. 1. (IAWQ STR No. 1), IAWQ, London, UK.

Hestenes M.R. and Steifel E. (1952) Methods of conjugate gradient for solving linear systems. J. of Res. Nat. Bureau Standards, 49,609-436.

Hill D.L. and Baskharone E.A. (1997) Development of a multiblock pressurebased algorithm using mixed interpolation for turbulent flows. Int. J. Num. Meth. Fluids, 25, 615-631.

Hughes T.J.R. (1987) The finite element method. Prentice-Hall, Inc., Englewood Cliffs, New Jersey.

Kardestuncer H. (ed.) (1987) Finite element handbook. McGraw-Hill, New York.

Kelly C.T. (1995) Iterative methods for linear and nonlinear equations. SIAM Frontiers in Applied Mathematics. 
Kolmogorov A.N. (1942) Equations of turbulent motion of an incompressible fluid. Izv. Akad. Nauk. SSR, Seria fizicheska Vi., No. 1-2, 56-58.

Koopman B.L. and Cadee K. (1983) Prediction of thickening capacity using diluted sludge volume index. Wat. Res., 17 (10), 1427-1431.

Krebs P. (1989) Modellierung von Strömungen in rechteckigen Nachklärbecken. Gas Wasser Abwasser/Abwasser 69 (11), 718-728.

Krebs P. (1991a) The hydraulics of final settling tanks. Wat. Sci. Technol., 23 (4-6), 1037-1046.

Krebs P. (1991b) Modellierung und Verbesserung der Strömung in Nachklärbecken. ETH-Dissertation 9486, Report 157, Environment Protection Agency, Bern, Switzerland.

Krebs P.(1995) Success and shortcomings of clarifier modelling. Wat. Sci. Technol., 31 (2), 181-191.

Krebs P., Stamou A.I., Gracía-Heras J.L. and Rodi W. (1996) Influence of inlet and outlet configuration on the flow in secondary clarifieres. Wat. Sci. Technol., 34 (5/6), 1-9.

Kynch J.J. (1952) A theory of sedimentation. Trans. Faraday Soc., 148, 166176.

Lai W.M., Rubin D. and Krempl E. (1993) Indroduction to continuum mechanics. Pergamon, Oxford.

Lakehal D., Krebs P., Krijgsman J. and Rodi W. (1999). Computing shear flow and sludge blanket in secondary clarifiers. J. Hydraulic Eng., ASCE, 125 (3), 253-262.

Larsen P. (1977) On the hydraulics of rectangular settling basins. Report No. 1001, Department of Water Research Engineering, Lund Institute of Technology, University of Lund, Sweden.

Launder B.E. and Spalding D.B. (1974) The numerical computation of turbulent flow., Comp. Meth. Appl. Mech. Eng., 3, pp. 269.

Lucquin B. and Pironneau O. (1998) Indroduction to scientific computing. John Wiley, New York. 
Lyn D.A. (1992) Laboruntersuchungen der Strömungsverhältnisse in einem längsdurchströmten Modellabsetzbecken. Report No. SFB210/T/82, pp. 45-65, University of Karlsruhe, Karlsruhe, Germany.

Lyn D.A., Stamou A.I. and Rodi W. (1992) Density currents and shear-induced flocculation in sedimentation tanks. J. Hydraul. Eng., ASCE, 118 (6), $849-867$.

Majumdar S. (1986) Development of a finite volume procedure for prediction of fluid flow problems with complex irregular boundaries. Report SFB 210/T/29, University of Karlsruhe, Karlsruhe, Germany.

McCorquodale J.A. and Zhou S. (1993). Effects of hydraulics and solids loading on clarifier performance. J. Hydraulic Res:, 31 (4), 461-478.

Merkel W. (1971) Untersuchungen über das Verhalten des belebten Schlammes im System Belebtschlamm-Nachklärbecken. Gewässerschutz, Wasser-Abwasser, 5, D82, Institut für Siedlungswasserwirtschaft, Technische Hochschule Aachen, Aachen, Germany.

Mohlman F.W. (1934) The sludge index. Sewage Wks. J. 6, 119-122.

Muller-Urbaniak S. (1993) Eine Analyse des Zwischenschritt- $\theta$-Verfahrens zur Lösung der instationären Navier-Stokes Gleichungen, $\mathrm{Ph} . \mathrm{D}$. thesis, University Heidelberg. Heidelberg, Germany.

Newmark, N.M. (1949), Numerical methods of analysis in bars, plates and elastic bodies. Numerical Methods in Analysis in Engineering (ed. L.E. Grinter), Macmillan.

Nikuradse J. (1932) VDI-Forschungsheft Nr. 356.

Oden J.T. (1972) Finite elements of nonlinear continua. McGraw-Hill, New York.

Otterpohl R. and Freund M. (1992) Dynamic models for clarifiers of activated sludge plants with dry and wet weather flow. Wat. Sci. Technol., $26(5 / 6)$, 1391-1400.

Ozinsky A.E., Ekama G.A. and Reddy B.D. (1994) Mathematical simulation of dynamic behaviour of secondary settling tanks. Research Report W85, 
Department of Civil Engineering, University of Cape Town, Rondebosch, RSA.

Parker D.S., Kaufman W.J. and Jenkins D. (1971) Physical conditioning of the activated sludge floc. Journal WPCF, 43(9), 1817-1833.

Pflanz P. (1969) Performance of activated sludge secondary sedimentation basins. In Proceedings of the 4th IAWPR International conference, Prague, 569593, Pergamon Press, Oxford.

Pitman A.R. (1984) Settling of nutrient removal activated sludge. Wat. Sci. Technol., 17, 493-504.

Prandt1 L. (1925) Über ein neues Formelsystem für die ausgebildete Turbulenz. Nachr. Akad. Wiss., Göttingen, Math.-Phys. Klasse, pp. 6.

Rannacher R. and Turek S. (1992) A simple nonconforming quadrilateral Stokes element. Numer. Meth. Part. Diff. Equ., 8, 97-111.

Rannacher R. (1993) Numerical analysis of the Navier-Stokes equations. Appl. Math., 38, 361-380.

Rannacher R., Müller S., Prohl A. and Turek S. (1994) Implicit time-discretization of the nonstaionary incompressible Navier-Stokes equations. Proc. 10th GAMM-Seminar, Kiel, January 14-16, (G. Wittum, W. Hackbusch, eds.), Vieweg.

Rayleigh J.W.S. (1877) Theory of Sound. 1st re. ed., Dover Publishers, New York.

Reddy B.D. (1998) Introductory functional analysis with applications to boundary value problems and finite elements. Texts in Applied Mathematics 27, Springer Verlag, New York.

Reddy J.N. (1984) An introduction to the finite element method. McGraw-Hill, New York.

Reynolds O. (1900) Proc. Lit. and Philos. Soc. Manchester, 12 (1874/75) Papers on Mechanical and Physical Subjects, Vol. I, pp. 81.

Rice J.G. and Schnipke R.J. (1986) An equal-order velocity-pressure formulation that does not exhibit spurious pressure modes. Comp. Meth. Appl. Mech. Eng., 58, 135-149. 
Ritz W. (1909) Über eine neue Methode zur Lösung gewisser Variations-Probleme der mathematischen Physik. J. Reine Angew. Math., 135, 1-61.

Rodi W., Majumdar S. and Schönung B. (1989) Finite volume method for twodimensional incompressible flows with complex boundaries. Comp. Meth. Appl. Mech. Eng., 75, 369-392.

Rodi W. (1993) Turbulence and their application in hydraulics. 3rd ed., Balkema, Rotterdam, Netherlands.

Rosten H.I. and Spalding D.B. (1987) The PHOENICS reference manual. CHAM Ltd., London, Report TR 200.

Saad Y. and Schultz M.H. (1986) GMRES: A generalized minimal residual method for solving nonsymmetric linear systems. SIAM J. Sci. Statist. Comput., 7 , 856-869.

Sani R.L., Gresho P.M., Lee R.L. and Griffiths D.F. (1981) The cause and cure (?) of the spurious pressures generated by certain FEM solutions of the incompressible Navier-Stokes equations: Part 1. Int. J. Num. Methods Fluids 1, 17-43.

Sani R.L., Gresho P.M., Lee R.L. and Griffiths D.F. (1981) The cause and cure (!) of the spurious pressures generated by certain FEM solutions of the incompressible Navier-Stokes equations: Part 2. Int. J. Num. Methods Fluids 1, 171-204.

Schamber D.R. and Larock B.E. (1981) Numerical analysis of flow in sedimentation basins. J. Hydraul. Div., ASCE, 107, (HY5), 575-591.

Simpson J.E. (1987) Gravity currents in the environment and the laboratory. Ellis Horwood Ltd., Chichester.

Smith I.M. and Griffiths D.V. (1998) Programming the finite element method. 3rd ed., John Wiley, New York.

Stamou A.I., Adams E.W. and Rodi W. (1989) Numerical modelling of flow and settling in primary rectangular clarifiers. J. Hydraul. Res., 27 (5), 665-682.

Standard Methods (1985) Standard methods for the examination of water and wastewater. 16th ed. AWWA, WEF, APHA, Washington D.C., USA. 
Stofkoper J.A. and Trentelman C.C.M. (1982) Richtlijen voor het dimensioneren van ronde nabezinktanks voor actiefslibinstallaties. $\mathrm{H}_{2} \mathrm{O}, 15$ (14), 334-354.

Takács I., Patry G.G. and Nolasco D. (1991) A dynamic model of the clarification thickening process. Wat. Res., 25 (10), 12631271.

Taylor C. and Hughes T.G (1981) Finite element programming of the NavierStokes equations. Pineridge Press, Swansea.

Turek S. (1998) Efficient solvers for incompressible flow problems: An algorithmic approach in view of computational aspects. Springer Verlag, Berlin.

Van Doormal J.P. and Raithby G.D. (1984) Enhancements of the SIMPLE method for prediction incompressible fluid flows. Numer. Heat Transfer Int. J. Comput. Methodol., 7, 147-163.

Van Kan J. (1986) A second order accurate pressure correction scheme for viscous incompressible flow. SIAM J. Sci. Stat. Comp., 7, 870-891.

Van Marle C. and Kranenburg C. (1994) Effects of gravity currents in circular secondary clarifiers. J. Environ. Eng., ASCE, 120 (4), 943-960.

Vesilind P.A. (1968) Design of prototype thickeners from batch settling tests. Wat. Sewage Wks, 115, 302-307.

Vitasovic Z.C., Zhou S., McCorquodale J.A. and Lingren K. (1997) Secondary clarifier analysis using data from the Clarifier Research Technical Committee protocol. Wat. Environ. Res., 69 (5), 999-1007.

Wahlberg E.J. and Keinath T.M. (1988) Development of settling flux curves using SVI. J. Wat. Pollut. Control Fed., 60 (12), 2005-2100.

Wahlberg E.J., Peterson M.A. and Flancher D.M. (1993) Field application of the $C R T C$ 's protocol for evaluating secondary clarifier performance: $A$ comparison of sludge removal mechanisms in circular clarifiers. Paper presented at Rocky Mountain Water Pollut. Control. Assoc. Annu. Meet., Albuquerque, N. Mex., USA.

Wahlberg E.J., Keinath, T.M. and Parker D.S. (1994) The influence of flocculation time in secondary clarification. Wat. Environ. Res., 66 (6), 779-786. 
Watts R.W., Svoronos S.A. and Koopman B. (1996) One dimensional modelling of secondary clarifiers using a concentration feed velocity dependent dispersion coefficient. Water Research, 30 (9), 2112-2124.

White M.J.D. (1975) Settling of activated sludge. Technical Report TR11, Water Research Centre, Stevenage, UK.

White M.J.D. (1976) Design and control of secondary settling tanks. Wat. Pollut. Control, 75 (4), 459-467.

Wilcox D.C. (1993) Turbulence modelling for CFD. DCW Industries, Inc., 5354 Palm Drive, La Cañada, California 91011.

Williams P.T. and Baker A.J. (1997) Numerical simulations of laminar flow over a $3 D$ backward-facing step. Int. J. Num. Methods Fluids, 24, 1159 $-1183$.

Wood W.L. (1984) A unified set of single-step algorithms. Part II: theory. Int. J. Num. Meth. Eng., 20, 2302-2309.

WPCF (1985) (Water Pollution Control Federation, now Water Environment Federation, WEF), Manual of Practice MOP-FD8 Clarifier Design, WEF, 601 Wythe St, Alexandria, VA 22314-1994, USA.

WRC Report No. 835/1/02 (2002) Hydrodynamic modelling of secondary settling tanks, Part 1: Application of a hydrodynamic model to full scale secondary settling tanks by G.A. Ekama and P. Marais, Part 2: Development of a finite element $2 D$ hydrodynamic model for secondary settling tanks by D. Kleine and B.D. Reddy. Water Research Commission (WRC), South Africa.

Zhou S. and McCorquodale J.A. (1992a) Modelling of rectandular settling tanks. J. Hydraul. Eng., ASCE, 118 (10), 1391-1405.

Zhou S. and McCorquodale J.A. (1992b) Mathematical modelling for a circular clarifier. Can. J. Civil Eng., CSCE, 19 (3), 365-374.

Zhou S., Pfeil, R., Strand E., Ji Z. and Vitasovic C. (1998) SettlerCAD (formerly Clarity) Release $1.1-A 2 D$ hydrodynamic model for secondary clarifiers. Reid Crowther Consulting, Seattle, WA. 
Zienkiewicz O.C. and Chung Y.K. (1965) Finite elements in the solution of field problems. The Engineer, 220, 507-510.

Zienkiewicz O.C, Wood W.L., Hine N.W. and Taylor R.L. (1984) A unified set of single-step algorithms. Part I: general formulation and applications. Int. J. Num. Meth. Eng., 20, 1529-1552.

Zienkiewicz O.C. and Taylor R.L. (1989) The finite element method. 4th ed., McGraw-Hill, Maidenhead, UK.

Zienkiewicz O.C. and Wu J. (1991) Incompressibility without tears! How to avoid restrictions of mixed formulations. Int. J. Num. Meth. Eng., 32, 1184-1203.

Zienkiewicz O.C. and Codina R. (1995) A general algorithm for compressible and incompressible flow - Part I: The split, characteristic-based scheme. Int. J. Num. Meth. Fluids, 10, 869-885.

Zienkiewicz O.C., Morgan K., Satya Sai B.V.K., Codina R. and Vazquez M. (1995) A general algorithm for compressible and incompressible flow - Part II: Tests on the explicit form. Int. J. Num. Meth. Fluids, 20, 887-913.

Zienkiewicz O.C., Nithiarasu P., Codina R., Vazquez M. and Ortiz P. (1999) The characteristic-based-split procedure: An efficient and accurate algorithm for fluid problems. Int. J. Num. Meth. Fluids, 31, 359-392.

Zienkiewicz O.C. and Taylor R.L. (2000) The finite element method. Vol. 3, Fluid dynamics, Butterworth-Heinemann, Oxford. 